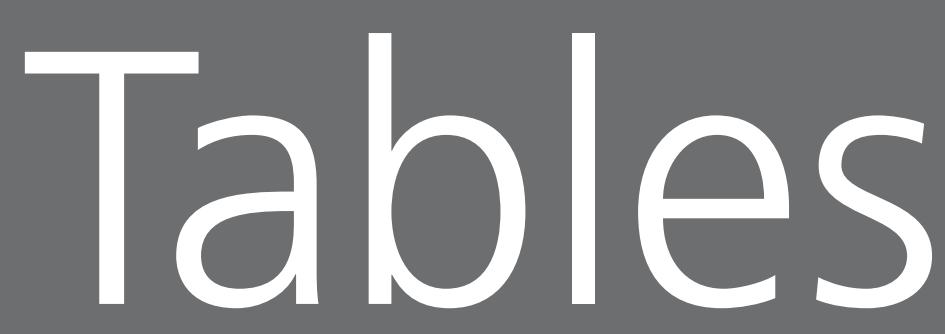

Table*

\title{
Population
}

International

Selected countries

Constituent countries of the United Kingdom

$1.4(6)$

National...

Government Office Regions

of England

..Constituent countries of

the United Kingdom

1.5 (7) Age, sex and legal marital status

...England and Wales

$1.6(5)$

Components of population change....

Constituent countries of

the United Kingdom

\section{Vital statistics}

Summary.

..Constituent countries of the United Kingdom

2.2 (new) Key demographic and health indicators

...Constituent countries of

\section{Live births}

the United Kingdom

Age of mother

England and Wales

Outside marriage: age of mother and type of registration .................England and Wales

Within marriage, within marriage to remarried women,

age of mother and birth order

England and Wales

\section{Conceptions and abortions}

4.1 (12) Age of women at conception.........................................................England and Wales (residents)

\section{Expectation of life}

5.1 (13) (In years) at birth and selected age ............................................................

\section{Deaths}

Age and sex the United Kingdom

\section{International migration}

Age and sex

United Kingdom

Country of last residence

United Kingdom

Citizenship

United Kingdom

\section{Internal migration}

8.1 (21) Movements within the United Kingdom............................................United Kingdom

\section{Marriage and divorce}

Remarriages: age, sex and previous marital status England and Wales

Divorces: age and sex

England and Wales

*Numbers in brackets indicate former table numbers in editions of Population Trends prior to spring 1999 (No 95). Former tables 16 and 17 (Deaths by selected causes, and Abortions) now appear in Health Statistics Quarterly.

Population Trends tables are also available in XLS or CSV formats via our website www.statistics.gov.uk

\section{Symbols}




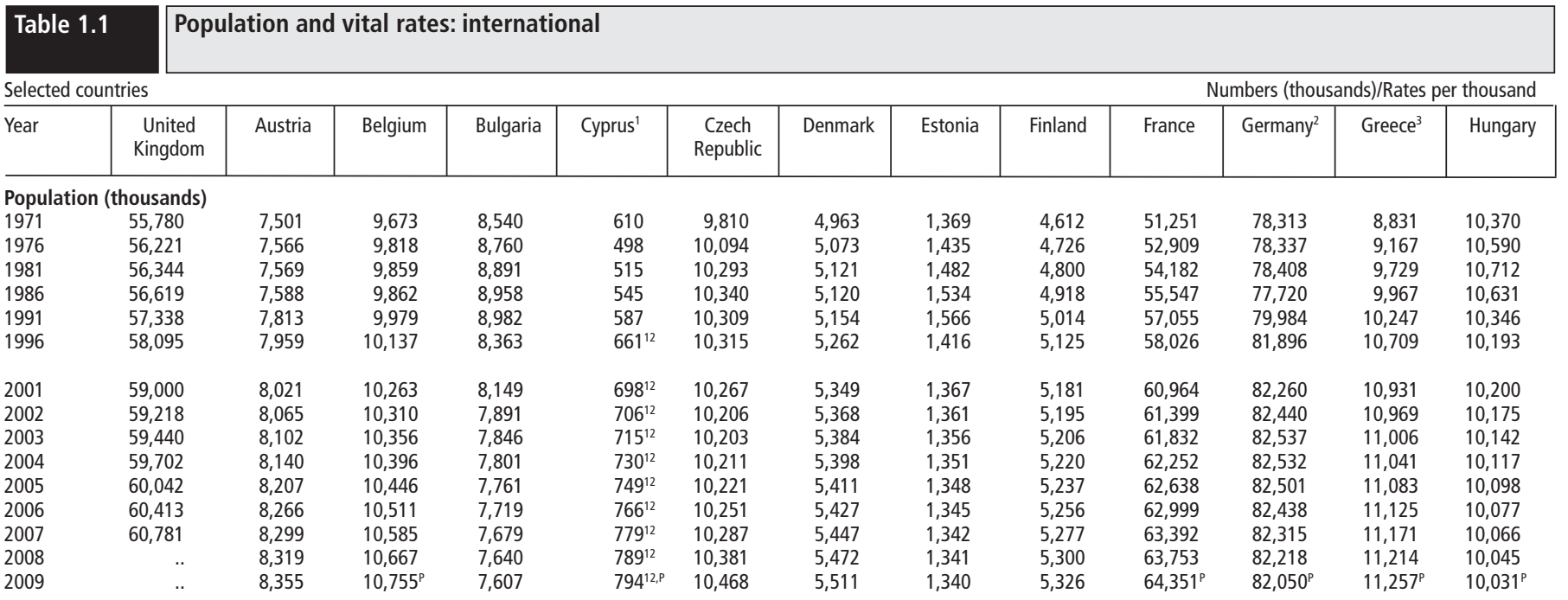

Population changes (per 1,000 per annum)

\begin{tabular}{|c|c|c|c|c|c|c|c|c|c|c|c|c|c|}
\hline \\
\hline 1971-76 & $1.6^{\circ}$ & 1.7 & 3.0 & 5.2 & -36.7 & 5.8 & 4.4 & 9.6 & 4.9 & 6.5 & 0.1 & 7.6 & 4.2 \\
\hline $1976-81$ & 0.4 & 0.1 & 0.8 & 3.0 & 6.8 & 3.9 & 1.9 & 6.6 & 3.1 & 4.8 & 0.2 & 12.3 & 2.3 \\
\hline 1981-86 & 1.0 & 0.5 & 0.1 & 1.5 & 11.7 & 0.9 & 0.0 & 7.0 & 4.9 & 5.0 & -1.8 & 4.9 & -1.5 \\
\hline 1986-91 & 2.5 & 5.9 & 2.4 & 0.5 & 15.4 & -0.6 & 1.3 & 4.2 & 3.9 & 5.4 & 5.8 & 5.6 & -5.4 \\
\hline $1991-96$ & 2.6 & 3.7 & 3.6 & -13.8 & 25.2 & 0.1 & 4.2 & -19.2 & 4.4 & 3.4 & 4.8 & 9.0 & -3.0 \\
\hline 1996-01 & 3.1 & 1.6 & 2.1 & -5.1 & 11.2 & -0.9 & 3.3 & -6.9 & 2.2 & 10.1 & 0.9 & 4.1 & 0.1 \\
\hline 2001-02 & 3.7 & 5.5 & 4.6 & -31.7 & 11.5 & -5.9 & 3.6 & -4.4 & 2.7 & 7.1 & 2.2 & 3.5 & -2.5 \\
\hline $2002-03$ & 3.7 & 4.6 & 4.5 & -5.7 & 12.7 & -0.3 & 3.0 & -3.7 & 2.1 & 7.1 & 1.2 & 3.4 & -3.2 \\
\hline 2003-04 & 4.4 & 4.7 & 3.9 & -5.7 & 21.0 & 0.8 & 2.6 & -3.7 & 2.7 & 6.8 & -0.1 & 3.2 & -2.5 \\
\hline 2004-05 & 5.7 & 8.2 & 4.8 & -5.1 & 26.0 & 1.0 & 2.4 & -2.2 & 3.3 & 6.2 & -0.4 & 3.8 & -1.9 \\
\hline $2005-06$ & 6.2 & 7.2 & 6.2 & -5.4 & 22.7 & 2.9 & 3.0 & -2.2 & 3.6 & 5.8 & -0.8 & 3.8 & -2.1 \\
\hline 2006-07 & 6.1 & 2.4 & 7.0 & -5.2 & 17.0 & 3.5 & 3.7 & -2.2 & 4.0 & 6.2 & -1.5 & 4.1 & -1.1 \\
\hline 2007-08 & .. & 2.4 & 7.7 & -5.1 & 12.8 & 9.1 & 4.6 & -0.7 & 4.4 & 5.7 & -1.2 & 3.8 & -2.1 \\
\hline 2008-09 & .. & 4.3 & 8.2 & -4.3 & 6.3 & 8.4 & 7.1 & -0.7 & 4.9 & 9.4 & -2.0 & 3.8 & -1.4 \\
\hline \multicolumn{14}{|c|}{ Live birth rate (per 1,000 population per annum) } \\
\hline $1971-75$ & 14.1 & 13.3 & 13.4 & 13.2 & 17.7 & 17.8 & 14.6 & 15.4 & 13.1 & 16.0 & 10.5 & 15.8 & 16.1 \\
\hline $1976-80$ & 12.5 & 11.5 & 12.5 & 15.1 & 19.0 & 17.1 & 12.0 & 15.0 & 13.6 & 14.1 & 10.5 & 15.6 & 15.8 \\
\hline 1981-85 & 12.9 & 12.0 & 12.0 & 13.7 & 20.2 & 13.5 & 10.2 & 15.6 & 13.4 & 14.2 & 10.7 & 13.3 & 12.3 \\
\hline $1986-90$ & 13.7 & 11.6 & 12.1 & 12.7 & 18.8 & 12.7 & 11.5 & 15.5 & 12.7 & 13.8 & 9.8 & 10.6 & 11.8 \\
\hline 1991-95 & 13.2 & 11.8 & 12.0 & 9.8 & 16.9 & 11.1 & 13.1 & 10.7 & 12.9 & 12.7 & 10.9 & 9.9 & 11.7 \\
\hline 1996-00 & 12.0 & 10.2 & 11.2 & 8.3 & 13.2 & 8.8 & 12.6 & 8.9 & 11.3 & 12.7 & 9.6 & 10.2 & 9.8 \\
\hline 2001 & 11.3 & 9.4 & 11.1 & 8.5 & 11.6 & 8.9 & 12.2 & 9.3 & 10.8 & 13.1 & 8.9 & 9.3 & 9.5 \\
\hline 2002 & 11.3 & 9.7 & 10.8 & 8.5 & 11.1 & 9.1 & 11.9 & 9.6 & 10.7 & 12.8 & 8.7 & 9.4 & 9.5 \\
\hline 2003 & 11.7 & 9.5 & 10.8 & 8.6 & 11.2 & 9.2 & 12.0 & 9.6 & 10.9 & 12.8 & 8.6 & 9.5 & 9.3 \\
\hline 2004 & 12.0 & 9.7 & 11.1 & 9.0 & 11.2 & 9.6 & 12.0 & 10.4 & 11.0 & 12.8 & 8.6 & 9.6 & 9.4 \\
\hline 2005 & 12.0 & 9.5 & 11.3 & 9.2 & 10.9 & 10.0 & 11.9 & 10.7 & 11.0 & 12.9 & 8.3 & 9.7 & 9.7 \\
\hline 2006 & 12.4 & 9.4 & 11.5 & 9.6 & 11.3 & 10.3 & 12.0 & 11.1 & 11.2 & 13.1 & 8.2 & 10.0 & 9.9 \\
\hline 2007 & 12.7 & 9.2 & 11.4 & 9.8 & 10.9 & 11.1 & 11.7 & 11.8 & 11.1 & 12.9 & 8.3 & 10.0 & 9.7 \\
\hline 2008 & $12.9^{\mathrm{P}}$ & 9.3 & 11.7 & 10.2 & 11.6 & 11.5 & 11.8 & 12.1 & 11.2 & 13.0 & 8.2 & 10.3 & 9.9 \\
\hline \multicolumn{14}{|c|}{ Death rate (per 1,000 population per annum) } \\
\hline $1971-75$ & 11.8 & 12.6 & 12.1 & 9.8 & 9.9 & 12.4 & 10.1 & 11.1 & 9.5 & 10.7 & 12.3 & 8.6 & 11.9 \\
\hline 1976-80 & 11.9 & 12.3 & 11.6 & 12.9 & 10.4 & 12.5 & 10.5 & 12.1 & 9.3 & 10.2 & 12.2 & 8.8 & 12.9 \\
\hline $1981-85$ & 11.7 & 12.0 & 11.4 & 11.3 & 10.0 & 12.8 & 11.1 & 12.3 & 9.3 & 10.1 & 12.0 & 9.0 & 13.7 \\
\hline $1986-90$ & 11.4 & 11.1 & 10.8 & 11.9 & 10.2 & 12.4 & 11.5 & 11.9 & 9.8 & 9.5 & 11.6 & 9.3 & 13.5 \\
\hline 1991-95 & 11.1 & 10.4 & 10.4 & 12.9 & 9.0 & 11.6 & 11.9 & 13.9 & 9.8 & 9.1 & 10.8 & 9.5 & 14.3 \\
\hline 1996-00 & 10.6 & 9.7 & 10.3 & 14.0 & 7.7 & 10.8 & 11.2 & 13.1 & 9.6 & 9.2 & 10.4 & 9.7 & 13.9 \\
\hline 2001 & 10.2 & 9.3 & 10.1 & 14.2 & 6.9 & 10.5 & 10.9 & 13.6 & 9.4 & 8.9 & 10.1 & 9.4 & 13.0 \\
\hline 2002 & 10.2 & 9.4 & 10.2 & 14.3 & 7.3 & 10.6 & 10.9 & 13.5 & 9.5 & 9.0 & 10.2 & 9.5 & 13.1 \\
\hline 2003 & 10.3 & 9.5 & 10.3 & 14.3 & 7.2 & 10.9 & 10.7 & 13.4 & 9.4 & 9.2 & 10.3 & 9.6 & 13.4 \\
\hline 2004 & 9.7 & 9.1 & 9.8 & 14.2 & 7.1 & 10.5 & 10.3 & 13.2 & 9.1 & 8.4 & 10.0 & 9.5 & 13.1 \\
\hline 2005 & 9.7 & 9.1 & 9.9 & 14.7 & 7.2 & 10.6 & 10.2 & 12.9 & 9.1 & 8.6 & 10.1 & 9.5 & 13.5 \\
\hline 2006 & 9.4 & 9.0 & 9.6 & 14.8 & 6.7 & 10.2 & 10.2 & 12.9 & 9.1 & 8.5 & 9.9 & 9.5 & 13.1 \\
\hline 2007 & 9.4 & 9.0 & 9.5 & 14.8 & 6.9 & 10.1 & 10.2 & 13.0 & 9.3 & 8.4 & 10.0 & 9.8 & 13.0 \\
\hline 2008 & $9.4^{\mathrm{p}}$ & 9.0 & 9.5 & 14.5 & 6.4 & 10.1 & 9.9 & 12.5 & 9.2 & 8.4 & 10.3 & 9.3 & 13.0 \\
\hline
\end{tabular}

Note: Estimated population (as at 1 January) live birth and death rates up to the latest available data, as given in the United Nations Monthly Bulletin of Statistics, the United Nations

Demographic Yearbook, and the Eurostat website (July 2009).

Birth and death rates for 2008 have been calculated using 2006-based population projection for 2008

These will be revised later in 2009

1 Republic of Cyprus - Data refer to Government controlled areas.

2 Including the former GDR throughout.

3 Greece - Mid-year population excludes armed forces stationed outside the country but includes alien forces stationed in the area.

4 Malta - including work and resident permit holders and foreigners residing in Malta.

5 Poland-excluding civilian aliens within the country but including civilian nationals temporarily outside the country.

6 Portugal - including the Azores and Madeira Islands.

7 Spain - including the Balearic and Canary Islands.

8 For 1971 the European Union consisted of the 6 original member countries. This has since been expanded to include: 9 countries (1976-EU15); 10 countries (2004-EU25); 2 countries (2007-

expanded to include: 9 countries (1976-EU15);
EU27). In this table, all totals include the EU27.
Including the Indian held part of Jammu and Kashmir, the final status of which has not yet been determined.

10 Japan - excluding diplomatic personnel outside the country and foreign military and civilian personnel and their dependants stationed in the area.

11 USA - excluding armed forces overseas and civilian citizens absent from the country for extended periods.

12 Indicates population estimates of uncertain reliability.

13 Data refers to 15 April.

14 Mid-year estimates have been adjusted for under-enumeration.

15 For statistical purposes the data for China do not include those for the Hong Kong SAR, Macao SAR and Taiwan province of China. Data for the period 1996 to 2000 have been adjusted on the basis of the Population Census of 2000. Data from 2001 to 2007 have been estimated on the basis of the annual national sample surveys of Population Changes.

16 Rate is for 1990-1995.

$s$ Eurostat estimate

$\mathrm{p}$ provisional 


\begin{tabular}{|c|c|c|c|c|c|c|c|c|c|c|c|c|c|c|}
\hline \multicolumn{2}{|c|}{$\begin{array}{l}\text { Table } 1.1 \\
\text { continued }\end{array}$} & \multicolumn{13}{|c|}{ Population and vital rates: international } \\
\hline \multicolumn{11}{|c|}{ Selected countries } & \multicolumn{4}{|c|}{ Numbers (thousands)/Rates per thousand } \\
\hline$\overline{\text { Year }}$ & $\begin{array}{c}\text { Irish } \\
\text { Republic }\end{array}$ & Italy & Latvia & \begin{tabular}{|l|} 
Lithuania \\
\end{tabular} & $\begin{array}{l}\text { Luxem- } \\
\text { bourg }\end{array}$ & Malta $^{4}$ & $\begin{array}{l}\text { Nether- } \\
\text { lands }\end{array}$ & Poland $^{5}$ & Portugal $^{6}$ & Romania & Slovakia & Slovenia & Spain 7 & Sweden \\
\hline \multicolumn{15}{|c|}{ Population (thousands) } \\
\hline 1971 & 2,992 & 54,073 & 2,366 & 3,160 & 342 & 330 & 13,194 & 32,800 & 8,644 & 20,470 & 4,540 & 1,732 & 34,216 & 8,098 \\
\hline 1976 & 3,238 & 55,718 & 2,465 & 3,315 & 361 & 330 & 13,774 & 34,360 & 9,356 & 21,450 & 4,764 & 1,809 & 36,118 & 8,222 \\
\hline 1981 & 3,443 & 56,502 & 2,515 & 3,422 & 365 & 322 & 14,247 & 35,902 & 9,851 & 22,353 & 4,996 & 1,910 & 37,741 & 8,320 \\
\hline 1986 & 3,543 & 56,596 & 2,588 & 3,560 & 368 & 344 & 14,572 & 37,456 & 10,011 & 22,823 & 5,179 & 1,975 & 38,536 & 8,370 \\
\hline 1991 & 3,526 & 56,751 & 2,662 & 3,742 & 387 & 358 & 15,070 & 38,245 & 9,871 & 23,185 & 5,283 & 2,002 & 38,920 & 8,617 \\
\hline 1996 & $3,626^{13}$ & 56,860 & 2,457 & 3,602 & 414 & 380 & 15,530 & 38,618 & 10,058 & 22,608 & 5,374 & 1,991 & 39,479 & 8,841 \\
\hline 2001 & $3,833^{13}$ & 56,960 & 2,364 & 3,487 & 439 & 391 & 15,987 & 38,254 & 10,257 & 22,430 & 5,379 & 1,990 & 40,477 & 8,883 \\
\hline 2002 & $3,900^{13}$ & 56,994 & 2,346 & 3,476 & 444 & 395 & 16,105 & 38,242 & 10,329 & 21,833 & 5,379 & 1,994 & 40,964 & 8,909 \\
\hline 2003 & $3,964^{13}$ & 57,321 & 2,331 & 3,463 & 448 & 397 & 16,193 & 38,219 & 10,407 & 21,773 & 5,379 & 1,995 & 41,664 & 8,941 \\
\hline 2004 & $4,028^{13}$ & 57,888 & 2,319 & 3,446 & 455 & 400 & 16,258 & 38,191 & 10,475 & 21,711 & 5,380 & 1,996 & 42,345 & 8,976 \\
\hline 2005 & $4,109^{13}$ & 58,462 & 2,306 & $\begin{array}{l}3,440 \\
3,425\end{array}$ & 461 & 403 & 16,306 & 38,174 & 10,529 & 21,659 & 5,385 & $\begin{array}{l}1,998 \\
1,989\end{array}$ & 43,038 & 9,011 \\
\hline 2006 & $4,209^{13}$ & 58,752 & 2,295 & 3,403 & 469 & 405 & 16,334 & 38,157 & 10,570 & 21,610 & 5,389 & 2,003 & 43,758 & 9,048 \\
\hline 2007 & $4,313^{13}$ & 59,131 & 2,281 & 3,385 & 476 & 408 & 16,358 & 38,125 & 10,599 & 21,565 & 5,394 & 2,010 & 44,475 & 9,113 \\
\hline 2008 & $4,401^{13}$ & 59,619 & 2,271 & 3,366 & 484 & 410 & 16,405 & 38,116 & 10,618 & 21,529 & 5,401 & 2,010 & 45,283 & 9,183 \\
\hline 2009 & $4,466^{13, p}$ & $60,053^{\mathrm{P}}$ & 2,261 & 3,350 & 494 & 414 & $16,487^{p}$ & 38,136 & $10,627^{\mathrm{p}}$ & 21,499 & 5,412 & 2,032 & 45,828 & 9,256 \\
\hline \multicolumn{15}{|c|}{ Population changes (per 1,000 per annum) } \\
\hline $1971-76$ & 16.4 & 6.1 & 8.4 & 9.8 & 10.7 & 0.0 & 8.8 & 9.5 & 16.5 & 9.6 & 9.9 & 8.9 & 11.1 & 3.1 \\
\hline $1976-81$ & 12.7 & 2.8 & 4.1 & 6.5 & 2.5 & -4.8 & 6.9 & 9.0 & 10.6 & 8.4 & 9.7 & 11.2 & 9.0 & 2.4 \\
\hline $1981-86$ & 5.8 & 0.3 & 5.8 & 8.1 & 1.8 & 13.7 & 4.6 & 8.7 & 3.2 & 4.2 & 7.3 & 6.8 & 4.2 & 1.2 \\
\hline $1986-91$ & $\begin{array}{l}-1.0 \\
-1.0\end{array}$ & 0.5 & 5.7 & 10.2 & 10.2 & 8.1 & $\begin{array}{l}6.8 \\
6.8\end{array}$ & 4.2 & $\begin{array}{l}-2.2 \\
-2.8\end{array}$ & 3.2 & 4.0 & 2.7 & 2.0 & 5.9 \\
\hline $1991-96$ & 5.7 & 0.4 & -15.4 & -7.5 & 13.9 & 12.3 & 6.1 & 2.0 & 3.8 & -5.0 & 3.4 & -1.1 & 2.9 & 5.2 \\
\hline $1996-01$ & 11.4 & 0.4 & -7.6 & -6.4 & 12.1 & 5.8 & 5.9 & -1.9 & 4.0 & -1.6 & 0.2 & -0.1 & 5.1 & 1.0 \\
\hline 2001-02 & 17.5 & 0.6 & -7.6 & -3.2 & 11.4 & 10.2 & 7.4 & -0.3 & 7.0 & -26.6 & 0.0 & 2.0 & 12.0 & 2.9 \\
\hline 2002-03 & 16.4 & 5.7 & -6.4 & -3.7 & 9.0 & 5.1 & 5.5 & -0.6 & 7.6 & -2.7 & 0.0 & 0.5 & 17.1 & 3.6 \\
\hline 2003-04 & 16.1 & 9.9 & -5.1 & -4.9 & 15.6 & 7.6 & 4.0 & -0.7 & 6.5 & -2.8 & 0.2 & 0.5 & 16.3 & 3.9 \\
\hline 2004-05 & 20.1 & 9.9 & -5.6 & -6.1 & 13.2 & 7.5 & 3.0 & -0.4 & 5.2 & -2.4 & 0.9 & 1.0 & 16.4 & 3.9 \\
\hline 2005-06 & 24.3 & 5.0 & -4.8 & -6.4 & 17.4 & 5.0 & 1.7 & -0.4 & 3.9 & -2.3 & 0.7 & 2.5 & 16.7 & 4.1 \\
\hline $2006-07$ & 24.7 & 6.5 & -6.1 & -5.3 & 14.9 & 7.4 & 1.5 & -0.8 & 2.7 & -2.1 & 0.9 & 3.5 & 16.4 & 7.2 \\
\hline 2007-08 & 20.4 & 8.3 & -4.4 & -5.6 & 16.8 & 4.9 & 2.9 & -0.2 & 1.8 & -1.7 & 1.3 & 0.0 & 18.2 & 7.7 \\
\hline $2008-09$ & 14.8 & 7.3 & -4.4 & -4.8 & 20.7 & 9.8 & 5.0 & 0.5 & 0.8 & -1.4 & 2.0 & 10.9 & 12.0 & 7.9 \\
\hline \multicolumn{15}{|c|}{ Live birth rate (per 1,000 population per annum) } \\
\hline $1971-75$ & 22.2 & 16.0 & 14.4 & 16.4 & 11.6 & 17.5 & 14.9 & 17.9 & 20.3 & 19.3 & 19.7 & 16.4 & 19.2 & 13.5 \\
\hline $1976-80$ & 21.3 & 12.6 & 13.9 & 15.4 & 11.2 & 17.0 & 12.6 & 19.3 & 17.9 & 18.9 & 20.3 & 16.3 & 17.1 & 11.6 \\
\hline $1981-85$ & 19.2 & 10.6 & 15.2 & 16.0 & 11.6 & 15.3 & 12.2 & 19.0 & 14.5 & 15.6 & 18.0 & 14.2 & 12.8 & 11.3 \\
\hline $1986-90$ & 15.8 & 9.8 & 15.3 & 15.8 & 12.2 & 16.0 & 12.8 & 15.5 & 11.9 & 15.8 & 15.8 & 12.3 & 10.8 & 13.2 \\
\hline $1991-95$ & 14.0 & 9.6 & 10.8 & 13.1 & 13.3 & 14.0 & 12.8 & 12.9 & 11.4 & 11.1 & 13.3 & 10.0 & 9.8 & 13.3 \\
\hline $1996-00$ & 14.2 & 9.2 & 8.0 & 10.4 & 13.1 & 12.0 & 12.6 & 10.4 & 11.3 & 10.4 & 10.7 & 9.1 & 9.5 & 10.2 \\
\hline 2001 & 15.0 & 9.4 & 8.4 & 9.1 & 12.4 & 10.1 & 12.6 & 9.6 & 11.0 & 10.1 & 9.5 & 8.8 & 10.0 & 10.3 \\
\hline 2002 & 15.4 & 9.4 & 8.6 & 8.7 & 12.0 & $\begin{array}{l}9.1 \\
9.9\end{array}$ & 12.5 & 9.3 & 11.0 & 9.7 & 9.5 & 8.8 & 10.2 & 10.7 \\
\hline 2003 & 15.4 & 9.4 & 9.0 & 8.9 & 11.7 & 10.2 & 12.3 & 9.2 & 10.8 & 9.8 & 9.6 & 8.7 & 10.5 & 11.1 \\
\hline 2004 & 15.2 & 9.7 & 8.8 & 8.9 & 11.9 & 9.7 & 11.9 & 9.3 & 10.4 & 10.0 & 10.0 & 9.0 & 10.6 & 11.2 \\
\hline 2005 & 14.7 & 9.5 & 9.3 & 9.0 & 11.5 & 9.6 & 11.5 & 9.5 & 10.4 & 10.2 & 10.1 & 9.1 & 10.7 & 11.2 \\
\hline 2006 & 15.1 & 9.5 & 9.7 & 9.2 & 11.7 & 9.6 & 11.3 & 9.8 & 10.0 & 10.2 & 10.0 & 9.4 & 10.9 & 11.7 \\
\hline 2007 & 16.2 & 9.5 & 10.2 & 9.6 & 11.4 & 9.5 & 11.0 & $\begin{array}{r}10.2 \\
10.2\end{array}$ & 9.7 & 10.0 & 10.1 & $\begin{array}{l}9.4 \\
9.8\end{array}$ & 11.0 & 11.7 \\
\hline 2008 & 16.9 & 9.6 & 10.6 & 10.4 & 11.5 & 10.0 & 11.2 & 10.9 & 9.8 & 10.3 & 10.6 & 10.5 & 11.4 & 11.9 \\
\hline \multicolumn{15}{|c|}{ Death rate (per 1.000 population per annum) } \\
\hline $1971-75$ & 11.0 & 9.8 & 11.6 & 9.0 & 12.2 & 9.0 & 8.3 & 8.4 & 11.0 & 9.4 & 9.4 & 10.0 & 8.5 & 10.5 \\
\hline $1976-80$ & 10.2 & 9.7 & 12.6 & 10.1 & 11.5 & 9.0 & 8.1 & 9.2 & 10.1 & 9.8 & 9.8 & 9.8 & 8.0 & 10.9 \\
\hline $1981-85$ & 9.4 & 9.5 & 12.8 & 10.6 & 11.2 & 8.2 & 8.3 & 9.6 & 9.6 & 10.3 & 10.1 & 10.3 & 7.7 & 11.0 \\
\hline $1986-90$ & 9.1 & 9.4 & 12.4 & 10.3 & 10.5 & 7.4 & 8.5 & 10.0 & 9.6 & 10.8 & 10.1 & 9.6 & 8.2 & 11.1 \\
\hline $1991-95$ & 8.8 & 9.7 & 14.8 & 12.0 & 9.8 & 7.6 & 8.8 & 10.2 & 10.4 & 11.5 & 9.9 & 9.7 & 8.7 & 10.9 \\
\hline $1996-00$ & 8.5 & 9.8 & 13.9 & 11.5 & 9.0 & 7.7 & 8.8 & 9.8 & 10.5 & 12.0 & 9.7 & 9.5 & 9.1 & 10.6 \\
\hline 2001 & 7.9 & 9.6 & 14.0 & 11.6 & 8.4 & 7.6 & 8.7 & 9.5 & 10.2 & 11.6 & 9.7 & 9.3 & 8.9 & 10.5 \\
\hline 2002 & 7.5 & 9.8 & 13.9 & 11.8 & 8.4 & 7.8 & 8.8 & 9.4 & 10.2 & 12.4 & 9.6 & 9.4 & 8.9 & 10.6 \\
\hline 2003 & 7.2 & 10.2 & 13.9 & 11.9 & $\begin{array}{l}9.4 \\
9.0\end{array}$ & $\begin{array}{l}1.0 \\
7.9\end{array}$ & $\begin{array}{l}8.0 \\
8.7\end{array}$ & $\begin{array}{l}9.4 \\
9.6\end{array}$ & 10.4 & 12.3 & 9.7 & $\begin{array}{l}9.4 \\
9.7\end{array}$ & 9.2 & 10.4 \\
\hline 2004 & 7.0 & 9.4 & 13.9 & 12.0 & 7.6 & 7.5 & 8.4 & 9.5 & 9.7 & 11.9 & 9.6 & 9.3 & 8.7 & 10.1 \\
\hline 2005 & 6.6 & 9.7 & 14.2 & 12.8 & 8.0 & 7.8 & 8.4 & 9.7 & 10.2 & 12.1 & 9.9 & 9.4 & 8.9 & 10.2 \\
\hline 2006 & 6.5 & 9.5 & 14.5 & 13.2 & 8.0 & 7.9 & 8.3 & 9.7 & 9.7 & 11.9 & 9.9 & 9.1 & 8.4 & 10.0 \\
\hline 2007 & 6.6 & 9.6 & 14.5 & 13.5 & 8.1 & 7.6 & 8.1 & 9.9 & 9.8 & 11.7 & 9.9 & 9.2 & $\begin{array}{l}8.4 \\
8.6\end{array}$ & 10.0 \\
\hline 2008 & 6.1 & 9.8 & 13.8 & 13.1 & 7.4 & 7.7 & 8.2 & 10.0 & 9.7 & 11.8 & 9.8 & 9.1 & 8.7 & 9.9 \\
\hline
\end{tabular}

See notes on first page of table. 
Table 1.1

continued

Population and vital rates: international

Selected countries

Numbers (thousands)/Rates per thousand

\begin{tabular}{|c|c|c|c|c|c|c|c|c|c|c|}
\hline Year & $\mathrm{EU}^{8}$ & $\begin{array}{l}\text { Russian } \\
\text { Federation }\end{array}$ & Australia & Canada & $\begin{array}{c}\text { New } \\
\text { Zealand }\end{array}$ & China & India ${ }^{9}$ & Japan $^{10}$ & USA $^{11}$ & Year \\
\hline \multicolumn{11}{|c|}{ Population (thousands) } \\
\hline 1971 & 438,728 & 130,934 & 13,067 & 22,026 & 2,899 & $852,290^{15}$ & 551,311 & 105,145 & 207,661 & 1971 \\
\hline 1976 & 450,468 & 135,027 & 14,033 & 23,517 & 3,163 & $937,170^{15}$ & 617,248 & 113,094 & 218,035 & 1976 \\
\hline 1981 & 459,807 & 139,225 & 14,923 & 24,900 & 3,195 & $1,008,460^{15}$ & 675,185 & 117,902 & 229,958 & 1981 \\
\hline 1986 & 465,336 & 144,154 & 16,018 & 26,204 & 3,317 & $1,086,733^{15}$ & 767,199 & 121,672 & 240,680 & 1986 \\
\hline 1991 & 473,094 & 148,245 & 17,284 & 28,031 & 3,477 & $1,170,100^{15}$ & 851,897 & 123,964 & 252,639 & 1991 \\
\hline 1996 & 478,084 & 148,160 & $18,311^{14}$ & $29,611^{14}$ & 3,732 & $1,217,550^{15}$ & $942,157^{12}$ & 125,757 & 269,394 & 1996 \\
\hline 2001 & 483,782 & 145,976 & $19,413^{14}$ & $31,021^{14}$ & 3,880 & $1,271,850^{12,15}$ & $1,035,066^{12}$ & 127,150 & 285,108 & 2001 \\
\hline 2002 & 484,614 & 145,306 & $19,650^{14}$ & $31,373^{14}$ & 3,939 & $1,280,400^{12,15}$ & $1,051,260^{12}$ & 127,450 & 287,985 & 2002 \\
\hline 2003 & 486,617 & 144,566 & $19,990^{14}$ & $31,669^{14}$ & 4,009 & $1,288,400^{12,15}$ & $1,068,070^{12}$ & 127,720 & 290,850 & 2003 \\
\hline 2004 & 488,757 & 143,821 & $20,140^{14}$ & $31,974^{14}$ & 4,061 & $1,296,075^{12,15}$ & $1,085,600^{12}$ & 127,760 & 293,623 & 2004 \\
\hline 2005 & 491,024 & 143,110 & $20,409^{14}$ & $32,312^{14}$ & 4,099 & $1,303,720^{12,15}$ & $1,101,320^{12}$ & 127,773 & 296,410 & 2005 \\
\hline 2006 & 492,975 & 142,490 & $20,700^{14}$ & $32,650^{14}$ & 4,180 & $1,311,020^{12,15}$ & $1,117,730^{12}$ & 127,760 & 299,400 & 2006 \\
\hline 2007 & 495,090 & 142,110 & $21,070^{14}$ & $32,980^{14, P}$ & 4,230 & $1,324,660^{12,15}$ & $1,134,020^{12}$ & 127,770 & 301,620 & 2007 \\
\hline 2008 & $497,660^{P}$ & .. & $21,430^{14, P}$ & .. & 4,270 & .. & $1,150,000^{12}$ & 127,700 & .. & 2008 \\
\hline 2009 & $499,795^{P}$ & .. & .. & .. & .. & .. & .. & .. & .. & 2009 \\
\hline
\end{tabular}

Population changes (per 1,000 per annum)

$\begin{array}{lcc}\text { Population changes (per } 1,000 \text { per annum) } \\ \text { 1971-76 } & 5.4 & \\ 1976-81 & 4.1 & \\ 1981-86 & 2.4 & \\ 1986-91 & 3.3 & \\ 1991-96 & 2.1 & -1.7 \\ 1996-01 & 2.4 & -2.9 \\ 2001-02 & & \\ 2002-03 & 1.7 & -4.6 \\ 2003-04 & 4.1 & -5.1 \\ 2004-05 & 4.4 & -5 . \\ 2005-06 & 4.6 & -4.9 \\ 2006-07 & 4.0 & -4.3 \\ 2007-2008 & 4.3 & -2.7 \\ 2008-09 & 5.2 & \end{array}$

$\begin{array}{rrrr}6.3 & 14.8 & 13.5 & 18.2 \\ 6.2 & 12.7 & 11.8 & 2.0 \\ 7.1 & 14.7 & 10.5 & 7.6 \\ 5.7 & 15.8 & 13.9 & 9.6 \\ -1.7 & 11.9 & 11.3 & 14.7 \\ -2.9 & 12.0 & 9.5 & 7.9 \\ -4.6 & 12.2 & 11.3 & 15.2 \\ -5.1 & 17.3 & 9.4 & 17.8 \\ -5.2 & 7.5 & 9.6 & 13.0 \\ -4.9 & 13.4 & 10.6 & 9.4 \\ -4.3 & 14.3 & 10.5 & 19.8 \\ -2.7 & 17.9 & 10.1 & 12.0 \\ . . & 17.1 & . . & 9.5 \\ . . & . . & . . & \end{array}$

$\begin{array}{rrr}18.2 & 19.9 & 23.9 \\ 2.0 & 15.2 & 18.8 \\ 7.6 & 15.5 & 27.3 \\ 9.6 & 15.3 & 22.1 \\ 14.7 & 10.3 & 21.1 \\ 7.9 & 8.9 & 19.7\end{array}$

23.9
18.8
27.3
22.1
21.1
19.7

$\begin{array}{rrr}15.1 & 10.0 & 1971-76 \\ 8.5 & 10.9 & 1976-81 \\ 6.4 & 9.3 & 1981-86 \\ 3.8 & 9.9 & 1986-91 \\ 2.9 & 12.1 & 1991-96 \\ 2.2 & 11.7 & 1996-01 \\ & & \\ 2.4 & 10.1 & 2001-02 \\ 2.1 & 9.9 & 2002-03 \\ 0.3 & 9.5 & 2003-04 \\ 0.1 & 9.5 & 2004-05 \\ -0.1 & 10.1 & 2005-06 \\ 0.1 & 7.4 & 2006-07 \\ 0.5 & . . & 2007-08 \\ . . & . . & 2008-09\end{array}$

Live birth rate (per 1,000 population per annum)

\section{1-75}

$1976-80$

1981-85

1986-90

1991-95

1996-00

$\begin{array}{lll}2001 & 10.4 & \\ 2002 & 10.3 & \\ 2003 & 10.3 & 10.2 \\ 2004 & 10.4 & 10.4 \\ 2005 & 10.4 & 10.2 \\ 2006 & 10.6 & 10.4 \\ 2007 & 10.6 & 11.3 \\ 2008 & 10.9 & \end{array}$

Death rate (per 1,000 population per annum)

\begin{tabular}{|c|c|c|c|c|c|c|c|c|c|c|}
\hline $1971-75$ & .. & .. & 8.2 & 7.4 & 8.4 & 7.3 & 15.5 & 6.4 & 9.1 & $1971-75$ \\
\hline $1976-80$ & .. & .. & 7.6 & 7.2 & 8.2 & 6.6 & 13.8 & 6.1 & 8.7 & $1976-80$ \\
\hline $1981-85$ & .. & .. & 7.3 & 7.0 & 8.1 & 6.7 & .. & 6.1 & 8.6 & $1981-85$ \\
\hline $1986-90$ & .. & .. & 7.2 & 7.3 & 8.2 & .. & .. & 6.4 & 8.7 & $1986-90$ \\
\hline 1991-95 & 10.4 & 13.7 & 7.0 & 7.8 & 7.8 & .. & .. & 7.0 & 8.7 & $1991-95$ \\
\hline $1996-00$ & 10.2 & 14.3 & 6.9 & 7.2 & 7.2 & .. & .. & 7.4 & 8.5 & 1996-00 \\
\hline 2001 & 9.9 & 15.4 & 6.6 & 7.1 & 7.2 & $6.4^{15}$ & 8.4 & 7.6 & 8.5 & 2001 \\
\hline 2002 & 9.9 & 16.1 & 6.8 & 7.1 & 7.1 & $6.4^{15}$ & 8.1 & 7.7 & 8.5 & 2002 \\
\hline 2003 & 10.1 & 16.4 & 6.7 & 7.1 & 7.0 & $6.4^{15}$ & 8.0 & 7.9 & 8.4 & 2003 \\
\hline 2004 & 9.7 & 16.0 & 6.6 & 7.1 & 7.0 & $6.4^{15}$ & 7.5 & 8.1 & 8.2 & 2004 \\
\hline 2005 & 9.8 & 16.1 & 6.4 & 7.1 & 6.6 & $6.5^{15}$ & 7.6 & 8.5 & 8.3 & 2005 \\
\hline 2006 & .. & 15.2 & 6.5 & 7.1 & 6.7 & $6.8^{15}$ & 7.5 & 8.5 & 8.1 & 2006 \\
\hline 2007 & .. & 14.7 & 6.6 & .. & 6.8 & $6.9^{15}$ & 7.4 & 8.8 & .. & 2007 \\
\hline 2008 & 9.7 & .. & .. & .. & 6.8 & .. & .. & .. & .. & 2008 \\
\hline
\end{tabular}

See notes on first page of table.

$\begin{array}{rr}. . & \\ . . & \\ . . & \\ \ddot{1} & \\ 11.4 & 10.2 \\ 10.6 & 8.6 \\ 10.4 & \\ 10.3 & \\ 10.3 & \\ 10.4 & 10.2 \\ 10.4 & 10.4 \\ 10.6 & 10.2 \\ 10.6 & 10.4 \\ 10.9 & 11.3 \\ & \end{array}$

15.9

15.5

15.1

14.8

13.6
11.4

10.8

10.5

10.6

10.5

10.7

$\begin{array}{ccr}20.4 & 27.2 & 35.6 \\ 16.8 & 18.6 & 33.4 \\ 15.8 & 19.2 & . . \\ 17.1 & . . & . . \\ 16.9 & 18.5^{16} & . . \\ 14.9 & . . & . . \\ & & \\ 14.4 & 13.4^{15} & 25.4 \\ 13.7 & 12.9^{15} & 25.0 \\ 14.0 & 12.4^{15} & 24.8 \\ 14.3 & 12.3^{15} & 24.1 \\ 14.1 & 12.4^{15} & 23.8 \\ 14.1 & 12.1^{15} & 23.5 \\ 15.2 & 12.0^{15} & 23.1\end{array}$

$\begin{array}{rr}15.3 & 1971-75 \\ 15.2 & 1976-80 \\ 15.7 & 1981-85 \\ 16.0 & 1986-90 \\ 13.1 & 1991-95 \\ 14.3 & 1996-00 \\ 14.1 & \\ 14.0 & 2001 \\ 14.1 & 2002 \\ 14.0 & 2003 \\ 14.0 & 2004 \\ 14.2 & 2005 \\ . . & 2006 \\ . . & 2007 \\ & 2008\end{array}$




\section{Table 1.2}

Mid Year Population: national

\begin{tabular}{|c|c|c|c|c|c|c|c|}
\hline \multicolumn{5}{|c|}{ Constituent countries of the United Kingdom } & \multicolumn{3}{|c|}{ Numbers (thousands) and percentage age distribution } \\
\hline Mid-year & $\begin{array}{l}\text { United } \\
\text { Kingdom }\end{array}$ & $\begin{array}{l}\text { Great } \\
\text { Britain }\end{array}$ & $\begin{array}{l}\text { England } \\
\text { and Wales }\end{array}$ & England & Wales & Scotland & $\begin{array}{l}\text { Northern } \\
\text { Ireland }\end{array}$ \\
\hline \multicolumn{8}{|l|}{ Estimates } \\
\hline 1971 & 55,928 & 54,388 & 49,152 & 46,412 & 2,740 & 5,236 & 1,540 \\
\hline 1976 & 56,216 & 54,693 & 49,459 & 46,660 & 2,799 & 5,233 & 1,524 \\
\hline 1981 & 56,357 & 54,815 & 49,634 & 46,821 & 2,813 & 5,180 & 1,543 \\
\hline 1986 & 56,684 & 55,110 & 49,999 & 47,188 & 2,811 & 5,112 & 1,574 \\
\hline 1991 & 57,439 & 55,831 & 50,748 & 47,875 & 2,873 & 5,083 & 1,607 \\
\hline 1993 & 57,714 & 56,078 & 50,986 & 48,102 & 2,884 & 5,092 & 1,636 \\
\hline 1994 & 57,862 & 56,218 & 51,116 & 48,229 & 2,887 & 5,102 & 1,644 \\
\hline 1995 & 58,025 & 56,376 & 51,272 & 48,383 & 2,889 & 5,104 & 1,649 \\
\hline 1996 & 58,164 & 56,503 & 51,410 & 48,519 & 2,891 & 5,092 & 1,662 \\
\hline 1997 & 58,314 & 56,643 & 51,560 & 48,665 & 2,895 & 5,083 & 1,671 \\
\hline 1998 & 58,475 & 56,797 & 51,720 & 48,821 & 2,900 & 5,077 & 1,678 \\
\hline 1999 & 58,684 & 57,005 & 51,933 & 49,033 & 2,901 & 5,072 & 1,679 \\
\hline 2000 & 58,886 & 57,203 & 52,140 & 49,233 & 2,907 & 5,063 & 1,683 \\
\hline 2001 & 59,113 & 57,424 & 52,360 & 49,450 & 2,910 & 5,064 & 1,689 \\
\hline 2002 & 59,323 & 57,627 & 52,572 & 49,652 & 2,920 & 5,055 & 1,697 \\
\hline 2003 & 59,557 & 57,855 & 52,797 & 49,866 & 2,931 & 5,057 & 1,703 \\
\hline 2004 & 59,846 & 58,136 & 53,057 & 50,111 & 2,946 & 5,078 & 1,710 \\
\hline 2005 & 60,238 & 58,514 & 53,419 & 50,466 & 2,954 & 5,095 & 1,724 \\
\hline 2006 & 60,587 & 58,846 & 53,729 & 50,763 & 2,966 & 5,117 & 1,742 \\
\hline 2007 & 60,975 & 59,216 & 54,072 & 51,092 & 2,980 & 5,144 & 1,759 \\
\hline 2007 by age $g$ & & & & & & & \\
\hline $0-4$ & 5.9 & 5.9 & 5.9 & 5.9 & 5.5 & 5.3 & 6.6 \\
\hline 5-15 & 13.0 & 12.9 & 13.0 & 13.0 & 13.2 & 12.5 & 15.1 \\
\hline $16-44$ & 40.1 & 40.1 & 40.2 & 40.3 & 37.4 & 39.3 & 41.2 \\
\hline $45-64 \mathrm{M} / 59 \mathrm{~F}$ & 22.0 & 22.1 & 21.9 & 21.9 & 22.9 & 23.4 & 20.7 \\
\hline $65 \mathrm{M} / 60 \mathrm{~F}-74$ & 11.2 & 11.3 & 11.2 & 11.1 & 12.5 & 11.9 & 10.1 \\
\hline 75 and over & 7.7 & 7.8 & 7.8 & 7.8 & 8.5 & 7.5 & 6.3 \\
\hline \multicolumn{8}{|l|}{ Projections ${ }^{1}$} \\
\hline 2006 & 60,587 & 58,846 & 53,729 & 50,763 & 2,966 & 5,117 & 1,742 \\
\hline 2011 & 62,761 & 60,950 & 55,744 & 52,706 & 3,038 & 5,206 & 1,812 \\
\hline 2016 & 64,975 & 63,107 & 57,837 & 54,724 & 3,113 & 5,270 & 1,868 \\
\hline 2021 & 67,191 & 65,269 & 59,943 & 56,757 & 3,186 & 5,326 & 1,922 \\
\hline 2026 & 69,260 & 67,294 & 61,931 & 58,682 & 3,248 & 5,363 & 1,966 \\
\hline 2031 & 71,100 & 69,101 & 63,727 & 60,432 & 3,296 & 5,374 & 1,999 \\
\hline \multicolumn{8}{|c|}{2031 by age group (percentages) } \\
\hline $0-4$ & 5.5 & 5.5 & 5.6 & 5.6 & 5.1 & 4.7 & 5.7 \\
\hline $5-15$ & 12.4 & 12.4 & 12.5 & 12.5 & 12.1 & 11.2 & 13.4 \\
\hline $16-44$ & 36.4 & 36.4 & 36.6 & 36.8 & 33.7 & 34.3 & 35.5 \\
\hline $45-64^{2}$ & 23.4 & 23.4 & 23.3 & 23.3 & 23.5 & 24.4 & 23.9 \\
\hline $65-74^{2}$ & 10.6 & 10.6 & 10.5 & 10.4 & 12.0 & 12.4 & 10.7 \\
\hline 75 and over & 11.6 & 11.6 & 11.5 & 11.4 & 13.7 & 12.9 & 10.9 \\
\hline
\end{tabular}

1 National projections based on mid-2006 population estimates.

2 Between 2010 and 2020, state pension age will change from 65 years for men and 60 years for women to 65 years for both sexes.

Between 2024 and 2026, state pension age will increase from 65 years to 66 years for both men and women. 


\section{Table 1.3}

Population: subnational

Government Office Regions of England

\begin{tabular}{l|}
\hline Mid-year \\
\hline Estimates
\end{tabular}

\begin{tabular}{ll} 
& \\
\hline Estimates & 2,679 \\
1971 & 2,671
\end{tabular}

\section{6}

1981

1981

1991

1993

1993
1994

1994

1996

1997

1998

1999

2001

2002

2003

2004

2005

2007

\begin{tabular}{|c|c|c|c|c|c|c|c|c|}
\hline \multicolumn{9}{|c|}{ Numbers (thousands) and percentage age distributio } \\
\hline $\begin{array}{l}\text { North } \\
\text { East }\end{array}$ & $\begin{array}{l}\text { North } \\
\text { West }\end{array}$ & $\begin{array}{l}\text { Yorkshire } \\
\text { and The } \\
\text { Humber }\end{array}$ & $\begin{array}{c}\text { East } \\
\text { Midlands }\end{array}$ & $\begin{array}{c}\text { West } \\
\text { Midlands }\end{array}$ & East & London & $\begin{array}{c}\text { South } \\
\text { East }\end{array}$ & $\begin{array}{l}\text { South } \\
\text { West }\end{array}$ \\
\hline 2,679 & 7,108 & 4,902 & 3,652 & 5,146 & 4,454 & 7,529 & 6,830 & 4,112 \\
\hline 2,671 & 7,043 & 4,924 & 3,774 & 5,178 & 4,672 & 7,089 & 7,029 & 4,280 \\
\hline 2,636 & 6,940 & 4,918 & 3,853 & 5,187 & 4,854 & 6,806 & 7,245 & 4,381 \\
\hline 2,594 & 6,833 & 4,884 & 3,908 & 5,180 & 4,999 & 6,774 & 7,468 & 4,548 \\
\hline 2,587 & 6,843 & 4,936 & 4,011 & 5,230 & 5,121 & 6,829 & 7,629 & 4,688 \\
\hline 2,594 & 6,847 & 4,954 & 4,056 & 5,246 & 5,154 & 6,844 & 7,673 & 4,734 \\
\hline 2,589 & 6,839 & 4,960 & 4,072 & 5,249 & 5,178 & 6,874 & 7,712 & 4,757 \\
\hline 2,583 & 6,828 & 4,961 & 4,092 & 5,257 & 5,206 & 6,913 & 7,763 & 4,782 \\
\hline 2,576 & 6,810 & 4,961 & 4,108 & 5,263 & 5,233 & 6,974 & 7,800 & 4,793 \\
\hline 2,568 & 6,794 & 4,958 & 4,120 & 5,262 & 5,267 & 7,015 & 7,853 & 4,827 \\
\hline 2,561 & 6,792 & 4,958 & 4,133 & 5,271 & 5,302 & 7,065 & 7,889 & 4,849 \\
\hline 2,550 & 6,773 & 4,956 & 4,152 & 5,272 & 5,339 & 7,154 & 7,955 & 4,881 \\
\hline 2,543 & 6,774 & 4,959 & 4,168 & 5,270 & 5,375 & 7,237 & 7,991 & 4,917 \\
\hline 2,540 & 6,773 & 4,977 & 4,190 & 5,281 & 5,400 & 7,322 & 8,023 & 4,943 \\
\hline 2,541 & 6,778 & 5,002 & 4,222 & 5,295 & 5,433 & 7,362 & 8,047 & 4,973 \\
\hline 2,541 & 6,800 & 5,028 & 4,254 & 5,312 & 5,475 & 7,364 & 8,087 & 5,005 \\
\hline 2,542 & 6,820 & 5,064 & 4,291 & 5,327 & 5,511 & 7,389 & 8,125 & 5,042 \\
\hline 2,550 & 6,840 & 5,108 & 4,328 & 5,351 & 5,563 & 7,456 & 8,185 & 5,087 \\
\hline 2,556 & 6,853 & 5,142 & 4,364 & 5,367 & 5,607 & 7,512 & 8,238 & 5,124 \\
\hline 2,564 & 6,864 & 5,177 & 4,400 & 5,382 & 5,661 & 7,557 & 8,309 & 5,178 \\
\hline
\end{tabular}

\begin{tabular}{|c|c|c|c|c|c|c|c|c|}
\hline \multicolumn{9}{|c|}{ Numbers (thousands) and percentage age distributio } \\
\hline $\begin{array}{l}\text { North } \\
\text { East }\end{array}$ & $\begin{array}{l}\text { North } \\
\text { West }\end{array}$ & $\begin{array}{l}\text { Yorkshire } \\
\text { and The } \\
\text { Humber }\end{array}$ & $\begin{array}{c}\text { East } \\
\text { Midlands }\end{array}$ & $\begin{array}{c}\text { West } \\
\text { Midlands }\end{array}$ & East & London & $\begin{array}{c}\text { South } \\
\text { East }\end{array}$ & $\begin{array}{l}\text { South } \\
\text { West }\end{array}$ \\
\hline 2,679 & 7,108 & 4,902 & 3,652 & 5,146 & 4,454 & 7,529 & 6,830 & 4,112 \\
\hline 2,671 & 7,043 & 4,924 & 3,774 & 5,178 & 4,672 & 7,089 & 7,029 & 4,280 \\
\hline 2,636 & 6,940 & 4,918 & 3,853 & 5,187 & 4,854 & 6,806 & 7,245 & 4,381 \\
\hline 2,594 & 6,833 & 4,884 & 3,908 & 5,180 & 4,999 & 6,774 & 7,468 & 4,548 \\
\hline 2,587 & 6,843 & 4,936 & 4,011 & 5,230 & 5,121 & 6,829 & 7,629 & 4,688 \\
\hline 2,594 & 6,847 & 4,954 & 4,056 & 5,246 & 5,154 & 6,844 & 7,673 & 4,734 \\
\hline 2,589 & 6,839 & 4,960 & 4,072 & 5,249 & 5,178 & 6,874 & 7,712 & 4,757 \\
\hline 2,583 & 6,828 & 4,961 & 4,092 & 5,257 & 5,206 & 6,913 & 7,763 & 4,782 \\
\hline 2,576 & 6,810 & 4,961 & 4,108 & 5,263 & 5,233 & 6,974 & 7,800 & 4,793 \\
\hline 2,568 & 6,794 & 4,958 & 4,120 & 5,262 & 5,267 & 7,015 & 7,853 & 4,827 \\
\hline 2,561 & 6,792 & 4,958 & 4,133 & 5,271 & 5,302 & 7,065 & 7,889 & 4,849 \\
\hline 2,550 & 6,773 & 4,956 & 4,152 & 5,272 & 5,339 & 7,154 & 7,955 & 4,881 \\
\hline 2,543 & 6,774 & 4,959 & 4,168 & 5,270 & 5,375 & 7,237 & 7,991 & 4,917 \\
\hline 2,540 & 6,773 & 4,977 & 4,190 & 5,281 & 5,400 & 7,322 & 8,023 & 4,943 \\
\hline 2,541 & 6,778 & 5,002 & 4,222 & 5,295 & 5,433 & 7,362 & 8,047 & 4,973 \\
\hline 2,541 & 6,800 & 5,028 & 4,254 & 5,312 & 5,475 & 7,364 & 8,087 & 5,005 \\
\hline 2,542 & 6,820 & 5,064 & 4,291 & 5,327 & 5,511 & 7,389 & 8,125 & 5,042 \\
\hline 2,550 & 6,840 & 5,108 & 4,328 & 5,351 & 5,563 & 7,456 & 8,185 & 5,087 \\
\hline 2,556 & 6,853 & 5,142 & 4,364 & 5,367 & 5,607 & 7,512 & 8,238 & 5,124 \\
\hline 2,564 & 6,864 & 5,177 & 4,400 & 5,382 & 5,661 & 7,557 & 8,309 & 5,178 \\
\hline
\end{tabular}

\begin{tabular}{|c|c|c|c|c|c|c|c|c|}
\hline \multicolumn{9}{|c|}{ Numbers (thousands) and percentage age distributic } \\
\hline $\begin{array}{c}\text { North } \\
\text { East }\end{array}$ & $\begin{array}{l}\text { North } \\
\text { West }\end{array}$ & $\begin{array}{l}\text { Yorkshire } \\
\text { and The } \\
\text { Humber }\end{array}$ & $\begin{array}{c}\text { East } \\
\text { Midlands }\end{array}$ & $\begin{array}{c}\text { West } \\
\text { Midlands }\end{array}$ & East & London & $\begin{array}{c}\text { South } \\
\text { East }\end{array}$ & $\begin{array}{l}\text { South } \\
\text { West }\end{array}$ \\
\hline 2,679 & 7,108 & 4,902 & 3,652 & 5,146 & 4,454 & 7,529 & 6,830 & 4,112 \\
\hline 2,671 & 7,043 & 4,924 & 3,774 & 5,178 & 4,672 & 7,089 & 7,029 & 4,280 \\
\hline 2,636 & 6,940 & 4,918 & 3,853 & 5,187 & 4,854 & 6,806 & 7,245 & 4,381 \\
\hline 2,594 & 6,833 & 4,884 & 3,908 & 5,180 & 4,999 & 6,774 & 7,468 & 4,548 \\
\hline 2,587 & 6,843 & 4,936 & 4,011 & 5,230 & 5,121 & 6,829 & 7,629 & 4,688 \\
\hline 2,594 & 6,847 & 4,954 & 4,056 & 5,246 & 5,154 & 6,844 & 7,673 & 4,734 \\
\hline 2,589 & 6,839 & 4,960 & 4,072 & 5,249 & 5,178 & 6,874 & 7,712 & 4,757 \\
\hline 2,583 & 6,828 & 4,961 & 4,092 & 5,257 & 5,206 & 6,913 & 7,763 & 4,782 \\
\hline 2,576 & 6,810 & 4,961 & 4,108 & 5,263 & 5,233 & 6,974 & 7,800 & 4,793 \\
\hline 2,568 & 6,794 & 4,958 & 4,120 & 5,262 & 5,267 & 7,015 & 7,853 & 4,827 \\
\hline 2,561 & 6,792 & 4,958 & 4,133 & 5,271 & 5,302 & 7,065 & 7,889 & 4,849 \\
\hline 2,550 & 6,773 & 4,956 & 4,152 & 5,272 & 5,339 & 7,154 & 7,955 & 4,881 \\
\hline 2,543 & 6,774 & 4,959 & 4,168 & 5,270 & 5,375 & 7,237 & 7,991 & 4,917 \\
\hline 2,540 & 6,773 & 4,977 & 4,190 & 5,281 & 5,400 & 7,322 & 8,023 & 4,943 \\
\hline 2,541 & 6,778 & 5,002 & 4,222 & 5,295 & 5,433 & 7,362 & 8,047 & 4,973 \\
\hline 2,541 & 6,800 & 5,028 & 4,254 & 5,312 & 5,475 & 7,364 & 8,087 & 5,005 \\
\hline 2,542 & 6,820 & 5,064 & 4,291 & 5,327 & 5,511 & 7,389 & 8,125 & 5,042 \\
\hline 2,550 & 6,840 & 5,108 & 4,328 & 5,351 & 5,563 & 7,456 & 8,185 & 5,087 \\
\hline 2,556 & 6,853 & 5,142 & 4,364 & 5,367 & 5,607 & 7,512 & 8,238 & 5,124 \\
\hline 2,564 & 6,864 & 5,177 & 4,400 & 5,382 & 5,661 & 7,557 & 8,309 & 5,178 \\
\hline
\end{tabular}

Numbers (thousands) and percentage age distribution

2007 by age group (percentages)
$0-4$
5-15
$16-44$
$45-64 \mathrm{M} / 59 \mathrm{~F}$
$65 \mathrm{M} / 60 \mathrm{~F}-74$
75 and over

$\begin{array}{rrr}5.5 & 5.9 & 5.8 \\ 12.6 & 13.2 & 13.0 \\ 38.9 & 39.4 & 40.3 \\ 23.2 & 22.3 & 22.0 \\ 11.9 & 11.5 & 11.3 \\ 8.0 & 7.7 & 7.6\end{array}$

5.6
12.9
39.4
22.7
11.6
7.8

$\begin{array}{rr}6.1 & 5.9 \\ 13.4 & 13.2 \\ 39.1 & 38.6 \\ 22.0 & 22.4 \\ 11.6 & 11.7 \\ 7.9 & 8.2\end{array}$

$\begin{array}{rr}7.0 & 5.8 \\ 12.2 & 13.2 \\ 48.3 & 38.9 \\ 18.6 & 22.5 \\ 8.1 & 11.3 \\ 5.7 & 8.3\end{array}$

5.2
12.5
37.1
22.9
12.6
9.5

\section{Projections $^{1}$}

2006
2011
2016
2021
2026
2031

$\begin{array}{ll}2,556 & 6,853 \\ 2,594 & 7,014 \\ 2,638 & 7,193 \\ 2,685 & 7,377 \\ 2,730 & 7,546 \\ 2,769 & 7,696\end{array}$

$\begin{array}{lll}6,853 & 5,142 & 4,364 \\ 7,014 & 5,377 & 4,591 \\ 7,193 & 5,621 & 4,825 \\ 7,377 & 5,866 & 5,060 \\ 7,546 & 6,101 & 5,286 \\ 7,696 & 6,319 & 5,491\end{array}$

5,367
5,506
5,662
5,824
5,977
6,114

$\begin{array}{ll}5,607 & 7,512 \\ 5,890 & 7,817 \\ 6,179 & 8,114 \\ 6,471 & 8,390 \\ 6,747 & 8,633 \\ 6,997 & 8,858\end{array}$

\section{8,238}

8,238
8,550

$\begin{array}{ll}8,550 & 5,368 \\ 8,871 & 5,620\end{array}$

$\begin{array}{ll}8,871 & 5,620 \\ 9,202 & 5,882\end{array}$

$\begin{array}{ll}9,202 & 5,882 \\ 9,523 & 6,139\end{array}$

2031 by age group (percentages)

$0-4$
$5-15$
$16-44$

$\begin{array}{rrrr}5.2 & 5.5 & 5.6 & 5.3 \\ 12.1 & 12.6 & 12.5 & 12.3 \\ 35.6 & 36.3 & 37.7 & 35 . \\ 23.0 & 23.2 & 23.0 & 23.8 \\ 11.7 & 10.9 & 10.2 & 11.0 \\ 12.4 & 11.5 & 10.9 & 12.0\end{array}$

$\begin{array}{rr}5.3 & \\ 12.3 & 13.2 \\ 35.6 & 35.7 \\ 23.8 & 22.8 \\ 11.0 & 10.5 \\ 12.0 & 11.9\end{array}$

$\begin{array}{rrrrr}5.9 & 5.5 & 6.7 & 5.5 & 5.0 \\ 13.2 & 12.6 & 12.6 & 12.7 & 11.8 \\ 35.7 & 34.9 & 43.7 & 35.1 & 33.9 \\ 22.8 & 23.7 & 22.9 & 23.6 & 23.7 \\ 10.5 & 10.9 & 7.4 & 10.8 & 11.8 \\ 11.9 & 12.4 & 6.7 & 12.4 & 13.9\end{array}$

1 These projections are based on the 2006 population estimates and are consistent with the 2006-based national projections produced by the Office for National Statistics.

2 Between 2010 and 2020, state pension age will change from 65 years for men and 60 years for women to 65 years for both sexes.

Between 2024 and 2026, state pension age will increase from 65 years to 66 years for both men and women. 


\begin{tabular}{|c|c|c|c|c|c|c|c|c|c|c|c|c|c|c|c|c|}
\hline Table 1.4 & \multicolumn{16}{|c|}{ Population: age and sex } \\
\hline \multicolumn{15}{|c|}{ Constituent countries of the United Kingdom } & \multicolumn{2}{|c|}{ Numbers (thousands) } \\
\hline & & \multicolumn{15}{|c|}{ Age group } \\
\hline Mid-year & All ages & Under 1 & $1-4$ & $5-14$ & $15-24$ & $25-34$ & $35-44$ & $45-59$ & $60-64$ & $65-74$ & $75-84$ & $85-89$ & $\begin{array}{c}90 \text { and } \\
\text { over }\end{array}$ & $\begin{array}{c}\text { Under } \\
16\end{array}$ & $\begin{array}{c}16- \\
64 \mathrm{M} / 59 \mathrm{~F}^{\prime}\end{array}$ & $\begin{array}{l}65 \mathrm{M} / 60 \mathrm{~F}^{1} \\
\text { and over }\end{array}$ \\
\hline $\begin{array}{l}\text { United Kingd } \\
\text { Persons } \\
1981 \\
1986 \\
1991 \\
1996\end{array}$ & $\begin{array}{l}56,357 \\
56,684 \\
57,439 \\
58,164\end{array}$ & $\begin{array}{l}730 \\
748 \\
790 \\
719\end{array}$ & $\begin{array}{l}2,726 \\
2,886 \\
3,077 \\
3,019\end{array}$ & $\begin{array}{l}8,147 \\
7,143 \\
7,141 \\
7,544\end{array}$ & $\begin{array}{l}9,019 \\
9,200 \\
8,168 \\
7,231\end{array}$ & $\begin{array}{l}8,010 \\
8,007 \\
8,898 \\
9,131\end{array}$ & $\begin{array}{l}6,774 \\
7,711 \\
7,918 \\
7,958\end{array}$ & $\begin{array}{r}9,540 \\
9,212 \\
9,500 \\
10,553\end{array}$ & $\begin{array}{l}2,935 \\
3,069 \\
2,888 \\
2,785\end{array}$ & $\begin{array}{l}5,195 \\
5,020 \\
5,067 \\
5,066\end{array}$ & $\begin{array}{l}2,677 \\
2,971 \\
3,119 \\
3,129\end{array}$ & $\begin{array}{l}716 \\
626 \\
711\end{array}$ & 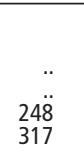 & $\begin{array}{l}12,543 \\
11,645 \\
11,685 \\
12,018\end{array}$ & $\begin{array}{l}33,780 \\
34,725 \\
35,197 \\
35,498\end{array}$ & $\begin{array}{l}10,035 \\
10,313 \\
10,557 \\
10,649\end{array}$ \\
\hline $\begin{array}{l}2000 \\
2001 \\
2002 \\
2003\end{array}$ & $\begin{array}{l}58,886 \\
59,113 \\
59,323 \\
59,557\end{array}$ & $\begin{array}{l}682 \\
663 \\
661 \\
680\end{array}$ & $\begin{array}{l}2,869 \\
2,819 \\
2,753 \\
2,706\end{array}$ & $\begin{array}{l}7,652 \\
7,624 \\
7,603 \\
7,546\end{array}$ & $\begin{array}{l}7,139 \\
7,261 \\
7,400 \\
7,573\end{array}$ & $\begin{array}{l}8,646 \\
8,475 \\
8,264 \\
8,084\end{array}$ & $\begin{array}{l}8,678 \\
8,846 \\
9,004 \\
9,105\end{array}$ & $\begin{array}{l}11,011 \\
11,168 \\
11,307 \\
11,412\end{array}$ & $\begin{array}{l}2,900 \\
2,884 \\
2,892 \\
2,949\end{array}$ & $\begin{array}{l}4,940 \\
4,947 \\
4,967 \\
5,001\end{array}$ & $\begin{array}{l}3,249 \\
3,296 \\
3,344 \\
3,398\end{array}$ & $\begin{array}{l}755 \\
753 \\
738 \\
706\end{array}$ & $\begin{array}{l}364 \\
377 \\
388 \\
399\end{array}$ & $\begin{array}{l}11,959 \\
11,863 \\
11,785 \\
11,720\end{array}$ & $\begin{array}{l}36,138 \\
36,406 \\
36,622 \\
36,826\end{array}$ & $\begin{array}{l}10,788 \\
10,845 \\
10,916 \\
11,012\end{array}$ \\
\hline $\begin{array}{l}2004 \\
2005 \\
2006 \\
2007\end{array}$ & $\begin{array}{l}59,846 \\
60,238 \\
60,587 \\
60,975\end{array}$ & $\begin{array}{l}705 \\
716 \\
732 \\
756\end{array}$ & $\begin{array}{l}2,686 \\
2,713 \\
2,765 \\
2,837\end{array}$ & $\begin{array}{l}7,475 \\
7,373 \\
7,241 \\
7,128\end{array}$ & $\begin{array}{l}7,739 \\
7,886 \\
8,020 \\
8,156\end{array}$ & $\begin{array}{l}7,954 \\
7,935 \\
7,896 \\
7,859\end{array}$ & $\begin{array}{l}9,185 \\
9,245 \\
9,262 \\
9,248\end{array}$ & $\begin{array}{l}11,507 \\
11,616 \\
11,744 \\
11,728\end{array}$ & $\begin{array}{l}3,027 \\
3,114 \\
3,240 \\
3,483\end{array}$ & $\begin{array}{l}5,028 \\
5,046 \\
5,029 \\
5,058\end{array}$ & $\begin{array}{l}3,431 \\
3,420 \\
3,416 \\
3,424\end{array}$ & $\begin{array}{l}702 \\
755 \\
820 \\
873\end{array}$ & $\begin{array}{l}409 \\
419 \\
423 \\
425\end{array}$ & $\begin{array}{l}11,645 \\
11,589 \\
11,537 \\
11,509\end{array}$ & $\begin{array}{l}37,083 \\
37,418 \\
37,707 \\
37,904\end{array}$ & $\begin{array}{l}11,117 \\
11,232 \\
11,344 \\
11,562\end{array}$ \\
\hline $\begin{array}{l}\text { Males } \\
1981 \\
1986 \\
1991 \\
1996\end{array}$ & $\begin{array}{l}27,412 \\
27,542 \\
27,909 \\
28,287\end{array}$ & $\begin{array}{l}374 \\
384 \\
403 \\
369\end{array}$ & $\begin{array}{l}1,400 \\
1,478 \\
1,572 \\
1,547\end{array}$ & $\begin{array}{l}4,184 \\
3,664 \\
3,655 \\
3,857\end{array}$ & $\begin{array}{l}4,596 \\
4,663 \\
4,146 \\
3,652\end{array}$ & $\begin{array}{l}4,035 \\
4,022 \\
4,432 \\
4,540\end{array}$ & $\begin{array}{l}3,409 \\
3,864 \\
3,949 \\
3,954\end{array}$ & $\begin{array}{l}4,711 \\
4,572 \\
4,732 \\
5,244\end{array}$ & $\begin{array}{l}1,376 \\
1,463 \\
1,390 \\
1,360\end{array}$ & $\begin{array}{l}2,264 \\
2,206 \\
2,272 \\
2,311\end{array}$ & $\begin{array}{r}922 \\
1,060 \\
1,146 \\
1,187\end{array}$ & $\begin{array}{l}16 \ddot{6} \\
166 \\
201\end{array}$ & $\begin{array}{l}46 \\
65\end{array}$ & $\begin{array}{l}6,439 \\
5,968 \\
5,976 \\
6,148\end{array}$ & $\begin{array}{l}17,646 \\
18,142 \\
18,303 \\
18,375\end{array}$ & $\begin{array}{l}3,327 \\
3,432 \\
3,630 \\
3,764\end{array}$ \\
\hline $\begin{array}{l}2000 \\
2001 \\
2002 \\
2003\end{array}$ & $\begin{array}{l}28,690 \\
28,832 \\
28,964 \\
29,109\end{array}$ & $\begin{array}{l}350 \\
338 \\
338 \\
349\end{array}$ & $\begin{array}{l}1,469 \\
1,445 \\
1,408 \\
1,384\end{array}$ & $\begin{array}{l}3,920 \\
3,906 \\
3,897 \\
3,868\end{array}$ & $\begin{array}{l}3,606 \\
3,672 \\
3,758 \\
3,855\end{array}$ & $\begin{array}{l}4,292 \\
4,215 \\
4,114 \\
4,024\end{array}$ & $\begin{array}{l}4,298 \\
4,382 \\
4,462 \\
4,514\end{array}$ & $\begin{array}{l}5,457 \\
5,534 \\
5,594 \\
5,646\end{array}$ & $\begin{array}{l}1,420 \\
1,412 \\
1,414 \\
1,440\end{array}$ & $\begin{array}{l}2,294 \\
2,308 \\
2,325 \\
2,347\end{array}$ & $\begin{array}{l}1,278 \\
1,308 \\
1,338 \\
1,369\end{array}$ & $\begin{array}{l}225 \\
227 \\
226 \\
219\end{array}$ & $\begin{array}{l}81 \\
85 \\
89 \\
94\end{array}$ & $\begin{array}{l}6,128 \\
6,077 \\
6,037 \\
6,006\end{array}$ & $\begin{array}{l}18,685 \\
18,827 \\
18,949 \\
19,075\end{array}$ & $\begin{array}{l}3,878 \\
3,928 \\
3,978 \\
4,028\end{array}$ \\
\hline $\begin{array}{l}2004 \\
2005 \\
2006 \\
2007\end{array}$ & $\begin{array}{l}29,278 \\
29,497 \\
29,694 \\
29,916\end{array}$ & $\begin{array}{l}362 \\
367 \\
374 \\
387\end{array}$ & $\begin{array}{l}1,376 \\
1,389 \\
1,416 \\
1,453\end{array}$ & $\begin{array}{l}3,832 \\
3,781 \\
3,709 \\
3,649\end{array}$ & $\begin{array}{l}3,953 \\
4,030 \\
4,108 \\
4,193\end{array}$ & $\begin{array}{l}3,960 \\
3,952 \\
3,940 \\
3,936\end{array}$ & $\begin{array}{l}4,546 \\
4,581 \\
4,586 \\
4,578\end{array}$ & $\begin{array}{l}5,691 \\
5,745 \\
5,804 \\
5,786\end{array}$ & $\begin{array}{l}1,479 \\
1,522 \\
1,584 \\
1,701\end{array}$ & $\begin{array}{l}2,365 \\
2,380 \\
2,379 \\
2,398\end{array}$ & $\begin{array}{l}1,392 \\
1,400 \\
1,413 \\
1,432\end{array}$ & $\begin{array}{l}223 \\
247 \\
273 \\
295\end{array}$ & $\begin{array}{r}98 \\
103 \\
106 \\
108\end{array}$ & $\begin{array}{l}5,971 \\
5,941 \\
5,912 \\
5,895\end{array}$ & $\begin{array}{l}19,229 \\
19,426 \\
19,611 \\
19,789\end{array}$ & $\begin{array}{l}4,078 \\
4,130 \\
4,171 \\
4,233\end{array}$ \\
\hline $\begin{array}{l}\text { Females } \\
1981 \\
1986 \\
1991 \\
1996\end{array}$ & $\begin{array}{l}28,946 \\
29,142 \\
29,530 \\
29,877\end{array}$ & $\begin{array}{l}356 \\
364 \\
387 \\
350\end{array}$ & $\begin{array}{l}1,327 \\
1,408 \\
1,505 \\
1,472\end{array}$ & $\begin{array}{l}3,963 \\
3,480 \\
3,487 \\
3,687\end{array}$ & $\begin{array}{l}4,423 \\
4,538 \\
4,021 \\
3,579\end{array}$ & $\begin{array}{l}3,975 \\
3,985 \\
4,466 \\
4,591\end{array}$ & $\begin{array}{l}3,365 \\
3,847 \\
3,968 \\
4,005\end{array}$ & $\begin{array}{l}4,829 \\
4,639 \\
4,769 \\
5,309\end{array}$ & $\begin{array}{l}1,559 \\
1,606 \\
1,498 \\
1,426\end{array}$ & $\begin{array}{l}2,931 \\
2,814 \\
2,795 \\
2,755\end{array}$ & $\begin{array}{l}1,756 \\
1,911 \\
1,972 \\
1,942\end{array}$ & $\begin{array}{l}550 \\
460 \\
509\end{array}$ & $\begin{array}{l}202 \\
252\end{array}$ & $\begin{array}{l}6,104 \\
5,678 \\
5,709 \\
5,870\end{array}$ & $\begin{array}{l}16,134 \\
16,583 \\
16,894 \\
17,123\end{array}$ & $\begin{array}{l}6,708 \\
6,881 \\
6,927 \\
6,885\end{array}$ \\
\hline $\begin{array}{l}2000 \\
2001 \\
2002 \\
2003\end{array}$ & $\begin{array}{l}30,196 \\
30,281 \\
30,359 \\
30,449\end{array}$ & $\begin{array}{l}333 \\
324 \\
323 \\
331\end{array}$ & $\begin{array}{l}1,399 \\
1,375 \\
1,346 \\
1,322\end{array}$ & $\begin{array}{l}3,732 \\
3,718 \\
3,706 \\
3,678\end{array}$ & $\begin{array}{l}3,533 \\
3,589 \\
3,642 \\
3,718\end{array}$ & $\begin{array}{l}4,353 \\
4,260 \\
4,150 \\
4,060\end{array}$ & $\begin{array}{l}4,380 \\
4,465 \\
4,542 \\
4,590\end{array}$ & $\begin{array}{l}5,554 \\
5,634 \\
5,713 \\
5,766\end{array}$ & $\begin{array}{l}1,481 \\
1,473 \\
1,478 \\
1,509\end{array}$ & $\begin{array}{l}2,646 \\
2,640 \\
2,642 \\
2,654\end{array}$ & $\begin{array}{l}1,971 \\
1,987 \\
2,006 \\
2,029\end{array}$ & $\begin{array}{l}530 \\
526 \\
513 \\
487\end{array}$ & $\begin{array}{l}283 \\
292 \\
299 \\
305\end{array}$ & $\begin{array}{l}5,832 \\
5,786 \\
5,748 \\
5,714\end{array}$ & $\begin{array}{l}17,453 \\
17,579 \\
17,673 \\
17,751\end{array}$ & $\begin{array}{l}6,911 \\
6,917 \\
6,938 \\
6,984\end{array}$ \\
\hline $\begin{array}{l}2004 \\
2005 \\
2006 \\
2007\end{array}$ & $\begin{array}{l}30,568 \\
30,741 \\
30,893 \\
31,059\end{array}$ & $\begin{array}{l}343 \\
349 \\
357 \\
368\end{array}$ & $\begin{array}{l}1,310 \\
1,324 \\
1,349 \\
1,383\end{array}$ & $\begin{array}{l}3,642 \\
3,592 \\
3,532 \\
3,480\end{array}$ & $\begin{array}{l}3,785 \\
3,856 \\
3,912 \\
3,963\end{array}$ & $\begin{array}{l}3,993 \\
3,983 \\
3,956 \\
3,924\end{array}$ & $\begin{array}{l}4,639 \\
4,663 \\
4,675 \\
4,670\end{array}$ & $\begin{array}{l}5,816 \\
5,871 \\
5,940 \\
5,942\end{array}$ & $\begin{array}{l}1,548 \\
1,591 \\
1,656 \\
1,782\end{array}$ & $\begin{array}{l}2,662 \\
2,666 \\
2,650 \\
2,660\end{array}$ & $\begin{array}{l}2,040 \\
2,020 \\
2,002 \\
1,992\end{array}$ & $\begin{array}{l}479 \\
509 \\
547 \\
578\end{array}$ & $\begin{array}{l}310 \\
316 \\
317 \\
317\end{array}$ & $\begin{array}{l}5,674 \\
5,647 \\
5,625 \\
5,615\end{array}$ & $\begin{array}{l}17,854 \\
17,992 \\
18,096 \\
18,116\end{array}$ & $\begin{array}{l}7,039 \\
7,102 \\
7,172 \\
7,329\end{array}$ \\
\hline $\begin{array}{l}\text { England anc } \\
\text { Persons } \\
1981 \\
1986 \\
1991 \\
1996\end{array}$ & $\begin{array}{l}49,634 \\
49,999 \\
50,748 \\
51,410\end{array}$ & $\begin{array}{l}634 \\
654 \\
698 \\
637\end{array}$ & $\begin{array}{l}2,372 \\
2,522 \\
2,713 \\
2,668\end{array}$ & $\begin{array}{l}7,085 \\
6,226 \\
6,248 \\
6,636\end{array}$ & $\begin{array}{l}7,873 \\
8,061 \\
7,165 \\
6,336\end{array}$ & $\begin{array}{l}7,086 \\
7,052 \\
7,862 \\
8,076\end{array}$ & $\begin{array}{l}5,996 \\
6,856 \\
7,022 \\
7,017\end{array}$ & $\begin{array}{l}8,433 \\
8,136 \\
8,407 \\
9,363\end{array}$ & $\begin{array}{l}2,607 \\
2,725 \\
2,553 \\
2,457\end{array}$ & $\begin{array}{l}4,619 \\
4,470 \\
4,506 \\
4,496\end{array}$ & $\begin{array}{l}2,388 \\
2,655 \\
2,790 \\
2,801\end{array}$ & $\begin{array}{l}383 \\
461 \\
561 \\
639\end{array}$ & $\begin{array}{l}157 \\
182 \\
223 \\
285\end{array}$ & $\begin{array}{l}10,910 \\
10,161 \\
10,247 \\
10,584\end{array}$ & $\begin{array}{l}29,796 \\
30,647 \\
31,100 \\
31,353\end{array}$ & $\begin{array}{l}8,928 \\
9,190 \\
9,400 \\
9,474\end{array}$ \\
\hline $\begin{array}{l}2000 \\
2001 \\
2002 \\
2003\end{array}$ & $\begin{array}{l}52,140 \\
52,360 \\
52,572 \\
52,797\end{array}$ & $\begin{array}{l}607 \\
589 \\
589 \\
607\end{array}$ & $\begin{array}{l}2,544 \\
2,502 \\
2,445 \\
2,404\end{array}$ & $\begin{array}{l}6,757 \\
6,740 \\
6,728 \\
6,682\end{array}$ & $\begin{array}{l}6,275 \\
6,387 \\
6,518 \\
6,679\end{array}$ & $\begin{array}{l}7,682 \\
7,536 \\
7,357 \\
7,203\end{array}$ & $\begin{array}{l}7,661 \\
7,616 \\
7,964 \\
8,058\end{array}$ & $\begin{array}{r}9,764 \\
9,898 \\
10,018 \\
10,104\end{array}$ & $\begin{array}{l}2,564 \\
2,549 \\
2,555 \\
2,606\end{array}$ & $\begin{array}{l}4,372 \\
4,377 \\
4,394 \\
4,422\end{array}$ & $\begin{array}{l}2,907 \\
2,947 \\
2,989 \\
3,037\end{array}$ & $\begin{array}{l}680 \\
677 \\
664 \\
634\end{array}$ & $\begin{array}{l}328 \\
340 \\
351 \\
360\end{array}$ & $\begin{array}{l}10,572 \\
10,495 \\
10,437 \\
10,388\end{array}$ & $\begin{array}{l}31,977 \\
32,226 \\
32,435 \\
32,626\end{array}$ & $\begin{array}{l}9,591 \\
9,639 \\
9,700 \\
9,783\end{array}$ \\
\hline $\begin{array}{l}2004 \\
2005 \\
2006 \\
2007\end{array}$ & $\begin{array}{l}53,057 \\
53,419 \\
53,729 \\
54,072\end{array}$ & $\begin{array}{l}629 \\
639 \\
653 \\
675\end{array}$ & $\begin{array}{l}2,390 \\
2,415 \\
2,462 \\
2,528\end{array}$ & $\begin{array}{l}6,618 \\
6,528 \\
6,412 \\
6,314\end{array}$ & $\begin{array}{l}6,836 \\
6,974 \\
7,095 \\
7,219\end{array}$ & $\begin{array}{l}7,090 \\
7,078 \\
7,040 \\
6,999\end{array}$ & $\begin{array}{l}8,133 \\
8,194 \\
8,213 \\
8,209\end{array}$ & $\begin{array}{l}10,177 \\
10,264 \\
10,369 \\
10,347\end{array}$ & $\begin{array}{l}2,675 \\
2,757 \\
2,874 \\
3,092\end{array}$ & $\begin{array}{l}4,445 \\
4,461 \\
4,444 \\
4,468\end{array}$ & $\begin{array}{l}3,063 \\
3,052 \\
3,045 \\
3,049\end{array}$ & $\begin{array}{l}632 \\
680 \\
740 \\
787\end{array}$ & $\begin{array}{l}370 \\
379 \\
382 \\
385\end{array}$ & $\begin{array}{l}10,326 \\
10,278 \\
10,235 \\
10,212\end{array}$ & $\begin{array}{l}32,856 \\
33,164 \\
33,417 \\
33,588\end{array}$ & $\begin{array}{r}9,875 \\
9,977 \\
10,077 \\
10,271\end{array}$ \\
\hline $\begin{array}{l}\text { Males } \\
1981 \\
1986 \\
1991 \\
1996\end{array}$ & $\begin{array}{l}24,160 \\
24,311 \\
24,681 \\
25,030\end{array}$ & $\begin{array}{l}324 \\
335 \\
356 \\
327\end{array}$ & $\begin{array}{l}1,218 \\
1,292 \\
1,385 \\
1,368\end{array}$ & $\begin{array}{l}3,639 \\
3,194 \\
3,198 \\
3,393\end{array}$ & $\begin{array}{l}4,011 \\
4,083 \\
3,638 \\
3,202\end{array}$ & $\begin{array}{l}3,569 \\
3,542 \\
3,920 \\
4,020\end{array}$ & $\begin{array}{l}3,024 \\
3,438 \\
3,504 \\
3,489\end{array}$ & $\begin{array}{l}4,178 \\
4,053 \\
4,199 \\
4,659\end{array}$ & $\begin{array}{l}1,227 \\
1,302 \\
1,234 \\
1,205\end{array}$ & $\begin{array}{l}2,020 \\
1,972 \\
2,027 \\
2,059\end{array}$ & $\begin{array}{r}825 \\
951 \\
1,029 \\
1,067\end{array}$ & $\begin{array}{r}94 \\
115 \\
150 \\
182\end{array}$ & $\begin{array}{l}32 \\
35 \\
42 \\
59\end{array}$ & $\begin{array}{l}5,601 \\
5,208 \\
5,240 \\
5,416\end{array}$ & $\begin{array}{l}15,589 \\
16,031 \\
16,193 \\
16,247\end{array}$ & $\begin{array}{l}2,970 \\
3,072 \\
3,248 \\
3,367\end{array}$ \\
\hline $\begin{array}{l}2000 \\
2001 \\
2002 \\
2003\end{array}$ & $\begin{array}{l}25,438 \\
25,574 \\
25,704 \\
25,841\end{array}$ & $\begin{array}{l}311 \\
301 \\
301 \\
312\end{array}$ & $\begin{array}{l}1,303 \\
1,281 \\
1,249 \\
1,230\end{array}$ & $\begin{array}{l}3,462 \\
3,453 \\
3,448 \\
3,425\end{array}$ & $\begin{array}{l}3,172 \\
3,231 \\
3,311 \\
3,399\end{array}$ & $\begin{array}{l}3,823 \\
3,758 \\
3,672 \\
3,594\end{array}$ & $\begin{array}{l}3,802 \\
3,881 \\
3,957 \\
4,007\end{array}$ & $\begin{array}{l}4,842 \\
4,907 \\
4,958 \\
5,002\end{array}$ & $\begin{array}{l}1,259 \\
1,252 \\
1,253 \\
1,276\end{array}$ & $\begin{array}{l}2,040 \\
2,052 \\
2,067 \\
2,085\end{array}$ & $\begin{array}{l}1,148 \\
1,175 \\
1,202 \\
1,229\end{array}$ & $\begin{array}{l}204 \\
206 \\
204 \\
198\end{array}$ & $\begin{array}{l}73 \\
77 \\
81 \\
85\end{array}$ & $\begin{array}{l}5,416 \\
5,376 \\
5,346 \\
5,324\end{array}$ & $\begin{array}{l}16,556 \\
16,688 \\
16,804 \\
16,920\end{array}$ & $\begin{array}{l}3,466 \\
3,510 \\
3,554 \\
3,597\end{array}$ \\
\hline $\begin{array}{l}2004 \\
2005 \\
2006 \\
2007\end{array}$ & $\begin{array}{l}25,995 \\
26,197 \\
26,371 \\
26,569\end{array}$ & $\begin{array}{l}323 \\
327 \\
334 \\
346\end{array}$ & $\begin{array}{l}1,225 \\
1,237 \\
1,261 \\
1,295\end{array}$ & $\begin{array}{l}3,394 \\
3,348 \\
3,284 \\
3,231\end{array}$ & $\begin{array}{l}3,493 \\
3,565 \\
3,636 \\
3,715\end{array}$ & $\begin{array}{l}3,538 \\
3,530 \\
3,517 \\
3,508\end{array}$ & $\begin{array}{l}4,036 \\
4,073 \\
4,080 \\
4,076\end{array}$ & $\begin{array}{l}5,037 \\
5,080 \\
5,130 \\
5,110\end{array}$ & $\begin{array}{l}1,310 \\
1,351 \\
1,407 \\
1,511\end{array}$ & $\begin{array}{l}2,100 \\
2,113 \\
2,111 \\
2,127\end{array}$ & $\begin{array}{l}1,248 \\
1,256 \\
1,267 \\
1,283\end{array}$ & $\begin{array}{l}202 \\
224 \\
248 \\
268\end{array}$ & $\begin{array}{l}89 \\
94 \\
96 \\
99\end{array}$ & $\begin{array}{l}5,295 \\
5,270 \\
5,245 \\
5,230\end{array}$ & $\begin{array}{l}17,060 \\
17,241 \\
17,405 \\
17,563\end{array}$ & $\begin{array}{l}3,640 \\
3,685 \\
3,722 \\
3,775\end{array}$ \\
\hline $\begin{array}{l}\text { Females } \\
1981 \\
1986 \\
1991 \\
1996\end{array}$ & $\begin{array}{l}25,474 \\
25,687 \\
26,067 \\
26,381\end{array}$ & $\begin{array}{l}310 \\
319 \\
342 \\
310\end{array}$ & $\begin{array}{l}1,154 \\
1,231 \\
1,328 \\
1,300\end{array}$ & $\begin{array}{l}3,446 \\
3,032 \\
3,050 \\
3,243\end{array}$ & $\begin{array}{l}3,863 \\
3,978 \\
3,527 \\
3,134\end{array}$ & $\begin{array}{l}3,517 \\
3,509 \\
3,943 \\
4,056\end{array}$ & $\begin{array}{l}2,972 \\
3,418 \\
3,517 \\
3,528\end{array}$ & $\begin{array}{l}4,255 \\
4,083 \\
4,208 \\
4,704\end{array}$ & $\begin{array}{l}1,380 \\
1,422 \\
1,319 \\
1,252\end{array}$ & $\begin{array}{l}2,599 \\
2,498 \\
2,479 \\
2,437\end{array}$ & $\begin{array}{l}1,564 \\
1,704 \\
1,761 \\
1,734\end{array}$ & $\begin{array}{l}289 \\
346 \\
411 \\
457\end{array}$ & $\begin{array}{l}126 \\
148 \\
181 \\
227\end{array}$ & $\begin{array}{l}5,309 \\
4,953 \\
5,007 \\
5,168\end{array}$ & $\begin{array}{l}14,207 \\
14,616 \\
14,908 \\
15,106\end{array}$ & $\begin{array}{l}5,958 \\
6,118 \\
6,152 \\
6,107\end{array}$ \\
\hline $\begin{array}{l}2000 \\
2001 \\
2002 \\
2003\end{array}$ & $\begin{array}{l}26,702 \\
26,786 \\
26,868 \\
26,956\end{array}$ & $\begin{array}{l}296 \\
288 \\
287 \\
295\end{array}$ & $\begin{array}{l}1,241 \\
1,220 \\
1,195 \\
1,175\end{array}$ & $\begin{array}{l}3,296 \\
3,287 \\
3,280 \\
3,256\end{array}$ & $\begin{array}{l}3,103 \\
3,156 \\
3,207 \\
3,280\end{array}$ & $\begin{array}{l}3,859 \\
3,778 \\
3,685 \\
3,610\end{array}$ & $\begin{array}{l}3,859 \\
3,935 \\
4,007 \\
4,051\end{array}$ & $\begin{array}{l}4,923 \\
4,992 \\
5,060 \\
5,103\end{array}$ & $\begin{array}{l}1,304 \\
1,297 \\
1,302 \\
1,329\end{array}$ & $\begin{array}{l}2,332 \\
2,326 \\
2,328 \\
2,338\end{array}$ & $\begin{array}{l}1,758 \\
1,771 \\
1,787 \\
1,807\end{array}$ & $\begin{array}{l}476 \\
471 \\
460 \\
436\end{array}$ & $\begin{array}{l}255 \\
263 \\
270 \\
275\end{array}$ & $\begin{array}{l}5,155 \\
5,119 \\
5,091 \\
5,064\end{array}$ & $\begin{array}{l}15,421 \\
15,538 \\
15,631 \\
15,705\end{array}$ & $\begin{array}{l}6,126 \\
6,129 \\
6,146 \\
6,186\end{array}$ \\
\hline $\begin{array}{l}2004 \\
2005 \\
2006 \\
2007\end{array}$ & $\begin{array}{l}27,062 \\
27,223 \\
27,358 \\
27,503\end{array}$ & $\begin{array}{l}306 \\
312 \\
319 \\
329\end{array}$ & $\begin{array}{l}1,165 \\
1,178 \\
1,201 \\
1,233\end{array}$ & $\begin{array}{l}3,224 \\
3,180 \\
3,127 \\
3,082\end{array}$ & $\begin{array}{l}3,342 \\
3,409 \\
3,458 \\
3,505\end{array}$ & $\begin{array}{l}3,552 \\
3,548 \\
3,523 \\
3,490\end{array}$ & $\begin{array}{l}4,097 \\
4,121 \\
4,134 \\
4,132\end{array}$ & $\begin{array}{l}5,141 \\
5,183 \\
5,239 \\
5,237\end{array}$ & $\begin{array}{l}1,365 \\
1,406 \\
1,466 \\
1,581\end{array}$ & $\begin{array}{l}2,345 \\
2,348 \\
2,333 \\
2,342\end{array}$ & $\begin{array}{l}1,815 \\
1,796 \\
1,778 \\
1,767\end{array}$ & $\begin{array}{l}430 \\
456 \\
492 \\
520\end{array}$ & $\begin{array}{l}280 \\
285 \\
286 \\
286\end{array}$ & $\begin{array}{l}5,031 \\
5,008 \\
4,990 \\
4,982\end{array}$ & $\begin{array}{l}15,796 \\
15,922 \\
16,012 \\
16,026\end{array}$ & $\begin{array}{l}6,235 \\
6,292 \\
6,355 \\
6,496\end{array}$ \\
\hline
\end{tabular}

1 Between 2010 and 2020, state pension age will change from 65 years for men and 60 years for women to 65 years for both sexes.

Tel no. for all enquiries relating to population estimates:- 01329444661 


\begin{tabular}{|c|c|c|c|c|c|c|c|c|c|c|c|c|c|c|c|c|}
\hline $\begin{array}{l}\text { Table } 1.4 \\
\text { continued }\end{array}$ & \multicolumn{16}{|c|}{ Population: age and sex } \\
\hline \multicolumn{15}{|c|}{ Constituent countries of the United Kingdom } & \multicolumn{2}{|c|}{ Numbers (thousands } \\
\hline & & \multicolumn{15}{|c|}{ Age group } \\
\hline Mid-year & All ages & Under 1 & $1-4$ & $5-14$ & $15-24$ & $25-34$ & $35-44$ & $45-59$ & $60-64$ & $65-74$ & $75-84$ & $85-89$ & $\begin{array}{c}90 \text { and } \\
\text { over }\end{array}$ & \begin{tabular}{|c|} 
Under \\
16 \\
\end{tabular} & \begin{tabular}{|c|}
$16-$ \\
$64 \mathrm{M} / 59 \mathrm{~F}^{1}$
\end{tabular} \mid & $\begin{array}{l}65 \mathrm{M} / 65 \mathrm{~F}^{1} \\
\text { and over }\end{array}$ \\
\hline $\begin{array}{l}\text { England } \\
\text { Persons } \\
1981 \\
1986 \\
1991 \\
1996\end{array}$ & $\begin{array}{l}46,821 \\
47,188 \\
47,875 \\
48,519\end{array}$ & $\begin{array}{l}598 \\
618 \\
660 \\
603\end{array}$ & $\begin{array}{l}2,235 \\
2,380 \\
2,560 \\
2,523\end{array}$ & $\begin{array}{l}6,678 \\
5,869 \\
5,885 \\
6,255\end{array}$ & $\begin{array}{l}7,440 \\
7,623 \\
6,772 \\
5,985\end{array}$ & $\begin{array}{l}6,703 \\
6,682 \\
7,460 \\
7,667\end{array}$ & $\begin{array}{l}5,663 \\
6,478 \\
6,633 \\
6,638\end{array}$ & $\begin{array}{l}7,948 \\
7,672 \\
7,920 \\
8,822\end{array}$ & $\begin{array}{l}2,449 \\
2,559 \\
2,399 \\
2,310\end{array}$ & $\begin{array}{l}4,347 \\
4,199 \\
4,222 \\
4,217\end{array}$ & $\begin{array}{l}2,249 \\
2,501 \\
2,626 \\
2,631\end{array}$ & $\begin{array}{l}362 \\
435 \\
529 \\
602\end{array}$ & $\begin{array}{l}149 \\
172 \\
210 \\
269\end{array}$ & $\begin{array}{r}10,285 \\
9,583 \\
9,658 \\
9,985\end{array}$ & $\begin{array}{l}28,133 \\
28,962 \\
29,390 \\
29,639\end{array}$ & $\begin{array}{l}8,403 \\
8,643 \\
8,827 \\
8,895\end{array}$ \\
\hline $\begin{array}{l}2000 \\
2001 \\
2002 \\
2003\end{array}$ & $\begin{array}{l}49,233 \\
49,450 \\
49,652 \\
49,866\end{array}$ & $\begin{array}{l}575 \\
558 \\
559 \\
576\end{array}$ & $\begin{array}{l}2,406 \\
2,366 \\
2,313 \\
2,275\end{array}$ & $\begin{array}{l}6,375 \\
6,359 \\
6,348 \\
6,305\end{array}$ & $\begin{array}{l}5,923 \\
6,032 \\
6,153 \\
6,304\end{array}$ & $\begin{array}{l}7,304 \\
7,171 \\
7,003 \\
6,859\end{array}$ & $\begin{array}{l}7,257 \\
7,407 \\
7,550 \\
7,641\end{array}$ & $\begin{array}{l}9,199 \\
9,327 \\
9,439 \\
9,522\end{array}$ & $\begin{array}{l}2,411 \\
2,395 \\
2,399 \\
2,445\end{array}$ & $\begin{array}{l}4,107 \\
4,113 \\
4,129 \\
4,155\end{array}$ & $\begin{array}{l}2,727 \\
2,764 \\
2,803 \\
2,850\end{array}$ & $\begin{array}{l}641 \\
638 \\
625 \\
596\end{array}$ & $\begin{array}{l}309 \\
321 \\
331 \\
340\end{array}$ & $\begin{array}{l}9,980 \\
9,908 \\
9,855 \\
9,812\end{array}$ & $\begin{array}{l}30,243 \\
30,487 \\
30,686 \\
30,867\end{array}$ & $\begin{array}{l}9,010 \\
9,055 \\
9,111 \\
9,188\end{array}$ \\
\hline $\begin{array}{l}2004 \\
2005 \\
2006 \\
2007\end{array}$ & $\begin{array}{l}50,111 \\
50,466 \\
50,763 \\
51,092\end{array}$ & $\begin{array}{l}597 \\
606 \\
620 \\
641\end{array}$ & $\begin{array}{l}2,262 \\
2,289 \\
2,335 \\
2,398\end{array}$ & $\begin{array}{l}6,245 \\
6,161 \\
6,051 \\
5,961\end{array}$ & $\begin{array}{l}6,450 \\
6,583 \\
6,696 \\
6,812\end{array}$ & $\begin{array}{l}6,751 \\
6,742 \\
6,708 \\
6,669\end{array}$ & $\begin{array}{l}7,712 \\
7,772 \\
7,793 \\
7,791\end{array}$ & $\begin{array}{l}9,591 \\
9,675 \\
9,777 \\
9,758\end{array}$ & $\begin{array}{l}2,509 \\
2,586 \\
2,697 \\
2,904\end{array}$ & $\begin{array}{l}4,175 \\
4,189 \\
4,171 \\
4,192\end{array}$ & $\begin{array}{l}2,875 \\
2,865 \\
2,860 \\
2,865\end{array}$ & $\begin{array}{l}593 \\
638 \\
695 \\
739\end{array}$ & $\begin{array}{l}349 \\
357 \\
360 \\
363\end{array}$ & $\begin{array}{l}9,755 \\
9,713 \\
9,674 \\
9,656\end{array}$ & $\begin{array}{l}31,083 \\
31,384 \\
31,627 \\
31,792\end{array}$ & $\begin{array}{l}9,273 \\
9,370 \\
9,462 \\
9,645\end{array}$ \\
\hline $\begin{array}{l}\text { Males } \\
1981 \\
1986 \\
1991 \\
1996\end{array}$ & $\begin{array}{l}22,795 \\
22,949 \\
23,291 \\
23,629\end{array}$ & $\begin{array}{l}306 \\
317 \\
336 \\
309\end{array}$ & $\begin{array}{l}1,147 \\
1,219 \\
1,307 \\
1,294\end{array}$ & $\begin{array}{l}3,430 \\
3,010 \\
3,011 \\
3,198\end{array}$ & $\begin{array}{l}3,790 \\
3,862 \\
3,439 \\
3,023\end{array}$ & $\begin{array}{l}3,377 \\
3,357 \\
3,721 \\
3,818\end{array}$ & $\begin{array}{l}2,856 \\
3,249 \\
3,311 \\
3,302\end{array}$ & $\begin{array}{l}3,938 \\
3,822 \\
3,957 \\
4,390\end{array}$ & $\begin{array}{l}1,154 \\
1,224 \\
1,159 \\
1,133\end{array}$ & $\begin{array}{l}1,902 \\
1,853 \\
1,900 \\
1,932\end{array}$ & $\begin{array}{r}777 \\
897 \\
970 \\
1,003\end{array}$ & $\begin{array}{r}89 \\
108 \\
141 \\
172\end{array}$ & $\begin{array}{l}30 \\
33 \\
39 \\
55\end{array}$ & $\begin{array}{l}5,280 \\
4,911 \\
4,938 \\
5,110\end{array}$ & $\begin{array}{l}14,717 \\
15,147 \\
15,302 \\
15,358\end{array}$ & $\begin{array}{l}2,798 \\
2,891 \\
3,050 \\
3,161\end{array}$ \\
\hline $\begin{array}{l}2000 \\
2001 \\
2002 \\
2003\end{array}$ & $\begin{array}{l}24,030 \\
24,166 \\
24,290 \\
24,419\end{array}$ & $\begin{array}{l}294 \\
285 \\
286 \\
296\end{array}$ & $\begin{array}{l}1,232 \\
1,212 \\
1,182 \\
1,163\end{array}$ & $\begin{array}{l}3,266 \\
3,257 \\
3,253 \\
3,232\end{array}$ & $\begin{array}{l}2,995 \\
3,053 \\
3,127 \\
3,209\end{array}$ & $\begin{array}{l}3,638 \\
3,580 \\
3,500 \\
3,425\end{array}$ & $\begin{array}{l}3,604 \\
3,681 \\
3,755 \\
3,803\end{array}$ & $\begin{array}{l}4,562 \\
4,624 \\
4,673 \\
4,715\end{array}$ & $\begin{array}{l}1,184 \\
1,176 \\
1,176 \\
1,197\end{array}$ & $\begin{array}{l}1,917 \\
1,928 \\
1,942 \\
1,958\end{array}$ & $\begin{array}{l}1,078 \\
1,103 \\
1,128 \\
1,154\end{array}$ & $\begin{array}{l}192 \\
194 \\
193 \\
186\end{array}$ & $\begin{array}{l}69 \\
73 \\
77 \\
80\end{array}$ & $\begin{array}{l}5,113 \\
5,075 \\
5,047 \\
5,028\end{array}$ & $\begin{array}{l}15,661 \\
15,793 \\
15,904 \\
16,012\end{array}$ & $\begin{array}{l}3,256 \\
3,298 \\
3,339 \\
3,379\end{array}$ \\
\hline $\begin{array}{l}2004 \\
2005 \\
2006 \\
2007\end{array}$ & $\begin{array}{l}24,563 \\
24,758 \\
24,926 \\
25,114\end{array}$ & $\begin{array}{l}306 \\
310 \\
317 \\
328\end{array}$ & $\begin{array}{r}1,159 \\
1,172 \\
1,196 \\
1,228\end{array}$ & $\begin{array}{l}3,202 \\
3,160 \\
3,100 \\
3,050\end{array}$ & $\begin{array}{l}3,297 \\
3,365 \\
3,432 \\
3,506\end{array}$ & $\begin{array}{l}3,371 \\
3,365 \\
3,353 \\
3,345\end{array}$ & $\begin{array}{l}3,831 \\
3,868 \\
3,875 \\
3,874\end{array}$ & $\begin{array}{l}4,748 \\
4,791 \\
4,839 \\
4,821\end{array}$ & $\begin{array}{l}1,228 \\
1,267 \\
1,320 \\
1,418\end{array}$ & $\begin{array}{l}1,972 \\
1,984 \\
1,981 \\
1,995\end{array}$ & $\begin{array}{r}1,172 \\
1,179 \\
1,190 \\
1,205\end{array}$ & $\begin{array}{l}190 \\
210 \\
233 \\
251\end{array}$ & $\begin{array}{l}84 \\
88 \\
91 \\
93\end{array}$ & $\begin{array}{l}5,001 \\
4,979 \\
4,957 \\
4,944\end{array}$ & $\begin{array}{l}16,143 \\
16,317 \\
16,475 \\
16,626\end{array}$ & $\begin{array}{l}3,419 \\
3,461 \\
3,494 \\
3,544\end{array}$ \\
\hline $\begin{array}{l}\text { Females } \\
1981 \\
1986 \\
1991 \\
1996\end{array}$ & $\begin{array}{l}24,026 \\
24,239 \\
24,584 \\
24,890\end{array}$ & $\begin{array}{l}292 \\
301 \\
324 \\
293\end{array}$ & $\begin{array}{l}1,088 \\
1,161 \\
1,253 \\
1,229\end{array}$ & $\begin{array}{l}3,248 \\
2,859 \\
2,873 \\
3,056\end{array}$ & $\begin{array}{l}3,650 \\
3,761 \\
3,333 \\
2,961\end{array}$ & $\begin{array}{l}3,327 \\
3,325 \\
3,739 \\
3,849\end{array}$ & $\begin{array}{l}2,807 \\
3,229 \\
3,322 \\
3,336\end{array}$ & $\begin{array}{l}4,009 \\
3,850 \\
3,964 \\
4,432\end{array}$ & $\begin{array}{l}1,295 \\
1,335 \\
1,239 \\
1,177\end{array}$ & $\begin{array}{l}2,445 \\
2,346 \\
2,323 \\
2,286\end{array}$ & $\begin{array}{l}1,472 \\
1,604 \\
1,656 \\
1,628\end{array}$ & $\begin{array}{l}273 \\
326 \\
388 \\
430\end{array}$ & $\begin{array}{l}119 \\
140 \\
171 \\
214\end{array}$ & $\begin{array}{l}5,004 \\
4,672 \\
4,720 \\
4,876\end{array}$ & $\begin{array}{l}13,416 \\
13,815 \\
14,088 \\
14,281\end{array}$ & $\begin{array}{l}5,605 \\
5,752 \\
5,777 \\
5,734\end{array}$ \\
\hline $\begin{array}{l}2000 \\
2001 \\
2002 \\
2003\end{array}$ & $\begin{array}{l}25,203 \\
25,284 \\
25,362 \\
25,448\end{array}$ & $\begin{array}{l}281 \\
273 \\
273 \\
280\end{array}$ & $\begin{array}{l}1,174 \\
1,154 \\
1,131 \\
1,112\end{array}$ & $\begin{array}{l}3,109 \\
3,102 \\
3,095 \\
3,073\end{array}$ & $\begin{array}{l}2,928 \\
2,979 \\
3,026 \\
3,095\end{array}$ & $\begin{array}{l}3,667 \\
3,591 \\
3,503 \\
3,433\end{array}$ & $\begin{array}{l}3,653 \\
3,726 \\
3,795 \\
3,838\end{array}$ & $\begin{array}{l}4,637 \\
4,702 \\
4,767 \\
4,808\end{array}$ & $\begin{array}{l}1,227 \\
1,219 \\
1,223 \\
1,248\end{array}$ & $\begin{array}{l}2,190 \\
2,185 \\
2,187 \\
2,197\end{array}$ & $\begin{array}{l}1,649 \\
1,661 \\
1,676 \\
1,696\end{array}$ & $\begin{array}{l}448 \\
444 \\
433 \\
410\end{array}$ & $\begin{array}{l}240 \\
248 \\
254 \\
260\end{array}$ & $\begin{array}{l}4,867 \\
4,834 \\
4,808 \\
4,784\end{array}$ & $\begin{array}{l}14,582 \\
14,694 \\
14,782 \\
14,854\end{array}$ & $\begin{array}{l}5,755 \\
5,757 \\
5,772 \\
5,809\end{array}$ \\
\hline $\begin{array}{l}2004 \\
2005 \\
2006 \\
2007\end{array}$ & $\begin{array}{l}25,548 \\
25,708 \\
25,837 \\
25,978\end{array}$ & $\begin{array}{l}291 \\
296 \\
303 \\
312\end{array}$ & $\begin{array}{r}1,103 \\
1,117 \\
1,139 \\
1,170\end{array}$ & $\begin{array}{l}3,043 \\
3,001 \\
2,952 \\
2,910\end{array}$ & $\begin{array}{l}3,153 \\
3,218 \\
3,264 \\
3,306\end{array}$ & $\begin{array}{l}3,380 \\
3,378 \\
3,355 \\
3,324\end{array}$ & $\begin{array}{l}3,881 \\
3,905 \\
3,918 \\
3,917\end{array}$ & $\begin{array}{l}4,843 \\
4,885 \\
4,938 \\
4,937\end{array}$ & $\begin{array}{l}1,280 \\
1,319 \\
1,377 \\
1,485\end{array}$ & $\begin{array}{l}2,203 \\
2,206 \\
2,190 \\
2,198\end{array}$ & $\begin{array}{l}1,703 \\
1,686 \\
1,670 \\
1,660\end{array}$ & $\begin{array}{l}403 \\
428 \\
461 \\
488\end{array}$ & $\begin{array}{l}264 \\
269 \\
270 \\
270\end{array}$ & $\begin{array}{l}4,753 \\
4,733 \\
4,717 \\
4,711\end{array}$ & $\begin{array}{l}14,940 \\
15,066 \\
15,152 \\
15,166\end{array}$ & $\begin{array}{l}5,854 \\
5,908 \\
5,968 \\
6,100\end{array}$ \\
\hline $\begin{array}{l}\text { Wales } \\
\text { Persons } \\
1981 \\
1986 \\
1991 \\
1996\end{array}$ & $\begin{array}{l}2,813 \\
2,811 \\
2,873 \\
2,891\end{array}$ & $\begin{array}{l}36 \\
37 \\
38 \\
34\end{array}$ & $\begin{array}{l}136 \\
143 \\
153 \\
146\end{array}$ & $\begin{array}{l}407 \\
357 \\
363 \\
381\end{array}$ & $\begin{array}{l}434 \\
438 \\
393 \\
352\end{array}$ & $\begin{array}{l}383 \\
369 \\
402 \\
409\end{array}$ & $\begin{array}{l}333 \\
378 \\
389 \\
379\end{array}$ & $\begin{array}{l}485 \\
464 \\
486 \\
541\end{array}$ & $\begin{array}{l}158 \\
166 \\
154 \\
147\end{array}$ & $\begin{array}{l}272 \\
271 \\
284 \\
279\end{array}$ & $\begin{array}{l}139 \\
154 \\
164 \\
170\end{array}$ & $\begin{array}{l}21 \\
26 \\
32 \\
37\end{array}$ & $\begin{array}{l}8 \\
10 \\
13 \\
17\end{array}$ & $\begin{array}{l}626 \\
578 \\
589 \\
598\end{array}$ & $\begin{array}{l}1,663 \\
1,686 \\
1,711 \\
1,714\end{array}$ & $\begin{array}{l}525 \\
547 \\
573 \\
578\end{array}$ \\
\hline $\begin{array}{l}2000 \\
2001 \\
2002 \\
2003\end{array}$ & $\begin{array}{l}2,907 \\
2,910 \\
2,920 \\
2,931\end{array}$ & $\begin{array}{l}32 \\
32 \\
30 \\
31\end{array}$ & $\begin{array}{l}138 \\
136 \\
132 \\
129\end{array}$ & $\begin{array}{l}383 \\
382 \\
380 \\
377\end{array}$ & $\begin{array}{l}352 \\
356 \\
3655 \\
376\end{array}$ & $\begin{array}{l}378 \\
365 \\
354 \\
345\end{array}$ & $\begin{array}{l}403 \\
409 \\
414 \\
417\end{array}$ & $\begin{array}{l}565 \\
572 \\
578 \\
582\end{array}$ & $\begin{array}{l}152 \\
154 \\
156 \\
161\end{array}$ & $\begin{array}{l}265 \\
264 \\
265 \\
268\end{array}$ & $\begin{array}{l}180 \\
183 \\
185 \\
187\end{array}$ & $\begin{array}{l}39 \\
39 \\
39 \\
38\end{array}$ & $\begin{array}{l}19 \\
20 \\
20 \\
21\end{array}$ & $\begin{array}{l}591 \\
587 \\
582 \\
577\end{array}$ & $\begin{array}{l}1,734 \\
1,739 \\
1,779 \\
1,759\end{array}$ & $\begin{array}{l}581 \\
584 \\
589 \\
595\end{array}$ \\
\hline $\begin{array}{l}2004 \\
2005 \\
2006 \\
2007\end{array}$ & $\begin{array}{l}2,946 \\
2,954 \\
2,966 \\
2,980\end{array}$ & $\begin{array}{l}32 \\
32 \\
33 \\
34\end{array}$ & $\begin{array}{l}127 \\
126 \\
127 \\
130\end{array}$ & $\begin{array}{l}373 \\
367 \\
361 \\
353\end{array}$ & $\begin{array}{l}385 \\
390 \\
399 \\
407\end{array}$ & $\begin{array}{l}339 \\
335 \\
332 \\
330\end{array}$ & $\begin{array}{l}421 \\
421 \\
421 \\
418\end{array}$ & $\begin{array}{l}586 \\
589 \\
592 \\
590\end{array}$ & $\begin{array}{l}166 \\
171 \\
177 \\
188\end{array}$ & $\begin{array}{l}270 \\
271 \\
273 \\
276\end{array}$ & $\begin{array}{l}188 \\
186 \\
186 \\
185\end{array}$ & $\begin{array}{l}39 \\
42 \\
45 \\
48\end{array}$ & $\begin{array}{l}21 \\
21 \\
22 \\
22\end{array}$ & $\begin{array}{l}572 \\
566 \\
561 \\
557\end{array}$ & $\begin{array}{l}1,773 \\
1,780 \\
1,790 \\
1,797\end{array}$ & $\begin{array}{l}602 \\
608 \\
615 \\
627\end{array}$ \\
\hline $\begin{array}{l}\text { Males } \\
1981 \\
1986 \\
1991 \\
1996\end{array}$ & $\begin{array}{l}1,365 \\
1,362 \\
1,391 \\
1,401\end{array}$ & $\begin{array}{l}18 \\
19 \\
20 \\
17\end{array}$ & $\begin{array}{l}70 \\
73 \\
78 \\
74\end{array}$ & $\begin{array}{l}209 \\
184 \\
186 \\
195\end{array}$ & $\begin{array}{l}221 \\
221 \\
199 \\
179\end{array}$ & $\begin{array}{l}193 \\
186 \\
199 \\
203\end{array}$ & $\begin{array}{l}168 \\
190 \\
194 \\
187\end{array}$ & $\begin{array}{l}240 \\
231 \\
242 \\
269\end{array}$ & $\begin{array}{l}73 \\
79 \\
74 \\
72\end{array}$ & $\begin{array}{l}118 \\
119 \\
128 \\
128\end{array}$ & $\begin{array}{l}48 \\
54 \\
60 \\
64\end{array}$ & $\begin{array}{r}5 \\
7 \\
8 \\
10\end{array}$ & $\begin{array}{l}2 \\
2 \\
2 \\
3\end{array}$ & $\begin{array}{l}321 \\
297 \\
302 \\
306\end{array}$ & $\begin{array}{l}871 \\
885 \\
891 \\
890\end{array}$ & $\begin{array}{l}173 \\
181 \\
198 \\
206\end{array}$ \\
\hline $\begin{array}{l}2000 \\
2001 \\
2002 \\
2003\end{array}$ & $\begin{array}{l}1,408 \\
1,409 \\
1,414 \\
1,423\end{array}$ & $\begin{array}{l}16 \\
16 \\
16 \\
16\end{array}$ & $\begin{array}{l}71 \\
69 \\
68 \\
66\end{array}$ & $\begin{array}{l}196 \\
196 \\
195 \\
194\end{array}$ & $\begin{array}{l}177 \\
179 \\
184 \\
190\end{array}$ & $\begin{array}{l}185 \\
178 \\
172 \\
168\end{array}$ & $\begin{array}{l}198 \\
200 \\
202 \\
204\end{array}$ & $\begin{array}{l}280 \\
283 \\
285 \\
287\end{array}$ & $\begin{array}{l}75 \\
75 \\
77 \\
79\end{array}$ & $\begin{array}{l}124 \\
124 \\
125 \\
127\end{array}$ & $\begin{array}{l}71 \\
73 \\
74 \\
75\end{array}$ & $\begin{array}{l}12 \\
12 \\
12 \\
11\end{array}$ & $\begin{array}{l}4 \\
4 \\
5 \\
5\end{array}$ & $\begin{array}{l}303 \\
301 \\
299 \\
296\end{array}$ & $\begin{array}{l}895 \\
895 \\
900 \\
908\end{array}$ & $\begin{array}{l}210 \\
212 \\
215 \\
218\end{array}$ \\
\hline $\begin{array}{l}2004 \\
2005 \\
2006 \\
2007\end{array}$ & $\begin{array}{l}1,432 \\
1,439 \\
1,445 \\
1,454\end{array}$ & $\begin{array}{l}16 \\
17 \\
17 \\
17\end{array}$ & $\begin{array}{l}65 \\
65 \\
65 \\
67\end{array}$ & $\begin{array}{l}192 \\
189 \\
185 \\
181\end{array}$ & $\begin{array}{l}196 \\
200 \\
204 \\
209\end{array}$ & $\begin{array}{l}166 \\
166 \\
164 \\
164\end{array}$ & $\begin{array}{l}205 \\
205 \\
205 \\
203\end{array}$ & $\begin{array}{l}288 \\
290 \\
291 \\
289\end{array}$ & $\begin{array}{l}82 \\
84 \\
87 \\
93\end{array}$ & $\begin{array}{l}128 \\
129 \\
130 \\
132\end{array}$ & $\begin{array}{l}76 \\
77 \\
77 \\
78\end{array}$ & $\begin{array}{l}12 \\
13 \\
15 \\
16\end{array}$ & $\begin{array}{l}5 \\
5 \\
5 \\
6\end{array}$ & $\begin{array}{l}294 \\
291 \\
288 \\
286\end{array}$ & $\begin{array}{l}917 \\
924 \\
929 \\
937\end{array}$ & $\begin{array}{l}221 \\
224 \\
227 \\
231\end{array}$ \\
\hline $\begin{array}{l}\text { Females } \\
1981 \\
1986 \\
1991 \\
1996\end{array}$ & $\begin{array}{l}1,448 \\
1,449 \\
1,482 \\
1,490\end{array}$ & $\begin{array}{l}18 \\
18 \\
19 \\
16\end{array}$ & $\begin{array}{l}66 \\
70 \\
75 \\
71\end{array}$ & $\begin{array}{l}199 \\
173 \\
177 \\
186\end{array}$ & $\begin{array}{l}213 \\
217 \\
194 \\
173\end{array}$ & $\begin{array}{l}190 \\
184 \\
203 \\
206\end{array}$ & $\begin{array}{l}165 \\
188 \\
195 \\
192\end{array}$ & $\begin{array}{l}246 \\
233 \\
244 \\
272\end{array}$ & $\begin{array}{l}85 \\
87 \\
80 \\
75\end{array}$ & $\begin{array}{l}154 \\
152 \\
156 \\
151\end{array}$ & $\begin{array}{r}91 \\
100 \\
104 \\
106\end{array}$ & $\begin{array}{l}16 \\
20 \\
24 \\
27\end{array}$ & $\begin{array}{r}6 \\
8 \\
10 \\
13\end{array}$ & $\begin{array}{l}305 \\
282 \\
288 \\
293\end{array}$ & $\begin{array}{l}791 \\
801 \\
820 \\
825\end{array}$ & $\begin{array}{l}352 \\
366 \\
375 \\
373\end{array}$ \\
\hline $\begin{array}{l}2000 \\
2001 \\
2002 \\
2003\end{array}$ & $\begin{array}{l}1,499 \\
1,502 \\
1,506 \\
1,508\end{array}$ & $\begin{array}{l}15 \\
15 \\
15 \\
15\end{array}$ & $\begin{array}{l}67 \\
66 \\
65 \\
63\end{array}$ & $\begin{array}{l}186 \\
186 \\
185 \\
183\end{array}$ & $\begin{array}{l}175 \\
177 \\
181 \\
185\end{array}$ & $\begin{array}{l}192 \\
187 \\
182 \\
176\end{array}$ & $\begin{array}{l}206 \\
209 \\
212 \\
214\end{array}$ & $\begin{array}{l}285 \\
289 \\
293 \\
295\end{array}$ & $\begin{array}{l}77 \\
78 \\
80 \\
82\end{array}$ & $\begin{array}{l}142 \\
141 \\
140 \\
141\end{array}$ & $\begin{array}{l}109 \\
110 \\
111 \\
112\end{array}$ & $\begin{array}{l}28 \\
27 \\
27 \\
27\end{array}$ & $\begin{array}{l}15 \\
15 \\
16 \\
16\end{array}$ & $\begin{array}{l}288 \\
286 \\
283 \\
280\end{array}$ & $\begin{array}{l}840 \\
844 \\
849 \\
851\end{array}$ & $\begin{array}{l}371 \\
372 \\
374 \\
377\end{array}$ \\
\hline $\begin{array}{l}2004 \\
2005 \\
2006 \\
2007\end{array}$ & $\begin{array}{l}1,514 \\
1,515 \\
1,521 \\
1,526\end{array}$ & $\begin{array}{l}15 \\
16 \\
16 \\
16\end{array}$ & $\begin{array}{l}62 \\
61 \\
62 \\
63\end{array}$ & $\begin{array}{l}182 \\
179 \\
176 \\
172\end{array}$ & $\begin{array}{l}189 \\
191 \\
195 \\
198\end{array}$ & $\begin{array}{l}172 \\
170 \\
168 \\
166\end{array}$ & $\begin{array}{l}216 \\
216 \\
216 \\
215\end{array}$ & $\begin{array}{l}298 \\
299 \\
301 \\
300\end{array}$ & $\begin{array}{l}84 \\
87 \\
90 \\
96\end{array}$ & $\begin{array}{l}142 \\
142 \\
143 \\
144\end{array}$ & $\begin{array}{l}112 \\
110 \\
108 \\
107\end{array}$ & $\begin{array}{l}26 \\
28 \\
30 \\
32\end{array}$ & $\begin{array}{l}16 \\
16 \\
16 \\
16\end{array}$ & $\begin{array}{l}278 \\
275 \\
273 \\
271\end{array}$ & $\begin{array}{l}856 \\
856 \\
861 \\
860\end{array}$ & $\begin{array}{l}380 \\
383 \\
387 \\
395\end{array}$ \\
\hline
\end{tabular}




\begin{tabular}{|c|c|c|c|c|c|c|c|c|c|c|c|c|c|c|c|c|}
\hline $\begin{array}{l}\text { Table } 1.4 \\
\text { continued }\end{array}$ & \multicolumn{16}{|c|}{ Population: age and sex } \\
\hline \multicolumn{15}{|c|}{ Constituent countries of the United Kingdom } & \multicolumn{2}{|c|}{ Numbers (thousands) } \\
\hline & & \multicolumn{15}{|c|}{ Age group } \\
\hline Mid-year & All ages & Under 1 & $1-4$ & $5-14$ & $15-24$ & $25-34$ & $35-44$ & $45-59$ & $60-64$ & $65-74$ & $75-84$ & $85-89$ & $\begin{array}{c}90 \text { and } \\
\text { over }\end{array}$ & $\begin{array}{c}\text { Under } \\
16\end{array}$ & $\begin{array}{c}16- \\
64 \mathrm{M} / 59 \mathrm{~F}^{1}\end{array}$ & $\begin{array}{l}65 \mathrm{M} / 60 \mathrm{~F}^{1} \\
\text { and over }\end{array}$ \\
\hline $\begin{array}{l}\text { Scotland } \\
\text { Persons } \\
1981 \\
1986 \\
1991 \\
1996\end{array}$ & $\begin{array}{l}5,180 \\
5,112 \\
5,083 \\
5,092\end{array}$ & $\begin{array}{l}69 \\
66 \\
66 \\
59\end{array}$ & $\begin{array}{l}249 \\
257 \\
258 \\
252\end{array}$ & $\begin{array}{l}780 \\
656 \\
634 \\
643\end{array}$ & $\begin{array}{l}875 \\
863 \\
746 \\
651\end{array}$ & $\begin{array}{l}724 \\
739 \\
795 \\
798\end{array}$ & $\begin{array}{l}603 \\
665 \\
696 \\
722\end{array}$ & $\begin{array}{l}880 \\
849 \\
853 \\
925\end{array}$ & $\begin{array}{l}260 \\
273 \\
265 \\
259\end{array}$ & $\begin{array}{l}460 \\
435 \\
441 \\
448\end{array}$ & $\begin{array}{l}232 \\
252 \\
259 \\
256\end{array}$ & $\begin{array}{l}35 \\
42 \\
51 \\
57\end{array}$ & $\begin{array}{l}14 \\
15 \\
19 \\
24\end{array}$ & $\begin{array}{l}1,188 \\
1,061 \\
1,021 \\
1,019\end{array}$ & $\begin{array}{l}3,110 \\
3,161 \\
3,151 \\
3,151\end{array}$ & $\begin{array}{l}882 \\
890 \\
912 \\
922\end{array}$ \\
\hline $\begin{array}{l}2000 \\
2001 \\
2002 \\
2003\end{array}$ & $\begin{array}{l}5,063 \\
5,064 \\
5,055 \\
5,057\end{array}$ & $\begin{array}{l}53 \\
52 \\
51 \\
52\end{array}$ & $\begin{array}{l}230 \\
224 \\
217 \\
212\end{array}$ & $\begin{array}{l}636 \\
629 \\
622 \\
614\end{array}$ & $\begin{array}{l}628 \\
633 \\
639 \\
648\end{array}$ & $\begin{array}{l}717 \\
696 \\
669 \\
648\end{array}$ & $\begin{array}{l}774 \\
782 \\
788 \\
793\end{array}$ & $\begin{array}{r}962 \\
979 \\
993 \\
1,008\end{array}$ & $\begin{array}{l}263 \\
262 \\
262 \\
265\end{array}$ & $\begin{array}{l}445 \\
447 \\
449 \\
452\end{array}$ & $\begin{array}{l}267 \\
272 \\
276 \\
281\end{array}$ & $\begin{array}{l}59 \\
59 \\
58 \\
55\end{array}$ & $\begin{array}{l}28 \\
29 \\
30 \\
31\end{array}$ & $\begin{array}{l}985 \\
970 \\
955 \\
943\end{array}$ & $\begin{array}{l}3,141 \\
3,150 \\
3,150 \\
3,156\end{array}$ & $\begin{array}{l}937 \\
944 \\
950 \\
958\end{array}$ \\
\hline $\begin{array}{l}2004 \\
2005 \\
2006 \\
2007\end{array}$ & $\begin{array}{l}5,078 \\
5,095 \\
5,117 \\
5,144\end{array}$ & $\begin{array}{l}54 \\
54 \\
55 \\
57\end{array}$ & $\begin{array}{l}210 \\
211 \\
213 \\
218\end{array}$ & $\begin{array}{l}609 \\
600 \\
588 \\
576\end{array}$ & $\begin{array}{l}653 \\
659 \\
668 \\
676\end{array}$ & $\begin{array}{l}635 \\
629 \\
627 \\
629\end{array}$ & $\begin{array}{l}796 \\
794 \\
790 \\
781\end{array}$ & $\begin{array}{l}1,025 \\
1,042 \\
1,058 \\
1,060\end{array}$ & $\begin{array}{l}270 \\
273 \\
280 \\
301\end{array}$ & $\begin{array}{l}455 \\
457 \\
456 \\
457\end{array}$ & $\begin{array}{l}286 \\
286 \\
287 \\
290\end{array}$ & $\begin{array}{l}54 \\
59 \\
63 \\
66\end{array}$ & $\begin{array}{l}31 \\
32 \\
32 \\
32\end{array}$ & $\begin{array}{l}935 \\
929 \\
922 \\
917\end{array}$ & $\begin{array}{l}3,175 \\
3,191 \\
3,213 \\
3,227\end{array}$ & $\begin{array}{r}968 \\
975 \\
983 \\
1,001\end{array}$ \\
\hline $\begin{array}{l}\text { Males } \\
1981 \\
1986 \\
1991 \\
1996\end{array}$ & $\begin{array}{l}2,495 \\
2,462 \\
2,445 \\
2,447\end{array}$ & $\begin{array}{l}35 \\
34 \\
34 \\
30\end{array}$ & $\begin{array}{l}128 \\
131 \\
132 \\
128\end{array}$ & $\begin{array}{l}400 \\
336 \\
324 \\
328\end{array}$ & $\begin{array}{l}445 \\
438 \\
377 \\
327\end{array}$ & $\begin{array}{l}364 \\
371 \\
394 \\
392\end{array}$ & $\begin{array}{l}298 \\
331 \\
345 \\
355\end{array}$ & $\begin{array}{l}424 \\
410 \\
415 \\
454\end{array}$ & $\begin{array}{l}118 \\
127 \\
124 \\
122\end{array}$ & $\begin{array}{l}194 \\
184 \\
192 \\
198\end{array}$ & $\begin{array}{l}77 \\
86 \\
91 \\
93\end{array}$ & $\begin{array}{r}8 \\
10 \\
13 \\
15\end{array}$ & $\begin{array}{l}3 \\
3 \\
3 \\
5\end{array}$ & $\begin{array}{l}610 \\
543 \\
522 \\
521\end{array}$ & $\begin{array}{l}1,603 \\
1,636 \\
1,623 \\
1,616\end{array}$ & $\begin{array}{l}282 \\
283 \\
299 \\
310\end{array}$ \\
\hline $\begin{array}{l}2000 \\
2001 \\
2002 \\
2003\end{array}$ & $\begin{array}{l}2,432 \\
2,434 \\
2,432 \\
2,435\end{array}$ & $\begin{array}{l}28 \\
26 \\
26 \\
26\end{array}$ & $\begin{array}{l}118 \\
115 \\
111 \\
108\end{array}$ & $\begin{array}{l}326 \\
322 \\
319 \\
314\end{array}$ & $\begin{array}{l}315 \\
319 \\
324 \\
329\end{array}$ & $\begin{array}{l}347 \\
337 \\
325 \\
315\end{array}$ & $\begin{array}{l}377 \\
379 \\
382 \\
383\end{array}$ & $\begin{array}{l}474 \\
483 \\
490 \\
496\end{array}$ & $\begin{array}{l}125 \\
125 \\
125 \\
126\end{array}$ & $\begin{array}{l}199 \\
200 \\
202 \\
204\end{array}$ & $\begin{array}{l}100 \\
103 \\
106 \\
108\end{array}$ & $\begin{array}{l}17 \\
17 \\
17 \\
16\end{array}$ & $\begin{array}{l}6 \\
6 \\
7 \\
7\end{array}$ & $\begin{array}{l}505 \\
497 \\
489 \\
483\end{array}$ & $\begin{array}{l}1,606 \\
1,610 \\
1,612 \\
1,616\end{array}$ & $\begin{array}{l}322 \\
327 \\
331 \\
336\end{array}$ \\
\hline $\begin{array}{l}2004 \\
2005 \\
2006 \\
2007\end{array}$ & $\begin{array}{l}2,446 \\
2,456 \\
2,469 \\
2,486\end{array}$ & $\begin{array}{l}28 \\
28 \\
28 \\
29\end{array}$ & $\begin{array}{l}107 \\
107 \\
109 \\
112\end{array}$ & $\begin{array}{l}312 \\
307 \\
301 \\
295\end{array}$ & $\begin{array}{l}332 \\
335 \\
340 \\
345\end{array}$ & $\begin{array}{l}310 \\
309 \\
310 \\
313\end{array}$ & $\begin{array}{l}384 \\
382 \\
380 \\
375\end{array}$ & $\begin{array}{l}503 \\
511 \\
517 \\
517\end{array}$ & $\begin{array}{l}129 \\
131 \\
135 \\
146\end{array}$ & $\begin{array}{l}207 \\
208 \\
208 \\
210\end{array}$ & $\begin{array}{l}111 \\
112 \\
113 \\
116\end{array}$ & $\begin{array}{l}16 \\
18 \\
20 \\
21\end{array}$ & $\begin{array}{l}7 \\
7 \\
8 \\
8\end{array}$ & $\begin{array}{l}479 \\
476 \\
472 \\
469\end{array}$ & $\begin{array}{l}1,627 \\
1,635 \\
1,649 \\
1,662\end{array}$ & $\begin{array}{l}341 \\
345 \\
349 \\
354\end{array}$ \\
\hline $\begin{array}{l}\text { Females } \\
1981 \\
1986 \\
1991 \\
1996\end{array}$ & $\begin{array}{r}2,685 \\
2,649 \\
2,639 \\
2,645\end{array}$ & $\begin{array}{l}33 \\
32 \\
32 \\
28\end{array}$ & $\begin{array}{l}121 \\
126 \\
126 \\
123\end{array}$ & $\begin{array}{l}380 \\
320 \\
309 \\
315\end{array}$ & $\begin{array}{l}430 \\
424 \\
369 \\
324\end{array}$ & $\begin{array}{l}359 \\
368 \\
402 \\
406\end{array}$ & $\begin{array}{l}305 \\
334 \\
351 \\
367\end{array}$ & $\begin{array}{l}456 \\
439 \\
437 \\
470\end{array}$ & $\begin{array}{l}142 \\
146 \\
141 \\
137\end{array}$ & $\begin{array}{l}265 \\
250 \\
249 \\
250\end{array}$ & $\begin{array}{l}155 \\
166 \\
168 \\
164\end{array}$ & $\begin{array}{l}27 \\
32 \\
38 \\
42\end{array}$ & $\begin{array}{l}11 \\
12 \\
16 \\
20\end{array}$ & $\begin{array}{l}579 \\
518 \\
499 \\
498\end{array}$ & $\begin{array}{l}1,506 \\
1,525 \\
1,528 \\
1,535\end{array}$ & $\begin{array}{l}600 \\
606 \\
612 \\
612\end{array}$ \\
\hline $\begin{array}{l}2000 \\
2001 \\
2002 \\
2003\end{array}$ & $\begin{array}{l}2,631 \\
2,630 \\
2,623 \\
2,623\end{array}$ & $\begin{array}{l}26 \\
26 \\
25 \\
25\end{array}$ & $\begin{array}{l}112 \\
109 \\
106 \\
104\end{array}$ & $\begin{array}{l}310 \\
307 \\
303 \\
300\end{array}$ & $\begin{array}{l}313 \\
314 \\
315 \\
318\end{array}$ & $\begin{array}{l}369 \\
359 \\
344 \\
332\end{array}$ & $\begin{array}{l}397 \\
403 \\
406 \\
410\end{array}$ & $\begin{array}{l}488 \\
496 \\
504 \\
512\end{array}$ & $\begin{array}{l}138 \\
137 \\
137 \\
139\end{array}$ & $\begin{array}{l}246 \\
246 \\
247 \\
248\end{array}$ & $\begin{array}{l}166 \\
169 \\
171 \\
173\end{array}$ & $\begin{array}{l}43 \\
43 \\
41 \\
39\end{array}$ & $\begin{array}{l}22 \\
23 \\
23 \\
24\end{array}$ & $\begin{array}{l}480 \\
473 \\
466 \\
460\end{array}$ & $\begin{array}{l}1,535 \\
1,540 \\
1,538 \\
1,540\end{array}$ & $\begin{array}{l}616 \\
617 \\
619 \\
622\end{array}$ \\
\hline $\begin{array}{l}2004 \\
2005 \\
2006 \\
2007\end{array}$ & $\begin{array}{l}2,632 \\
2,639 \\
2,647 \\
2,659\end{array}$ & $\begin{array}{l}26 \\
26 \\
27 \\
28\end{array}$ & $\begin{array}{l}103 \\
103 \\
104 \\
106\end{array}$ & $\begin{array}{l}297 \\
293 \\
287 \\
281\end{array}$ & $\begin{array}{l}321 \\
324 \\
328 \\
332\end{array}$ & $\begin{array}{l}325 \\
320 \\
317 \\
316\end{array}$ & $\begin{array}{l}412 \\
411 \\
410 \\
406\end{array}$ & $\begin{array}{l}521 \\
531 \\
541 \\
542\end{array}$ & $\begin{array}{l}141 \\
142 \\
145 \\
155\end{array}$ & $\begin{array}{l}248 \\
249 \\
247 \\
247\end{array}$ & $\begin{array}{l}175 \\
174 \\
174 \\
174\end{array}$ & $\begin{array}{l}38 \\
41 \\
43 \\
45\end{array}$ & $\begin{array}{l}24 \\
25 \\
25 \\
24\end{array}$ & $\begin{array}{l}457 \\
453 \\
450 \\
448\end{array}$ & $\begin{array}{r}1,549 \\
1,556 \\
1,564 \\
1,564\end{array}$ & $\begin{array}{l}627 \\
630 \\
634 \\
646\end{array}$ \\
\hline $\begin{array}{l}\text { Northern Irela } \\
\text { Persons } \\
1981 \\
1986 \\
1991 \\
1996\end{array}$ & $\begin{array}{l}1,543 \\
1,574 \\
1,607 \\
1,662\end{array}$ & $\begin{array}{l}27 \\
28 \\
26 \\
24\end{array}$ & $\begin{array}{r}106 \\
107 \\
106 \\
99\end{array}$ & $\begin{array}{l}282 \\
261 \\
260 \\
266\end{array}$ & $\begin{array}{l}271 \\
277 \\
256 \\
244\end{array}$ & $\begin{array}{l}200 \\
217 \\
240 \\
257\end{array}$ & $\begin{array}{l}175 \\
190 \\
200 \\
220\end{array}$ & $\begin{array}{l}227 \\
227 \\
241 \\
266\end{array}$ & $\begin{array}{l}68 \\
71 \\
70 \\
70\end{array}$ & $\begin{array}{l}116 \\
115 \\
121 \\
123\end{array}$ & $\begin{array}{l}57 \\
64 \\
69 \\
72\end{array}$ & $\begin{array}{l}16 \\
14 \\
15\end{array}$ & $\begin{array}{l}\ddot{0} \\
\ddot{6} \\
7\end{array}$ & $\begin{array}{l}444 \\
423 \\
417 \\
415\end{array}$ & $\begin{array}{l}874 \\
917 \\
945 \\
993\end{array}$ & $\begin{array}{l}224 \\
234 \\
246 \\
253\end{array}$ \\
\hline $\begin{array}{l}2000 \\
2001 \\
2002 \\
2003\end{array}$ & $\begin{array}{l}1,683 \\
1,689 \\
1,697 \\
1,703\end{array}$ & $\begin{array}{l}22 \\
22 \\
22 \\
21\end{array}$ & $\begin{array}{l}95 \\
93 \\
91 \\
89\end{array}$ & $\begin{array}{l}259 \\
255 \\
253 \\
251\end{array}$ & $\begin{array}{l}237 \\
240 \\
243 \\
246\end{array}$ & $\begin{array}{l}247 \\
243 \\
238 \\
233\end{array}$ & $\begin{array}{l}243 \\
248 \\
251 \\
254\end{array}$ & $\begin{array}{l}284 \\
290 \\
296 \\
301\end{array}$ & $\begin{array}{l}73 \\
74 \\
75 \\
78\end{array}$ & $\begin{array}{l}123 \\
123 \\
125 \\
126\end{array}$ & $\begin{array}{l}75 \\
77 \\
79 \\
81\end{array}$ & $\begin{array}{l}16 \\
16 \\
16 \\
16\end{array}$ & $\begin{array}{l}7 \\
7 \\
7 \\
8\end{array}$ & $\begin{array}{l}403 \\
397 \\
393 \\
388\end{array}$ & $\begin{array}{l}1,020 \\
1,030 \\
1,037 \\
1,044\end{array}$ & $\begin{array}{l}259 \\
262 \\
266 \\
271\end{array}$ \\
\hline $\begin{array}{l}2004 \\
2005 \\
2006 \\
2007\end{array}$ & $\begin{array}{l}1,710 \\
1,724 \\
1,742 \\
1,759\end{array}$ & $\begin{array}{l}22 \\
23 \\
23 \\
24\end{array}$ & $\begin{array}{l}87 \\
88 \\
89 \\
91\end{array}$ & $\begin{array}{l}248 \\
245 \\
242 \\
239\end{array}$ & $\begin{array}{l}250 \\
253 \\
258 \\
260\end{array}$ & $\begin{array}{l}229 \\
228 \\
229 \\
231\end{array}$ & $\begin{array}{l}256 \\
257 \\
259 \\
259\end{array}$ & $\begin{array}{l}305 \\
310 \\
316 \\
321\end{array}$ & $\begin{array}{l}81 \\
84 \\
87 \\
90\end{array}$ & $\begin{array}{l}127 \\
128 \\
130 \\
132\end{array}$ & $\begin{array}{l}82 \\
83 \\
83 \\
84\end{array}$ & $\begin{array}{l}16 \\
17 \\
18 \\
19\end{array}$ & $\begin{array}{l}8 \\
8 \\
8 \\
8\end{array}$ & $\begin{array}{l}383 \\
381 \\
380 \\
380\end{array}$ & $\begin{array}{l}1,052 \\
1,064 \\
1,077 \\
1,089\end{array}$ & $\begin{array}{l}275 \\
280 \\
284 \\
290\end{array}$ \\
\hline $\begin{array}{l}\text { Males } \\
1981 \\
1986 \\
1991 \\
1996\end{array}$ & $\begin{array}{l}757 \\
768 \\
783 \\
810\end{array}$ & $\begin{array}{l}14 \\
14 \\
13 \\
12\end{array}$ & $\begin{array}{l}54 \\
55 \\
54 \\
51\end{array}$ & $\begin{array}{l}145 \\
134 \\
133 \\
136\end{array}$ & $\begin{array}{l}140 \\
142 \\
131 \\
124\end{array}$ & $\begin{array}{l}102 \\
109 \\
119 \\
128\end{array}$ & $\begin{array}{r}87 \\
95 \\
100 \\
109\end{array}$ & $\begin{array}{l}109 \\
110 \\
118 \\
131\end{array}$ & $\begin{array}{l}32 \\
33 \\
32 \\
33\end{array}$ & $\begin{array}{l}50 \\
50 \\
53 \\
54\end{array}$ & $\begin{array}{l}21 \\
23 \\
26 \\
27\end{array}$ & $\begin{array}{l}\ddot{4} \\
4 \\
4\end{array}$ & $\begin{array}{l}\ddot{0} \\
\ddot{1} \\
1\end{array}$ & $\begin{array}{l}228 \\
217 \\
213 \\
212\end{array}$ & $\begin{array}{l}454 \\
474 \\
487 \\
511\end{array}$ & $\begin{array}{l}75 \\
77 \\
83 \\
87\end{array}$ \\
\hline $\begin{array}{l}2000 \\
2001 \\
2002 \\
2003\end{array}$ & $\begin{array}{l}820 \\
824 \\
829 \\
833\end{array}$ & $\begin{array}{l}11 \\
11 \\
11 \\
11\end{array}$ & $\begin{array}{l}49 \\
48 \\
47 \\
46\end{array}$ & $\begin{array}{l}133 \\
131 \\
130 \\
129\end{array}$ & $\begin{array}{l}120 \\
122 \\
124 \\
126\end{array}$ & $\begin{array}{l}122 \\
120 \\
117 \\
115\end{array}$ & $\begin{array}{l}119 \\
122 \\
123 \\
124\end{array}$ & $\begin{array}{l}141 \\
144 \\
147 \\
149\end{array}$ & $\begin{array}{l}35 \\
35 \\
36 \\
38\end{array}$ & $\begin{array}{l}55 \\
56 \\
56 \\
57\end{array}$ & $\begin{array}{l}29 \\
30 \\
31 \\
31\end{array}$ & $\begin{array}{l}5 \\
5 \\
5 \\
5\end{array}$ & $\begin{array}{l}2 \\
2 \\
2 \\
2\end{array}$ & $\begin{array}{l}207 \\
204 \\
202 \\
199\end{array}$ & $\begin{array}{l}524 \\
529 \\
534 \\
538\end{array}$ & $\begin{array}{l}90 \\
92 \\
94 \\
95\end{array}$ \\
\hline $\begin{array}{l}2004 \\
2005 \\
2006 \\
2007\end{array}$ & $\begin{array}{l}836 \\
844 \\
853 \\
862\end{array}$ & $\begin{array}{l}11 \\
12 \\
12 \\
13\end{array}$ & $\begin{array}{l}45 \\
45 \\
46 \\
47\end{array}$ & $\begin{array}{l}127 \\
126 \\
124 \\
123\end{array}$ & $\begin{array}{l}128 \\
130 \\
132 \\
134\end{array}$ & $\begin{array}{l}113 \\
113 \\
113 \\
114\end{array}$ & $\begin{array}{l}125 \\
126 \\
127 \\
127\end{array}$ & $\begin{array}{l}151 \\
153 \\
156 \\
158\end{array}$ & $\begin{array}{l}39 \\
41 \\
42 \\
44\end{array}$ & $\begin{array}{l}58 \\
59 \\
60 \\
61\end{array}$ & $\begin{array}{l}32 \\
32 \\
33 \\
33\end{array}$ & $\begin{array}{l}5 \\
5 \\
6 \\
6\end{array}$ & $\begin{array}{l}2 \\
2 \\
2 \\
2\end{array}$ & $\begin{array}{l}197 \\
196 \\
195 \\
195\end{array}$ & $\begin{array}{l}542 \\
550 \\
558 \\
564\end{array}$ & $\begin{array}{r}97 \\
99 \\
101 \\
103\end{array}$ \\
\hline $\begin{array}{l}\text { Females } \\
1981 \\
1986 \\
1991 \\
1996\end{array}$ & $\begin{array}{l}786 \\
805 \\
824 \\
851\end{array}$ & $\begin{array}{l}13 \\
13 \\
13 \\
11\end{array}$ & $\begin{array}{l}52 \\
52 \\
52 \\
49\end{array}$ & $\begin{array}{l}137 \\
127 \\
127 \\
130\end{array}$ & $\begin{array}{l}130 \\
135 \\
125 \\
120\end{array}$ & $\begin{array}{r}98 \\
107 \\
121 \\
129\end{array}$ & $\begin{array}{r}88 \\
96 \\
100 \\
110\end{array}$ & $\begin{array}{l}118 \\
118 \\
123 \\
135\end{array}$ & $\begin{array}{l}37 \\
38 \\
38 \\
37\end{array}$ & $\begin{array}{l}66 \\
65 \\
67 \\
69\end{array}$ & $\begin{array}{l}37 \\
41 \\
44 \\
45\end{array}$ & $\begin{array}{l}12 \\
10 \\
11\end{array}$ & $\begin{array}{l}\ddot{4} \\
6\end{array}$ & $\begin{array}{l}216 \\
206 \\
203 \\
203\end{array}$ & $\begin{array}{l}420 \\
442 \\
458 \\
482\end{array}$ & $\begin{array}{l}150 \\
157 \\
163 \\
167\end{array}$ \\
\hline $\begin{array}{l}2000 \\
2001 \\
2002 \\
2003\end{array}$ & $\begin{array}{l}862 \\
865 \\
868 \\
870\end{array}$ & $\begin{array}{l}11 \\
10 \\
11 \\
10\end{array}$ & $\begin{array}{l}46 \\
45 \\
44 \\
43\end{array}$ & $\begin{array}{l}126 \\
124 \\
123 \\
122\end{array}$ & $\begin{array}{l}118 \\
119 \\
119 \\
120\end{array}$ & $\begin{array}{l}125 \\
123 \\
120 \\
118\end{array}$ & $\begin{array}{l}124 \\
126 \\
128 \\
129\end{array}$ & $\begin{array}{l}143 \\
146 \\
149 \\
152\end{array}$ & $\begin{array}{l}38 \\
38 \\
39 \\
40\end{array}$ & $\begin{array}{l}68 \\
68 \\
68 \\
68\end{array}$ & $\begin{array}{l}46 \\
47 \\
48 \\
49\end{array}$ & $\begin{array}{l}11 \\
11 \\
11 \\
11\end{array}$ & $\begin{array}{l}6 \\
6 \\
6 \\
6\end{array}$ & $\begin{array}{l}196 \\
193 \\
191 \\
189\end{array}$ & $\begin{array}{l}497 \\
501 \\
504 \\
506\end{array}$ & $\begin{array}{l}169 \\
170 \\
173 \\
175\end{array}$ \\
\hline $\begin{array}{l}2004 \\
2005 \\
2006 \\
2007\end{array}$ & $\begin{array}{l}874 \\
880 \\
888 \\
897\end{array}$ & $\begin{array}{l}11 \\
11 \\
11 \\
12\end{array}$ & $\begin{array}{l}42 \\
43 \\
43 \\
44\end{array}$ & $\begin{array}{l}121 \\
119 \\
118 \\
116\end{array}$ & $\begin{array}{l}122 \\
123 \\
126 \\
127\end{array}$ & $\begin{array}{l}116 \\
115 \\
115 \\
117\end{array}$ & $\begin{array}{l}130 \\
131 \\
132 \\
132\end{array}$ & $\begin{array}{l}154 \\
157 \\
160 \\
163\end{array}$ & $\begin{array}{l}42 \\
43 \\
45 \\
46\end{array}$ & $\begin{array}{l}69 \\
69 \\
69 \\
70\end{array}$ & $\begin{array}{l}50 \\
50 \\
51 \\
51\end{array}$ & $\begin{array}{l}11 \\
11 \\
12 \\
13\end{array}$ & $\begin{array}{l}6 \\
6 \\
6 \\
6\end{array}$ & $\begin{array}{l}187 \\
186 \\
185 \\
185\end{array}$ & $\begin{array}{l}509 \\
514 \\
520 \\
526\end{array}$ & $\begin{array}{l}178 \\
181 \\
183 \\
187\end{array}$ \\
\hline
\end{tabular}

See notes on first page of table. 


\begin{tabular}{|c|c|c|c|c|c|c|c|c|c|c|c|}
\hline \multirow{2}{*}{$\begin{array}{l}\text { Table } 1.5 \\
\text { England and Wales }\end{array}$} & \multicolumn{11}{|c|}{ Population: age, sex and legal marital status ${ }^{1}$} \\
\hline & \multirow{3}{*}{$\begin{array}{c}\text { Total } \\
\text { population }\end{array}$} & & & & & & & & & Numb & thousands) \\
\hline & & \multicolumn{5}{|c|}{ Males } & \multicolumn{5}{|c|}{ Females } \\
\hline Mid-year & & Single & Married & Divorced & Widowed & Total & Single & Married & Divorced & Widowed & Total \\
\hline \multicolumn{12}{|l|}{ Aged } \\
\hline \multicolumn{12}{|l|}{16 and over } \\
\hline 1971 & 36,818 & 4,173 & 12,522 & 187 & 682 & 17,563 & 3,583 & 12,566 & 296 & 2,810 & 19,255 \\
\hline 1976 & 37,486 & 4,369 & 12,511 & 376 & 686 & 17,941 & 3,597 & 12,538 & 533 & 2,877 & 19,545 \\
\hline 1981 & 38,724 & 5,013 & 12,238 & 611 & 698 & 18,559 & 4,114 & 12,284 & 828 & 2,939 & 20,165 \\
\hline 1986 & 39,837 & 5,625 & 11,867 & 917 & 695 & 19,103 & 4,617 & 12,000 & 1,165 & 2,953 & 20,734 \\
\hline 1991 & 40,501 & 5,891 & 11,636 & 1,187 & 727 & 19,441 & 4,817 & 11,833 & 1,459 & 2,951 & 21,060 \\
\hline 1996 & 40,827 & 6,225 & 11,310 & 1,346 & 733 & 19,614 & 5,168 & 11,433 & 1,730 & 2,881 & 21,212 \\
\hline 1999 & 41,325 & 6,582 & 11,143 & 1,433 & 732 & 19,890 & 5,526 & 11,235 & 1,875 & 2,800 & 21,435 \\
\hline 2000 & 41,569 & 6,721 & 11,113 & 1,456 & 731 & 20,022 & 5,650 & 11,199 & 1,927 & 2,772 & 21,547 \\
\hline 2001 & 41,865 & 6,894 & 11,090 & 1,482 & 733 & 20,198 & 5,798 & 11,150 & 1,975 & 2,745 & 21,667 \\
\hline 2002 & 42,135 & 7,062 & 11,043 & 1,524 & 730 & 20,358 & 5,944 & 11,094 & 2,031 & 2,709 & 21,777 \\
\hline 2003 & 42,409 & 7,226 & 10,995 & 1,571 & 726 & 20,517 & 6,102 & 11,033 & 2,087 & 2,669 & 21,892 \\
\hline 2004 & 42,731 & 7,419 & 10,941 & 1,617 & 722 & 20,700 & 6,279 & 10,980 & 2,144 & 2,628 & 22,031 \\
\hline 2005 & 43,141 & 7,623 & 10,923 & 1,662 & 719 & 20,927 & 6,486 & 10,943 & 2,198 & 2,588 & 22,214 \\
\hline 2006 & 43,494 & 7,833 & 10,881 & 1,696 & 716 & 21,126 & 6,683 & 10,893 & 2,244 & 2,548 & 22,367 \\
\hline 2007 & 43,860 & 8,049 & 10,851 & 1,724 & 715 & 21,338 & 6,871 & 10,851 & 2,289 & 2,511 & 22,521 \\
\hline \multicolumn{12}{|l|}{$16-19$} \\
\hline 1971 & 2,666 & 1,327 & 34 & 0 & 0 & 1,362 & 1,163 & 142 & 0 & 0 & 1,305 \\
\hline 1976 & 2,901 & 1,454 & 28 & 0 & 0 & 1,482 & 1,289 & 129 & 0 & 0 & 1,419 \\
\hline 1981 & 3,310 & 1,675 & 20 & 0 & 0 & 1,694 & 1,523 & 93 & 0 & 0 & 1,616 \\
\hline 1986 & 3,131 & 1,587 & 10 & 0 & 0 & 1,596 & 1,484 & 49 & 1 & 0 & 1,535 \\
\hline 1991 & 2,665 & 1,358 & 8 & 0 & 0 & 1,366 & 1,267 & 32 & 0 & 0 & 1,300 \\
\hline 1996 & 2,402 & 1,209 & 6 & 0 & 0 & 1,216 & 1,164 & 21 & 0 & 0 & 1,186 \\
\hline 1999 & 2,543 & 1,280 & 6 & 1 & 1 & 1,288 & 1,234 & 20 & 1 & 1 & 1,255 \\
\hline 2000 & 2,523 & 1,276 & 6 & 1 & 1 & 1,283 & 1,221 & 18 & 1 & 1 & 1,240 \\
\hline 2001 & 2,567 & 1,304 & 5 & 1 & 1 & 1,312 & 1,237 & 16 & 1 & 1 & 1,255 \\
\hline 2002 & 2,630 & 1,351 & 5 & 1 & 1 & 1,357 & 1,258 & 13 & 1 & 1 & 1,273 \\
\hline 2003 & 2,703 & 1,392 & 4 & 1 & 1 & 1,397 & 1,293 & 12 & 0 & 1 & 1,306 \\
\hline 2004 & 2,771 & 1,424 & 3 & 0 & 0 & 1,428 & 1,331 & 11 & 0 & 0 & 1,343 \\
\hline 2005 & 2,801 & 1,434 & 2 & 0 & 0 & 1,436 & 1,355 & 10 & 0 & 0 & 1,365 \\
\hline 2006 & 2,829 & 1,457 & 2 & 0 & 0 & $\begin{array}{l}1,459 \\
\text {. }\end{array}$ & 1,363 & 7 & 0 & 0 & 1,370 \\
\hline 2007 & 2,861 & 1,473 & 2 & 0 & 0 & 1,475 & 1,379 & 7 & 0 & 0 & 1,386 \\
\hline \multicolumn{12}{|l|}{$20-24$} \\
\hline 1971 & 3,773 & 1,211 & 689 & 3 & 0 & 1,904 & 745 & 1,113 & 9 & 2 & 1,869 \\
\hline 1976 & 3,395 & 1,167 & 557 & 4 & 0 & 1,728 & 725 & 925 & 16 & 2 & 1,667 \\
\hline 1981 & 3,744 & 1,420 & 466 & 10 & 1 & 1,896 & 1,007 & 811 & 27 & 2 & 1,847 \\
\hline 1986 & 4,171 & 1,768 & 317 & 14 & 0 & 2,099 & 1,383 & 657 & 32 & 1 & 2,072 \\
\hline 1991 & 3,911 & 1,717 & 242 & 12 & 0 & 1,971 & 1,421 & 490 & 29 & 1 & 1,941 \\
\hline 1996 & 3,291 & 1,538 & 117 & 3 & 0 & 1,658 & 1,361 & 260 & 11 & 1 & 1,633 \\
\hline 1999 & 3,047 & 1,449 & 78 & 2 & 0 & 1,530 & 1,320 & 188 & 8 & 1 & 1,517 \\
\hline 2000 & 3,088 & 1,470 & 74 & 3 & 0 & 1,548 & 1,352 & 180 & 8 & 1 & 1,540 \\
\hline 2001 & 3,157 & 1,501 & 74 & 3 & 1 & 1,579 & 1,390 & 178 & 8 & 1 & 1,578 \\
\hline 2002 & 3,212 & 1,530 & 73 & 3 & 1 & 1,606 & 1,427 & 170 & 8 & 1 & 1,606 \\
\hline 2003 & 3,281 & 1,568 & 74 & 3 & 1 & 1,645 & 1,459 & 166 & 8 & 1 & 1,636 \\
\hline 2004 & 3,376 & 1,632 & 75 & 3 & 1 & 1,712 & 1,491 & 163 & 8 & 2 & 1,664 \\
\hline 2005 & 3,477 & 1,693 & 73 & 3 & 1 & 1,771 & 1,539 & 157 & 8 & 2 & 1,706 \\
\hline 2006 & 3,558 & 1,741 & 67 & 3 & 1 & 1,812 & 1,591 & 146 & 7 & 1 & 1,746 \\
\hline 2007 & 3,661 & 1,813 & 64 & 3 & 1 & 1,881 & 1,637 & 136 & 6 & 1 & 1,780 \\
\hline $25-29$ & & & & & & & & & & & \\
\hline 1971 & 3,267 & 431 & 1,206 & 16 & 1 & 1,654 & 215 & 1,367 & 29 & 4 & 1,614 \\
\hline 1976 & 3,758 & 533 & 1,326 & 39 & 2 & 1,900 & 267 & 1,522 & 65 & 5 & 1,859 \\
\hline 1981 & 3,372 & 588 & 1,057 & 54 & 1 & 1,700 & 331 & 1,247 & 89 & 4 & 1,671 \\
\hline 1986 & 3,713 & 835 & 949 & 79 & 1 & 1,863 & 527 & 1,207 & 113 & 4 & 1,850 \\
\hline 1991 & 4,154 & 1,132 & 856 & 82 & 1 & 2,071 & 800 & 1,158 & 123 & 2 & 2,083 \\
\hline 1996 & 3,950 & 1,273 & 650 & 46 & 1 & 1,970 & 977 & 906 & 93 & 3 & 1,980 \\
\hline 1999 & 3,687 & 1,304 & 497 & 34 & 1 & 1,836 & 1,051 & 725 & 72 & 3 & 1,851 \\
\hline 2000 & 3,605 & 1,305 & 459 & 31 & 1 & 1,796 & 1,065 & 677 & 65 & 3 & 1,810 \\
\hline 2001 & 3,487 & 1,293 & 420 & 28 & 1 & 1,742 & 1,059 & 625 & 58 & 3 & 1,745 \\
\hline 2002 & 3,365 & 1,277 & 384 & 26 & 1 & 1,688 & 1,049 & 574 & 52 & 3 & 1,676 \\
\hline 2003 & 3,284 & 1,265 & 356 & 24 & 1 & 1,647 & 1,051 & 536 & 48 & 2 & 1,638 \\
\hline 2004 & 3,280 & 1,278 & 339 & 23 & 1 & 1,641 & 1,078 & 513 & 46 & 2 & 1,639 \\
\hline 2005 & 3,354 & 1,320 & 331 & 23 & 1 & 1,675 & 1,128 & 504 & 45 & 2 & 1,679 \\
\hline 2006 & 3,434 & 1,371 & 324 & 22 & 1 & 1,718 & 1,178 & 490 & 45 & 2 & 1,716 \\
\hline 2007 & 3,526 & 1,422 & 326 & 22 & 1 & 1,770 & 1,223 & 486 & 44 & 2 & 1,756 \\
\hline
\end{tabular}

1 Rates have been revised from 2002 to include the adjustments for marriages to England and Wales residents occurring abroad - see 'In Brief'. 


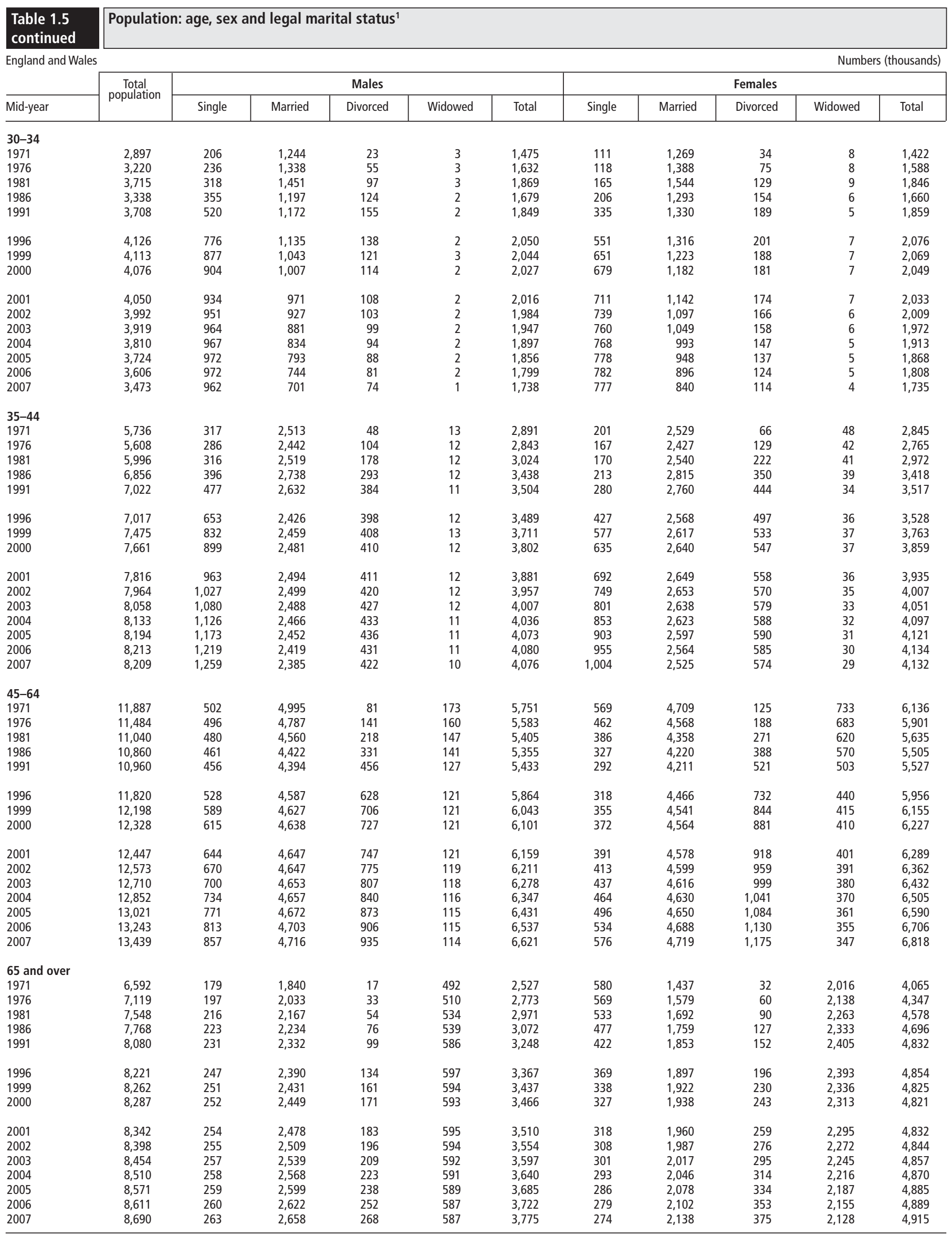


Constituent countries of the United Kingdom

\begin{tabular}{|c|c|c|c|c|c|c|c|c|c|c|c|}
\hline \multicolumn{11}{|c|}{ Constituent countries of the United Kingdom } & Numbers (thousands) \\
\hline \multirow{3}{*}{$\begin{array}{l}\text { Mid-year to } \\
\text { mid-year }\end{array}$} & \multirow{3}{*}{$\begin{array}{l}\text { Population at } \\
\text { start of period }\end{array}$} & \multirow{3}{*}{$\begin{array}{l}\text { Total } \\
\text { annual } \\
\text { change }\end{array}$} & \multicolumn{8}{|c|}{ Components of change (mid-year to mid-year or annual averages) } & \multirow{3}{*}{$\begin{array}{c}\text { Population at } \\
\text { end of } \\
\text { period }\end{array}$} \\
\hline & & & \multirow{2}{*}{$\begin{array}{l}\text { Live } \\
\text { births }\end{array}$} & \multirow[t]{2}{*}{ Deaths } & \multirow{2}{*}{$\begin{array}{c}\text { Natural } \\
\text { change } \\
\text { (Live births - } \\
\text { deaths) }\end{array}$} & \multicolumn{4}{|c|}{ Net civilian migration } & \multirow{2}{*}{$\begin{array}{l}\text { Other } \\
\text { changes }\end{array}$} & \\
\hline & & & & & & Total $^{1}$ & $\begin{array}{c}\text { To/from } \\
\text { rest of UK }\end{array}$ & $\begin{array}{c}\text { To/from } \\
\text { Irish Republic }\end{array}$ & $\begin{array}{l}\text { To/from } \\
\text { rest of the } \\
\text { world }\end{array}$ & & \\
\hline
\end{tabular}

\section{United Kingdom}

$1971-76$

$1976-81$
$1981-86$

1981-86

1991-96

1996-01

2001-02

2002-03

2003-04

2004-05

2005-06

England and Wales

971-76

1976-81

981-86

$1986-91$
$1991-96$

1996-01

2001-02

2002-03

2003-04

2005-06

2006-07

England

1971-76

1976-81

1986-91

1991-96

1996-01

2001-02

2002-03

2004-05

2005-06

Wales

1971-76

1976-81

1981-86

1986-91

1996-01

2001-02

2003-04

2004-05

2005-06

2006-07

Scotland

1971-76
1976-81

1981-86

1986-91

1991-96

1996-01

2001-02

2002-03

2003-04

2004-05

2005-06

Northern Ireland

1971-76

1976-81

$1981-86$

1991-96

55,928

56,216
56,357

56,684

57,439

+58
$+\quad 27$

$+\quad 27$
+65
+

$+148$

58,164

59,113
59,323

59,323
59,557

59,846

60,238

60,587

$+190$

$+210$

$+289$

$+349$

$\begin{array}{ll}49,152 & +61 \\ 49,459 & +35 \\ 49,634 & +73\end{array}$

$\begin{array}{ll}49,999 & +150 \\ 50,748 & +132\end{array}$

51,410

52,360

52,572
52,797

53,057

53,419
53,729

766
705
733
782
756

$670+96$
$662+$

$\begin{array}{lll}670 & +96 & -55 \\ 662 & +42 & -33 \\ 662 & +70 & -5\end{array}$

$647++135$

$\begin{aligned}+135 & +13 \\ +117 & +29\end{aligned}$

-
-
-
-

56,216

5,684

57,439

$639+11$

$\begin{array}{ll}706 & 623 \\ 663 & 601\end{array}$

$+83+107$

+62
+77

+104
+127

+127
+159
+187

$+157$

605
603
591

591
575
571

$+185$

$\begin{array}{ll}+187 & +190 \\ & +201\end{array}$

76
$+\quad 30$

+
+30
+57

+57
+120
+106

-28
$-\quad 9$
+16
+30
+27
+112
+151
+149
+159
+241
+159
+166

$$
\begin{array}{r}
+10 \\
+11
\end{array}
$$

\begin{tabular}{rrrr}
\hline-9 & -29 & +13 & 49,459 \\
-3 & -17 & +14 & 49,634 \\
.. &.. &.. & 49,999 \\
.. &.. &.. & 50,748 \\
.. &.. &.. & 51,410 \\
. &. &. & 52,360 \\
. &. &.. & 52,572 \\
.. &.. &.. & 52,797 \\
.. &.. &.. & 53,057 \\
.. &.. &.. & 53,419 \\
.. &.. &.. & 53,729 \\
.. &.. &.. & 54,072 \\
.. &. &. &
\end{tabular}

46,412

46,660

46,82

47,188
47,875

+50
+32
+73
+137

$\begin{array}{ll}48,519 & +186 \\ 49,450 & +203\end{array}$

652

49,866

49,866
50,111
50,466

50,466
50,763

+214
+214
+245

+245
+355
+

+357
+297
+329

$2,740+12$

2,790
2,813

2,811

+12
$+\quad 3$
$-\quad 1$

-
+12
$+\quad 4$

$2,891+4$

$\begin{array}{ll}2,910 & +10 \\ 2,920 & +11\end{array}$

$\begin{array}{ll}2,920 & +11 \\ 2,931 & +15\end{array}$

2,946

$2,954+12$

5,236

$\begin{array}{ll}5,233 & -11 \\ 5,180 & -14\end{array}$

$\begin{array}{ll}5,112 & - \\ 5,083 & +\end{array}$

$\begin{array}{ll}5,092 & -6 \\ 5,064 & -9\end{array}$

$\begin{array}{rr}5,064 & -9 \\ 5,055 & +3 \\ 5,057 & +21\end{array}$

$\begin{array}{ll}5,057 & +21 \\ 5,078 & +16\end{array}$

$\begin{array}{ll}5,078 & +16 \\ 5,095 & +22 \\ 5,117 & +27\end{array}$

$\begin{array}{ll}627 & 552 \\ 577 & 546 \\ 603 & 547 \\ 651 & 535 \\ 632 & 528 \\ 593 & 514 \\ 560 & 497 \\ 578 & 498 \\ 600 & 498 \\ 608 & 487 \\ 623 & 474 \\ 643 & 469\end{array}$

552
546
547
535
528
514
497
498
498
487
474
469

+75
+31
+56
+116

-35
-11
+18
+21
+24
+107
+139
+135
+143
+234
+148
+155

+1
+6
..
..
..
..
..
..
..
..
..
..

-9
-3
..
..
..
.
..
..
..
.
.
.
..

$-27$

+10
+12
.+

46,660

46,821

47,188
47,875

48,519

$\begin{array}{ll}+79 & +107 \\ +63 & +139 \\ +79 & +135 \\ +102 & +143 \\ +121 & +234 \\ +149 & +148 \\ +174 & +155\end{array}$

$\begin{array}{ll}37 & 36 \\ 35 & 36 \\ 36 & 35 \\ 38 & 34 \\ 36 & 35\end{array}$

$\begin{array}{rrrr}+1 & +7 & +10 \\ -1 & +2 & +5 \\ +1 & -1 & . . \\ +4 & +8 & . .\end{array}$

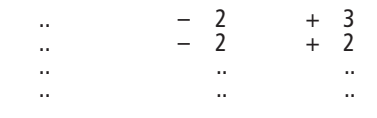

2,799

2,813

2,873

2,910

$\begin{array}{ll}-1 & +5 \\ - & +\end{array}$

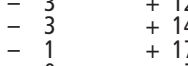

0
$+\quad 2$
$+\quad 2$

$+\quad 7$
$+\quad 10$
+

0
11
14
6
2
6
9
3
21
16
22
27

73
66
66
66

$\begin{array}{ll}64 & + \\ 64 & + \\ 64 & + \\ 62 & + \\ 61 & +\end{array}$

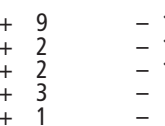

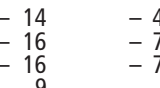

-4
-7
-7

-10
$-\quad 10$
$-\quad 7$

9,450
9,652

49,866

50,466

0,763

,092

2,931

2,946

2,966

5233

5,180

5,112
5,083

5,092

5,064

$\begin{array}{ll}-3 & -3 \\ -6 & -3\end{array}$

$-7+9$
-4

$\begin{array}{rr}-4 & +25 \\ -\quad 2 & +19\end{array}$

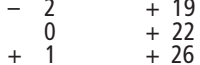

5,057

5,095

5,117

$\begin{array}{lll}1,540 & -3 & 28 \\ 1,524 & +3 & 27 \\ 1,543 & +6 & 28 \\ 1,574 & +7 & 27 \\ 1,607 & +11 & 25\end{array}$

28
27
28
27
25

$\begin{array}{ll}17 & + \\ 17 & + \\ 16 & + \\ 16 & + \\ 15 & +\end{array}$

$\begin{array}{ll}+11 & - \\ +10 & - \\ +12 & - \\ +12 & - \\ +9 & +\end{array}$

$\begin{array}{ll}-14 & -7 \\ -\quad 8 & -4 \\ -\quad 5 & -3 \\ -\quad 5 & -3 \\ +\quad 2 & \end{array}$

$-\quad 7$
$-\quad 3$
$-\quad 1$
$-\quad 1$

1
$+\quad 17$
-7

1,524
1,543
1,574
1,607
1,662
1,689
1,697
1,703
1,710
1,724
1,742
1,759

1 For UK, England, Wales and Scotland from 1981 onwards, this column is not an estimate of net civilian migration; it also includes "other" changes. It has been derived by subtraction using revised population estimates and natural change. 


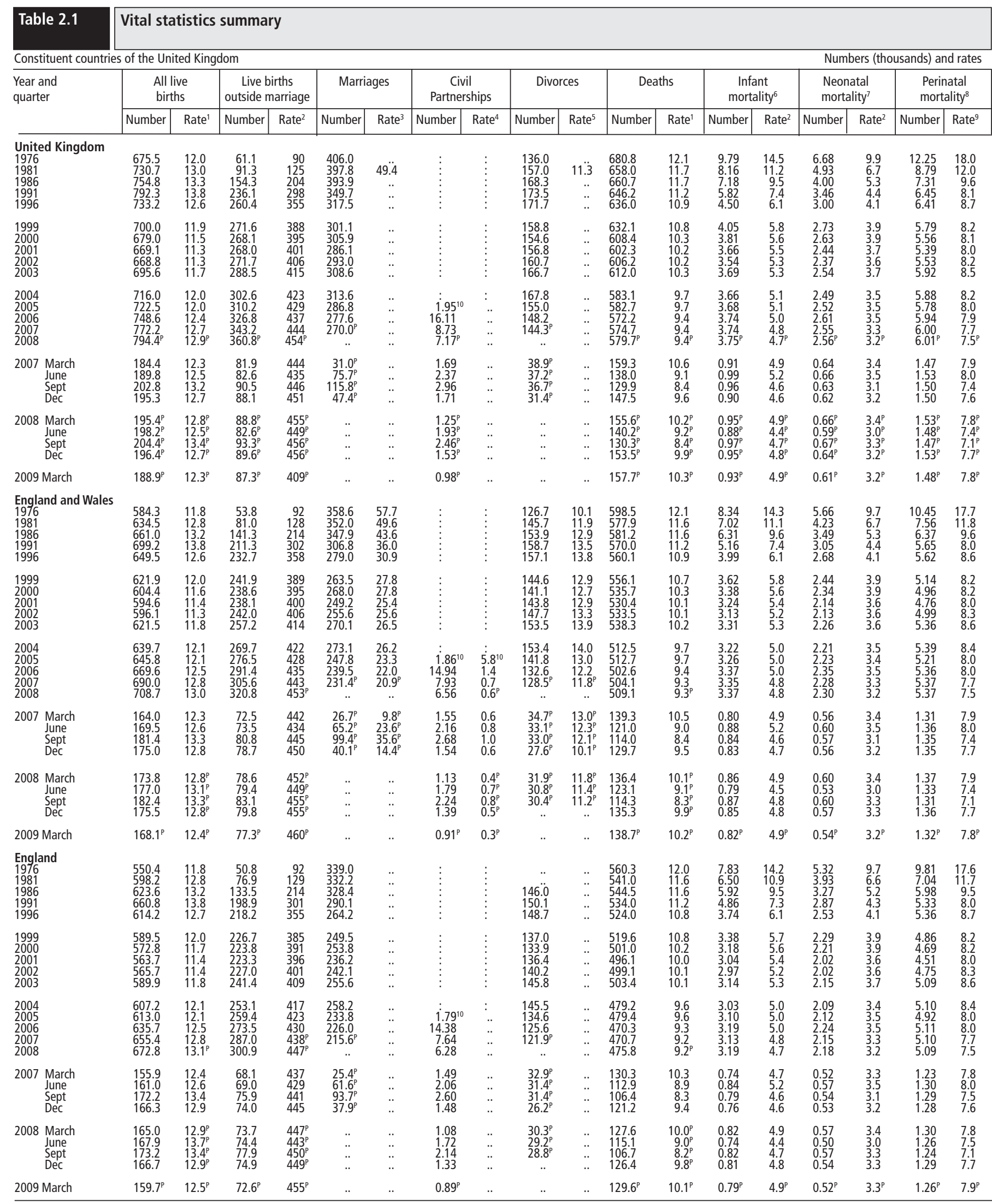

Note: Death figures for England and Wales represent the number of deaths registered in each year up to 1992 , and the number of deaths occurring in each year from 1993 to 2005. Death figures for 2006, 2007 and provisional death figures for 2008 relate to registrations.

Birth and death figures for England and also for Wales each exclude events for persons usually resident outside England and Wales. These events are, however, included in the totals for England and Wales combined, and for the United Kingdom.

2007 Perinatal figures have been updated to include late receipt of stillbirth records.

From 1981 births to non-resident mothers in Northern Ireland are excluded from the figures for Northern Ireland, and for the United Kingdom.
From August 2009, Scotland revised their divorce figures back to 1985. These are based on the annual 'Date of decree' and exclude late divorces from previous years. Infant, neonatal and perinatal mortality rates for Northern Ireland have now been amended to take account of the non-resident livebirths.

The birth and death rates for 2008 and 2009 are based on 2006-based population The birth and death rates for 2008 and 2009 are based on 2006-based population
projections for 2008 and 2009. Marriage, civil partnership and divorce rates for 2008 projections for 2008 and 2009. Marriage, civil partnership and divorce rates for 2008 2002 to include the adjustments for marriages to England and Wales residents occurring abroad. Marital status estimates/rates are not available for Northern Ireland/UK. 


\begin{tabular}{|c|c|c|c|c|c|c|c|c|c|c|c|c|c|c|c|c|c|c|c|}
\hline \multicolumn{2}{|c|}{$\begin{array}{l}\text { Table } 2.1 \\
\text { continued }\end{array}$} & \multicolumn{18}{|c|}{ Vital statistics summary } \\
\hline \multicolumn{16}{|c|}{ Constituent countries of the United Kingdom } & \multicolumn{4}{|c|}{ Numbers (thousands) and rates } \\
\hline \multirow{2}{*}{\multicolumn{2}{|c|}{$\begin{array}{l}\text { Year and } \\
\text { quarter }\end{array}$}} & \multicolumn{2}{|c|}{$\begin{array}{l}\text { All live } \\
\text { births }\end{array}$} & \multicolumn{2}{|c|}{$\begin{array}{c}\text { Live births } \\
\text { outside marriage }\end{array}$} & \multicolumn{2}{|c|}{ Marriages } & \multicolumn{2}{|c|}{$\begin{array}{c}\text { Civil } \\
\text { Partnerships }\end{array}$} & \multicolumn{2}{|c|}{ Divorces } & \multicolumn{2}{|c|}{ Deaths } & \multicolumn{2}{|c|}{$\begin{array}{c}\text { Infant } \\
\text { mortality }\end{array}$} & \multicolumn{2}{|c|}{$\begin{array}{l}\text { Neonatal } \\
\text { mortality }\end{array}$} & \multicolumn{2}{|c|}{$\begin{array}{l}\text { Perinatal } \\
\text { mortality }\end{array}$} \\
\hline & & Number & Rate $^{1}$ & Number & Rate $^{2}$ & Number & Rate $^{3}$ & Number & Rate $^{4}$ & Number & Rate $^{5}$ & Number & Rate $^{1}$ & Number & Rate $^{2}$ & Number & Rate $^{2}$ & Number & Rate $^{9}$ \\
\hline $\begin{array}{l}\text { Wale } \\
1976 \\
1981 \\
1986 \\
1991 \\
1996\end{array}$ & & $\begin{array}{l}33.4 \\
35.8 \\
33.0 \\
38.1 \\
34.9\end{array}$ & $\begin{array}{l}11.9 \\
12.7 \\
13.2 \\
13.3 \\
12.1\end{array}$ & $\begin{array}{r}2.9 \\
4.0 \\
7.8 \\
12.3 \\
14.4\end{array}$ & $\begin{array}{r}86 \\
112 \\
211 \\
323 \\
412\end{array}$ & $\begin{array}{l}19.5 \\
19.8 \\
19.5 \\
16.6 \\
14.8\end{array}$ & $\begin{array}{l}. . \\
\ddot{.} \\
. .\end{array}$ & $\vdots$ & $\begin{array}{l}\vdots \\
\vdots \\
\vdots\end{array}$ & $\begin{array}{c}. . \\
7.8 \\
8.4 \\
8.4\end{array}$ & $\begin{array}{l}. . \\
\ddot{.} \\
. .\end{array}$ & $\begin{array}{l}36.3 \\
35.0 \\
34.7 \\
34.1 \\
34.6\end{array}$ & $\begin{array}{l}13.0 \\
12.4 \\
12.3 \\
11.9 \\
12.0\end{array}$ & $\begin{array}{l}0.46 \\
0.45 \\
0.35 \\
0.25 \\
0.20\end{array}$ & $\begin{array}{r}13.7 \\
12.6 \\
9.5 \\
6.6 \\
5.6\end{array}$ & $\begin{array}{l}0.32 \\
0.29 \\
0.21 \\
0.16 \\
0.13\end{array}$ & $\begin{array}{l}9.6 \\
8.1 \\
5.6 \\
4.1 \\
3.6\end{array}$ & $\begin{array}{l}0.64 \\
0.51 \\
0.38 \\
0.30 \\
0.26\end{array}$ & $\begin{array}{r}19.0 \\
14.1 \\
10.3 \\
7.9 \\
7.5\end{array}$ \\
\hline $\begin{array}{l}1999 \\
2000 \\
2001 \\
2002 \\
2003\end{array}$ & & $\begin{array}{l}32.1 \\
31.3 \\
30.6 \\
30.2 \\
31.4\end{array}$ & $\begin{array}{l}11.1 \\
10.8 \\
10.5 \\
10.3 \\
10.7\end{array}$ & $\begin{array}{l}14.8 \\
14.8 \\
14.8 \\
15.0 \\
15.8\end{array}$ & $\begin{array}{l}461 \\
472 \\
483 \\
497 \\
503\end{array}$ & $\begin{array}{l}14.0 \\
14.1 \\
13.0 \\
13.5 \\
14.5\end{array}$ & $\begin{array}{l}. . \\
. . \\
. . \\
. .\end{array}$ & $\vdots$ & $\vdots$ & $\begin{array}{l}7.5 \\
7.2 \\
7.4 \\
7.6 \\
7.7\end{array}$ & $\begin{array}{l}. . \\
. . \\
. . \\
. .\end{array}$ & $\begin{array}{l}35.0 \\
33.3 \\
33.0 \\
33.2 \\
33.7\end{array}$ & $\begin{array}{l}12.1 \\
11.5 \\
11.3 \\
11.4 \\
11.5\end{array}$ & $\begin{array}{l}0.20 \\
0.17 \\
0.16 \\
0.14 \\
0.13\end{array}$ & $\begin{array}{l}6.1 \\
5.3 \\
5.4 \\
4.5 \\
4.3\end{array}$ & $\begin{array}{l}0.13 \\
0.11 \\
0.11 \\
0.10 \\
0.10\end{array}$ & $\begin{array}{l}4.0 \\
3.5 \\
3.5 \\
3.2 \\
3.1\end{array}$ & $\begin{array}{l}0.25 \\
0.23 \\
0.23 \\
0.24 \\
0.24\end{array}$ & $\begin{array}{l}7.7 \\
7.2 \\
7.5 \\
7.7 \\
7.6\end{array}$ \\
\hline $\begin{array}{l}2004 \\
2005 \\
2006 \\
2007 \\
2008\end{array}$ & & $\begin{array}{l}32.3 \\
32.6 \\
33.6 \\
34.4 \\
35.7\end{array}$ & $\begin{array}{l}11.0 \\
11.0 \\
11.3 \\
11.5 \\
11.9^{p}\end{array}$ & $\begin{array}{l}16.6 \\
17.1 \\
17.8 \\
18.5 \\
19.8\end{array}$ & $\begin{array}{l}513 \\
524 \\
530 \\
538 \\
556^{\mathrm{p}}\end{array}$ & $\begin{array}{c}14.9 \\
14.0 \\
13.5 \\
12.8^{p} \\
. .\end{array}$ & $\begin{array}{l}. . \\
. . \\
. . \\
. .\end{array}$ & $\begin{array}{l}0.077^{10} \\
0.56 \\
0.29 \\
0.28\end{array}$ & $\begin{array}{l}: \\
. . \\
. . \\
. .\end{array}$ & $\begin{array}{l}7.9 \\
7.2 \\
7.0^{p} \\
6.7^{p} \\
. .\end{array}$ & $\begin{array}{l}. . \\
. . \\
. . \\
. .\end{array}$ & $\begin{array}{l}32.1 \\
32.1 \\
31.1 \\
32.1 \\
32.1\end{array}$ & $\begin{array}{l}10.9 \\
10.9 \\
10.5 \\
10.8 \\
10.7^{\mathrm{p}}\end{array}$ & $\begin{array}{l}0.16 \\
0.13 \\
0.14 \\
0.18 \\
0.15\end{array}$ & $\begin{array}{l}4.9 \\
4.1 \\
4.1 \\
5.3 \\
4.1\end{array}$ & $\begin{array}{l}0.10 \\
0.09 \\
0.09 \\
0.12 \\
0.11\end{array}$ & $\begin{array}{l}3.1 \\
2.9 \\
2.8 \\
3.4 \\
3.0\end{array}$ & $\begin{array}{l}0.26 \\
0.24 \\
0.23 \\
0.25 \\
0.24\end{array}$ & $\begin{array}{l}8.0 \\
7.4 \\
6.9 \\
7.3 \\
6.7\end{array}$ \\
\hline 2007 & $\begin{array}{l}\text { March } \\
\text { June } \\
\text { Sept } \\
\text { Dec }\end{array}$ & $\begin{array}{l}8.1 \\
8.5 \\
9.1 \\
8.7\end{array}$ & $\begin{array}{l}11.0 \\
11.4 \\
12.1 \\
11.6\end{array}$ & $\begin{array}{l}4.3 \\
4.5 \\
4.9 \\
4.8\end{array}$ & $\begin{array}{l}536 \\
530 \\
541 \\
547\end{array}$ & $\begin{array}{l}1.3^{p} \\
3.6^{p} \\
5.7^{p} \\
2.2^{p}\end{array}$ & $\begin{array}{l}. . \\
. . \\
. .\end{array}$ & $\begin{array}{l}0.06 \\
0.10 \\
0.08 \\
0.06\end{array}$ & $\begin{array}{l}. . \\
. . \\
. .\end{array}$ & $\begin{array}{l}1.8^{p} \\
1.8^{p} \\
1.7^{p}\end{array}$ & $\begin{array}{l}. . \\
. . \\
. .\end{array}$ & $\begin{array}{l}8.8 \\
7.9 \\
7.3 \\
8.3\end{array}$ & $\begin{array}{r}11.9 \\
10.6 \\
91.7 \\
11.0\end{array}$ & $\begin{array}{l}0.05 \\
0.04 \\
0.04 \\
0.05\end{array}$ & $\begin{array}{l}6.3 \\
4.5 \\
4.4 \\
6.1\end{array}$ & $\begin{array}{l}0.03 \\
0.02 \\
0.03 \\
0.04\end{array}$ & $\begin{array}{l}3.7 \\
2.8 \\
2.8 \\
4.1\end{array}$ & $\begin{array}{l}0.07 \\
0.06 \\
0.05 \\
0.07\end{array}$ & $\begin{array}{l}9.0 \\
6.8 \\
57.7 \\
7.9\end{array}$ \\
\hline 2008 & $\begin{array}{l}3 \text { March } \\
\text { June } \\
\text { Sept } \\
\text { Dec }\end{array}$ & $\begin{array}{l}8.7 \\
9.0 \\
9.2 \\
8.8\end{array}$ & $\begin{array}{l}11.8^{p} \\
12.0^{p} \\
12.2^{p} \\
11.6^{p}\end{array}$ & $\begin{array}{l}4.8 \\
5.0 \\
5.1 \\
4.9\end{array}$ & $\begin{array}{l}555^{p} \\
55^{p} \\
55^{p} \\
561^{p}\end{array}$ & $\begin{array}{l}. . \\
. . \\
. .\end{array}$ & $\begin{array}{l}. . \\
. . \\
. .\end{array}$ & $\begin{array}{l}0.06 \\
0.07 \\
0.10 \\
0.06\end{array}$ & $\begin{array}{l}. . \\
. . \\
.\end{array}$ & $\begin{array}{l}1.6^{p} \\
1.5^{p} \\
1.6^{p} \\
. .\end{array}$ & $\begin{array}{l}. . \\
. . \\
. .\end{array}$ & $\begin{array}{l}8.6 \\
7.7 \\
7.3 \\
8.5\end{array}$ & $\begin{array}{r}11.5^{p} \\
10.4^{p} \\
916^{p} \\
11.3^{p}\end{array}$ & $\begin{array}{l}0.04 \\
0.04 \\
0.04 \\
0.04\end{array}$ & $\begin{array}{l}4.1 \\
4.2 \\
3.8 \\
4.3\end{array}$ & $\begin{array}{l}0.03 \\
0.03 \\
0.03 \\
0.03\end{array}$ & $\begin{array}{l}3.0 \\
3.2 \\
2.9 \\
3.0\end{array}$ & $\begin{array}{l}0.06 \\
0.06 \\
0.06 \\
0.06\end{array}$ & $\begin{array}{l}7.3 \\
6.4 \\
6.3 \\
6.9\end{array}$ \\
\hline 2009 & March & $8.4^{\mathrm{p}}$ & $11.3^{p}$ & $4.7^{\mathrm{P}}$ & $559^{p}$ & .. & .. & $0.03^{p}$ & .. & .. & .. & $8.9^{\mathrm{P}}$ & $12.0^{p}$ & $0.03^{p}$ & $3.1^{p}$ & $0.02^{p}$ & $2.0^{p}$ & $0.05^{p}$ & $5.3^{p}$ \\
\hline $\begin{array}{l}\text { Scotl } \\
1976 \\
1981 \\
1986 \\
1991 \\
1996\end{array}$ & & $\begin{array}{l}64.9 \\
69.1 \\
65.8 \\
67.0 \\
59.3\end{array}$ & $\begin{array}{l}12.5 \\
13.4 \\
12.9 \\
13.2 \\
11.6\end{array}$ & $\begin{array}{r}6.0 \\
8.5 \\
13.6 \\
19.5 \\
21.4\end{array}$ & $\begin{array}{r}93 \\
122 \\
206 \\
291 \\
360\end{array}$ & $\begin{array}{l}37.5 \\
36.2 \\
35.8 \\
33.8 \\
30.2\end{array}$ & $\begin{array}{l}53.8 \\
47.5 \\
42.9 \\
39.0 \\
33.2\end{array}$ & $\vdots$ & $\begin{array}{l}\vdots \\
\vdots \\
\vdots\end{array}$ & $\begin{array}{r}8.1 \\
9.9 \\
12.8 \\
12.4 \\
12.3\end{array}$ & $\begin{array}{r}6.5 \\
8.0 \\
10.7 \\
10.6 \\
10.9\end{array}$ & $\begin{array}{l}65.3 \\
63.8 \\
63.5 \\
61.0 \\
60.7\end{array}$ & $\begin{array}{l}12.5 \\
12.3 \\
12.4 \\
12.0 \\
11.9\end{array}$ & $\begin{array}{l}0.96 \\
0.78 \\
0.58 \\
0.47 \\
0.37\end{array}$ & $\begin{array}{r}14.8 \\
11.3 \\
8.8 \\
7.1 \\
6.2\end{array}$ & $\begin{array}{l}0.67 \\
0.47 \\
0.34 \\
0.29 \\
0.23\end{array}$ & $\begin{array}{r}10.3 \\
6.9 \\
5.2 \\
4.6 \\
3.9\end{array}$ & $\begin{array}{l}1.20 \\
0.81 \\
0.67 \\
0.58 \\
0.55\end{array}$ & $\begin{array}{r}18.3 \\
11.6 \\
10.2 \\
8.6 \\
9.2\end{array}$ \\
\hline $\begin{array}{l}1999 \\
2000 \\
2001 \\
2002 \\
2003\end{array}$ & & $\begin{array}{l}55.1 \\
53.1 \\
52.5 \\
55.3 \\
52.4\end{array}$ & $\begin{array}{l}10.9 \\
10.5 \\
10.4 \\
10.1 \\
10.4\end{array}$ & $\begin{array}{l}22.7 \\
22.6 \\
22.8 \\
22.5 \\
23.9\end{array}$ & $\begin{array}{l}412 \\
426 \\
433 \\
440 \\
455\end{array}$ & $\begin{array}{l}29.9 \\
30.4 \\
29.6 \\
29.8 \\
30.8\end{array}$ & $\begin{array}{l}31.5 \\
31.6 \\
31.0 \\
30.8 \\
31.3\end{array}$ & $\vdots$ & $\begin{array}{c}\vdots \\
\vdots \\
\vdots\end{array}$ & $\begin{array}{l}11.9 \\
11.1 \\
10.7 \\
10.9 \\
10.9\end{array}$ & $\begin{array}{r}10.9 \\
10.3 \\
9.7 \\
10.0 \\
10.2\end{array}$ & $\begin{array}{l}60.3 \\
57.8 \\
57.4 \\
58.1 \\
58.5\end{array}$ & $\begin{array}{l}11.9 \\
11.4 \\
11.3 \\
11.5 \\
11.6\end{array}$ & $\begin{array}{l}0.28 \\
0.31 \\
0.29 \\
0.27 \\
0.27\end{array}$ & $\begin{array}{l}5.0 \\
5.7 \\
5.5 \\
5.3 \\
5.1\end{array}$ & $\begin{array}{l}0.18 \\
0.21 \\
0.20 \\
0.16 \\
0.18\end{array}$ & $\begin{array}{l}3.3 \\
4.0 \\
3.8 \\
3.2 \\
3.4\end{array}$ & $\begin{array}{l}0.42 \\
0.45 \\
0.45 \\
0.39 \\
0.42\end{array}$ & $\begin{array}{l}7.6 \\
8.4 \\
8.5 \\
7.6 \\
8.0\end{array}$ \\
\hline $\begin{array}{l}2004 \\
2005 \\
2006 \\
2007 \\
2008\end{array}$ & & $\begin{array}{l}54.0 \\
54.4 \\
55.7 \\
57.8 \\
60.0\end{array}$ & $\begin{array}{l}10.6 \\
10.7 \\
10.9 \\
11.2 \\
11.6^{p}\end{array}$ & $\begin{array}{l}25.2 \\
25.6 \\
26.6 \\
28.4 \\
30.1\end{array}$ & $\begin{array}{l}467 \\
471 \\
477 \\
491 \\
501^{p}\end{array}$ & $\begin{array}{r}32.2 \\
30.9 \\
29.9 \\
29.9 \\
28.9\end{array}$ & $\begin{array}{l}32.1 \\
30.3 \\
28.7 \\
28.1 \\
27.2^{p}\end{array}$ & $\begin{array}{l}0.08^{10} \\
1.05 \\
0.69 \\
0.53\end{array}$ & $\begin{array}{l}2.5^{10} \\
1.0 \\
0.6 \\
0.5^{p}\end{array}$ & $\begin{array}{l}11.3 \\
10.9 \\
13.1 \\
12.8 \\
11.5\end{array}$ & $\begin{array}{l}10.5 \\
10.3 \\
12.3 \\
12.2 \\
10.9^{p}\end{array}$ & $\begin{array}{l}56.2 \\
55.7 \\
55.1 \\
56.0 \\
55.7\end{array}$ & $\begin{array}{l}11.1 \\
11.0 \\
10.8 \\
10 . .^{p} \\
10.8^{p}\end{array}$ & $\begin{array}{l}0.27 \\
0.28 \\
0.25 \\
0.27 \\
0.25\end{array}$ & $\begin{array}{l}4.9 \\
5.2 \\
4.5 \\
4.7 \\
4.2\end{array}$ & $\begin{array}{l}0.17 \\
0.19 \\
0.17 \\
0.19 \\
0.17\end{array}$ & $\begin{array}{l}3.1 \\
3.5 \\
3.1 \\
3.2 \\
2.8\end{array}$ & $\begin{array}{l}0.44 \\
0.42 \\
0.42 \\
0.45 \\
0.45\end{array}$ & $\begin{array}{l}8.1 \\
7.7 \\
7.4 \\
7.8 \\
7.4\end{array}$ \\
\hline 2007 & $\begin{array}{l}\text { March } \\
\text { June } \\
\text { Sept } \\
\text { Dec }\end{array}$ & $\begin{array}{l}14.2 \\
14.3 \\
14.9 \\
14.4\end{array}$ & $\begin{array}{l}11.2 \\
11.1 \\
11.5 \\
11.1\end{array}$ & $\begin{array}{l}7.1 \\
6.9 \\
7.2 \\
7.1\end{array}$ & $\begin{array}{l}501 \\
482 \\
485 \\
497\end{array}$ & $\begin{array}{r}3.3 \\
8.1 \\
12.6 \\
5.8\end{array}$ & $\begin{array}{l}12.7 \\
30.7 \\
47.1 \\
21.6\end{array}$ & $\begin{array}{l}0.11 \\
0.18 \\
0.25 \\
0.15\end{array}$ & $\begin{array}{l}0.4 \\
0.7 \\
0.9 \\
0.6\end{array}$ & $\begin{array}{l}3.3 \\
3.3 \\
3.0 \\
3.1\end{array}$ & $\begin{array}{l}12.8 \\
12.7 \\
11.4 \\
11.8\end{array}$ & $\begin{array}{l}15.8 \\
13.4 \\
12.6 \\
1.2\end{array}$ & $\begin{array}{r}12.5 \\
10.4 \\
9.7 \\
10.9\end{array}$ & $\begin{array}{l}0.07 \\
0.08 \\
0.07 \\
0.06\end{array}$ & $\begin{array}{l}4.9 \\
5.3 \\
4.6 \\
3.9\end{array}$ & $\begin{array}{l}0.05 \\
0.05 \\
0.05 \\
0.04\end{array}$ & $\begin{array}{l}3.6 \\
3.4 \\
3.0 \\
2.9\end{array}$ & $\begin{array}{l}0.12 \\
0.12 \\
0.11 \\
0.11\end{array}$ & $\begin{array}{l}8.1 \\
8.6 \\
7.1 \\
7.5\end{array}$ \\
\hline 2008 & $\begin{array}{l}3 \text { March } \\
\text { June } \\
\text { Sept } \\
\text { Dec }\end{array}$ & $\begin{array}{l}15.1 \\
14.9 \\
15.5 \\
14.5\end{array}$ & $\begin{array}{l}11.8^{p} \\
11.6^{p} \\
12.1^{p} \\
11.2^{p}\end{array}$ & $\begin{array}{l}7.7 \\
7.3 \\
7.7 \\
7.4\end{array}$ & $\begin{array}{l}510^{p} \\
488^{p} \\
498^{p} \\
507^{p}\end{array}$ & $\begin{array}{r}3.4 \\
7.9 \\
11.9 \\
5.7\end{array}$ & $\begin{array}{l}13.0^{p} \\
29.9^{p} \\
44 . .^{p} \\
21.2^{p}\end{array}$ & $\begin{array}{l}0.10 \\
0.11 \\
0.20 \\
0.12\end{array}$ & $\begin{array}{l}0.4^{p} \\
0.4^{p} \\
0.7^{p} \\
0.4^{p}\end{array}$ & $\begin{array}{l}2.8 \\
3.0 \\
2.8 \\
2.8\end{array}$ & $\begin{array}{l}10.6^{p} \\
11.6^{p} \\
10.8^{p} \\
10.7^{p}\end{array}$ & $\begin{array}{l}15.1 \\
13.5 \\
12.6 \\
14.5\end{array}$ & $\begin{array}{r}11.8^{p} \\
10.5^{p} \\
917^{p} \\
11.2^{p}\end{array}$ & $\begin{array}{l}0.06 \\
0.06 \\
0.07 \\
0.06\end{array}$ & $\begin{array}{l}3.9 \\
4.0 \\
4.8 \\
4.1\end{array}$ & $\begin{array}{l}0.04 \\
0.04 \\
0.05 \\
0.04\end{array}$ & $\begin{array}{l}2.8 \\
2.5 \\
3.4 \\
2.4\end{array}$ & $\begin{array}{l}0.11 \\
0.11 \\
0.11 \\
0.12\end{array}$ & $\begin{array}{l}7.0 \\
7.5 \\
6.9 \\
8.2\end{array}$ \\
\hline 2009 & March & $14.5^{p}$ & $11.4^{p}$ & $7.4^{p}$ & $512^{p}$ & $3.2^{p}$ & $12.1^{p}$ & $0.05^{p}$ & $0.2^{p}$ & $2.5^{p}$ & $9.5^{p}$ & $14.8^{p}$ & $11.6^{p}$ & $0.06^{p}$ & $4.3^{p}$ & $0.04^{p}$ & $2.9^{p}$ & $0.10^{p}$ & $6.7^{p}$ \\
\hline $\begin{array}{l}\text { North } \\
1976 \\
1981 \\
1986 \\
1991 \\
1996\end{array}$ & thern Ireland & $\begin{array}{l}26.4 \\
27.2 \\
28.0 \\
26.0 \\
24.4\end{array}$ & $\begin{array}{l}17.3 \\
17.6 \\
17.8 \\
16.2 \\
14.7\end{array}$ & $\begin{array}{l}1.3 \\
1.9 \\
3.6 \\
5.3 \\
6.3\end{array}$ & $\begin{array}{r}50 \\
70 \\
128 \\
203 \\
260\end{array}$ & $\begin{array}{r}9.9 \\
9.6 \\
10.2 \\
9.2 \\
8.3\end{array}$ & $\begin{array}{r}45.4 \\
. . \\
. .\end{array}$ & $\begin{array}{l}\vdots \\
\vdots \\
\vdots\end{array}$ & $\vdots$ & $\begin{array}{l}0.6 \\
1.4 \\
1.5 \\
2.3 \\
2.3\end{array}$ & $\begin{array}{l}4.2 \\
. . \\
. . \\
. .\end{array}$ & $\begin{array}{l}17.0 \\
16.3 \\
16.1 \\
15.1 \\
15.2\end{array}$ & $\begin{array}{r}11.2 \\
10.6 \\
10.3 \\
9.4 \\
9.2\end{array}$ & $\begin{array}{l}0.48 \\
0.36 \\
0.36 \\
0.19 \\
0.14\end{array}$ & $\begin{array}{r}18.3 \\
13.2 \\
13.2 \\
7.4 \\
5.8\end{array}$ & $\begin{array}{l}0.35 \\
0.23 \\
0.23 \\
0.12 \\
0.09\end{array}$ & $\begin{array}{r}13.3 \\
8.3 \\
8.3 \\
4.6 \\
3.7\end{array}$ & $\begin{array}{l}0.59 \\
0.42 \\
0.42 \\
0.22 \\
0.23\end{array}$ & $\begin{array}{r}22.3 \\
15.3 \\
15.3 \\
8.4 \\
9.4\end{array}$ \\
\hline $\begin{array}{l}1999 \\
2000 \\
2001 \\
2002 \\
2003\end{array}$ & & $\begin{array}{l}23.0 \\
21.5 \\
22.0 \\
21.4 \\
21.6\end{array}$ & $\begin{array}{l}13.7 \\
12.8 \\
13.0 \\
12.6 \\
12.7\end{array}$ & $\begin{array}{l}7.0 \\
6.8 \\
7.1 \\
7.2 \\
7.4\end{array}$ & $\begin{array}{l}303 \\
318 \\
325 \\
335 \\
344\end{array}$ & $\begin{array}{l}7.6 \\
7.6 \\
77.3 \\
7.6 \\
7.8\end{array}$ & $\begin{array}{l}. . \\
. . \\
. . \\
. .\end{array}$ & $\vdots$ & $\vdots$ & $\begin{array}{l}2.3 \\
2.4 \\
2.4 \\
2.2 \\
2.3\end{array}$ & $\begin{array}{l}. . \\
. . \\
. . \\
. .\end{array}$ & $\begin{array}{l}15.7 \\
14.9 \\
14.5 \\
14.6 \\
14.5\end{array}$ & $\begin{array}{l}9.3 \\
8.9 \\
8.6 \\
8.6 \\
8.5\end{array}$ & $\begin{array}{l}0.15 \\
0.11 \\
0.13 \\
0.10 \\
0.12\end{array}$ & $\begin{array}{l}6.4 \\
5.0 \\
6.0 \\
4.6 \\
5.2\end{array}$ & $\begin{array}{l}0.11 \\
0.08 \\
0.10 \\
0.07 \\
0.09\end{array}$ & $\begin{array}{l}4.8 \\
3.8 \\
4.5 \\
3.5 \\
4.0\end{array}$ & $\begin{array}{l}0.23 \\
0.16 \\
0.19 \\
0.19 \\
0.18\end{array}$ & $\begin{array}{r}10.0 \\
7.2 \\
8.4 \\
8.7 \\
8.0\end{array}$ \\
\hline $\begin{array}{l}2004 \\
2005 \\
2006 \\
2007 \\
2008\end{array}$ & & $\begin{array}{l}22.3 \\
22.3 \\
23.3 \\
24.5 \\
25.6^{p}\end{array}$ & $\begin{array}{l}13.0 \\
12.9 \\
13.4 \\
13.9 \\
14.4^{\mathrm{p}}\end{array}$ & $\begin{array}{c}7.7 \\
8.1 \\
8.8 \\
9.3 \\
10.0^{\mathrm{p}}\end{array}$ & $\begin{array}{l}345 \\
363 \\
380 \\
379 \\
389^{p}\end{array}$ & $\begin{array}{l}8.3 \\
8.1 \\
8.3 \\
8.7 \\
8.5^{p}\end{array}$ & $\begin{array}{l}. . \\
. . \\
. . \\
. .\end{array}$ & $\begin{array}{l}0.0110 \\
0.12 \\
0.11 \\
0.09^{p}\end{array}$ & $\begin{array}{l}: \\
: .\end{array}$ & $\begin{array}{l}2.5 \\
2.4 \\
2.6 \\
2.9^{p} \\
2.8^{p}\end{array}$ & $\begin{array}{l}. . \\
. . \\
. . \\
. .\end{array}$ & $\begin{array}{l}14.4 \\
14.2 \\
14.5 \\
14.6 \\
14.9^{p}\end{array}$ & $\begin{array}{l}8.4 \\
8.3 \\
8.4 \\
8.3 \\
8.4^{\mathrm{p}}\end{array}$ & $\begin{array}{l}0.12 \\
0.14 \\
0.12 \\
0.12 \\
0.12^{\mathrm{p}}\end{array}$ & $\begin{array}{l}5.3 \\
6.1 \\
5.1 \\
4.9 \\
4.7^{p}\end{array}$ & $\begin{array}{l}0.08 \\
0.11 \\
0.09 \\
0.08 \\
0.10^{p}\end{array}$ & $\begin{array}{l}3.7 \\
4.9 \\
3.8 \\
3.2 \\
3.6^{p}\end{array}$ & $\begin{array}{l}0.19 \\
0.19 \\
0.17 \\
0.17 \\
0.19^{p}\end{array}$ & $\begin{array}{l}8.0 \\
8.1 \\
6.9 \\
6.9 \\
7.4^{\mathrm{p}}\end{array}$ \\
\hline 2007 & $\begin{array}{l}\text { March } \\
\text { June } \\
\text { Sept } \\
\text { Dec }\end{array}$ & $\begin{array}{l}6.1 \\
6.0 \\
6.5 \\
5.9\end{array}$ & $\begin{array}{l}14.2 \\
13.7 \\
14.5 \\
13.2\end{array}$ & $\begin{array}{l}2.4 \\
2.2 \\
2.5 \\
2.2\end{array}$ & $\begin{array}{l}383 \\
365 \\
386 \\
380\end{array}$ & $\begin{array}{l}1.0 \\
2.4 \\
3.8 \\
1.6\end{array}$ & $\begin{array}{l}. . \\
. . \\
. .\end{array}$ & $\begin{array}{l}0.02 \\
0.03 \\
0.04 \\
0.02\end{array}$ & .. & $\begin{array}{l}0.9 \\
0.7 \\
0.7 \\
0.6\end{array}$ & $\begin{array}{l}. . \\
. . \\
.\end{array}$ & $\begin{array}{l}4.2 \\
3.6 \\
3.3 \\
3.6\end{array}$ & $\begin{array}{l}9.7 \\
8.2 \\
7.3 \\
8.1\end{array}$ & $\begin{array}{l}0.04 \\
0.03 \\
0.03 \\
0.02\end{array}$ & $\begin{array}{l}6.7 \\
5.1 \\
4.6 \\
3.3\end{array}$ & $\begin{array}{l}0.03 \\
0.02 \\
0.02 \\
0.01\end{array}$ & $\begin{array}{l}4.6 \\
2.9 \\
3.2 \\
2.2\end{array}$ & $\begin{array}{l}0.05 \\
0.04 \\
0.05 \\
0.04\end{array}$ & $\begin{array}{l}7.6 \\
6.7 \\
7.0 \\
6.5\end{array}$ \\
\hline 2008 & $\begin{array}{l}3 \text { March } \\
\text { June } \\
\text { Sept } \\
\text { Dec }\end{array}$ & $\begin{array}{l}6.5^{p} \\
6.3^{p} \\
6.5^{p} \\
6.3^{p}\end{array}$ & $\begin{array}{l}14.8^{p} \\
143^{p} \\
14.4^{p} \\
14.1^{p}\end{array}$ & $\begin{array}{l}2.4^{p} \\
2.4^{p} \\
2.5^{p} \\
2.5^{p}\end{array}$ & $\begin{array}{l}396^{\mathrm{p}} \\
384^{\mathrm{p}} \\
366^{\mathrm{p}} \\
389^{\mathrm{p}}\end{array}$ & $\begin{array}{l}1.1^{p} \\
2.2^{p} \\
3.6^{p} \\
1.6^{p}\end{array}$ & $\begin{array}{l}. . \\
. . \\
. .\end{array}$ & $\begin{array}{l}0.02^{\mathrm{p}} \\
0.02^{\mathrm{p}} \\
0.02^{\mathrm{p}} \\
0.02^{\mathrm{p}}\end{array}$ & $\begin{array}{l}. . \\
. . \\
.\end{array}$ & $\begin{array}{l}0.8^{p} \\
0.7^{p} \\
0.6^{p} \\
0.7^{p}\end{array}$ & $\begin{array}{l}. . \\
. . \\
. .\end{array}$ & $\begin{array}{l}4.1^{p} \\
3.6^{p} \\
3.4^{p} \\
3.7^{p}\end{array}$ & $\begin{array}{l}9.4^{p} \\
8.2^{p} \\
7.7^{p} \\
8.4^{p}\end{array}$ & $\begin{array}{l}0.03^{\mathrm{P}} \\
0.03^{\mathrm{P}} \\
0.0 \mathrm{P}^{\mathrm{P}} \\
0.04^{\mathrm{P}}\end{array}$ & $\begin{array}{l}4.6^{\mathrm{p}} \\
4.6^{\mathrm{p}} \\
3.8^{\mathrm{p}} \\
5.7^{\mathrm{p}}\end{array}$ & $\begin{array}{l}0.03^{\mathrm{p}} \\
0.02^{\mathrm{p}} \\
0.02^{\mathrm{p}} \\
0.03^{\mathrm{p}}\end{array}$ & $\begin{array}{l}3.9^{p} \\
3.5^{p} \\
2.7^{p} \\
4.3^{p}\end{array}$ & $\begin{array}{l}0.05^{p} \\
0.04^{p} \\
0.05^{p} \\
0.055^{p}\end{array}$ & $\begin{array}{l}7.3^{p} \\
6.4^{p} \\
7.5^{p} \\
8.2^{p}\end{array}$ \\
\hline 2009 & March & $6.3^{p}$ & $14.3^{p}$ & $2.6^{\mathrm{p}}$ & $409^{\mathrm{P}}$ & $0.9^{p}$ & .. & $0.01^{\mathrm{P}}$ & .. & .. & .. & $4.2^{p}$ & $9.5^{p}$ & $0.04^{p}$ & $6.5^{p}$ & $0.03^{p}$ & $4.8^{p}$ & $0.07^{\mathrm{p}}$ & $10.0^{p}$ \\
\hline
\end{tabular}

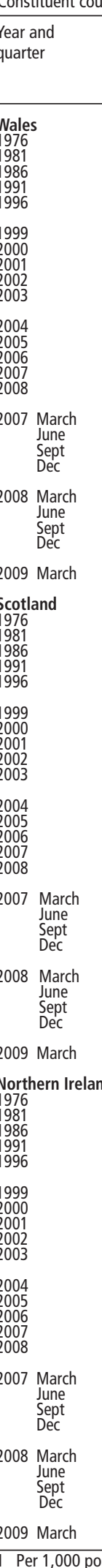

2 Per 1,000 live births.

4 Persons forming a civil partnership per 1,000 unmarried population aged 16 and over.

5 Persons divorcing per 1,000 married population.

6 Deaths under 1 year.

7 Deaths under 4 weeks.

Stillbirths and deaths under 1 week.

$\begin{aligned} & 9 \text { Per 1,000 live births and stillbirths. } \\ & 10 \text { The Civil Partnership Act } 2004 \text { came into force on } 5 \text { December } 2005 \text { in the UK - see Notes to }\end{aligned}$

p provisional 


\begin{tabular}{|c|c|c|c|c|c|c|c|c|c|c|c|c|c|c|c|c|c|}
\hline \multirow[b]{2}{*}{$\begin{array}{l}\text { Year and } \\
\text { quarter }\end{array}$} & \multicolumn{7}{|c|}{ Age of mother at birth } & \multirow{2}{*}{$\begin{array}{l}\text { Mean } \\
\text { age }^{1} \\
\text { (years) }\end{array}$} & \multicolumn{7}{|c|}{ Age of mother at birth } & \multirow{2}{*}{$\begin{array}{c}\text { Mean } \\
\text { age }^{2} \\
\text { (years) }\end{array}$} & \multirow[t]{2}{*}{$\mathrm{TFR}^{3}$} \\
\hline & $\begin{array}{l}\text { All } \\
\text { ages }\end{array}$ & $\begin{array}{c}\text { Under } \\
20\end{array}$ & $20-24$ & $25-29$ & $30-34$ & $35-39$ & $\begin{array}{l}40 \text { and } \\
\text { over }\end{array}$ & & $\begin{array}{c}\text { All } \\
\text { ages }\end{array}$ & $\begin{array}{c}\text { Under } \\
20\end{array}$ & $20-24$ & $25-29$ & $30-34$ & $35-39$ & $\begin{array}{c}40 \text { and } \\
\text { over }\end{array}$ & & \\
\hline & \multicolumn{7}{|c|}{ Total live births (numbers) } & & \multicolumn{7}{|c|}{ Age-specific fertility rates ${ }^{4}$} & & \\
\hline 1961 & 811.3 & 59.8 & 249.8 & 248.5 & 152.3 & 77.5 & 23.3 & 27.6 & 89.2 & 37.3 & 172.6 & 176.9 & 103.1 & 48.1 & 15.0 & 27.4 & 2.77 \\
\hline 1964(max) & 876.0 & 76.7 & 276.1 & 270.7 & 153.5 & 75.4 & 23.6 & 27.2 & 92.9 & 42.5 & 181.6 & 187.3 & 107.7 & 49.8 & 13.7 & 27.3 & 2.93 \\
\hline 1966 & 849.8 & 86.7 & 285.8 & 253.7 & 136.4 & 67.0 & 20.1 & 26.8 & 90.5 & 47.7 & 176.0 & 174.0 & 97.3 & 45.3 & 12.5 & 27.1 & 2.75 \\
\hline 1971 & 783.2 & 82.6 & 285.7 & 247.2 & 109.6 & 45.2 & 12.7 & 26.2 & 83.5 & 50.6 & 152.9 & 153.2 & 77.1 & 32.8 & 8.7 & 26.6 & 2.37 \\
\hline 1976 & 584.3 & 57.9 & 182.2 & 220.7 & 90.8 & 26.1 & 6.5 & 26.4 & 60.4 & 32.2 & 109.3 & 118.7 & 57.2 & 18.6 & 4.8 & 26.5 & 1.71 \\
\hline 1977(min) & 569.3 & 54.5 & 174.5 & 207.9 & 100.8 & 25.5 & 6.0 & 26.5 & 58.1 & 29.4 & 103.7 & 117.5 & 58.6 & 18.2 & 4.4 & 26.6 & 1.66 \\
\hline 1981 & 634.5 & 56.6 & 194.5 & 215.8 & 126.6 & 34.2 & 6.9 & 26.8 & 61.3 & 28.1 & 105.3 & 129.1 & 68.6 & 21.7 & 4.9 & 27.0 & 1.79 \\
\hline 1986 & 661.0 & 57.4 & 192.1 & 229.0 & 129.5 & 45.5 & 7.6 & 27.0 & 60.6 & 30.1 & 92.7 & 123.8 & 78.0 & 24.6 & 4.8 & 27.4 & 1.77 \\
\hline 1991 & 699.2 & 52.4 & 173.4 & 248.7 & 161.3 & 53.6 & 9.8 & 27.7 & 63.6 & 33.0 & 89.3 & 119.4 & 86.7 & 32.1 & 5.3 & 27.7 & 1.82 \\
\hline 1992 & 689.7 & 47.9 & 163.3 & 244.8 & 166.8 & 56.7 & 10.2 & 27.9 & 63.6 & 31.7 & 86.1 & 117.6 & 87.4 & 33.4 & 5.8 & 27.8 & 1.80 \\
\hline 1993 & 673.5 & 45.1 & 152.0 & 236.0 & 171.1 & 58.8 & 10.5 & 28.1 & 62.7 & 30.9 & 82.5 & 114.4 & 87.4 & 34.1 & 6.2 & 27.9 & 1.76 \\
\hline 1994 & 664.7 & 42.0 & 140.2 & 229.1 & 179.6 & 63.1 & 10.7 & 28.4 & 62.0 & 28.9 & 79.0 & 112.2 & 89.4 & 35.8 & 6.4 & 28.1 & 1.75 \\
\hline 1995 & 648.1 & 41.9 & 130.7 & 217.4 & 181.2 & 65.5 & 11.3 & 28.5 & 60.5 & 28.5 & 76.4 & 108.4 & 88.3 & 36.3 & 6.8 & 28.2 & 1.72 \\
\hline 1996 & 649.5 & 44.7 & 125.7 & 211.1 & 186.4 & 69.5 & 12.1 & 28.6 & 60.6 & 29.7 & 77.0 & 106.6 & 89.8 & 37.5 & 7.2 & 28.2 & 1.74 \\
\hline 1997 & 643.1 & 46.4 & 118.6 & 202.8 & 187.5 & 74.9 & 12.9 & 28.8 & 60.0 & 30.2 & 76.0 & 104.3 & 89.8 & 39.4 & 7.6 & 28.3 & 1.73 \\
\hline 1998 & 635.9 & 48.3 & 113.5 & 193.1 & 188.5 & 78.9 & 13.6 & 28.9 & 59.2 & 30.9 & 74.9 & 101.5 & 90.6 & 40.4 & 7.9 & 28.3 & 1.72 \\
\hline 1999 & 621.9 & 48.4 & 110.7 & 181.9 & 185.3 & 81.3 & 14.3 & 29.0 & 57.8 & 30.9 & 73.0 & 98.3 & 89.6 & 40.6 & 8.1 & 28.4 & 1.70 \\
\hline 2000 & 604.4 & 45.8 & 107.7 & 170.7 & 180.1 & 85.0 & 15.1 & 29.1 & 55.9 & 29.3 & 70.0 & 94.3 & 87.9 & 41.4 & 8.3 & 28.5 & 1.65 \\
\hline 2001 & 594.6 & 44.2 & 108.8 & 159.9 & 178.9 & 86.5 & 16.3 & 29.2 & 54.7 & 28.0 & 69.0 & 91.7 & 88.0 & 41.5 & 8.8 & 28.6 & 1.63 \\
\hline 2002 & 596.1 & 43.5 & 110.9 & 153.4 & 180.5 & 90.5 & 17.3 & 29.3 & 54.7 & 27.1 & 69.1 & 91.5 & 89.9 & 43.0 & 9.1 & 28.7 & 1.65 \\
\hline 2003 & 621.5 & 44.2 & 116.6 & 156.9 & 187.2 & 97.4 & 19.1 & 29.4 & 56.8 & 26.9 & 71.3 & 95.8 & 94.9 & 46.4 & 9.8 & 28.8 & 1.73 \\
\hline 2004 & 639.7 & 45.1 & 121.1 & 160.0 & 190.6 & 102.2 & 20.8 & 29.4 & 58.2 & 26.9 & 72.8 & 97.6 & 99.6 & 48.8 & 10.4 & 28.9 & 1.78 \\
\hline 2005 & 645.8 & 44.8 & 122.1 & 164.3 & 188.2 & 104.1 & 22.2 & 29.5 & 58.3 & 26.3 & 71.6 & 97.9 & 100.7 & 50.3 & 10.8 & 29.1 & 1.79 \\
\hline 2006 & 669.6 & 45.5 & 127.8 & 172.6 & 189.4 & 110.5 & 23.7 & 29.5 & 60.2 & 26.6 & 73.2 & 100.6 & 104.8 & 53.8 & 11.4 & 29.1 & 1.86 \\
\hline 2007 & 690.0 & 44.8 & 130.8 & 182.6 & 191.1 & 115.4 & 25.4 & 29.5 & 62.0 & 26.0 & 73.5 & 104.0 & 110.2 & 56.9 & 12.0 & 29.3 & 1.92 \\
\hline 2008 & 708.7 & 44.7 & 136.0 & 193.0 & 192.5 & 116.2 & 26.4 & 29.5 & $63.5^{p}$ & $26.2^{p}$ & $74.3^{p}$ & $106.2^{p}$ & $112.3^{P}$ & $58.4^{p}$ & $12.6^{P}$ & $29.3^{p}$ & $1.95^{\mathrm{P}}$ \\
\hline 2004 March & 155.2 & 11.0 & 29.3 & 38.7 & 46.6 & 24.7 & 4.9 & 29.4 & 56.8 & 26.5 & 70.8 & 95.0 & 97.9 & 47.4 & 9.8 & 28.9 & 1.74 \\
\hline June & 157.4 & 10.7 & 29.3 & 39.4 & 47.7 & 25.2 & 5.0 & 29.5 & 57.6 & 25.7 & 70.9 & 96.6 & 100.4 & 48.5 & 10.1 & 29.0 & 1.76 \\
\hline Sept & 165.4 & 11.7 & 31.4 & 41.6 & 49.0 & 26.3 & 5.4 & 29.4 & 59.9 & 27.7 & 75.0 & 101.0 & 102.0 & 50.1 & 10.7 & 28.9 & 1.83 \\
\hline Dec & 161.7 & 11.6 & 31.1 & 40.3 & 47.2 & 26.0 & 5.5 & 29.4 & 58.5 & 27.6 & 74.3 & 97.7 & 98.2 & 49.4 & 10.9 & 28.9 & 1.79 \\
\hline 2005 March & 154.3 & 10.9 & 29.3 & 38.9 & 45.0 & 24.7 & 5.4 & 29.4 & 56.5 & 26.0 & 69.6 & 94.0 & 97.6 & 48.5 & 10.7 & 29.0 & 1.74 \\
\hline June & 159.8 & 10.7 & 29.6 & 40.3 & 47.5 & 26.2 & 5.4 & 29.5 & 57.8 & 25.3 & 69.7 & 96.2 & 101.9 & 50.8 & 10.6 & 29.1 & 1.78 \\
\hline Sept & 170.2 & 11.9 & 32.5 & 43.7 & 49.4 & 26.9 & 5.7 & 29.4 & 60.9 & 27.6 & 75.7 & 103.2 & 104.9 & 51.6 & 11.1 & 29.0 & 1.88 \\
\hline Dec & 161.7 & 11.3 & 30.7 & 41.4 & 46.3 & 26.3 & 5.7 & 29.4 & 57.9 & 26.3 & 71.3 & 97.9 & 98.3 & 50.4 & 11.0 & 29.0 & 1.78 \\
\hline 2006 March & 159.5 & 11.1 & 30.5 & 40.7 & 45.3 & 26.3 & 5.6 & 29.5 & 58.2 & 26.3 & 70.9 & 96.1 & 101.6 & 52.0 & 11.0 & 29.1 & 1.79 \\
\hline June & 166.2 & 11.4 & 31.2 & 42.9 & 47.6 & 27.1 & 5.9 & 29.5 & 60.0 & 26.6 & 71.8 & 100.4 & 105.7 & 53.0 & 11.3 & 29.1 & 1.85 \\
\hline Sept & 174.9 & 12.0 & 33.5 & 45.6 & 49.0 & 28.9 & 6.0 & 29.4 & 62.4 & 27.7 & 76.1 & 105.4 & 107.5 & 55.9 & 11.4 & 29.1 & 1.93 \\
\hline Dec & 169.0 & 11.1 & 32.6 & 43.5 & 47.5 & 28.1 & 6.2 & 29.5 & 60.3 & 25.7 & 74.0 & 100.5 & 104.3 & 54.4 & 11.8 & 29.2 & 1.86 \\
\hline 2007 March & 164.0 & 10.9 & 31.1 & 42.7 & 45.7 & 27.4 & 6.2 & 29.5 & 59.8 & 25.5 & 70.9 & 98.6 & 106.9 & 54.8 & 12.0 & 29.3 & 1.85 \\
\hline June & 169.5 & 10.7 & 31.4 & 44.6 & 47.8 & 28.9 & 6.2 & 29.6 & 61.1 & 25.0 & 70.8 & 101.9 & 110.5 & 57.1 & 11.8 & 29.4 & 1.89 \\
\hline Sept & 181.4 & 11.9 & 34.6 & 48.6 & 50.0 & 29.9 & 6.4 & 29.5 & 64.7 & 27.3 & 77.1 & 109.9 & 114.4 & 58.6 & 12.0 & 29.2 & 2.00 \\
\hline Dec & 175.0 & 11.3 & 33.7 & 46.6 & 47.6 & 29.2 & 6.6 & 29.5 & 62.4 & 26.1 & 75.0 & 105.4 & 108.9 & 57.1 & 12.4 & 29.3 & 1.93 \\
\hline 2008 March & 173.8 & 11.1 & 33.5 & 46.7 & 47.2 & 28.8 & 6.4 & 29.5 & $62.6^{p}$ & $26.2^{p}$ & $73.8^{p}$ & $103.3^{P}$ & $110.7^{\mathrm{P}}$ & $58.2^{p}$ & $12.3^{P}$ & $29.3^{P}$ & $1.92^{\mathrm{P}}$ \\
\hline June & 177.0 & 11.0 & 33.5 & 48.3 & 48.4 & 29.2 & 6.6 & 29.5 & $63.8^{p}$ & $25.9^{p}$ & $73.6^{P}$ & $107.0^{\mathrm{p}}$ & $113.5^{P}$ & $59.0^{p}$ & $12.6^{p}$ & $29.4^{\mathrm{P}}$ & $1.96^{\mathrm{P}}$ \\
\hline Sept & 182.4 & 11.4 & 35.2 & 50.3 & 49.3 & 29.5 & 6.7 & 29.4 & $65.0^{p}$ & $26.7^{p}$ & $76.5^{p}$ & $110.1^{\mathrm{p}}$ & $114.4^{\mathrm{p}}$ & $59.0^{\mathrm{P}}$ & $12.8^{p}$ & $29.3^{p}$ & $2.00^{\mathrm{P}}$ \\
\hline Dec & 175.5 & 11.1 & 33.8 & 47.7 & 47.6 & 28.7 & 6.7 & 29.5 & $62.6^{p}$ & $26.0^{p}$ & $73.5^{p}$ & $104.3^{p}$ & $110.6^{P}$ & $57.3^{P}$ & $12.6^{P}$ & $29.3^{p}$ & $1.92^{\mathrm{p}}$ \\
\hline 2009 March & $168.1^{\mathrm{P}}$ & $10.9^{p}$ & $32.7^{p}$ & $45.9^{P}$ & $45.1^{\mathrm{P}}$ & $27.1^{\mathrm{P}}$ & $6.5^{p}$ & $29.4^{p}$ & $61.1^{p}$ & $26.1^{\mathrm{P}}$ & $71.4^{\mathrm{P}}$ & $99.7^{\mathrm{P}}$ & $106.4^{\mathrm{P}}$ & $56.7^{P}$ & $12.6^{p}$ & $29.3^{p}$ & $1.86^{\mathrm{P}}$ \\
\hline
\end{tabular}

Note: The rates for women of all ages, under 20 , and 40 and over are based upon the populations of women aged 15-44, 15-19, and 40-44 respectively. Rates for 2008 and 2009 are based on 2006-based population projections for 2008 and 2009.

1 Unstandardised and therefore takes no account of the age structure of the population.

2 Standardised to take account of the age structure of the population. This measure is more appropriate for use when analysing trends or making comparisons between different geographies.

3 TFR (total fertility rate) is the number of children that would be born to a woman if current patterns of fertility persisted throughout her childbearing life. It is sometimes called the TPFR (total period fertility rate).

4 Births per 1,000 women in the age-group; all quarterly age-specific fertility rates are adjusted for days in the quarter. They are not adjusted for seasonality.

p provisional. 
Numbers (thousands), mean age and percentages

\begin{tabular}{|c|c|c|c|c|c|c|c|c|c|c|c|c|c|c|c|c|c|c|}
\hline \multirow{3}{*}{$\begin{array}{l}\text { Year and } \\
\text { quarter }\end{array}$} & \multicolumn{7}{|c|}{ Age of mother at birth } & & \multicolumn{7}{|c|}{ Age of mother at birth } & \multicolumn{3}{|c|}{ Registration ${ }^{2}$} \\
\hline & All & Under & $20-24$ & $25-29$ & $30-34$ & $35-39$ & 40 and & \multirow{3}{*}{$\begin{array}{c}\text { Mean } \\
\text { age }^{1} \\
\text { (years) }\end{array}$} & \multirow{2}{*}{$\begin{array}{l}\text { All } \\
\text { ages }\end{array}$} & \multirow{2}{*}{$\begin{array}{l}\text { Under } \\
20\end{array}$} & \multirow[t]{2}{*}{$20-24$} & \multirow[t]{2}{*}{$25-29$} & \multirow[t]{2}{*}{$30-34$} & \multirow[t]{2}{*}{$35-39$} & \multirow{2}{*}{$\begin{array}{c}40 \text { and } \\
\text { over }\end{array}$} & \multicolumn{2}{|c|}{ Joint } & \multirow[t]{2}{*}{ Sole } \\
\hline & & & & & & & & & & & & & & & & $\begin{array}{c}\text { Same }^{3} \\
\text { address }\end{array}$ & $\begin{array}{l}\text { Different }^{3} \\
\text { address }\end{array}$ & \\
\hline & \multicolumn{7}{|c|}{ Live births outside marriage (numbers) } & & \multicolumn{7}{|c|}{$\begin{array}{l}\text { Percentage of total live births } \\
\text { in age group }\end{array}$} & \multicolumn{3}{|c|}{$\begin{array}{l}\text { As a percentage of all } \\
\text { births outside marriage }\end{array}$} \\
\hline
\end{tabular}

\begin{tabular}{|c|c|c|c|c|c|c|c|c|c|c|c|c|c|c|c|c|c|c|c|}
\hline 1971 & & 65.7 & 21.6 & 22.0 & 11.5 & 6.2 & 3.2 & 1.1 & 23.7 & 8.4 & 26.1 & 7.7 & 4.7 & 5.7 & 7.0 & 9.0 & \multicolumn{2}{|c|}{45.5} & 54.5 \\
\hline 1976 & & 53.8 & 19.8 & 16.6 & 9.7 & 4.7 & 2.3 & 0.7 & 23.3 & 9.2 & 34.2 & 9.1 & 4.4 & 5.2 & 8.6 & 10.1 & \multirow{2}{*}{\multicolumn{2}{|c|}{$\begin{array}{l}51.0 \\
58.2\end{array}$}} & 49.0 \\
\hline 1981 & & 81.0 & 26.4 & 28.8 & 14.3 & 7.9 & 1.3 & 0.9 & 23.4 & 12.8 & 46.7 & 14.8 & 6.6 & 6.2 & 3.9 & 12.5 & & & 41.8 \\
\hline $\begin{array}{l}1986 \\
1991\end{array}$ & & $\begin{array}{l}141.3 \\
211.3\end{array}$ & $\begin{array}{l}39.6 \\
43.4\end{array}$ & $\begin{array}{l}54.1 \\
77.8\end{array}$ & $\begin{array}{l}27.7 \\
52.4\end{array}$ & $\begin{array}{l}13.1 \\
25.7\end{array}$ & $\begin{array}{l}5.7 \\
9.8\end{array}$ & $\begin{array}{l}1.1 \\
2.1\end{array}$ & $\begin{array}{l}23.8 \\
24.8\end{array}$ & $\begin{array}{l}21.4 \\
30.2\end{array}$ & $\begin{array}{l}69.0 \\
82.9\end{array}$ & $\begin{array}{l}28.2 \\
44.9\end{array}$ & $\begin{array}{l}12.1 \\
21.1\end{array}$ & $\begin{array}{l}10.1 \\
16.0\end{array}$ & $\begin{array}{l}12.6 \\
18.3\end{array}$ & $\begin{array}{l}14.7 \\
21.3\end{array}$ & $\begin{array}{l}46.6 \\
54.6\end{array}$ & $\begin{array}{l}19.6 \\
19.8\end{array}$ & $\begin{array}{l}33.8 \\
25.6\end{array}$ \\
\hline 1992 & & 215.2 & 40.1 & 77.1 & 55.9 & 28.9 & 10.9 & 2.3 & 25.2 & 31.2 & 83.7 & 47.2 & 22.8 & 17.3 & 19.3 & 22.9 & 55.4 & 20.7 & 23.9 \\
\hline 1993 & & 216.5 & 38.2 & 75.0 & 57.5 & 31.4 & 11.9 & 2.5 & 25.5 & 32.2 & 84.8 & 49.4 & 24.4 & 18.4 & 20.2 & 23.5 & 54.8 & 22.0 & 23.2 \\
\hline 1994 & & 215.5 & 35.9 & 71.0 & 58.5 & 34.0 & 13.4 & 2.7 & 25.8 & 32.4 & 85.5 & 50.6 & 25.5 & 18.9 & 21.2 & 25.2 & 57.5 & 19.8 & 22.7 \\
\hline 1995 & & 219.9 & 36.3 & 69.7 & 59.6 & 37.0 & 14.4 & 3.0 & 26.0 & 33.9 & 86.6 & 53.3 & 27.4 & 20.4 & 22.0 & 26.2 & 58.1 & 20.1 & 21.8 \\
\hline 1996 & & 232.7 & 39.3 & 71.1 & 62.3 & 40.5 & 16.2 & 3.2 & 26.1 & 35.8 & 88.0 & 56.5 & 29.5 & 21.7 & 23.4 & 26.7 & 58.1 & 19.9 & 21.9 \\
\hline 1997 & & 238.2 & 41.1 & 69.5 & 63.4 & 42.2 & 18.2 & 3.7 & 26.2 & 37.0 & 88.7 & 58.6 & 31.3 & 22.5 & 24.3 & 28.6 & 59.5 & 19.3 & 21.2 \\
\hline 1998 & & 240.6 & 43.0 & 67.8 & 62.4 & 43.9 & 19.6 & 3.9 & 26.3 & 37.8 & 89.1 & 59.7 & 32.3 & 23.3 & 24.8 & 29.0 & 60.9 & 18.3 & 20.8 \\
\hline 1999 & & 241.9 & 43.0 & 67.5 & 61.2 & 45.0 & 20.8 & 4.3 & 26.4 & 38.9 & 89.0 & 61.0 & 33.6 & 24.3 & 25.6 & 30.2 & 61.8 & 18.2 & 19.9 \\
\hline 2000 & & 238.6 & 41.1 & 67.5 & 59.1 & 43.9 & 22.3 & 4.7 & 26.5 & 39.5 & 89.7 & 62.6 & 34.6 & 24.4 & 26.2 & 31.0 & 62.7 & 18.2 & 19.2 \\
\hline 2001 & & 238.1 & 39.5 & 68.1 & 56.8 & 45.2 & 23.3 & 5.1 & 26.7 & 40.0 & 89.5 & 62.6 & 35.5 & 25.3 & 26.9 & 31.6 & 63.2 & 18.4 & 18.4 \\
\hline 2002 & & 242.0 & 38.9 & 70.2 & 55.8 & 46.4 & 25.1 & 5.6 & 26.8 & 40.6 & 89.5 & 63.3 & 36.4 & 25.7 & 27.7 & 32.2 & 63.7 & 18.5 & 17.8 \\
\hline 2003 & & 257.2 & 39.9 & 75.7 & 58.2 & 49.2 & 27.8 & 6.4 & 26.9 & 41.4 & 90.2 & 64.9 & 37.1 & 26.3 & 28.5 & 33.3 & 63.5 & 19.0 & 17.4 \\
\hline 2004 & & 269.7 & 41.0 & 79.8 & 61.4 & 50.7 & 29.7 & 7.1 & 27.0 & 42.2 & 91.0 & 65.9 & 38.4 & 26.6 & 29.0 & 34.0 & 63.6 & 19.6 & 16.8 \\
\hline 2005 & & 276.5 & 41.2 & 82.1 & 64.4 & 50.8 & 30.3 & 7.7 & 27.0 & 42.8 & 91.8 & 67.2 & 39.2 & 27.0 & 29.1 & 34.8 & 63.5 & 20.2 & 16.3 \\
\hline 2006 & & 291.4 & 42.3 & 87.7 & 69.3 & 51.4 & 32.2 & 8.4 & 27.0 & 43.5 & 93.0 & 68.6 & 40.1 & 27.1 & 29.2 & 35.5 & 63.7 & 20.8 & 15.6 \\
\hline 2007 & & 305.6 & 41.7 & 91.9 & 76.0 & 53.0 & 34.0 & 9.0 & 27.1 & 44.3 & 93.1 & 70.3 & 41.6 & 27.7 & 29.5 & 35.5 & 65.0 & 20.1 & 15.0 \\
\hline 2008 & & 320.8 & 42.0 & 97.9 & 82.6 & 54.4 & 34.6 & 9.5 & 27.1 & 45.3 & 93.9 & 71.9 & 42.8 & 28.3 & 29.8 & 35.9 & 65.5 & 20.3 & 14.2 \\
\hline 2003 & March & 61.0 & 9.8 & 18.0 & 13.9 & 11.6 & 6.3 & 1.5 & 26.8 & 41.4 & 90.1 & 64.5 & 37.0 & 26.9 & 29.1 & 33.3 & 63.0 & 18.9 & 18.1 \\
\hline & June & 62.8 & 9.6 & 18.3 & 14.2 & 12.2 & 6.9 & 1.6 & 27.0 & 40.5 & 90.0 & 64.0 & 36.2 & 25.7 & 28.3 & 33.7 & 64.0 & 18.5 & 17.4 \\
\hline & Sept & 67.6 & 10.3 & 20.0 & 15.3 & 13.0 & 7.3 & 1.7 & 26.9 & 41.5 & 90.2 & 65.6 & 38.3 & 26.4 & 28.6 & 33.3 & 63.7 & 19.3 & 18.0 \\
\hline & Dec & 65.8 & 10.2 & 19.5 & 14.9 & 12.5 & 7.3 & 1.6 & 26.9 & 42.2 & 90.4 & 65.6 & 38.0 & 27.7 & 29.5 & 32.9 & 63.3 & 19.4 & 17.4 \\
\hline 2004 & 4 March & 65.2 & 10.1 & 19.3 & 14.8 & 12.5 & 7.0 & 1.7 & 26.9 & 42.0 & 91.2 & 65.8 & 38.2 & 26.8 & 28.2 & 34.3 & 63.1 & 19.4 & 17.4 \\
\hline & June & 65.2 & 9.8 & 19.1 & 14.9 & 12.5 & 7.3 & 1.7 & 27.0 & 41.4 & 91.0 & 65.1 & 37.7 & 26.2 & 28.8 & 34.5 & 63.9 & 19.5 & 16.6 \\
\hline & Sept & 70.2 & 10.7 & 20.7 & 16.1 & 13.0 & 7.9 & 1.8 & 27.0 & 42.4 & 91.2 & 66.1 & 38.6 & 26.5 & 30.0 & 33.5 & 63.7 & 19.7 & 16.6 \\
\hline & Dec & 69.1 & 10.6 & 20.7 & 15.7 & 12.7 & 7.5 & 1.9 & 26.9 & 42.7 & 90.6 & 66.6 & 39.0 & 27.0 & 29.0 & 33.9 & 63.6 & 19.8 & 16.6 \\
\hline 2005 & March & 66.3 & 10.1 & 19.6 & 15.2 & 12.2 & 7.3 & 1.9 & 27.0 & 43.0 & 92.0 & 67.0 & 39.0 & 27.1 & 29.6 & 35.2 & 63.1 & 20.3 & 16.6 \\
\hline & June & 66.6 & 9.8 & 19.7 & 15.4 & 12.5 & 7.4 & 1.8 & 27.0 & 41.7 & 91.2 & 66.5 & 38.2 & 26.4 & 28.1 & 33.5 & 63.7 & 19.8 & 16.5 \\
\hline & Sept & 73.7 & 10.9 & 22.1 & 17.3 & 13.4 & 7.9 & 2.1 & 26.9 & 43.3 & 92.0 & 68.0 & 39.6 & 27.2 & 29.3 & 35.7 & 63.7 & 20.3 & 16.0 \\
\hline & Dec & 69.9 & 10.4 & 20.7 & 16.5 & 12.6 & 7.7 & 2.0 & 27.0 & 43.2 & 92.1 & 67.4 & 39.8 & 27.3 & 29.5 & 34.8 & 63.5 & 20.3 & 16.2 \\
\hline 2006 & 5 March & 68.7 & 10.3 & 20.8 & 16.0 & 12.0 & 7.6 & 1.9 & 26.9 & 43.1 & 93.1 & 68.1 & 39.4 & 26.5 & 28.9 & 34.4 & 63.1 & 20.9 & 16.0 \\
\hline & June & 71.4 & 10.5 & 21.2 & 16.9 & 12.8 & 7.8 & 2.1 & 27.0 & 43.0 & 92.6 & 68.0 & 39.4 & 26.9 & 28.8 & 35.0 & 63.7 & 20.6 & 15.6 \\
\hline & Sept & 76.8 & 11.1 & 23.1 & 18.6 & 13.4 & 8.4 & 2.2 & 27.0 & 43.9 & 92.8 & 69.0 & 40.7 & 27.3 & 29.2 & 36.9 & 64.1 & 20.5 & 15.4 \\
\hline & Dec & 74.5 & 10.3 & 22.6 & 17.8 & 13.2 & 8.4 & 2.2 & 27.1 & 44.1 & 93.3 & 69.2 & 40.9 & 27.8 & 29.8 & 35.7 & 63.6 & 21.0 & 15.4 \\
\hline 2007 & 7 March & 72.5 & 10.2 & 21.7 & 17.6 & 12.6 & 8.2 & 2.2 & 27.1 & 44.2 & 93.5 & 69.8 & 41.3 & 27.5 & 29.8 & 35.1 & 64.0 & 20.5 & 15.5 \\
\hline & June & 73.5 & 9.9 & 21.8 & 18.3 & 13.0 & 8.3 & 2.2 & 27.1 & 43.4 & 92.6 & 69.5 & 41.0 & 27.2 & 28.8 & 35.2 & 65.1 & 19.9 & 14.9 \\
\hline & Sept & 80.8 & 11.1 & 24.4 & 20.4 & 13.9 & 8.8 & 2.2 & 27.0 & 44.5 & 93.2 & 70.5 & 41.9 & 27.8 & 29.6 & 35.0 & 65.2 & 20.1 & 14.7 \\
\hline & Dec & 78.7 & 10.6 & 24.0 & 19.7 & 13.5 & 8.7 & 2.4 & 27.1 & 45.0 & 93.1 & 71.3 & 42.2 & 28.3 & 29.6 & 36.5 & 65.3 & 19.9 & 14.8 \\
\hline 2008 & 8 March & 78.6 & 10.5 & 23.9 & 20.0 & 13.3 & 8.6 & 2.3 & 27.1 & 45.2 & 94.3 & 71.1 & 42.8 & 28.3 & 29.9 & 35.9 & 65.3 & 20.0 & 14.7 \\
\hline & June & 79.4 & 10.3 & 23.9 & 20.5 & 13.7 & 8.6 & 2.4 & 27.1 & 44.9 & 93.5 & 71.5 & 42.3 & 28.4 & 29.5 & 35.8 & 66.0 & 19.8 & 14.2 \\
\hline & Sept & 83.1 & 10.8 & 25.5 & 21.6 & 13.9 & 8.9 & 2.4 & 27.0 & 45.5 & 94.0 & 72.4 & 43.0 & 28.2 & 30.1 & 36.2 & 65.8 & 20.4 & 13.8 \\
\hline & Dec & 79.8 & 10.4 & 24.5 & 20.6 & 13.4 & 8.5 & 2.4 & 27.0 & 45.5 & 93.7 & 72.5 & 43.2 & 28.2 & 29.6 & 35.8 & 64.9 & 21.1 & 14.0 \\
\hline 2009 & 9 March & $77.3^{P}$ & $10.3^{p}$ & $23.8^{p}$ & $20.1^{p}$ & $12.9^{p}$ & $8.0^{p}$ & $2.3^{p}$ & $27.0^{\mathrm{P}}$ & $46.0^{p}$ & $94.3^{p}$ & $72.9^{p}$ & $43.9^{p}$ & $28.5^{\mathrm{P}}$ & $29.4^{p}$ & $35.0^{p}$ & $65.0^{\mathrm{p}}$ & $21.2^{p}$ & $13.8^{\mathrm{P}}$ \\
\hline
\end{tabular}

1 Unstandardised and therefore takes no account of the age structure of the population.

2 Births outside marriage can be registered by both the mother and father (joint) or by the mother alone (sole).

3 Usual address(es) of parents.

p provisional 


\begin{tabular}{|c|c|c|c|c|c|c|c|c|c|c|c|c|c|c|c|c|}
\hline \multirow[b]{2}{*}{$\begin{array}{l}\text { Year and } \\
\text { quarter }\end{array}$} & \multicolumn{7}{|c|}{ Age of mother at birth } & \multirow{2}{*}{$\begin{array}{c}\text { Mean } \\
\text { age }^{2} \\
\text { (years) }\end{array}$} & \multicolumn{7}{|c|}{ Age of mother at birth } & \multirow{2}{*}{$\begin{array}{c}\text { Mean } \\
\text { age }^{2} \\
\text { (years) }\end{array}$} \\
\hline & $\begin{array}{c}\text { All } \\
\text { ages }\end{array}$ & $\begin{array}{l}\text { Under } \\
20\end{array}$ & $20-24$ & $25-29$ & $30-34$ & $35-39$ & $\begin{array}{c}40 \text { and } \\
\text { over }\end{array}$ & & $\begin{array}{c}\text { All } \\
\text { ages }\end{array}$ & $\begin{array}{c}\text { Under } \\
20\end{array}$ & $20-24$ & $25-29$ & $30-34$ & $35-39$ & $\begin{array}{c}40 \text { and } \\
\text { over }\end{array}$ & \\
\hline & \multicolumn{8}{|c|}{ Live births within marriage } & \multicolumn{8}{|c|}{ Live births within marriage to remarried women } \\
\hline
\end{tabular}

1971
1976
1981
19
1991
1995
1996
1997
1998
1999
2000
2001
2002
2004
2005
2006
2008

$\begin{array}{lrrrrrrrrr}717.5 & 61.1 & 263.7 & 235.7 & 103.4 & 42.1 & 11.6 & 26.4 & 19.4 & 0.1 \\ 530.5 & 38.1 & 165.6 & 211.0 & 86.1 & 23.9 & 5.8 & 26.6 & 26.7 & 0.1 \\ 553.5 & 30.1 & 165.7 & 201.5 & 118.7 & 31.5 & 6.0 & 27.2 & 38.8 & 0.1 \\ 519.7 & 17.8 & 138.0 & 201.3 & 116.4 & 39.8 & 6.4 & 27.9 & 41.7 & 0.0 \\ 487.9 & 8.9 & 95.6 & 196.3 & 135.5 & 43.8 & 7.7 & 28.9 & 39.4 & 0.0 \\ 428.2 & 5.6 & 61.0 & 157.9 & 144.2 & 51.1 & 8.4 & 29.8 & 33.3 & 0.0 \\ 416.8 & 5.4 & 54.7 & 148.8 & 145.9 & 53.3 & 8.9 & 30.0 & 32.6 & 0.0 \\ 404.9 & 5.2 & 49.1 & 139.4 & 145.3 & 56.7 & 9.2 & 30.3 & 31.4 & 0.0 \\ 395.3 & 5.3 & 45.7 & 130.7 & 144.6 & 59.3 & 9.6 & 30.5 & 30.2 & 0.0 \\ 380.0 & 5.3 & 43.2 & 120.7 & 140.3 & 60.5 & 9.9 & 30.6 & 27.5 & 0.0\end{array}$

$\begin{array}{rrrrrr}2.1 & 6.6 & 6.1 & 3.4 & 1.1 & 33.1 \\ 2.9 & 10.5 & 8.7 & 3.6 & 1.0 & 30.4\end{array}$

986

$\begin{array}{lllll}65.8 & 4.7 & 40.3 & 111.6 & 136.2 \\ 5.5 & 4.6 & 40.7 & 103.1 & 133.7\end{array}$

354.1

$\begin{array}{rrrr}4.6 & 40.7 & 103.1 & 133.7 \\ 4.6 & 40.7 & 97.6 & 134 .\end{array}$

62.7
63.2
65.4

10.4
11.1
11.8

30.8
30.9

25.8
23.9
22.8

0.0
0.0
0.0

$\begin{array}{ll}65.6 & 12.7 \\ 72.6 & 13.7\end{array}$

\begin{tabular}{l|l}
31.2 & 22.8 \\
31.2 & 21.5
\end{tabular}

0.0
0.0
0.0

$\begin{array}{ll}3.6 & 13.4 \\ 2.6 & 13.2 \\ 1.6 & 10.8\end{array}$

$\begin{array}{lll}0.8 & 7.2 & 14.0 \\ 0.7 & 6.4 & 13.9\end{array}$

$\begin{array}{lll}0.6 & 5.8 & 13.1 \\ 0.6 & 5.1 & 12.4\end{array}$

0.4
0.4
0.4
0.3
0.3

4.3
3.7

3.7
3.1
2.7
2.4

10.4
9.5
8.9

\begin{tabular}{llll|l}
137.4 & 73.8 & 14.5 & 31.3 & 20.0 \\
138.0 & 78.3 & 15.3 & 31.4 & 18.7 \\
138.2 & 81.4 & 16.4 & 31.5 & 16.9 \\
138.1 & 81.6 & 16.9 & 31.5 & 15.0
\end{tabular}

$\begin{array}{lllll}369.3 & 3.7 & 40.0 & 100.0 & 137.4 \\ 378.2 & 3.2 & 40.1 & 103.3 & 138.0 \\ 384.5 & 3.1 & 38.9 & 106.6 & 138.2 \\ 387.9 & 2.7 & 38.2 & 110.4 & 138 .\end{array}$

$81.6 \quad 16.9$

0.0
0.0
0.0

$0.3 \quad 2.2$

8.9
8.4
7.7

$\begin{array}{ll}0.3 & 2.1 \\ 0.2 & 1.9 \\ 0.2 & 1.8\end{array}$

6.8
6.1
5.3
4.5

$\begin{array}{ll}4.0 & 31.5 \\ 4.0 & 31.5\end{array}$

$\begin{array}{lrrrrr} & 91.6 & 0.7 & 9.4 & 25.0 & 33 \\ \text { June } & 96.0 & 0.8 & 9.6 & 26.3 & 34 \\ \text { Sept } & 100.6 & 0.8 & 10.2 & 28.3 & 36.1 \\ \text { Dec } & 96.3 & 0.8 & 9.7 & 27.0 & 34.1\end{array}$

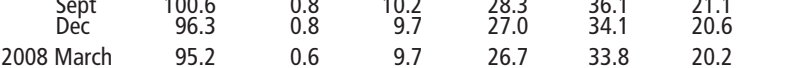

Sept
Dec

97.6

$\begin{array}{lll}0.6 & 9.7 & 26.7 \\ 0.7 & 9.6 & 27.9\end{array}$

$33.8 \quad 20.2$

31.5
31.4
31.5

0.2

$\begin{array}{llll}4.4 & 0.0 & 0.1 & 0.5 \\ 4.1 & 0.0 & 0.0 & 0.4 \\ 4.3 & 0.0 & 0.1 & 0.4 \\ 4.1 & 0.0 & 0.1 & 0.5\end{array}$

\begin{tabular}{ll|ll}
4.1 & 31.5 & 3.9 & 0.0
\end{tabular}

$0.1 \quad 0.5 \quad 1.3$

1.4
1.3
1.3
1.3

$\begin{array}{llllll}0.0 & 0.4 & 1.2 & 1.6 & 0.6 & 35.4 \\ 0.1 & 0.4 & 1.1 & 1.6 & 0.6 & 35.5\end{array}$

$\begin{array}{lllll}99.4 & 0.7 & 9.7 & 28.7 & 35.7 \\ 95.7 & 0.7 & 9.3 & 27.1 & 34.2\end{array}$

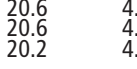

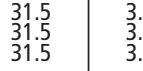

is

$0_{0.0}^{0.0}$

Second live births

\begin{tabular}{|c|c|c|c|c|c|c|c|c|c|c|c|c|c|c|c|c|}
\hline \multirow[b]{2}{*}{$\begin{array}{l}1971 \\
1976 \\
1981 \\
1986 \\
1991\end{array}$} & & \\
\hline & $\begin{array}{l}283.6 \\
217.2 \\
224.3 \\
206.9 \\
193.7\end{array}$ & $\begin{array}{r}49.5 \\
30.2 \\
23.6 \\
13.8 \\
6.7\end{array}$ & $\begin{array}{r}135.8 \\
85.4 \\
89.5 \\
74.7 \\
51.2\end{array}$ & $\begin{array}{l}74.8 \\
77.2 \\
77.2 \\
79.3 \\
84.5\end{array}$ & $\begin{array}{l}17.2 \\
19.7 \\
27.8 \\
30.8 \\
40.2\end{array}$ & $\begin{array}{l}5.1 \\
3.9 \\
5.4 \\
7.5 \\
9.7\end{array}$ & $\begin{array}{l}1.2 \\
0.7 \\
0.7 \\
0.9 \\
1.3\end{array}$ & $\begin{array}{l}23.9 \\
24.8 \\
25.3 \\
26.2 \\
27.5\end{array}$ & $\begin{array}{l}240.8 \\
203.6 \\
205.7 \\
189.2 \\
178.3\end{array}$ & $\begin{array}{r}10.7 \\
7.4 \\
6.1 \\
3.6 \\
2.0\end{array}$ & $\begin{array}{l}93.6 \\
62.5 \\
59.0 \\
47.5 \\
32.8\end{array}$ & $\begin{array}{l}94.1 \\
91.8 \\
82.7 \\
78.9 \\
73.9\end{array}$ & $\begin{array}{l}31.8 \\
34.7 \\
47.7 \\
45.5 \\
53.0\end{array}$ & $\begin{array}{r}8.9 \\
6.2 \\
9.1 \\
12.3 \\
14.7\end{array}$ & $\begin{array}{l}1.7 \\
1.0 \\
1.1 \\
1.3 \\
1.9\end{array}$ & $\begin{array}{l}26.2 \\
26.8 \\
27.4 \\
28.0 \\
28.9\end{array}$ \\
\hline $\begin{array}{l}1995 \\
1996 \\
1997 \\
1998 \\
1999\end{array}$ & $\begin{array}{l}168.1 \\
163.0 \\
157.0 \\
155.7 \\
153.4\end{array}$ & $\begin{array}{l}4.3 \\
4.2 \\
4.1 \\
4.2 \\
4.3\end{array}$ & $\begin{array}{l}32.3 \\
28.9 \\
25.9 \\
24.3 \\
23.5\end{array}$ & $\begin{array}{l}71.0 \\
67.2 \\
63.1 \\
60.6 \\
57.4\end{array}$ & $\begin{array}{l}46.6 \\
47.7 \\
48.1 \\
49.5 \\
50.0\end{array}$ & $\begin{array}{l}12.1 \\
13.1 \\
13.8 \\
15.0 \\
16.1\end{array}$ & $\begin{array}{l}1.8 \\
1.9 \\
2.0 \\
2.1 \\
2.2\end{array}$ & $\begin{array}{l}28.5 \\
28.8 \\
29.0 \\
29.2 \\
29.3\end{array}$ & $\begin{array}{l}158.1 \\
153.8 \\
150.4 \\
146.9 \\
139.5\end{array}$ & $\begin{array}{l}1.2 \\
1.0 \\
1.0 \\
1.0 \\
0.9\end{array}$ & $\begin{array}{l}20.6 \\
18.5 \\
16.6 \\
15.5 \\
14.4\end{array}$ & $\begin{array}{l}57.3 \\
53.4 \\
50.0 \\
46.4 \\
41.8\end{array}$ & $\begin{array}{l}58.5 \\
59.1 \\
59.4 \\
58.9 \\
56.6\end{array}$ & $\begin{array}{l}18.1 \\
19.2 \\
20.7 \\
22.2 \\
22.6\end{array}$ & $\begin{array}{l}2.4 \\
2.6 \\
2.7 \\
2.8 \\
3.1\end{array}$ & $\begin{array}{l}30.0 \\
30.3 \\
30.5 \\
30.7 \\
30.9\end{array}$ \\
\hline $\begin{array}{l}2000 \\
2001 \\
2002 \\
2003 \\
2004\end{array}$ & $\begin{array}{l}146.5 \\
143.9 \\
145.2 \\
151.0 \\
154.5\end{array}$ & $\begin{array}{l}3.8 \\
3.8 \\
3.8 \\
3.5 \\
3.3\end{array}$ & $\begin{array}{l}21.6 \\
22.2 \\
22.4 \\
22.2 \\
22.6\end{array}$ & $\begin{array}{l}52.7 \\
48.8 \\
47.1 \\
48.4 \\
48.9\end{array}$ & $\begin{array}{l}49.4 \\
49.7 \\
51.0 \\
54.2 \\
55.5\end{array}$ & $\begin{array}{l}16.6 \\
16.8 \\
18.1 \\
19.6 \\
20.7\end{array}$ & $\begin{array}{l}2.4 \\
2.6 \\
2.8 \\
3.1 \\
3.5\end{array}$ & $\begin{array}{l}29.6 \\
29.6 \\
29.8 \\
29.9 \\
30.0\end{array}$ & $\begin{array}{l}134.7 \\
132.2 \\
130.3 \\
132.9 \\
133.7\end{array}$ & $\begin{array}{l}0.8 \\
0.8 \\
0.7 \\
0.8 \\
0.7\end{array}$ & $\begin{array}{l}13.7 \\
13.7 \\
13.5 \\
13.9 \\
13.8\end{array}$ & $\begin{array}{l}38.4 \\
35.7 \\
33.0 \\
32.5 \\
31.9\end{array}$ & $\begin{array}{l}54.8 \\
53.8 \\
53.7 \\
54.3 \\
54.5\end{array}$ & $\begin{array}{l}23.8 \\
24.8 \\
25.6 \\
27.1 \\
28.3\end{array}$ & $\begin{array}{l}3.2 \\
3.5 \\
3.8 \\
4.2 \\
4.5\end{array}$ & $\begin{array}{l}31.1 \\
31.2 \\
31.4 \\
31.5 \\
31.6\end{array}$ \\
\hline $\begin{array}{l}2005 \\
2006 \\
2007 \\
2008\end{array}$ & $\begin{array}{l}156.0 \\
161.1 \\
165.6 \\
163.6\end{array}$ & $\begin{array}{l}3.0 \\
2.6 \\
2.5 \\
2.2\end{array}$ & $\begin{array}{l}22.1 \\
22.7 \\
22.2 \\
21.7\end{array}$ & $\begin{array}{l}50.0 \\
51.9 \\
54.4 \\
55.1\end{array}$ & $\begin{array}{l}55.7 \\
56.4 \\
57.2 \\
56.1\end{array}$ & $\begin{array}{l}21.4 \\
23.4 \\
25.0 \\
23.9\end{array}$ & $\begin{array}{l}3.8 \\
4.0 \\
4.5 \\
4.5\end{array}$ & $\begin{array}{l}30.1 \\
30.2 \\
30.3 \\
30.3\end{array}$ & $\begin{array}{l}132.0 \\
134.5 \\
135.2 \\
138.4\end{array}$ & $\begin{array}{l}0.6 \\
0.5 \\
0.5 \\
0.4\end{array}$ & $\begin{array}{l}13.2 \\
12.8 \\
12.2 \\
12.3\end{array}$ & $\begin{array}{l}32.1 \\
32.8 \\
33.6 \\
35.2\end{array}$ & $\begin{array}{l}52.8 \\
52.8 \\
52.1 \\
52.6\end{array}$ & $\begin{array}{l}28.6 \\
30.5 \\
31.5 \\
32.3\end{array}$ & $\begin{array}{l}4.8 \\
5.0 \\
5.4 \\
5.6\end{array}$ & $\begin{array}{l}31.7 \\
31.8 \\
31.9 \\
31.9\end{array}$ \\
\hline $\begin{array}{c}2007 \text { March } \\
\text { June } \\
\text { Sept } \\
\text { Dec }\end{array}$ & $\begin{array}{l}40.1 \\
40.7 \\
43.0 \\
41.9\end{array}$ & $\begin{array}{l}0.6 \\
0.6 \\
0.7 \\
0.6\end{array}$ & $\begin{array}{l}5.5 \\
5.4 \\
5.7 \\
5.6\end{array}$ & $\begin{array}{l}12.8 \\
13.4 \\
14.3 \\
13.9\end{array}$ & $\begin{array}{l}14.0 \\
14.0 \\
14.9 \\
14.2\end{array}$ & $\begin{array}{l}6.1 \\
6.2 \\
6.3 \\
6.4\end{array}$ & $\begin{array}{l}1.1 \\
1.1 \\
1.1 \\
1.2\end{array}$ & $\begin{array}{l}30.4 \\
30.3 \\
30.3 \\
30.3\end{array}$ & $\begin{array}{l}31.7 \\
34.7 \\
35.5 \\
33.4\end{array}$ & $\begin{array}{l}0.1 \\
0.1 \\
0.1 \\
0.1\end{array}$ & $\begin{array}{l}2.9 \\
3.1 \\
3.3 \\
3.0\end{array}$ & $\begin{array}{l}7.8 \\
8.5 \\
8.9 \\
8.4\end{array}$ & $\begin{array}{l}12.3 \\
13.5 \\
13.6 \\
12.7\end{array}$ & $\begin{array}{l}7.3 \\
8.1 \\
8.2 \\
7.9\end{array}$ & $\begin{array}{l}1.3 \\
1.4 \\
1.3 \\
1.3\end{array}$ & $\begin{array}{l}31.9 \\
31.9 \\
31.8 \\
31.9\end{array}$ \\
\hline \multirow[t]{2}{*}{$\begin{array}{c}2008 \text { March } \\
\text { June } \\
\text { Sept } \\
\text { Dec }\end{array}$} & $\begin{array}{l}39.6 \\
40.7 \\
41.8 \\
41.4 \\
\end{array}$ & $\begin{array}{l}0.5 \\
0.6 \\
0.5 \\
0.6\end{array}$ & $\begin{array}{l}5.4 \\
5.4 \\
5.6 \\
5.3 \\
\end{array}$ & $\begin{array}{l}13.1 \\
13.9 \\
14.2 \\
13.8 \\
\end{array}$ & $\begin{array}{l}13.6 \\
13.7 \\
14.4 \\
14.4 \\
\end{array}$ & $\begin{array}{l}5.9 \\
5.9 \\
6.0 \\
6.1 \\
\end{array}$ & $\begin{array}{l}1.1 \\
1.1 \\
1.1 \\
1.2 \\
\end{array}$ & $\begin{array}{l}30.3 \\
30.3 \\
30.3 \\
30.4 \\
\end{array}$ & $\begin{array}{l}34.1 \\
35.3 \\
35.4 \\
33.5 \\
\end{array}$ & $\begin{array}{l}0.1 \\
0.1 \\
0.1 \\
0.1\end{array}$ & $\begin{array}{l}3.2 \\
3.1 \\
3.1 \\
3.0 \\
\end{array}$ & $\begin{array}{l}8.6 \\
9.0 \\
9.2 \\
8.4 \\
\end{array}$ & $\begin{array}{l}12.9 \\
13.5 \\
13.5 \\
12.7 \\
\end{array}$ & $\begin{array}{l}7.9 \\
8.3 \\
8.1 \\
8.0 \\
\end{array}$ & $\begin{array}{l}1.4 \\
1.4 \\
1.4 \\
1.4 \\
\end{array}$ & $\begin{array}{l}31.8 \\
31.9 \\
31.8 \\
32.0 \\
\end{array}$ \\
\hline & \multicolumn{8}{|c|}{ Third live births } & \multicolumn{8}{|c|}{ Fourth and higher order live births ${ }^{3}$} \\
\hline $\begin{array}{l}1971 \\
1976 \\
1981 \\
1986 \\
1991\end{array}$ & $\begin{array}{r}111.7 \\
71.0 \\
82.4 \\
80.8 \\
76.1\end{array}$ & $\begin{array}{l}0.9 \\
0.5 \\
0.4 \\
0.3 \\
0.2\end{array}$ & $\begin{array}{r}26.6 \\
14.4 \\
14.1 \\
12.7 \\
9.4\end{array}$ & $\begin{array}{l}43.6 \\
29.8 \\
29.5 \\
30.2 \\
26.8\end{array}$ & $\begin{array}{l}27.9 \\
19.5 \\
28.7 \\
25.6 \\
27.5\end{array}$ & $\begin{array}{r}10.4 \\
5.8 \\
8.7 \\
10.5 \\
10.5\end{array}$ & $\begin{array}{l}2.2 \\
1.1 \\
1.0 \\
1.5 \\
1.8\end{array}$ & $\begin{array}{l}28.7 \\
28.8 \\
29.5 \\
29.9 \\
30.4\end{array}$ & $\begin{array}{l}81.4 \\
38.8 \\
41.1 \\
42.7 \\
39.8\end{array}$ & $\begin{array}{l}0.1 \\
0.0 \\
0.0 \\
0.0 \\
0.0\end{array}$ & $\begin{array}{l}7.6 \\
3.3 \\
3.1 \\
3.1 \\
2.3\end{array}$ & $\begin{array}{l}23.2 \\
12.2 \\
12.0 \\
13.0 \\
11.1\end{array}$ & $\begin{array}{l}26.5 \\
12.1 \\
14.5 \\
14.5 \\
14.8\end{array}$ & $\begin{array}{r}17.6 \\
8.0 \\
8.3 \\
9.4 \\
8.9\end{array}$ & $\begin{array}{l}6.5 \\
3.1 \\
3.2 \\
2.8 \\
2.7\end{array}$ & $\begin{array}{l}30.7 \\
30.7 \\
31.1 \\
31.2 \\
31.6\end{array}$ \\
\hline $\begin{array}{l}1995 \\
1996 \\
1997 \\
1998 \\
1999\end{array}$ & $\begin{array}{l}66.7 \\
65.3 \\
63.2 \\
60.4 \\
56.4\end{array}$ & $\begin{array}{l}0.1 \\
0.1 \\
0.1 \\
0.1 \\
0.1\end{array}$ & $\begin{array}{l}6.5 \\
5.8 \\
5.3 \\
4.7 \\
4.2\end{array}$ & $\begin{array}{l}20.5 \\
19.6 \\
18.1 \\
16.4 \\
14.7\end{array}$ & $\begin{array}{l}26.1 \\
26.0 \\
25.1 \\
24.0 \\
22.3\end{array}$ & $\begin{array}{l}11.7 \\
12.0 \\
12.7 \\
13.1 \\
13.0\end{array}$ & $\begin{array}{l}1.8 \\
1.8 \\
2.0 \\
2.1 \\
2.1\end{array}$ & $\begin{array}{l}31.1 \\
31.3 \\
31.5 \\
31.8 \\
32.0\end{array}$ & $\begin{array}{l}35.3 \\
34.7 \\
34.2 \\
32.3 \\
30.7\end{array}$ & $\begin{array}{l}0.0 \\
0.0 \\
0.0 \\
0.0 \\
0.0\end{array}$ & $\begin{array}{l}1.6 \\
1.5 \\
1.4 \\
1.2 \\
1.1\end{array}$ & $\begin{array}{l}9.0 \\
8.6 \\
8.1 \\
7.4 \\
6.8\end{array}$ & $\begin{array}{l}13.1 \\
13.1 \\
12.7 \\
12.1 \\
11.4\end{array}$ & $\begin{array}{l}9.2 \\
9.0 \\
9.4 \\
9.0 \\
8.8\end{array}$ & $\begin{array}{l}2.4 \\
2.6 \\
2.6 \\
2.6 \\
2.6\end{array}$ & $\begin{array}{l}32.0 \\
32.2 \\
32.4 \\
32.6 \\
32.7\end{array}$ \\
\hline $\begin{array}{l}2000 \\
2001 \\
2002 \\
2003 \\
2004\end{array}$ & $\begin{array}{l}54.9 \\
52.1 \\
50.3 \\
52.0 \\
52.5\end{array}$ & $\begin{array}{l}0.1 \\
0.1 \\
0.1 \\
0.1 \\
0.1\end{array}$ & $\begin{array}{l}4.0 \\
3.9 \\
3.9 \\
3.8 \\
4.0\end{array}$ & $\begin{array}{l}14.1 \\
12.8 \\
11.8 \\
12.1 \\
12.1\end{array}$ & $\begin{array}{l}21.1 \\
19.8 \\
19.0 \\
19.2 \\
19.3\end{array}$ & $\begin{array}{l}13.5 \\
13.2 \\
13.1 \\
14.1 \\
14.3\end{array}$ & $\begin{array}{l}2.2 \\
2.3 \\
2.4 \\
2.6 \\
2.7\end{array}$ & $\begin{array}{l}32.1 \\
32.2 \\
32.3 \\
32.5 \\
32.5\end{array}$ & $\begin{array}{l}29.7 \\
28.3 \\
28.2 \\
28.4 \\
29.3\end{array}$ & $\begin{array}{l}0.0 \\
0.0 \\
0.0 \\
0.0 \\
0.0\end{array}$ & $\begin{array}{l}1.0 \\
0.9 \\
0.9 \\
1.0 \\
0.9\end{array}$ & $\begin{array}{l}6.4 \\
5.9 \\
5.6 \\
5.7 \\
5.7\end{array}$ & $\begin{array}{l}10.9 \\
10.4 \\
10.3 \\
10.2 \\
10.5\end{array}$ & $\begin{array}{l}8.7 \\
8.4 \\
8.5 \\
8.8 \\
9.2\end{array}$ & $\begin{array}{l}2.7 \\
2.7 \\
2.8 \\
2.8 \\
2.9\end{array}$ & $\begin{array}{l}32.8 \\
33.0 \\
33.1 \\
33.1 \\
33.2\end{array}$ \\
\hline $\begin{array}{l}2005 \\
2006 \\
2007 \\
2008\end{array}$ & $\begin{array}{l}52.2 \\
53.0 \\
53.4 \\
54.8\end{array}$ & $\begin{array}{l}0.1 \\
0.1 \\
0.1 \\
0.0\end{array}$ & $\begin{array}{l}3.8 \\
3.7 \\
3.6 \\
3.4\end{array}$ & $\begin{array}{l}12.3 \\
12.8 \\
12.8 \\
13.7\end{array}$ & $\begin{array}{l}18.7 \\
18.5 \\
18.5 \\
18.8\end{array}$ & $\begin{array}{l}14.5 \\
15.0 \\
15.2 \\
15.5\end{array}$ & $\begin{array}{l}2.9 \\
3.1 \\
3.2 \\
3.3\end{array}$ & $\begin{array}{l}32.5 \\
32.6 \\
32.6 \\
32.6\end{array}$ & $\begin{array}{l}29.2 \\
29.6 \\
30.1 \\
31.2\end{array}$ & $\begin{array}{l}0.0 \\
0.0 \\
0.0 \\
0.0\end{array}$ & $\begin{array}{l}0.9 \\
0.9 \\
0.9 \\
0.8\end{array}$ & $\begin{array}{l}5.6 \\
5.9 \\
5.9 \\
6.3\end{array}$ & $\begin{array}{l}10.2 \\
10.3 \\
10.4 \\
10.6\end{array}$ & $\begin{array}{l}9.4 \\
9.4 \\
9.7 \\
9.9\end{array}$ & $\begin{array}{l}3.0 \\
3.1 \\
3.3 \\
3.6\end{array}$ & $\begin{array}{l}33.3 \\
33.2 \\
33.4 \\
33.4\end{array}$ \\
\hline $\begin{array}{l}2007 \text { March } \\
\text { June } \\
\text { Sept } \\
\text { Dec }\end{array}$ & $\begin{array}{l}12.6 \\
13.3 \\
14.3 \\
13.3\end{array}$ & $\begin{array}{l}0.0 \\
0.0 \\
0.0 \\
0.0\end{array}$ & $\begin{array}{l}0.9 \\
0.9 \\
1.0 \\
0.9\end{array}$ & $\begin{array}{l}3.0 \\
3.1 \\
3.4 \\
3.2\end{array}$ & $\begin{array}{l}4.4 \\
4.6 \\
4.9 \\
4.6\end{array}$ & $\begin{array}{l}3.5 \\
3.8 \\
4.1 \\
3.8\end{array}$ & $\begin{array}{l}0.8 \\
0.8 \\
0.8 \\
0.8\end{array}$ & $\begin{array}{l}32.6 \\
32.7 \\
32.6 \\
32.7\end{array}$ & $\begin{array}{l}7.2 \\
7.4 \\
7.9 \\
7.7\end{array}$ & $\begin{array}{l}0.0 \\
0.0 \\
0.0 \\
0.0\end{array}$ & $\begin{array}{l}0.2 \\
0.2 \\
0.2 \\
0.2\end{array}$ & $\begin{array}{l}1.4 \\
1.4 \\
1.5 \\
1.5\end{array}$ & $\begin{array}{l}2.5 \\
2.6 \\
2.7 \\
2.7\end{array}$ & $\begin{array}{l}2.3 \\
2.4 \\
2.6 \\
2.5\end{array}$ & $\begin{array}{l}0.8 \\
0.9 \\
0.9 \\
0.9\end{array}$ & $\begin{array}{l}33.3 \\
33.5 \\
33.3 \\
33.4\end{array}$ \\
\hline $\begin{array}{l}2008 \text { March } \\
\text { June } \\
\text { Sept } \\
\text { Dec }\end{array}$ & $\begin{array}{l}13.6 \\
14.0 \\
14.1 \\
13.1\end{array}$ & $\begin{array}{l}0.0 \\
0.0 \\
0.0 \\
0.0\end{array}$ & $\begin{array}{l}0.9 \\
0.9 \\
0.9 \\
0.8\end{array}$ & $\begin{array}{l}3.3 \\
3.5 \\
3.6 \\
3.3\end{array}$ & $\begin{array}{l}4.7 \\
4.8 \\
4.8 \\
4.5\end{array}$ & $\begin{array}{l}3.9 \\
4.0 \\
4.0 \\
3.7\end{array}$ & $\begin{array}{l}0.8 \\
0.8 \\
0.9 \\
0.8\end{array}$ & $\begin{array}{l}32.6 \\
32.7 \\
32.6 \\
32.6\end{array}$ & $\begin{array}{l}7.9 \\
7.5 \\
8.0 \\
7.7\end{array}$ & $\begin{array}{l}0.0 \\
0.0 \\
0.0 \\
0.0\end{array}$ & $\begin{array}{l}0.2 \\
0.2 \\
0.2 \\
0.2\end{array}$ & $\begin{array}{l}1.6 \\
1.5 \\
1.6 \\
1.6\end{array}$ & $\begin{array}{l}2.7 \\
2.6 \\
2.7 \\
2.6\end{array}$ & $\begin{array}{l}2.5 \\
2.4 \\
2.5 \\
2.5\end{array}$ & $\begin{array}{l}0.9 \\
0.9 \\
0.9 \\
0.9\end{array}$ & $\begin{array}{l}33.3 \\
33.4 \\
33.3 \\
33.4\end{array}$ \\
\hline
\end{tabular}

1 Birth order is based on all live births within marriage to the mother by her present or any former husband.

2 The mean ages shown in this table are unstandardised and therefore take no account of changes in the structure of the population by age, marital status or parity.

3 Mean age at birth refers to fourth live births only. 
England and Wales (residents)

Numbers (thousands) and rates; and percentage terminated by abortion

\begin{tabular}{|l|l|l|l|l|l|l|l|l|l|l|}
\cline { 2 - 7 } & \multicolumn{7}{|c|}{ Age of woman at conception } \\
\hline Year and quarter & All ages & Under 16 & Under 18 & Under 20 & $20-24$ & $25-29$ & $30-34$ & $35-39$ & 40 and over \\
\hline
\end{tabular}

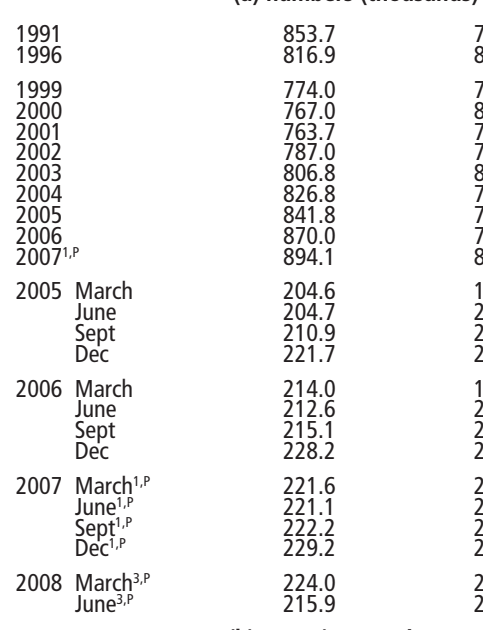

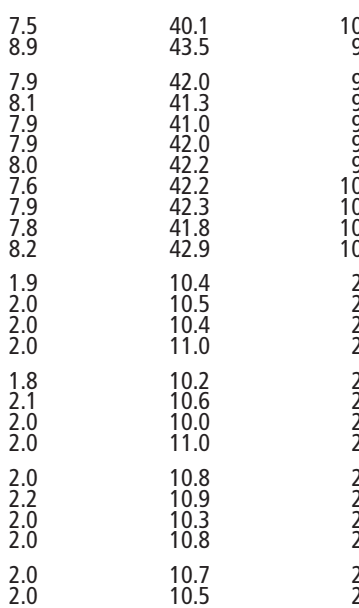

(b) rates (conceptions per thousand women in age group)

\begin{tabular}{|c|c|c|c|c|}
\hline $\begin{array}{l}1991 \\
1996\end{array}$ & & $\begin{array}{l}77.7 \\
76.2\end{array}$ & $\begin{array}{l}8.9 \\
9.5\end{array}$ & \\
\hline $\begin{array}{l}1999 \\
2000 \\
2001 \\
2002 \\
2003 \\
2004 \\
2005 \\
2006 \\
2007\end{array}$ & & $\begin{array}{l}71.9 \\
70.9 \\
70.3 \\
72.2 \\
73.7 \\
75.2 \\
76.0 \\
78.3 \\
80.3\end{array}$ & $\begin{array}{l}8.3 \\
8.3 \\
8.0 \\
7.9 \\
7.9 \\
7.5 \\
7.8 \\
7.8 \\
8.3\end{array}$ & \\
\hline 2005 & $\begin{array}{l}\text { March } \\
\text { June } \\
\text { Sept } \\
\text { Dec }\end{array}$ & $\begin{array}{l}75.1 \\
74.2 \\
75.5 \\
79.3\end{array}$ & $\begin{array}{l}7.6 \\
8.0 \\
7.8 \\
7.9\end{array}$ & \\
\hline 2006 & $\begin{array}{l}\text { March } \\
\text { June } \\
\text { Sept } \\
\text { Dec }\end{array}$ & $\begin{array}{l}78.2 \\
76.7 \\
76.8 \\
81.4\end{array}$ & $\begin{array}{l}7.1 \\
8.2 \\
7.7 \\
8.1\end{array}$ & \\
\hline 2007 & $\begin{array}{l}\text { March }{ }^{1,2, P} \\
\text { June }^{1,2, \mathrm{P}} \\
\text { Sept }^{1,2, \mathrm{P}} \\
\operatorname{Dec}^{1,2, \mathrm{P}}\end{array}$ & $\begin{array}{l}80.8 \\
79.7 \\
79.2 \\
81.6\end{array}$ & $\begin{array}{l}8.1 \\
8.7 \\
8.2 \\
8.3\end{array}$ & \\
\hline 2008 & $\begin{array}{l}\text { March } 2,3, \mathrm{P} \\
\text { June } \mathrm{e}^{2,3, \mathrm{P}}\end{array}$ & $\begin{array}{l}80.8 \\
77.9\end{array}$ & $\begin{array}{l}8.1 \\
8.2\end{array}$ & \\
\hline
\end{tabular}

\begin{tabular}{|c|c|c|c|c|}
\hline $\begin{array}{l}1991 \\
1996\end{array}$ & & $\begin{array}{l}19.4 \\
20.8\end{array}$ & $\begin{array}{l}51.1 \\
49.2\end{array}$ & $\begin{array}{l}39.9 \\
40.0\end{array}$ \\
\hline $\begin{array}{l}1999 \\
2000 \\
2001 \\
2002 \\
2003 \\
2004 \\
2005 \\
2006 \\
2007\end{array}$ & & $\begin{array}{l}22.6 \\
22.7 \\
23.2 \\
22.5 \\
22.5 \\
22.4 \\
22.2 \\
22.3 \\
22.0\end{array}$ & $\begin{array}{l}52.6 \\
54.0 \\
55.8 \\
55.6 \\
57.4 \\
57.2 \\
57.1 \\
59.8 \\
61.4\end{array}$ & $\begin{array}{l}43.0 \\
44.2 \\
45.7 \\
45.3 \\
45.7 \\
45.6 \\
46.3 \\
48.4 \\
50.0\end{array}$ \\
\hline 2005 & $\begin{array}{l}\text { March } \\
\text { June } \\
\text { Sept } \\
\text { Dec }\end{array}$ & $\begin{array}{l}22.5 \\
22.7 \\
21.4 \\
22.2\end{array}$ & $\begin{array}{l}57.5 \\
57.0 \\
56.2 \\
57.5\end{array}$ & $\begin{array}{l}47.3 \\
45.8 \\
45.3 \\
46.9\end{array}$ \\
\hline 2006 & $\begin{array}{l}\text { March } \\
\text { June } \\
\text { Sept } \\
\text { Dec }\end{array}$ & $\begin{array}{l}22.5 \\
23.1 \\
21.5 \\
22.0\end{array}$ & $\begin{array}{l}59.0 \\
59.5 \\
60.4 \\
60.2\end{array}$ & $\begin{array}{l}47.7 \\
49.0 \\
48.0 \\
49.1\end{array}$ \\
\hline 2007 & $\begin{array}{l}\text { Marchi, } \\
\text { June } e^{1, P} \\
\text { Sept } t^{1, P} \\
\text { Dec }^{1, P}\end{array}$ & $\begin{array}{l}22.7 \\
22.5 \\
21.1 \\
21.9\end{array}$ & $\begin{array}{l}62.5 \\
61.9 \\
57.9 \\
63.3\end{array}$ & $\begin{array}{l}50.7 \\
50.3 \\
48.6 \\
50.4\end{array}$ \\
\hline 200 & $\begin{array}{l}\text { March } \\
\text { June } \text { e }^{3, P}\end{array}$ & $\begin{array}{l}22.6 \\
22.5\end{array}$ & $\begin{array}{l}62.1 \\
61.4\end{array}$ & $\begin{array}{l}50.6 \\
49.2\end{array}$ \\
\hline
\end{tabular}

\begin{tabular}{rrr}
101.6 & 233.3 & 28 \\
94.9 & 179.8 & 252.6 \\
98.8 & 157.6 & 218.5 \\
97.7 & 159.0 & 209.7 \\
96.0 & 161.6 & 199.6 \\
97.1 & 167.8 & 199.4 \\
98.6 & 175.3 & 199.3 \\
101.3 & 181.3 & 205.5 \\
102.3 & 185.5 & 21 \\
103.1 & 191.2 & 222 \\
106.1 & 198.3 & 23 \\
25.1 & 45.4 & 5 \\
25.1 & 45.2 & 5 \\
25.3 & 45.6 & 5 \\
26.8 & 49.3 & 56.8 \\
25.4 & 47.5 & 5 \\
25.7 & 46.9 & 5 \\
24.7 & 46.3 & 55.8 \\
27.3 & 50.6 & 58.9 \\
26.7 & 49.4 & 57.0 \\
26.9 & 49.3 & 57.4 \\
25.6 & 48.5 & 58.8 \\
26.9 & 51.0 & 6 \\
26.8 & 49.7 & 59.1 \\
25.9 & 48.4 & 56.8 \\
\hline
\end{tabular}

$\begin{array}{rr}281.5 & 167 \\ 252.6 & 200.0 \\ 218.5 & 197.1 \\ 209.3 & 195.3 \\ 199.3 & 19 \\ 199.4 & 20 \\ 199.8 & 209 \\ 205.1 & 209 . \\ 211.3 & 209 . \\ 222.2 & 21 \\ 234.2 & 21 \\ 50.8 & 5 \\ 51.0 & 5 \\ 53.3 & 5 \\ 56.2 & 54 . \\ 54.2 & 5 \\ 53.8 & 5 \\ 55.3 & 53.4 \\ 58.9 & 55.1 \\ 57.0 & 5 \\ 57.4 & 5 \\ 58.8 & 5 \\ 61.1 & 53.6 \\ 59.1 & 5 \\ 56.8 & 49.1\end{array}$

$\begin{array}{rrr}167.5 & 57.6 & 12.1 \\ 200.0 & 75.5 & 14.1 \\ 197.1 & 86.0 & 16.0 \\ 195.3 & 88.7 & 17.0 \\ 196.7 & 92.2 & 17.8 \\ 204.3 & 98.9 & 19.6 \\ 209.0 & 103.1 & 20.9 \\ 209.6 & 106.8 & 22.8 \\ 209.2 & 110.0 & 23.6 \\ 212.4 & 115.4 & 25.5 \\ 211.1 & 117.8 & 26.5 \\ 51.0 & 26.6 & 5.7 \\ 50.7 & 26.9 & 5.8 \\ 53.1 & 27.5 & 6.0 \\ 54.3 & 29.1 & 6.0 \\ 52.4 & 28.3 & 6.2 \\ 51.4 & 28.3 & 6.5 \\ 53.6 & 28.9 & 6.4 \\ 55.1 & 29.9 & 6.5 \\ 52.7 & 29.3 & 6.5 \\ 52.0 & 29.0 & 6.5 \\ 52.9 & 29.7 & 6.7 \\ 53.6 & 29.8 & 6.8 \\ 52.3 & 29.1 & 6.6 \\ 49.1 & 27.9 & 6.5 \\ & & \end{array}$

103.9

$\begin{array}{ll}63.1 & 103.9 \\ 62.5 & 103 \\ 60.8 & 102 \\ 60.6 & 104\end{array}$

118.0

10.5
104.4
107.2

107.2
109.0
108.7

108.7
109.5
111.4

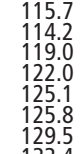

95.3
95.3

96.7
101.7

106.0
109.6
112.0

108.9
106.7
105.7
1136

$\begin{array}{ll}60.0 & 108.9 \\ 59.1 & 106.7 \\ 59.0 & 105.7 \\ 62.4 & 113.6 \\ 6.3 & \end{array}$

133.4

$\begin{array}{ll}60.3 & 111.2 \\ 60.3 & 108.1 \\ 57.2 & 104.9 \\ 63.0 & 114.1\end{array}$

123.8

125.9
131.7

117.5

109.8
108.5
113.3
116.7

129.2
126.1
127.5

116.2

$135.1-122.7$

$\begin{array}{ll}62.9 & 113.4 \\ 62.6 & 111.3 \\ 59.0 & 107.8\end{array}$

132.8

107.8

$\begin{array}{ll}62.9 & 110.4 \\ 60.9 & 106.8\end{array}$

13.6
132.2
136.2

121.2

123.1

132.5

122.1

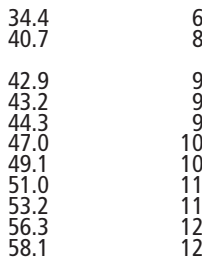

9.1
9.4
9.6
10.3
10.7
11.4
11.5
12.3
12.6

51.8
52.0
52.8

52.8
55.9

11.4
11.4
11.7

55.7
55.3
55.0

55.3
56.0
58.2

12.2
12.5
12.1
12.3

58.4
57.2

58.2
58.3
58.7

12.6
12.5
12.6
12.8

58.7
58.4

106.8

12.7

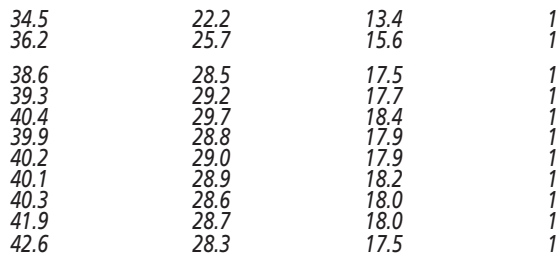

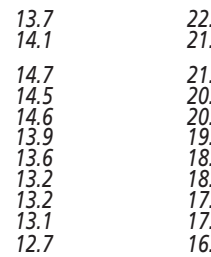

22.0

Note: Conception figures are estimates derived from birth registrations and abortion notifications. Rates for women of all ages, under 16, under 18 , under 20 and 40 and over are based on the population of women aged 15-44, 13-15, 15-17, 15-19 and 40-44 respectively. For a quarterly analysis of conceptions to women under 18 for local authority areas see the ONS website, www.ons.gov.uk

1 Figures for conceptions in 2007 exclude maternities relating to births in 2008 where the mother's date of birth was not stated on the registration and could not be supplied from another source. See Explanatory notes in the 2007 Conceptions report in Health Statistics Quarterly No. 41.

2 Rates for 2007 annual and quarterly conceptions and 2008 quarterly conceptions are calculated using 2007 mid-year population estimates.

3 Figures for conceptions by age for the March and June quarters of 2008 exclude maternities relating to births in 2008 where the mother's date of birth was not stated on the registration and could not be supplied from another source. Figures for conceptions by age for the March and June quarters of 2008 exclude maternities relating to births in 2009 where the mother's age was not recorded.

p provisional 


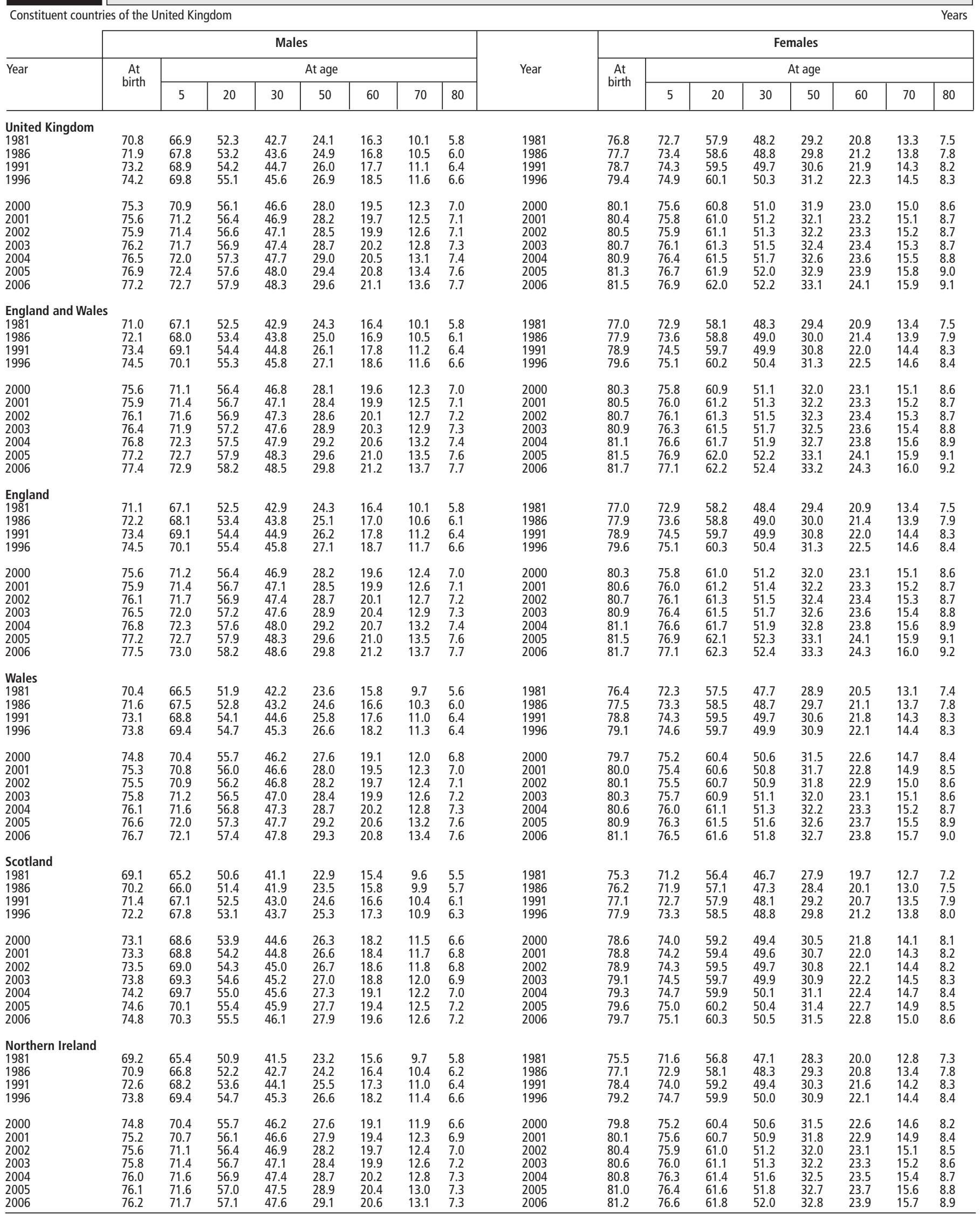

Note: All figures are based on a three-year period, so that for instance 2003 represents 2002-2004. The population estimates used to calculate these life expectancies are the latest available at time of publication of the 2005-2007 interim life tables (30 October 2008). All figures are based on death registrations. A minor methodological change was introduced for the 2006 figures to ensure consistency with population estimates of the very elderly for England and Wales. The effect on calculated life expectancies is marginal. 


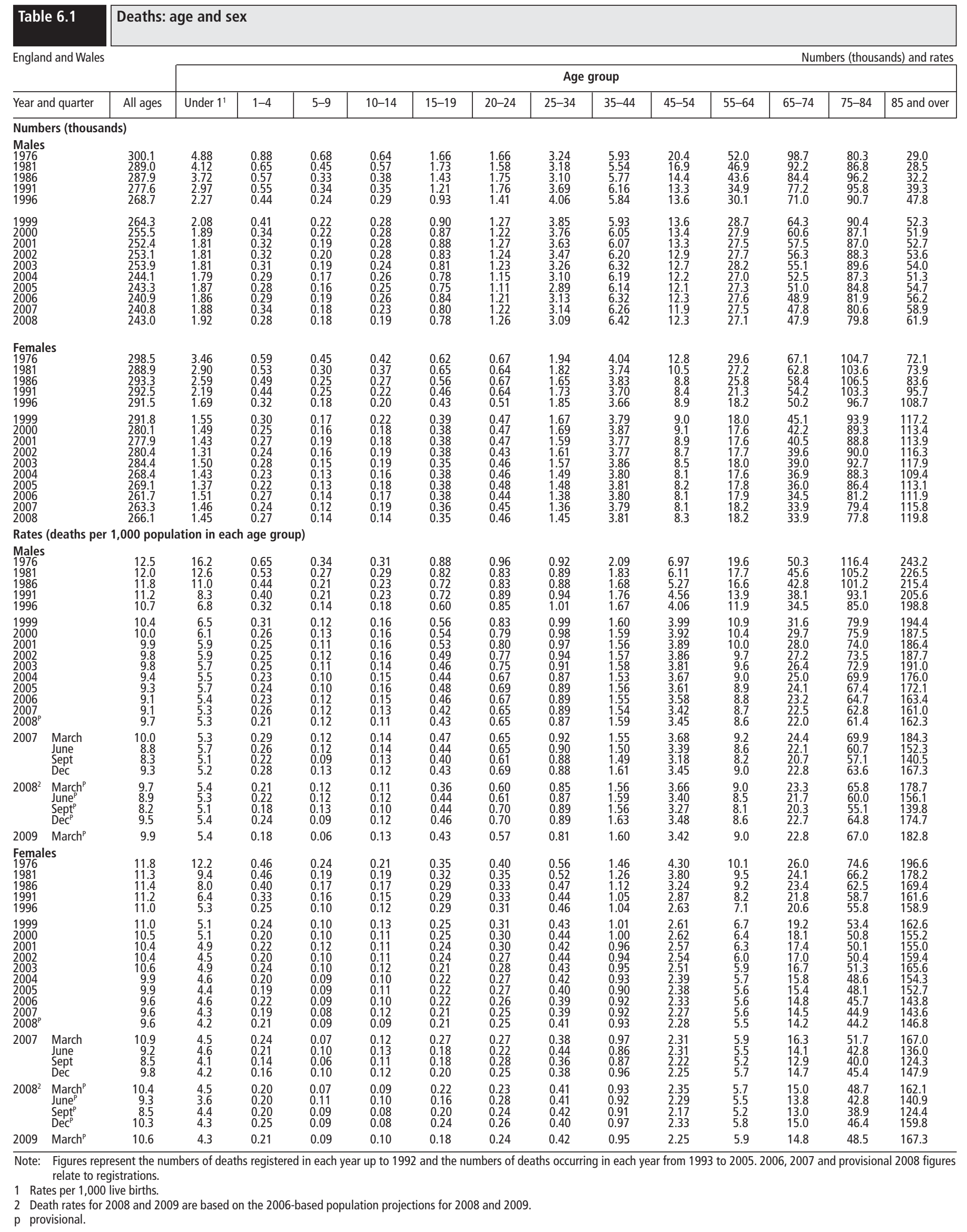




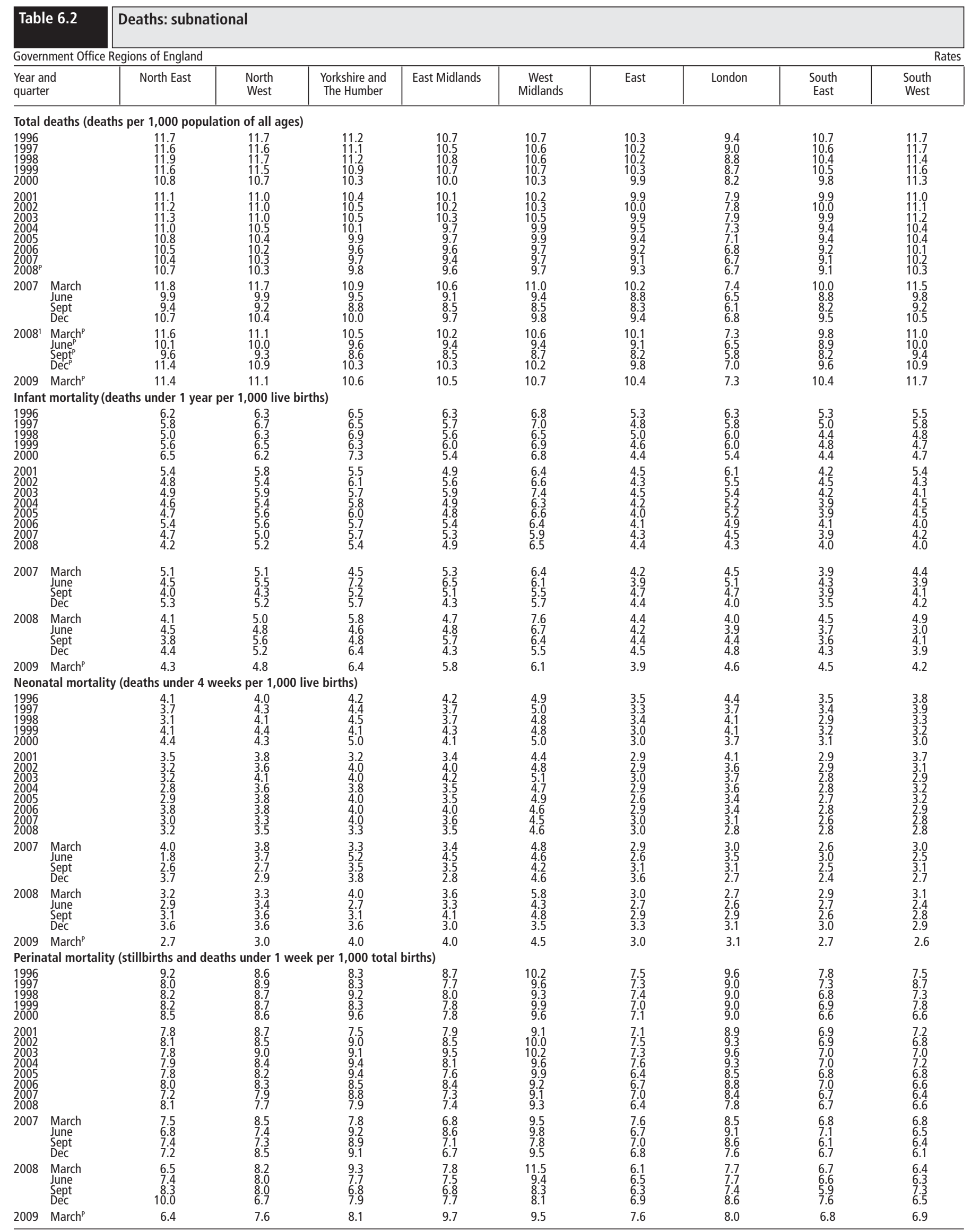

Note: Figures represent the numbers of deaths occurring in each year up to 2005. From 2006 onwards the figures relate to the numbers of deaths registered in each year. 1 Total deaths rates for 2008 and 2009 have been calculated using the mid-2007 population estimates published on 21 August 2008.
p provisional. 


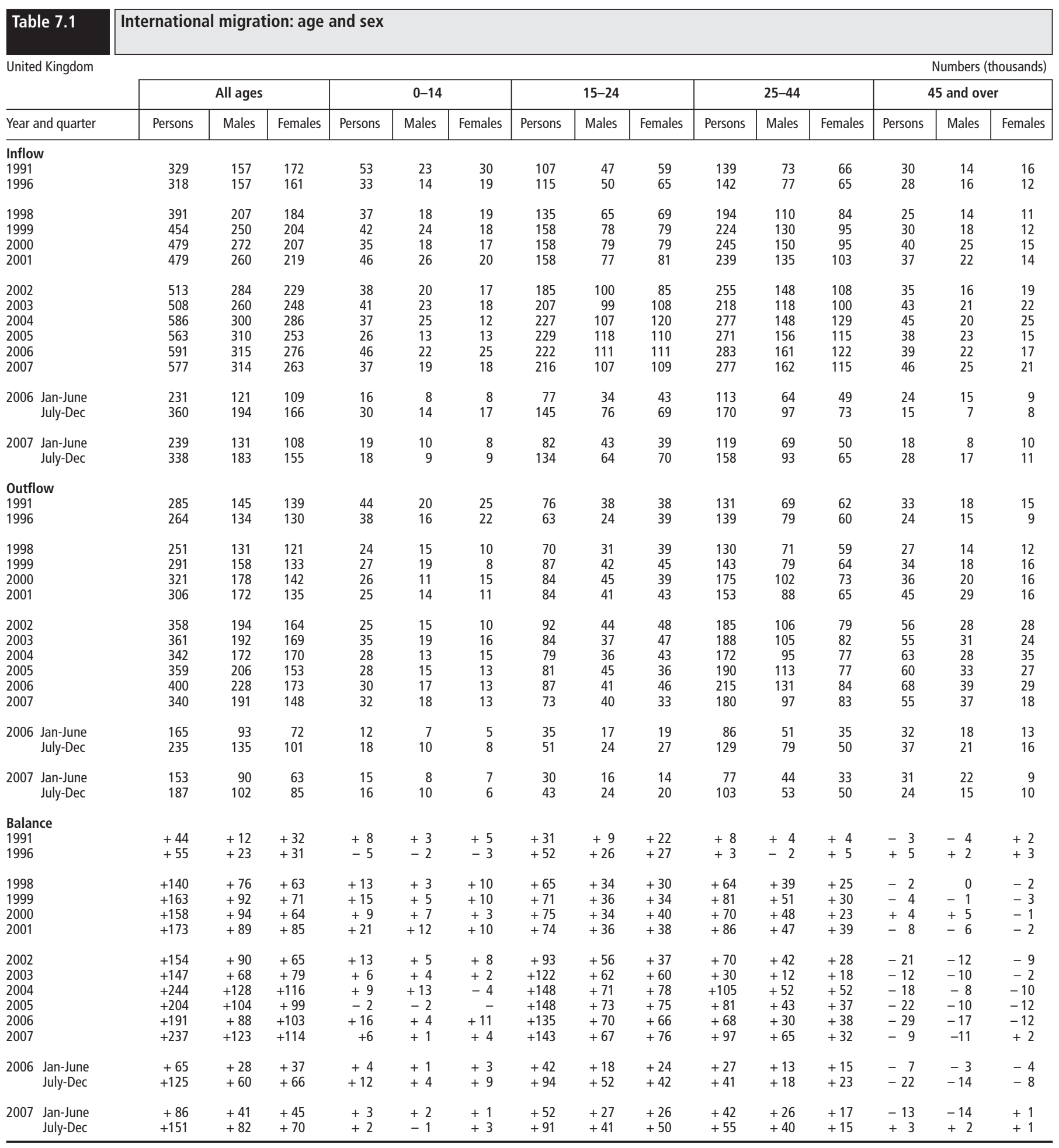

Note: These data have been revised following changes to the TIM methodology. Therefore they may not agree with estimates that have been published previously. 


\begin{tabular}{|c|c|c|c|c|c|c|c|c|c|c|c|}
\hline \multirow{2}{*}{$\begin{array}{l}\text { Table } 7.2 \\
\text { United Kingdom }\end{array}$} & \multicolumn{11}{|c|}{ International migration: country of last or next residence } \\
\hline & & & & & & & & & \multicolumn{3}{|c|}{ Numbers (thousands } \\
\hline & & & \multicolumn{6}{|c|}{ Commonwealth countries } & \multicolumn{3}{|c|}{ Other foreign countries } \\
\hline Year and quarter & $\begin{array}{c}\text { All } \\
\text { countries }\end{array}$ & $\begin{array}{c}\text { European } \\
\text { Union }\end{array}$ & $\begin{array}{l}\text { Australia, } \\
\text { New } \\
\text { Zealand, } \\
\text { Canada }\end{array}$ & $\begin{array}{l}\text { South } \\
\text { Africa }\end{array}$ & $\begin{array}{c}\text { India, } \\
\text { Bangladesh, } \\
\text { Sri Lanka }\end{array}$ & Pakistan & Caribbean & Other & USA & $\begin{array}{l}\text { Middle } \\
\text { East }\end{array}$ & Other \\
\hline $\begin{array}{l}\text { Inflow } \\
1991 \\
1996\end{array}$ & $\begin{array}{l}329 \\
318\end{array}$ & $\begin{array}{l}95 \\
98\end{array}$ & $\begin{array}{l}44 \\
37\end{array}$ & $\begin{array}{r}7 \\
11\end{array}$ & $\begin{array}{l}17 \\
15\end{array}$ & $\begin{array}{l}16 \\
11\end{array}$ & $\begin{array}{l}4 \\
4\end{array}$ & $\begin{array}{l}42 \\
33\end{array}$ & $\begin{array}{l}24 \\
32\end{array}$ & $\begin{array}{l}11 \\
14\end{array}$ & $\begin{array}{l}69 \\
63\end{array}$ \\
\hline $\begin{array}{l}1998 \\
1999 \\
2000 \\
2001\end{array}$ & $\begin{array}{l}391 \\
454 \\
479 \\
479\end{array}$ & $\begin{array}{r}109 \\
96 \\
89 \\
83\end{array}$ & $\begin{array}{l}64 \\
63 \\
63 \\
76\end{array}$ & $\begin{array}{l}20 \\
29 \\
22 \\
23\end{array}$ & $\begin{array}{l}18 \\
26 \\
34 \\
32\end{array}$ & $\begin{array}{l}10 \\
13 \\
16 \\
19\end{array}$ & $\begin{array}{l}6 \\
7 \\
6 \\
4\end{array}$ & $\begin{array}{l}31 \\
37 \\
48 \\
46\end{array}$ & $\begin{array}{l}37 \\
31 \\
24 \\
25\end{array}$ & $\begin{array}{l}13 \\
15 \\
30 \\
31\end{array}$ & $\begin{array}{r}84 \\
138 \\
146 \\
142\end{array}$ \\
\hline $\begin{array}{l}2002 \\
2003 \\
2004 \\
2005 \\
2006 \\
2007\end{array}$ & $\begin{array}{l}513 \\
508 \\
586 \\
563 \\
591 \\
577\end{array}$ & $\begin{array}{r}87 \\
98 \\
150 \\
182 \\
205 \\
222\end{array}$ & $\begin{array}{l}59 \\
64 \\
59 \\
61 \\
60 \\
47\end{array}$ & $\begin{array}{l}28 \\
28 \\
37 \\
29 \\
21 \\
17\end{array}$ & $\begin{array}{l}36 \\
45 \\
62 \\
62 \\
69 \\
65\end{array}$ & $\begin{array}{l}11 \\
13 \\
28 \\
24 \\
32 \\
30\end{array}$ & $\begin{array}{l}5 \\
4 \\
6 \\
2 \\
3 \\
3\end{array}$ & $\begin{array}{l}50 \\
51 \\
56 \\
41 \\
34 \\
37\end{array}$ & $\begin{array}{l}29 \\
30 \\
27 \\
25 \\
23 \\
23\end{array}$ & $\begin{array}{l}33 \\
26 \\
29 \\
19 \\
21 \\
23\end{array}$ & $\begin{array}{l}176 \\
150 \\
131 \\
118 \\
122 \\
109\end{array}$ \\
\hline $\begin{array}{l}2006 \begin{array}{l}\text { Jan-June } \\
\text { July-Dec }\end{array}\end{array}$ & $\begin{array}{l}231 \\
360\end{array}$ & $\begin{array}{r}72 \\
133\end{array}$ & $\begin{array}{l}28 \\
32\end{array}$ & $\begin{array}{r}13 \\
7\end{array}$ & $\begin{array}{l}26 \\
43\end{array}$ & $\begin{array}{l}13 \\
19\end{array}$ & $\begin{array}{l}1 \\
2\end{array}$ & $\begin{array}{l}14 \\
20\end{array}$ & $\begin{array}{r}9 \\
14\end{array}$ & $\begin{array}{r}8 \\
14\end{array}$ & $\begin{array}{l}46 \\
76\end{array}$ \\
\hline $\begin{array}{l}2007 \begin{array}{l}\text { Jan-June } \\
\text { June-Dec }\end{array}\end{array}$ & $\begin{array}{l}239 \\
338\end{array}$ & $\begin{array}{r}97 \\
125\end{array}$ & $\begin{array}{l}25 \\
22\end{array}$ & $\begin{array}{r}7 \\
10\end{array}$ & $\begin{array}{l}28 \\
37\end{array}$ & $\begin{array}{l}14 \\
17\end{array}$ & $\begin{array}{l}1 \\
2\end{array}$ & $\begin{array}{l}12 \\
25\end{array}$ & $\begin{array}{l}10 \\
12\end{array}$ & $\begin{array}{r}9 \\
15\end{array}$ & $\begin{array}{l}36 \\
72\end{array}$ \\
\hline $\begin{array}{l}\text { Outflow } \\
1991 \\
1996\end{array}$ & $\begin{array}{l}285 \\
264\end{array}$ & $\begin{array}{l}95 \\
94\end{array}$ & $\begin{array}{l}61 \\
58\end{array}$ & $\begin{array}{l}7 \\
5\end{array}$ & $\begin{array}{l}6 \\
5\end{array}$ & $\begin{array}{l}4 \\
1\end{array}$ & $\begin{array}{l}2 \\
1\end{array}$ & $\begin{array}{l}21 \\
23\end{array}$ & $\begin{array}{l}35 \\
26\end{array}$ & $\begin{array}{r}14 \\
8\end{array}$ & $\begin{array}{l}40 \\
42\end{array}$ \\
\hline $\begin{array}{l}1998 \\
1999 \\
2000 \\
2001\end{array}$ & $\begin{array}{l}251 \\
291 \\
321 \\
306\end{array}$ & $\begin{array}{r}85 \\
103 \\
103 \\
92\end{array}$ & $\begin{array}{l}54 \\
73 \\
79 \\
80\end{array}$ & $\begin{array}{l}6 \\
7 \\
7 \\
8\end{array}$ & $\begin{array}{l}5 \\
4 \\
5 \\
8\end{array}$ & $\begin{array}{l}2 \\
1 \\
3 \\
3\end{array}$ & $\begin{array}{l}2 \\
3 \\
3 \\
2\end{array}$ & $\begin{array}{l}14 \\
14 \\
15 \\
13\end{array}$ & $\begin{array}{l}27 \\
33 \\
33 \\
28\end{array}$ & $\begin{array}{r}9 \\
10 \\
15 \\
9\end{array}$ & $\begin{array}{l}48 \\
44 \\
58 \\
63\end{array}$ \\
\hline $\begin{array}{l}2002 \\
2003 \\
2004 \\
2005 \\
2006 \\
2007\end{array}$ & $\begin{array}{l}358 \\
361 \\
342 \\
359 \\
400 \\
340\end{array}$ & $\begin{array}{l}124 \\
121 \\
123 \\
136 \\
145 \\
130\end{array}$ & $\begin{array}{r}84 \\
90 \\
86 \\
86 \\
100 \\
83\end{array}$ & $\begin{array}{r}10 \\
14 \\
9 \\
13 \\
14 \\
11\end{array}$ & $\begin{array}{r}7 \\
7 \\
5 \\
9 \\
14 \\
14\end{array}$ & $\begin{array}{l}4 \\
4 \\
4 \\
7 \\
2 \\
5\end{array}$ & $\begin{array}{l}2 \\
1 \\
3 \\
2 \\
2 \\
1\end{array}$ & $\begin{array}{l}16 \\
15 \\
19 \\
11 \\
15 \\
13\end{array}$ & $\begin{array}{l}37 \\
27 \\
25 \\
24 \\
29 \\
18\end{array}$ & $\begin{array}{c}12 \\
7 \\
11 \\
11 \\
16 \\
11\end{array}$ & $\begin{array}{l}62 \\
75 \\
57 \\
60 \\
61 \\
53\end{array}$ \\
\hline $\begin{array}{l}2006 \begin{array}{l}\text { Jan-June } \\
\text { July-Dec }\end{array}\end{array}$ & $\begin{array}{l}165 \\
235\end{array}$ & $\begin{array}{l}60 \\
85\end{array}$ & $\begin{array}{l}44 \\
56\end{array}$ & $\begin{array}{l}6 \\
8\end{array}$ & $\begin{array}{l}5 \\
9\end{array}$ & $\begin{array}{l}1 \\
1\end{array}$ & $\begin{array}{l}1 \\
1\end{array}$ & $\begin{array}{r}5 \\
10\end{array}$ & $\begin{array}{l}11 \\
19\end{array}$ & $\begin{array}{r}6 \\
10\end{array}$ & $\begin{array}{l}27 \\
35\end{array}$ \\
\hline $\begin{array}{l}2007 \text { Jan-June } \\
\text { July-Dec }\end{array}$ & $\begin{array}{l}153 \\
187\end{array}$ & $\begin{array}{l}69 \\
62\end{array}$ & $\begin{array}{l}34 \\
49\end{array}$ & $\begin{array}{l}5 \\
6\end{array}$ & $\begin{array}{l}5 \\
8\end{array}$ & $\begin{array}{l}2 \\
3\end{array}$ & $\begin{array}{l}1 \\
1\end{array}$ & $\begin{array}{l}5 \\
8\end{array}$ & $\begin{array}{r}7 \\
12\end{array}$ & $\begin{array}{l}5 \\
6\end{array}$ & $\begin{array}{l}21 \\
32\end{array}$ \\
\hline $\begin{array}{l}\text { Balance } \\
1991 \\
1996\end{array}$ & $\begin{array}{l}+44 \\
+55\end{array}$ & $+\overline{5}$ & $\begin{array}{l}-18 \\
-21\end{array}$ & $\begin{array}{l}+1 \\
+5\end{array}$ & $\begin{array}{l}+11 \\
+10\end{array}$ & $\begin{array}{l}+12 \\
+10\end{array}$ & $\begin{array}{l}+2 \\
+3\end{array}$ & $\begin{array}{l}+21 \\
+10\end{array}$ & $\begin{array}{r}-10 \\
+7\end{array}$ & $\begin{array}{l}-3 \\
+5\end{array}$ & $\begin{array}{l}+29 \\
+21\end{array}$ \\
\hline $\begin{array}{l}1998 \\
1999 \\
2000 \\
2001\end{array}$ & $\begin{array}{l}+140 \\
+163 \\
+158 \\
+173\end{array}$ & $\begin{array}{r}+24 \\
-7 \\
-14 \\
-9\end{array}$ & $\begin{array}{r}+10 \\
-10 \\
-16 \\
-4\end{array}$ & $\begin{array}{l}+15 \\
+22 \\
+15 \\
+14\end{array}$ & $\begin{array}{l}+12 \\
+22 \\
+29 \\
+24\end{array}$ & $\begin{array}{r}+8 \\
+12 \\
+13 \\
+16\end{array}$ & $\begin{array}{l}+4 \\
+4 \\
+4 \\
+1\end{array}$ & $\begin{array}{l}+17 \\
+23 \\
+33 \\
+33\end{array}$ & $\begin{array}{r}+10 \\
-2 \\
-10 \\
-3\end{array}$ & $\begin{array}{r}+4 \\
+5 \\
+15 \\
+21\end{array}$ & $\begin{array}{l}+36 \\
+94 \\
+88 \\
+79\end{array}$ \\
\hline $\begin{array}{l}2002 \\
2003 \\
2004 \\
2005 \\
2006 \\
2007\end{array}$ & $\begin{array}{l}+154 \\
+147 \\
+244 \\
+204 \\
+191 \\
+237\end{array}$ & $\begin{array}{l}-37 \\
-23 \\
+27 \\
+46 \\
+60 \\
+91\end{array}$ & $\begin{array}{l}-25 \\
-26 \\
-26 \\
-25 \\
-41 \\
-36\end{array}$ & $\begin{array}{r}+17 \\
+14 \\
+28 \\
+15 \\
+7 \\
+6\end{array}$ & $\begin{array}{l}+29 \\
+38 \\
+56 \\
+53 \\
+55 \\
+51\end{array}$ & $\begin{array}{r}+7 \\
+9 \\
+25 \\
+18 \\
+30 \\
+26\end{array}$ & $\begin{array}{r}+3 \\
+3 \\
+3 \\
- \\
+1 \\
+2\end{array}$ & $\begin{array}{l}+34 \\
+36 \\
+38 \\
+30 \\
+19 \\
+24\end{array}$ & $\begin{array}{l}-9 \\
+3 \\
+2 \\
+1 \\
-6 \\
+4\end{array}$ & $\begin{array}{r}+21 \\
+19 \\
+18 \\
+8 \\
+5 \\
+12\end{array}$ & $\begin{array}{r}+114 \\
+75 \\
+74 \\
+58 \\
+61 \\
+55\end{array}$ \\
\hline $\begin{array}{l}2006 \text { Jan-June } \\
\text { July-Dec }\end{array}$ & $\begin{array}{r}+65 \\
+125\end{array}$ & $\begin{array}{l}+13 \\
+47\end{array}$ & $\begin{array}{l}-16 \\
-25\end{array}$ & $\begin{array}{l}+7 \\
-1\end{array}$ & $\begin{array}{l}+21 \\
+34\end{array}$ & $\begin{array}{l}+13 \\
+18\end{array}$ & + & $\begin{array}{r}+9 \\
+10\end{array}$ & $\begin{array}{l}-2 \\
-4\end{array}$ & $\begin{array}{l}+1 \\
+4\end{array}$ & $\begin{array}{l}+19 \\
+42\end{array}$ \\
\hline $\begin{array}{l}2007 \text { Jan-June } \\
\text { July-Dec }\end{array}$ & $\begin{array}{r}+86 \\
+151\end{array}$ & $\begin{array}{l}+28 \\
+63\end{array}$ & $\begin{array}{r}-9 \\
-27\end{array}$ & $\begin{array}{l}+2 \\
+4\end{array}$ & $\begin{array}{l}+23 \\
+29\end{array}$ & $\begin{array}{l}+12 \\
+14\end{array}$ & $\begin{array}{l}+1 \\
+1\end{array}$ & $\begin{array}{r}+7 \\
+17\end{array}$ & $\begin{array}{l}+4 \\
+1\end{array}$ & $\begin{array}{l}+4 \\
+8\end{array}$ & $\begin{array}{l}+15 \\
+41\end{array}$ \\
\hline
\end{tabular}

Note: These data have been revised following changes to the TIM methodology. Therefore they may not agree with estimates that have been published previously. 


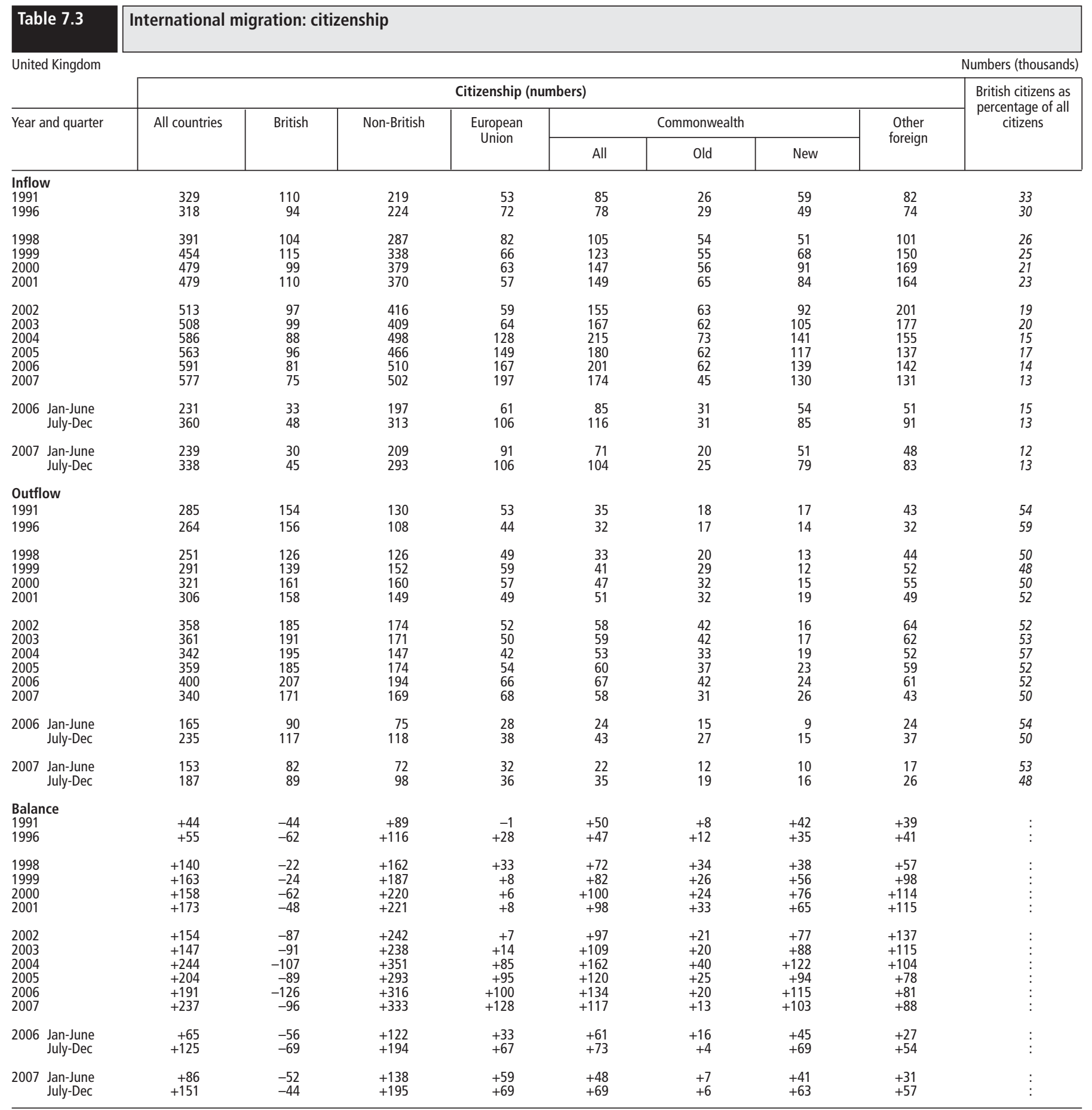

Note: These data have been revised following changes to the TIM methodology. Therefore they may not agree with estimates that have been published previously. 


\begin{tabular}{|c|c|c|c|c|c|c|c|c|c|c|c|c|c|}
\hline \multirow[b]{2}{*}{ Year and quarter } & \multirow[b]{2}{*}{ England } & \multirow[b]{2}{*}{ Wales } & \multirow[b]{2}{*}{ Scotland } & \multirow[b]{2}{*}{$\begin{array}{l}\text { Northern } \\
\text { Ireland }\end{array}$} & \multicolumn{9}{|c|}{ Government Office Regions of England } \\
\hline & & & & & North East & $\begin{array}{l}\text { North } \\
\text { West }\end{array}$ & $\begin{array}{l}\text { Yorkshire } \\
\text { and The } \\
\text { Humber }\end{array}$ & $\begin{array}{c}\text { East } \\
\text { Midlands }\end{array}$ & $\begin{array}{c}\text { West } \\
\text { Midlands }\end{array}$ & East & London & $\begin{array}{c}\text { South } \\
\text { East }\end{array}$ & $\begin{array}{l}\text { South } \\
\text { West }\end{array}$ \\
\hline
\end{tabular}

\begin{tabular}{|c|c|c|c|c|c|c|c|c|c|c|c|c|c|c|}
\hline $\begin{array}{l}\text { Inflov } \\
1976 \\
1981 \\
1986 \\
1991\end{array}$ & & $\begin{array}{r}105.4 \\
93.7 \\
115.6 \\
95.8\end{array}$ & $\begin{array}{l}52.0 \\
44.6 \\
55.2 \\
51.5\end{array}$ & $\begin{array}{l}50.4 \\
45.4 \\
43.9 \\
55.8\end{array}$ & $\begin{array}{r}9.7 \\
6.8 \\
8.8 \\
12.5\end{array}$ & $\begin{array}{l}39.2 \\
31.1 \\
36.5 \\
40.2\end{array}$ & $\begin{array}{l}93.0 \\
79.3 \\
90.0 \\
96.1\end{array}$ & $\begin{array}{l}78.2 \\
68.3 \\
78.6 \\
85.0\end{array}$ & $\begin{array}{r}84.0 \\
76.6 \\
101.9 \\
89.6\end{array}$ & $\begin{array}{l}75.7 \\
66.9 \\
87.1 \\
82.7\end{array}$ & $\begin{array}{l}146.3 \\
121.4 \\
144.6 \\
122.1\end{array}$ & $\begin{array}{l}155.0 \\
182.8 \\
148.8\end{array}$ & $\begin{array}{l}215.4 \\
201.8 \\
243.3 \\
197.6\end{array}$ & $\begin{array}{l}123.8 \\
108.3 \\
148.8 \\
120.7\end{array}$ \\
\hline $\begin{array}{l}1994 \\
1995 \\
1996 \\
1997 \\
1998\end{array}$ & & $\begin{array}{l}103.4 \\
108.1 \\
111.1 \\
110.9 \\
111.2\end{array}$ & $\begin{array}{l}52.0 \\
54.7 \\
55.3 \\
58.5 \\
56.3\end{array}$ & $\begin{array}{l}51.7 \\
48.5 \\
47.0 \\
55.3 \\
52.6\end{array}$ & $\begin{array}{l}10.9 \\
14.1 \\
11.4 \\
10.2 \\
11.7\end{array}$ & $\begin{array}{l}37.1 \\
37.9 \\
38.6 \\
38.6 \\
39.0\end{array}$ & $\begin{array}{r}99.7 \\
103.7 \\
105.0 \\
106.5 \\
104.0\end{array}$ & $\begin{array}{l}87.6 \\
90.8 \\
90.8 \\
92.6 \\
93.0\end{array}$ & $\begin{array}{r}96.4 \\
101.3 \\
102.1 \\
107.7 \\
107.9\end{array}$ & $\begin{array}{l}84.8 \\
90.0 \\
90.6 \\
92.7 \\
93.4\end{array}$ & $\begin{array}{l}130.6 \\
134.6 \\
139.5 \\
145.0 \\
142.8\end{array}$ & $\begin{array}{l}160.4 \\
170.7 \\
168.0 \\
167.3 \\
173.9\end{array}$ & $\begin{array}{l}215.5 \\
218.6 \\
228.0 \\
229.6 \\
226.1\end{array}$ & $\begin{array}{l}127.7 \\
131.6 \\
138.5 \\
144.0 \\
138.7\end{array}$ \\
\hline $\begin{array}{l}1999 \\
2000 \\
2001 \\
2002 \\
2003 \\
2004 \\
2005 \\
2006 \\
2007\end{array}$ & & $\begin{array}{r}111.7 \\
108.6 \\
104.2 \\
100.9 \\
97.5 \\
96.6 \\
98.3 \\
95.6 \\
92.0\end{array}$ & $\begin{array}{l}58.0 \\
59.5 \\
60.0 \\
64.0 \\
62.7 \\
60.1 \\
55.9 \\
56.5 \\
54.8\end{array}$ & $\begin{array}{l}50.9 \\
48.8 \\
56.5 \\
52.7 \\
59.8 \\
56.8 \\
59.2 \\
49.6 \\
55.6\end{array}$ & $\begin{array}{l}11.6 \\
11.2 \\
12.7 \\
10.8 \\
12.1 \\
12.5 \\
12.2 \\
13.0 \\
12.1\end{array}$ & $\begin{array}{l}38.7 \\
39.2 \\
40.4 \\
42.7 \\
41.9 \\
40.7 \\
39.9 \\
39.7 \\
38.8\end{array}$ & $\begin{array}{c}105.4 \\
106.2 \\
106.3 \\
108.9 \\
109.3 \\
104.9 \\
102.1 \\
100.1 \\
96.4\end{array}$ & $\begin{array}{l}95.2 \\
96.5 \\
96.5 \\
99.7 \\
99.4 \\
98.1 \\
94.1 \\
92.9 \\
91.2\end{array}$ & $\begin{array}{l}111.3 \\
112.1 \\
115.5 \\
119.5 \\
114.8 \\
111.8 \\
105.8 \\
106.9 \\
106.6\end{array}$ & $\begin{array}{l}93.7 \\
94.3 \\
95.3 \\
98.6 \\
95.0 \\
95.1 \\
94.0 \\
92.9 \\
91.3\end{array}$ & $\begin{array}{l}148.4 \\
145.8 \\
147.2 \\
150.0 \\
144.6 \\
145.5 \\
138.7 \\
143.9 \\
143.0\end{array}$ & $\begin{array}{l}162.9 \\
163.0 \\
159.7 \\
154.8 \\
148.3 \\
155.1 \\
161.2 \\
167.9 \\
163.6\end{array}$ & $\begin{array}{l}228.6 \\
224.2 \\
223.8 \\
228.6 \\
220.5 \\
223.4 \\
216.5 \\
224.7 \\
220.5\end{array}$ & $\begin{array}{l}143.2 \\
140.1 \\
143.3 \\
145.9 \\
141.6 \\
138.8 \\
132.3 \\
135.8 \\
134.4\end{array}$ \\
\hline 2007 & $\begin{array}{l}\text { March } \\
\text { June } \\
\text { Sept } \\
\text { Dec }\end{array}$ & $\begin{array}{l}19.2 \\
20.8 \\
31.5 \\
20.5\end{array}$ & $\begin{array}{l}11.0 \\
11.9 \\
20.5 \\
11.4\end{array}$ & $\begin{array}{l}13.6 \\
10.6 \\
21.1 \\
10.3\end{array}$ & $\begin{array}{l}3.6 \\
3.0 \\
2.7 \\
2.8\end{array}$ & $\begin{array}{r}7.2 \\
7.8 \\
15.7 \\
8.2\end{array}$ & $\begin{array}{l}19.9 \\
21.2 \\
34.7 \\
20.6\end{array}$ & $\begin{array}{l}17.3 \\
18.0 \\
37.9 \\
17.9\end{array}$ & $\begin{array}{l}21.1 \\
23.0 \\
39.9 \\
22.6\end{array}$ & $\begin{array}{l}19.1 \\
20.3 \\
31.4 \\
20.5\end{array}$ & $\begin{array}{l}30.3 \\
33.6 \\
47.3 \\
31.8\end{array}$ & $\begin{array}{l}36.6 \\
37.0 \\
51.9 \\
38.1\end{array}$ & $\begin{array}{l}46.2 \\
49.7 \\
75.9 \\
48.7\end{array}$ & $\begin{array}{l}27.0 \\
30.2 \\
47.9 \\
29.3\end{array}$ \\
\hline 2008 & $\begin{array}{l}\text { March } \\
\text { June } \\
\text { Sept }\end{array}$ & $\begin{array}{l}19.6 \\
20.9 \\
31.1\end{array}$ & $\begin{array}{l}10.5 \\
11.1 \\
18.7\end{array}$ & $\begin{array}{l}10.2 \\
11.8 \\
14.7\end{array}$ & $\begin{array}{l}3.8 \\
2.7 \\
2.4\end{array}$ & $\begin{array}{r}7.2 \\
7.6 \\
15.7\end{array}$ & $\begin{array}{l}19.7 \\
20.3 \\
34.7\end{array}$ & $\begin{array}{l}17.1 \\
17.7 \\
36.4\end{array}$ & $\begin{array}{l}20.2 \\
21.0 \\
38.1\end{array}$ & $\begin{array}{l}18.6 \\
19.9 \\
30.6\end{array}$ & $\begin{array}{l}29.1 \\
31.8 \\
42.1\end{array}$ & $\begin{array}{l}38.7 \\
39.5 \\
56.1\end{array}$ & $\begin{array}{l}43.8 \\
46.1 \\
69.6\end{array}$ & $\begin{array}{l}25.5 \\
27.4 \\
43.4\end{array}$ \\
\hline $\begin{array}{l}\text { Outfl } \\
1976 \\
1981 \\
1986 \\
1991\end{array}$ & & $\begin{array}{r}104.8 \\
91.5 \\
100.7 \\
112.2\end{array}$ & $\begin{array}{l}43.9 \\
41.8 \\
49.8 \\
47.4\end{array}$ & $\begin{array}{l}54.5 \\
47.7 \\
57.9 \\
46.7\end{array}$ & $\begin{array}{r}14.2 \\
9.4 \\
15.1 \\
9.3\end{array}$ & $\begin{array}{l}40.2 \\
39.1 \\
45.6 \\
40.9\end{array}$ & $\begin{array}{r}102.9 \\
98.6 \\
115.8 \\
104.9\end{array}$ & $\begin{array}{l}78.5 \\
73.3 \\
90.5 \\
85.4\end{array}$ & $\begin{array}{l}77.2 \\
71.7 \\
84.8 \\
81.4\end{array}$ & $\begin{array}{l}89.5 \\
78.4 \\
94.8 \\
87.9\end{array}$ & $\begin{array}{l}115.6 \\
104.4 \\
128.1 \\
113.0\end{array}$ & $\begin{array}{l}187.0 \\
232.4 \\
202.1\end{array}$ & $\begin{array}{l}181.7 \\
166.0 \\
204.1 \\
184.6\end{array}$ & $\begin{array}{r}94.7 \\
88.0 \\
102.5 \\
98.9\end{array}$ \\
\hline $\begin{array}{l}1994 \\
1995 \\
1996 \\
1997 \\
1998\end{array}$ & & $\begin{array}{l}106.3 \\
107.9 \\
105.3 \\
114.8 \\
111.3\end{array}$ & $\begin{array}{l}50.4 \\
53.1 \\
53.3 \\
54.4 \\
54.2\end{array}$ & $\begin{array}{l}49.0 \\
52.0 \\
54.5 \\
53.2 \\
53.8\end{array}$ & $\begin{array}{l}12.2 \\
12.3 \\
11.8 \\
12.6 \\
12.4\end{array}$ & $\begin{array}{l}43.5 \\
45.6 \\
44.5 \\
44.5 \\
43.7\end{array}$ & $\begin{array}{l}109.8 \\
115.8 \\
114.0 \\
117.5 \\
115.8\end{array}$ & $\begin{array}{r}91.9 \\
97.6 \\
98.2 \\
100.0 \\
97.9\end{array}$ & $\begin{array}{l}86.2 \\
91.9 \\
94.3 \\
97.4 \\
97.3\end{array}$ & $\begin{array}{r}95.1 \\
98.1 \\
101.0 \\
103.7 \\
100.9\end{array}$ & $\begin{array}{l}115.5 \\
118.7 \\
121.1 \\
124.8 \\
125.0\end{array}$ & $\begin{array}{l}206.3 \\
207.6 \\
213.4 \\
221.7 \\
217.9\end{array}$ & $\begin{array}{l}190.4 \\
195.8 \\
198.9 \\
205.7 \\
209.4\end{array}$ & $\begin{array}{l}103.9 \\
108.0 \\
109.8 \\
112.4 \\
110.9\end{array}$ \\
\hline $\begin{array}{l}1999 \\
2000 \\
2001 \\
2002 \\
2003 \\
2004 \\
2005 \\
2006 \\
2007\end{array}$ & & $\begin{array}{l}111.6 \\
110.8 \\
120.4 \\
119.3 \\
126.0 \\
121.5 \\
118.2 \\
110.4 \\
113.8\end{array}$ & $\begin{array}{l}53.3 \\
52.1 \\
51.5 \\
49.7 \\
48.1 \\
49.2 \\
50.0 \\
49.0 \\
48.4\end{array}$ & $\begin{array}{l}54.9 \\
53.3 \\
50.4 \\
48.4 \\
46.4 \\
45.1 \\
44.7 \\
44.1 \\
41.1\end{array}$ & $\begin{array}{l}12.5 \\
11.9 \\
11.1 \\
11.1 \\
11.7 \\
10.2 \\
12.7 \\
11.1 \\
11.2\end{array}$ & $\begin{array}{l}43.8 \\
42.9 \\
42.6 \\
41.3 \\
40.1 \\
39.4 \\
39.3 \\
39.1 \\
39.2\end{array}$ & $\begin{array}{l}114.9 \\
111.3 \\
110.4 \\
107.5 \\
104.1 \\
104.1 \\
103.1 \\
103.5 \\
103.6\end{array}$ & $\begin{array}{l}97.0 \\
95.7 \\
95.6 \\
94.6 \\
93.0 \\
92.2 \\
92.6 \\
94.2 \\
94.7\end{array}$ & $\begin{array}{l}96.4 \\
94.9 \\
95.6 \\
96.9 \\
96.0 \\
97.0 \\
96.7 \\
98.9 \\
97.8\end{array}$ & $\begin{array}{r}101.8 \\
101.5 \\
101.6 \\
102.7 \\
101.7 \\
100.7 \\
98.6 \\
100.9 \\
99.5\end{array}$ & $\begin{array}{l}125.8 \\
124.6 \\
127.1 \\
130.1 \\
127.4 \\
128.3 \\
123.7 \\
127.0 \\
123.6\end{array}$ & $\begin{array}{l}228.3 \\
231.5 \\
244.2 \\
262.5 \\
262.6 \\
260.2 \\
242.8 \\
246.7 \\
246.3\end{array}$ & $\begin{array}{l}208.7 \\
210.5 \\
216.4 \\
220.2 \\
211.1 \\
208.1 \\
201.0 \\
201.4 \\
198.1\end{array}$ & $\begin{array}{l}110.7 \\
110.7 \\
110.7 \\
111.0 \\
108.0 \\
108.4 \\
106.9 \\
107.9 \\
105.0\end{array}$ \\
\hline 2007 & $\begin{array}{l}\text { March } \\
\text { June } \\
\text { Sept } \\
\text { Dec }\end{array}$ & $\begin{array}{l}26.1 \\
23.7 \\
41.4 \\
22.5\end{array}$ & $\begin{array}{l}10.1 \\
11.1 \\
16.5 \\
10.7\end{array}$ & $\begin{array}{r}9.0 \\
9.6 \\
13.1 \\
9.3\end{array}$ & $\begin{array}{l}2.1 \\
2.0 \\
4.7 \\
2.3\end{array}$ & $\begin{array}{r}8.0 \\
8.9 \\
14.1 \\
8.2\end{array}$ & $\begin{array}{l}21.8 \\
22.9 \\
36.9 \\
22.0\end{array}$ & $\begin{array}{l}19.5 \\
21.9 \\
32.9 \\
20.4\end{array}$ & $\begin{array}{l}19.9 \\
22.5 \\
34.5 \\
20.9\end{array}$ & $\begin{array}{l}19.9 \\
22.2 \\
36.1 \\
21.3\end{array}$ & $\begin{array}{l}25.9 \\
25.7 \\
45.6 \\
26.4\end{array}$ & $\begin{array}{l}53.7 \\
54.1 \\
83.1 \\
55.3\end{array}$ & $\begin{array}{l}41.3 \\
43.1 \\
71.2 \\
42.5\end{array}$ & $\begin{array}{l}21.7 \\
22.4 \\
38.1 \\
22.7\end{array}$ \\
\hline 2008 & $\begin{array}{l}\text { March } \\
\text { June } \\
\text { Sept }\end{array}$ & $\begin{array}{l}22.6 \\
23.9 \\
33.6\end{array}$ & $\begin{array}{l}10.1 \\
11.1 \\
16.2\end{array}$ & $\begin{array}{r}9.6 \\
9.8 \\
13.1\end{array}$ & $\begin{array}{l}1.8 \\
1.7 \\
4.0\end{array}$ & $\begin{array}{r}7.9 \\
9.2 \\
14.2\end{array}$ & $\begin{array}{l}21.1 \\
23.0 \\
36.0\end{array}$ & $\begin{array}{l}19.0 \\
22.0 \\
32.3\end{array}$ & $\begin{array}{l}19.3 \\
21.7 \\
33.1\end{array}$ & $\begin{array}{l}20.0 \\
21.1 \\
35.0\end{array}$ & $\begin{array}{l}24.4 \\
24.4 \\
43.0\end{array}$ & $\begin{array}{l}50.8 \\
49.6 \\
72.7\end{array}$ & $\begin{array}{l}39.4 \\
41.2 \\
66.3\end{array}$ & $\begin{array}{l}21.0 \\
22.2 \\
36.7\end{array}$ \\
\hline $\begin{array}{l}\text { Balan } \\
1976 \\
1981 \\
1986 \\
1991\end{array}$ & & $\begin{array}{l}+0.6 \\
+2.1 \\
+14.9 \\
-16.4\end{array}$ & $\begin{array}{l}+8.1 \\
+2.7 \\
+5.4 \\
+4.0\end{array}$ & $\begin{array}{l}-4.1 \\
-2.3 \\
-14.1 \\
+\quad 9.2\end{array}$ & $\begin{array}{l}-4.5 \\
-2.5 \\
-6.3 \\
+3.2\end{array}$ & $\begin{array}{l}-1.0 \\
-8.0 \\
-9.1 \\
-0.7\end{array}$ & $\begin{array}{l}-9.8 \\
-19.3 \\
-25.8 \\
-8.8\end{array}$ & $\begin{array}{l}-0.3 \\
-5.0 \\
-11.9 \\
-0.4\end{array}$ & $\begin{array}{l}+6.8 \\
+4.9 \\
+17.1 \\
+8.1\end{array}$ & $\begin{array}{l}-13.8 \\
-11.6 \\
-7.8 \\
-5.2\end{array}$ & $\begin{array}{l}+30.7 \\
+17.0 \\
+16.5 \\
+9.1\end{array}$ & $\begin{array}{l}-32.0 \\
-49.6 \\
-53.3\end{array}$ & $\begin{array}{l}+33.7 \\
+35.8 \\
+39.2 \\
+13.0\end{array}$ & $\begin{array}{l}+29.1 \\
+20.3 \\
+46.4 \\
+21.8\end{array}$ \\
\hline $\begin{array}{l}1994 \\
1995 \\
1996 \\
1997 \\
1998\end{array}$ & & $\begin{array}{l}-2.9 \\
+0.2 \\
+5.8 \\
-3.8 \\
-0.1\end{array}$ & $\begin{array}{l}+1.5 \\
+1.6 \\
+2.0 \\
+4.1 \\
+2.1\end{array}$ & $\begin{array}{l}+2.6 \\
-3.5 \\
-7.5 \\
+2.2 \\
-1.2\end{array}$ & $\begin{array}{l}-1.2 \\
+1.8 \\
-0.4 \\
-2.4 \\
-0.8\end{array}$ & $\begin{array}{l}-6.4 \\
-7.7 \\
-5.9 \\
-5.9 \\
-4.8\end{array}$ & $\begin{array}{l}-10.1 \\
-12.1 \\
-9.0 \\
-11.0 \\
-11.8\end{array}$ & $\begin{array}{l}-4.4 \\
-6.8 \\
-7.4 \\
-7.3 \\
-4.9\end{array}$ & $\begin{array}{l}+10.2 \\
+9.4 \\
+7.8 \\
+10.3 \\
+10.6\end{array}$ & $\begin{array}{l}-10.3 \\
-8.1 \\
-10.4 \\
-11.1 \\
-7.4\end{array}$ & $\begin{array}{l}+15.1 \\
+15.9 \\
+18.3 \\
+20.3 \\
+17.7\end{array}$ & $\begin{array}{l}-45.9 \\
-36.9 \\
-45.4 \\
-54.5 \\
-44.0\end{array}$ & $\begin{array}{l}+25.1 \\
+22.7 \\
+29.1 \\
+23.8 \\
+16.7\end{array}$ & $\begin{array}{l}+23.8 \\
+23.6 \\
+28.7 \\
+31.6 \\
+27.8\end{array}$ \\
\hline $\begin{array}{l}1999 \\
2000 \\
2001 \\
2002 \\
2003 \\
2004 \\
2005 \\
2006 \\
2007\end{array}$ & & $\begin{array}{l}+0.1 \\
-2.2 \\
-16.3 \\
-18.4 \\
-28.5 \\
-25.0 \\
-19.9 \\
-14.9 \\
-21.8\end{array}$ & $\begin{array}{l}+4.7 \\
+7.4 \\
+8.5 \\
+14.3 \\
+14.6 \\
+10.9 \\
+5.9 \\
+7.4 \\
+6.4\end{array}$ & $\begin{array}{l}-4.0 \\
-4.5 \\
+6.1 \\
+4.3 \\
+13.4 \\
+11.7 \\
+14.5 \\
+5.5 \\
+14.5\end{array}$ & $\begin{array}{l}-0.8 \\
-0.7 \\
+1.6 \\
-0.3 \\
+0.4 \\
+2.3 \\
-0.5 \\
+2.0 \\
+0.9\end{array}$ & $\begin{array}{l}-5.1 \\
-3.7 \\
-2.3 \\
+1.4 \\
+1.8 \\
+1.3 \\
+0.6 \\
+0.6 \\
-0.4\end{array}$ & $\begin{array}{l}-9.5 \\
-5.1 \\
-4.1 \\
+1.4 \\
+5.2 \\
+0.8 \\
-1.0 \\
-3.5 \\
-7.2\end{array}$ & $\begin{array}{l}-1.8 \\
+0.8 \\
+0.9 \\
+5.0 \\
+6.4 \\
+5.9 \\
+1.5 \\
+1.3 \\
-3.5\end{array}$ & $\begin{array}{l}+14.9 \\
+17.2 \\
+19.9 \\
+22.6 \\
+18.7 \\
+14.8 \\
+9.2 \\
+8.1 \\
+8.8\end{array}$ & $\begin{array}{l}-8.1 \\
-7.2 \\
-6.3 \\
-4.1 \\
-6.7 \\
-5.6 \\
-4.6 \\
-8.0 \\
-8.2\end{array}$ & $\begin{array}{l}+22.6 \\
+21.2 \\
+20.1 \\
+19.9 \\
+17.2 \\
+17.2 \\
+15.1 \\
+16.9 \\
+19.4\end{array}$ & $\begin{array}{l}-65.4 \\
-68.6 \\
-84.5 \\
-107.8 \\
-114.3 \\
-105.1 \\
-81.5 \\
-78.8 \\
-82.6\end{array}$ & $\begin{array}{l}+19.8 \\
+13.8 \\
+7.4 \\
+8.4 \\
+9.4 \\
+15.3 \\
+15.5 \\
+23.3 \\
+22.4\end{array}$ & $\begin{array}{l}+32.6 \\
+29.3 \\
+32.6 \\
+34.8 \\
+33.6 \\
+30.5 \\
+25.4 \\
+27.9 \\
+29.4\end{array}$ \\
\hline 2007 & $\begin{array}{l}\text { March } \\
\text { June } \\
\text { Sept } \\
\text { Dec }\end{array}$ & $\begin{array}{l}-6.9 \\
-2.9 \\
-10.0 \\
-2.1\end{array}$ & $\begin{array}{l}+0.9 \\
+0.9 \\
+4.1 \\
+0.7\end{array}$ & $\begin{array}{l}+4.6 \\
+1.0 \\
+8.0 \\
+0.9\end{array}$ & $\begin{array}{l}+1.5 \\
+1.0 \\
-2.1 \\
+0.5\end{array}$ & $\begin{array}{l}-0.8 \\
-1.1 \\
+1.5 \\
-0.0\end{array}$ & $\begin{array}{l}-1.9 \\
-1.7 \\
-2.2 \\
-1.5\end{array}$ & $\begin{array}{l}-2.2 \\
-4.0 \\
+5.0 \\
-2.4\end{array}$ & $\begin{array}{l}+1.2 \\
+0.5 \\
+5.4 \\
+1.7\end{array}$ & $\begin{array}{l}-0.8 \\
-1.9 \\
-4.7 \\
-0.8\end{array}$ & $\begin{array}{l}+4.4 \\
+7.9 \\
+1.7 \\
+5.4\end{array}$ & $\begin{array}{l}-17.1 \\
-17.1 \\
-31.2 \\
-17.3\end{array}$ & $\begin{array}{l}+5.0 \\
+6.6 \\
+4.7 \\
+6.1\end{array}$ & $\begin{array}{l}+5.3 \\
+7.8 \\
+9.8 \\
+6.6\end{array}$ \\
\hline 2008 & $\begin{array}{l}\text { March } \\
\text { June } \\
\text { Sept }\end{array}$ & $\begin{array}{l}-3.0 \\
-3.1 \\
-2.5\end{array}$ & $\begin{array}{l}+0.4 \\
+0.0 \\
+2.5\end{array}$ & $\begin{array}{l}+0.6 \\
+2.0 \\
+1.6\end{array}$ & $\begin{array}{l}+2.0 \\
+1.0 \\
-1.6\end{array}$ & $\begin{array}{l}-0.8 \\
-1.6 \\
+1.5\end{array}$ & $\begin{array}{l}-1.5 \\
-2.7 \\
-1.4\end{array}$ & $\begin{array}{l}-1.8 \\
-4.3 \\
+4.2\end{array}$ & $\begin{array}{l}+0.9 \\
-0.7 \\
+5.1\end{array}$ & $\begin{array}{l}-1.4 \\
-1.2 \\
-4.4\end{array}$ & $\begin{array}{l}+4.6 \\
+7.5 \\
-1.0\end{array}$ & $\begin{array}{l}-12.1 \\
-10.0 \\
-16.6\end{array}$ & $\begin{array}{l}+4.4 \\
+4.9 \\
+3.3\end{array}$ & $\begin{array}{l}+4.5 \\
+5.2 \\
+6.7\end{array}$ \\
\hline
\end{tabular}

Note: Figures are derived from re-registrations recorded at the National Health Service Central Register. 


\begin{tabular}{|c|c|c|c|c|c|c|c|c|c|c|c|c|}
\hline \multirow{2}{*}{\multicolumn{2}{|c|}{$\begin{array}{l}\text { Table } 9.1 \\
\text { England and Wales }\end{array}$}} & \multicolumn{11}{|c|}{ First marriages ${ }^{1}$ : age and sex } \\
\hline & & \multicolumn{11}{|c|}{ Numbers (thousands), rates, percentages, mean and median age } \\
\hline & & \multicolumn{2}{|c|}{ All ages } & \multicolumn{6}{|c|}{ Persons marrying per 1,000 single population at ages } & \multirow{2}{*}{$\begin{array}{l}\text { Per cent } \\
\text { aged } \\
\text { under } 20\end{array}$} & \multirow{2}{*}{$\begin{array}{c}\text { Mean } \\
\text { age }^{3} \\
\text { (years) }\end{array}$} & \multirow{2}{*}{$\begin{array}{l}\text { Median } \\
\text { age }^{3} \\
\text { (years) }\end{array}$} \\
\hline \multicolumn{2}{|c|}{ Year and quarter } & Number & Rate $^{2}$ & $16-19$ & $20-24$ & $25-29$ & $30-34$ & $35-44$ & 45 and over & & & \\
\hline \multicolumn{2}{|c|}{$\begin{array}{l}\text { Males } \\
1961 \\
1966 \\
1971 \\
1976 \\
1981\end{array}$} & $\begin{array}{l}308.8 \\
339.1 \\
343.6 \\
274.4 \\
259.1\end{array}$ & $\begin{array}{l}74.9 \\
78.9 \\
82.3 \\
62.8 \\
51.7\end{array}$ & $\begin{array}{l}16.6 \\
22.1 \\
26.1 \\
18.5 \\
11.1\end{array}$ & $\begin{array}{r}159.1 \\
168.6 \\
167.7 \\
123.7 \\
94.1\end{array}$ & $\begin{array}{l}182.8 \\
185.4 \\
167.3 \\
132.5 \\
120.8\end{array}$ & $\begin{array}{l}91.9 \\
91.1 \\
84.6 \\
78.7 \\
70.3\end{array}$ & $\begin{array}{l}39.8 \\
36.4 \\
33.8 \\
32.0 \\
31.1\end{array}$ & $\begin{array}{l}9.3 \\
8.6 \\
8.0 \\
7.1 \\
5.4\end{array}$ & $\begin{array}{r}6.9 \\
9.9 \\
10.1 \\
9.8 \\
7.2\end{array}$ & $\begin{array}{l}25.6 \\
24.9 \\
24.6 \\
25.1 \\
25.4\end{array}$ & $\begin{array}{l}24.0 \\
23.4 \\
23.4 \\
23.7 \\
24.1\end{array}$ \\
\hline \multicolumn{2}{|l|}{$\begin{array}{l}1986 \\
1991\end{array}$} & $\begin{array}{l}253.0 \\
222.8\end{array}$ & $\begin{array}{l}45.0 \\
37.8\end{array}$ & $\begin{array}{l}6.0 \\
3.4\end{array}$ & $\begin{array}{l}64.4 \\
43.3\end{array}$ & $\begin{array}{r}105.1 \\
81.0\end{array}$ & $\begin{array}{l}73.9 \\
66.5\end{array}$ & $\begin{array}{l}30.9 \\
29.9\end{array}$ & $\begin{array}{l}4.8 \\
4.8\end{array}$ & $\begin{array}{l}3.8 \\
2.1\end{array}$ & $\begin{array}{l}26.3 \\
27.5\end{array}$ & $\begin{array}{l}25.1 \\
26.5\end{array}$ \\
\hline \multicolumn{2}{|c|}{$\begin{array}{l}1994 \\
1995 \\
1996 \\
1997 \\
1998\end{array}$} & $\begin{array}{l}206.1 \\
198.2 \\
193.3 \\
188.3 \\
186.3\end{array}$ & $\begin{array}{l}34.3 \\
32.4 \\
31.1 \\
29.7 \\
28.9\end{array}$ & $\begin{array}{l}2.2 \\
2.0 \\
1.8 \\
1.8 \\
1.7\end{array}$ & $\begin{array}{l}31.7 \\
28.3 \\
25.2 \\
22.8 \\
21.0\end{array}$ & $\begin{array}{l}73.3 \\
68.2 \\
64.5 \\
61.1 \\
59.4\end{array}$ & $\begin{array}{l}61.1 \\
59.9 \\
59.4 \\
58.0 \\
57.8\end{array}$ & $\begin{array}{l}30.2 \\
30.2 \\
30.7 \\
30.6 \\
30.2\end{array}$ & $\begin{array}{l}5.1 \\
5.0 \\
5.2 \\
5.2 \\
5.2\end{array}$ & $\begin{array}{l}1.3 \\
1.2 \\
1.1 \\
1.2 \\
1.2\end{array}$ & $\begin{array}{l}28.5 \\
28.9 \\
29.3 \\
29.6 \\
29.8\end{array}$ & $\begin{array}{l}27.5 \\
27.9 \\
28.3 \\
28.6 \\
28.9\end{array}$ \\
\hline \multicolumn{2}{|c|}{$\begin{array}{l}1999 \\
2000 \\
2001 \\
2002 \\
2003 \\
2004 \\
2005 \\
2006\end{array}$} & $\begin{array}{l}184.3 \\
186.1 \\
175.7 \\
179.1 \\
189.5 \\
192.0 \\
173.4 \\
169.2\end{array}$ & $\begin{array}{l}28.0 \\
27.7 \\
25.5 \\
25.4 \\
26.2 \\
25.9 \\
22.7 \\
21.6\end{array}$ & $\begin{array}{l}1.7 \\
1.7 \\
1.5 \\
1.3 \\
1.3 \\
1.2 \\
0.9 \\
0.8\end{array}$ & $\begin{array}{l}18.9 \\
18.2 \\
16.2 \\
16.2 \\
16.3 \\
15.7 \\
12.4 \\
11.1\end{array}$ & $\begin{array}{l}56.9 \\
54.3 \\
50.4 \\
48.7 \\
50.0 \\
49.0 \\
42.8 \\
40.3\end{array}$ & $\begin{array}{l}57.7 \\
58.2 \\
54.5 \\
55.7 \\
58.6 \\
58.8 \\
53.1 \\
51.2\end{array}$ & $\begin{array}{l}30.4 \\
32.0 \\
29.6 \\
31.0 \\
33.0 \\
33.7 \\
30.8 \\
29.9\end{array}$ & $\begin{array}{l}5.3 \\
5.7 \\
5.3 \\
5.9 \\
6.9 \\
7.2 \\
6.7 \\
6.9\end{array}$ & $\begin{array}{l}1.2 \\
1.2 \\
1.1 \\
1.0 \\
1.0 \\
1.0 \\
1.0 \\
0.6\end{array}$ & $\begin{array}{l}30.1 \\
30.5 \\
30.6 \\
30.9 \\
31.2 \\
31.4 \\
31.7 \\
31.8\end{array}$ & $\begin{array}{l}29.2 \\
29.6 \\
29.7 \\
30.1 \\
30.3 \\
30.4 \\
30.7 \\
30.8\end{array}$ \\
\hline \multicolumn{2}{|c|}{$2007^{\mathrm{P}}$} & 165.5 & 20.6 & 0.7 & 10.1 & 38.6 & 49.2 & 28.9 & 6.8 & 0.6 & 31.9 & 30.8 \\
\hline \multicolumn{2}{|c|}{$\begin{array}{c}2005 \text { March } \\
\text { June } \\
\text { Sept } \\
\text { Dec }\end{array}$} & $\begin{array}{l}20.0 \\
47.1 \\
77.3 \\
29.1\end{array}$ & $\begin{array}{l}10.6 \\
24.8 \\
40.2 \\
15.1\end{array}$ & $\begin{array}{l}0.8 \\
0.8 \\
1.2 \\
0.7\end{array}$ & $\begin{array}{r}7.1 \\
13.0 \\
21.3 \\
7.9\end{array}$ & $\begin{array}{l}18.5 \\
46.3 \\
79.9 \\
26.1\end{array}$ & $\begin{array}{l}22.7 \\
58.5 \\
95.3 \\
35.5\end{array}$ & $\begin{array}{l}14.9 \\
33.9 \\
51.6 \\
22.5\end{array}$ & $\begin{array}{l}3.7 \\
7.7 \\
9.8 \\
5.3\end{array}$ & $\begin{array}{l}1.5 \\
0.6 \\
0.6 \\
0.9\end{array}$ & $\begin{array}{l}31.6 \\
31.8 \\
31.4 \\
32.2\end{array}$ & $\begin{array}{l}30.6 \\
30.8 \\
30.5 \\
31.1\end{array}$ \\
\hline \multicolumn{2}{|c|}{$\begin{array}{l}2006 \text { March } \\
\text { June } \\
\text { Sept } \\
\text { Dec }\end{array}$} & $\begin{array}{l}16.7 \\
46.3 \\
78.4 \\
27.8\end{array}$ & $\begin{array}{r}8.7 \\
23.7 \\
39.7 \\
14.1\end{array}$ & $\begin{array}{l}0.5 \\
0.7 \\
1.1 \\
0.7\end{array}$ & $\begin{array}{r}5.4 \\
11.4 \\
20.0 \\
7.5\end{array}$ & $\begin{array}{l}14.5 \\
44.6 \\
77.5 \\
24.2\end{array}$ & $\begin{array}{l}18.9 \\
56.5 \\
95.6 \\
33.1\end{array}$ & $\begin{array}{l}12.7 \\
33.0 \\
52.7 \\
20.8\end{array}$ & $\begin{array}{r}3.7 \\
7.9 \\
10.6 \\
5.3\end{array}$ & $\begin{array}{l}1.1 \\
0.6 \\
0.5 \\
0.9\end{array}$ & $\begin{array}{l}32.1 \\
32.0 \\
31.6 \\
32.2\end{array}$ & $\begin{array}{l}30.9 \\
30.8 \\
30.6 \\
31.1\end{array}$ \\
\hline 2007 & $\begin{array}{l}\text { March }{ }^{p} \\
\text { June }{ }^{p} \\
\text { Septp }^{p} \\
\text { Dec }^{p}\end{array}$ & $\begin{array}{l}17.6 \\
46.4 \\
74.0 \\
27.4\end{array}$ & $\begin{array}{r}8.9 \\
23.1 \\
36.5 \\
13.5\end{array}$ & $\begin{array}{l}0.6 \\
0.7 \\
0.9 \\
0.6\end{array}$ & $\begin{array}{r}5.2 \\
10.9 \\
17.5 \\
6.8\end{array}$ & $\begin{array}{l}15.3 \\
43.4 \\
71.6 \\
23.5\end{array}$ & $\begin{array}{l}19.7 \\
56.4 \\
88.1 \\
32.1\end{array}$ & $\begin{array}{l}13.1 \\
32.4 \\
49.6 \\
20.2\end{array}$ & $\begin{array}{r}3.6 \\
7.7 \\
10.3 \\
5.3\end{array}$ & $\begin{array}{l}1.2 \\
0.6 \\
0.4 \\
0.8\end{array}$ & $\begin{array}{l}32.2 \\
32.0 \\
31.7 \\
32.3\end{array}$ & $\begin{array}{l}30.9 \\
30.8 \\
30.6 \\
31.1\end{array}$ \\
\hline $\begin{array}{l}\text { Fema } \\
1961 \\
1966 \\
1971 \\
1976 \\
1981\end{array}$ & & $\begin{array}{l}312.3 \\
342.7 \\
347.4 \\
276.5 \\
263.4\end{array}$ & $\begin{array}{l}83.0 \\
89.3 \\
97.0 \\
76.9 \\
64.0\end{array}$ & $\begin{array}{l}77.0 \\
82.6 \\
92.9 \\
66.7 \\
41.5\end{array}$ & $\begin{array}{l}261.1 \\
263.7 \\
246.5 \\
185.4 \\
140.8\end{array}$ & $\begin{array}{l}162.8 \\
153.4 \\
167.0 \\
140.7 \\
120.2\end{array}$ & $\begin{array}{l}74.6 \\
74.1 \\
75.7 \\
77.6 \\
67.0\end{array}$ & $\begin{array}{l}29.8 \\
30.2 \\
30.3 \\
31.6 \\
28.7\end{array}$ & $\begin{array}{l}4.6 \\
4.3 \\
4.8 \\
4.0 \\
2.8\end{array}$ & $\begin{array}{l}28.7 \\
32.5 \\
31.1 \\
31.1 \\
24.1\end{array}$ & $\begin{array}{l}23.1 \\
22.5 \\
22.6 \\
22.8 \\
23.1\end{array}$ & $\begin{array}{l}21.6 \\
21.2 \\
21.4 \\
21.5 \\
21.9\end{array}$ \\
\hline \multicolumn{2}{|c|}{$\begin{array}{l}1986 \\
1991\end{array}$} & $\begin{array}{l}256.8 \\
224.8\end{array}$ & $\begin{array}{l}55.6 \\
46.7\end{array}$ & $\begin{array}{l}24.1 \\
14.0\end{array}$ & $\begin{array}{r}102.4 \\
73.0\end{array}$ & $\begin{array}{r}108.7 \\
90.6\end{array}$ & $\begin{array}{l}67.1 \\
62.7\end{array}$ & $\begin{array}{l}28.6 \\
28.1\end{array}$ & $\begin{array}{l}2.7 \\
2.8\end{array}$ & $\begin{array}{r}13.9 \\
7.9\end{array}$ & $\begin{array}{l}24.1 \\
25.5\end{array}$ & $\begin{array}{l}23.1 \\
24.6\end{array}$ \\
\hline \multicolumn{2}{|c|}{$\begin{array}{l}1994 \\
1995 \\
1996 \\
1997 \\
1998\end{array}$} & $\begin{array}{l}206.3 \\
198.6 \\
192.7 \\
188.5 \\
187.4\end{array}$ & $\begin{array}{l}41.6 \\
39.3 \\
37.3 \\
35.6 \\
34.7\end{array}$ & $\begin{array}{l}9.6 \\
9.0 \\
8.0 \\
7.4 \\
7.2\end{array}$ & $\begin{array}{l}56.4 \\
50.8 \\
45.7 \\
42.5 \\
39.9\end{array}$ & $\begin{array}{l}84.5 \\
80.5 \\
77.2 \\
74.1 \\
72.6\end{array}$ & $\begin{array}{l}58.9 \\
57.1 \\
57.2 \\
56.1 \\
56.1\end{array}$ & $\begin{array}{l}27.7 \\
27.6 \\
27.8 \\
27.2 \\
26.5\end{array}$ & $\begin{array}{l}3.1 \\
3.1 \\
3.2 \\
3.3 \\
3.4\end{array}$ & $\begin{array}{l}5.2 \\
5.1 \\
4.9 \\
4.7 \\
4.7\end{array}$ & $\begin{array}{l}26.5 \\
26.8 \\
27.2 \\
27.5 \\
27.7\end{array}$ & $\begin{array}{l}25.7 \\
26.0 \\
26.4 \\
267 \\
27.0\end{array}$ \\
\hline \multicolumn{2}{|c|}{$\begin{array}{l}1999 \\
2000 \\
2001 \\
2002 \\
2003 \\
2004 \\
2005 \\
2006\end{array}$} & $\begin{array}{l}185.3 \\
187.7 \\
177.5 \\
180.7 \\
191.2 \\
194.3 \\
176.5 \\
172.8\end{array}$ & $\begin{array}{l}33.5 \\
33.2 \\
30.6 \\
30.4 \\
31.3 \\
31.0 \\
27.2 \\
25.9\end{array}$ & $\begin{array}{l}6.7 \\
6.5 \\
5.5 \\
5.4 \\
5.4 \\
4.9 \\
3.2 \\
2.8\end{array}$ & $\begin{array}{l}36.7 \\
35.2 \\
31.9 \\
30.9 \\
31.4 \\
30.2 \\
24.3 \\
22.0\end{array}$ & $\begin{array}{l}70.8 \\
68.7 \\
64.3 \\
63.2 \\
64.5 \\
63.6 \\
57.2 \\
54.3\end{array}$ & $\begin{array}{l}56.0 \\
57.2 \\
53.2 \\
54.5 \\
57.8 \\
58.9 \\
54.4 \\
52.4\end{array}$ & $\begin{array}{l}26.5 \\
27.5 \\
25.5 \\
26.8 \\
28.5 \\
29.0 \\
26.5 \\
25.5\end{array}$ & $\begin{array}{l}3.5 \\
3.9 \\
3.7 \\
4.3 \\
5.2 \\
5.6 \\
5.2 \\
5.7\end{array}$ & $\begin{array}{l}4.4 \\
4.2 \\
3.9 \\
3.7 \\
3.6 \\
3.4 \\
2.5 \\
2.2\end{array}$ & $\begin{array}{l}28.0 \\
28.2 \\
28.4 \\
28.7 \\
28.9 \\
29.1 \\
29.5 \\
29.7\end{array}$ & $\begin{array}{l}27.3 \\
27.5 \\
27.7 \\
27.9 \\
28.1 \\
28.3 \\
28.5 \\
28.6\end{array}$ \\
\hline \multicolumn{2}{|c|}{$2007^{p}$} & 168.3 & 24.5 & 2.5 & 20.3 & 52.0 & 50.5 & 24.1 & 5.5 & 2.1 & 29.8 & 28.7 \\
\hline 2005 & $\begin{array}{l}\text { March } \\
\text { June } \\
\text { Sept } \\
\text { Dec }\end{array}$ & $\begin{array}{l}20.0 \\
48.4 \\
78.9 \\
29.3\end{array}$ & $\begin{array}{l}12.5 \\
29.9 \\
48.3 \\
17.9\end{array}$ & $\begin{array}{l}3.0 \\
3.1 \\
4.2 \\
2.5\end{array}$ & $\begin{array}{l}12.0 \\
26.3 \\
44.2 \\
14.4\end{array}$ & $\begin{array}{r}22.7 \\
62.7 \\
107.7 \\
34.9\end{array}$ & $\begin{array}{l}23.9 \\
60.3 \\
94.5 \\
38.0\end{array}$ & $\begin{array}{l}12.9 \\
29.5 \\
42.8 \\
20.3\end{array}$ & $\begin{array}{l}3.2 \\
6.3 \\
7.1 \\
4.2\end{array}$ & $\begin{array}{l}5.1 \\
2.1 \\
1.8 \\
2.9\end{array}$ & $\begin{array}{l}29.4 \\
29.6 \\
29.2 \\
30.0\end{array}$ & $\begin{array}{l}28.4 \\
28.6 \\
28.3 \\
29.1\end{array}$ \\
\hline 2006 & $\begin{array}{l}\text { March } \\
\text { June } \\
\text { Sept } \\
\text { Dec }\end{array}$ & $\begin{array}{l}16.9 \\
47.4 \\
80.2 \\
28.3\end{array}$ & $\begin{array}{l}10.3 \\
28.5 \\
47.6 \\
16.8\end{array}$ & $\begin{array}{l}2.0 \\
2.6 \\
4.2 \\
2.4\end{array}$ & $\begin{array}{r}9.5 \\
23.7 \\
41.1 \\
13.6\end{array}$ & $\begin{array}{r}18.3 \\
59.2 \\
106.5 \\
32.4\end{array}$ & $\begin{array}{l}19.9 \\
59.3 \\
94.4 \\
35.5\end{array}$ & $\begin{array}{l}11.6 \\
28.5 \\
43.2 \\
18.5\end{array}$ & $\begin{array}{l}3.1 \\
6.9 \\
8.1 \\
4.6\end{array}$ & $\begin{array}{l}4.0 \\
1.9 \\
1.8 \\
2.9\end{array}$ & $\begin{array}{l}29.9 \\
29.9 \\
29.4 \\
30.1\end{array}$ & $\begin{array}{l}28.7 \\
28.7 \\
28.4 \\
29.1\end{array}$ \\
\hline 2007 & $\begin{array}{l}\text { March } \\
\text { June }{ }^{p} \\
\text { Sept } \\
\operatorname{Dec}^{p}\end{array}$ & $\begin{array}{l}18.0 \\
47.3 \\
75.5 \\
27.6\end{array}$ & $\begin{array}{l}10.6 \\
27.6 \\
43.6 \\
15.9\end{array}$ & $\begin{array}{l}2.0 \\
2.5 \\
3.3 \\
2.2\end{array}$ & $\begin{array}{r}9.2 \\
22.2 \\
36.9 \\
12.5\end{array}$ & $\begin{array}{l}19.8 \\
58.8 \\
97.7 \\
31.2\end{array}$ & $\begin{array}{l}21.4 \\
58.0 \\
88.1 \\
34.1\end{array}$ & $\begin{array}{l}11.6 \\
27.0 \\
39.8 \\
17.6\end{array}$ & $\begin{array}{l}3.0 \\
6.6 \\
7.9 \\
4.3\end{array}$ & $\begin{array}{l}3.9 \\
1.8 \\
1.5 \\
2.7\end{array}$ & $\begin{array}{l}29.9 \\
29.9 \\
29.5 \\
30.2\end{array}$ & $\begin{array}{l}28.8 \\
28.8 \\
28.5 \\
29.1\end{array}$ \\
\hline
\end{tabular}

Note: Rates have been revised from 2002, to include the adjustments for marriages to England and Wales residents occuring abroad - see 'In Brief'.

1 Figures for all marriages can be found in Table 2.1.

2 Per 1,000 single persons aged 16 and over.

3 The mean/median ages shown in this table are unstandardised and therefore take no account of changes in the structure of the population by age or marital status.

p provisional 


\begin{tabular}{|c|c|c|c|c|c|c|c|c|c|c|c|c|c|}
\hline \multirow{2}{*}{\multicolumn{2}{|c|}{$\begin{array}{l}\text { Table } 9.2 \\
\text { England and Wales }\end{array}$}} & \multicolumn{12}{|c|}{ Remarriages': age, sex, and previous marital status } \\
\hline & & \multicolumn{12}{|c|}{ Numbers (thousands), rates, percentages, mean and median age } \\
\hline & & \multicolumn{10}{|c|}{ Remarriages of divorced persons } & \multirow{2}{*}{\multicolumn{2}{|c|}{$\begin{array}{c}\text { Remarriages of widowed } \\
\text { persons }\end{array}$}} \\
\hline \multicolumn{2}{|c|}{ Year and quarter } & \multicolumn{2}{|c|}{ All ages } & \multicolumn{5}{|c|}{ Persons remarrying per 1,000 divorced population at ages } & \multirow{2}{*}{$\begin{array}{c}\text { Per cent } \\
\text { aged } \\
\text { under } 35\end{array}$} & \multirow{2}{*}{$\begin{array}{c}\text { Mean } \\
\text { age }^{3} \\
\text { (years) }\end{array}$} & \multirow{2}{*}{$\begin{array}{c}\text { Median } \\
\text { age }^{3} \\
\text { (years) }\end{array}$} & & \\
\hline & & Number & Rate $^{2}$ & $16-24$ & $25-29$ & $30-34$ & $35-44$ & 45 and over & & & & Number & Rate $^{4}$ \\
\hline $\begin{array}{l}\text { Males } \\
1961 \\
1966 \\
1971 \\
1976 \\
1981\end{array}$ & & $\begin{array}{l}18.8 \\
26.7 \\
42.4 \\
67.2 \\
79.1\end{array}$ & $\begin{array}{l}162.9 \\
192.2 \\
227.3 \\
178.8 \\
129.5\end{array}$ & $\begin{array}{l}478.6 \\
737.8 \\
525.2 \\
656.8 \\
240.7\end{array}$ & $\begin{array}{l}473.6 \\
522.5 \\
509.0 \\
359.7 \\
260.9\end{array}$ & $\begin{array}{l}351.6 \\
403.1 \\
390.7 \\
266.8 \\
205.8\end{array}$ & $\begin{array}{l}198.3 \\
244.4 \\
251.3 \\
187.9 \\
141.9\end{array}$ & $\begin{array}{r}88.6 \\
89.4 \\
124.8 \\
94.0 \\
63.9\end{array}$ & $\begin{array}{l}33.9 \\
40.8 \\
42.8 \\
46.7 \\
46.1\end{array}$ & $\begin{array}{l}40.5 \\
39.3 \\
39.8 \\
338.4 \\
38.1\end{array}$ & $\begin{array}{l}39.2 \\
37.4 \\
37.0 \\
36.0 \\
35.9\end{array}$ & $\begin{array}{l}19.1 \\
18.7 \\
18.7 \\
16.9 \\
13.8\end{array}$ & $\begin{array}{l}28.8 \\
28.3 \\
27.5 \\
24.7 \\
19.7\end{array}$ \\
\hline $\begin{array}{l}1986 \\
1991\end{array}$ & & $\begin{array}{l}83.4 \\
74.9\end{array}$ & $\begin{array}{l}91.0 \\
63.0\end{array}$ & $\begin{array}{r}141.4 \\
81.1\end{array}$ & $\begin{array}{l}158.9 \\
111.3\end{array}$ & $\begin{array}{l}141.3 \\
100.6\end{array}$ & $\begin{array}{r}106.0 \\
72.7\end{array}$ & $\begin{array}{l}49.9 \\
38.4\end{array}$ & $\begin{array}{l}38.5 \\
34.3\end{array}$ & $\begin{array}{l}39.1 \\
40.3\end{array}$ & $\begin{array}{l}37.7 \\
39.0\end{array}$ & $\begin{array}{r}11.6 \\
9.0\end{array}$ & $\begin{array}{l}16.7 \\
12.5\end{array}$ \\
\hline $\begin{array}{l}1994 \\
1995 \\
1996 \\
1997 \\
1998\end{array}$ & & $\begin{array}{l}76.6 \\
77.0 \\
78.0 \\
76.8 \\
74.0\end{array}$ & $\begin{array}{l}60.0 \\
58.6 \\
57.9 \\
55.7 \\
52.7\end{array}$ & $\begin{array}{l}180.6 \\
190.0 \\
166.2 \\
170.9 \\
167.0\end{array}$ & $\begin{array}{l}131.7 \\
132.1 \\
135.2 \\
132.2 \\
124.7\end{array}$ & $\begin{array}{l}110.2 \\
111.4 \\
111.2 \\
110.3 \\
104.1\end{array}$ & $\begin{array}{l}71.5 \\
72.2 \\
73.8 \\
72.9 \\
71.6\end{array}$ & $\begin{array}{l}36.1 \\
34.9 \\
35.0 \\
33.6 \\
32.0\end{array}$ & $\begin{array}{l}31.5 \\
30.3 \\
28.2 \\
27.0 \\
24.8\end{array}$ & $\begin{array}{l}41.1 \\
41.3 \\
41.7 \\
42.0 \\
42.4\end{array}$ & $\begin{array}{l}39.6 \\
39.8 \\
40.2 \\
40.5 \\
40.8\end{array}$ & $\begin{array}{l}8.4 \\
7.8 \\
7.7 \\
7.4 \\
6.9\end{array}$ & $\begin{array}{r}11.5 \\
10.8 \\
10.6 \\
10.3 \\
9.6\end{array}$ \\
\hline $\begin{array}{l}1999 \\
2000 \\
2001 \\
2002 \\
2003 \\
2004 \\
2005 \\
2006\end{array}$ & & $\begin{array}{l}72.6 \\
75.4 \\
67.7 \\
70.5 \\
74.4 \\
75.1 \\
68.7 \\
64.8\end{array}$ & $\begin{array}{l}50.7 \\
51.8 \\
45.7 \\
46.3 \\
47.4 \\
46.5 \\
41.3 \\
38.2\end{array}$ & $\begin{array}{r}125.7 \\
97.9 \\
75.7 \\
69.8 \\
77.7 \\
70.4 \\
34.9 \\
34.8\end{array}$ & $\begin{array}{c}120.7 \\
113.2 \\
96.6 \\
92.4 \\
92.0 \\
89.2 \\
67.5 \\
58.3\end{array}$ & $\begin{array}{r}102.9 \\
103.6 \\
95.8 \\
96.0 \\
94.7 \\
91.0 \\
79.8 \\
71.5\end{array}$ & $\begin{array}{l}70.2 \\
74.4 \\
67.6 \\
68.7 \\
70.6 \\
71.2 \\
63.7 \\
59.6\end{array}$ & $\begin{array}{l}31.2 \\
32.6 \\
28.5 \\
30.0 \\
31.8 \\
31.4 \\
22.0 \\
27.5\end{array}$ & $\begin{array}{l}23.3 \\
20.8 \\
19.7 \\
17.8 \\
16.0 \\
11.5 \\
12.7 \\
11.1\end{array}$ & $\begin{array}{l}42.7 \\
43.2 \\
43.5 \\
44.1 \\
44.6 \\
44.9 \\
45.5 \\
46.0\end{array}$ & $\begin{array}{l}41.2 \\
41.8 \\
42.0 \\
42.6 \\
43.3 \\
43.6 \\
44.2 \\
44.8\end{array}$ & $\begin{array}{l}6.6 \\
6.5 \\
5.8 \\
6.0 \\
6.2 \\
6.0 \\
5.7 \\
5.4\end{array}$ & $\begin{array}{l}9.3 \\
9.1 \\
8.0 \\
8.2 \\
8.6 \\
8.3 \\
8.0 \\
7.6\end{array}$ \\
\hline $2007^{p}$ & & 60.7 & 35.2 & 45.1 & 55.8 & 66.2 & 55.0 & 26.0 & 10.3 & 46.5 & 45.4 & 5.2 & 7.3 \\
\hline 2005 & $\begin{array}{l}\text { March } \\
\text { June } \\
\text { Sept } \\
\text { Dec }\end{array}$ & $\begin{array}{r}9.5 \\
19.4 \\
26.1 \\
13.7\end{array}$ & $\begin{array}{l}23.1 \\
46.8 \\
62.3 \\
32.7\end{array}$ & $\begin{array}{l}40.0 \\
43.1 \\
31.1 \\
25.4\end{array}$ & $\begin{array}{l}50.1 \\
73.8 \\
93.7 \\
52.1\end{array}$ & $\begin{array}{r}44.9 \\
91.8 \\
121.6 \\
60.4\end{array}$ & $\begin{array}{r}33.5 \\
70.3 \\
101.0 \\
49.1\end{array}$ & $\begin{array}{l}16.7 \\
33.4 \\
41.9 \\
23.7\end{array}$ & $\begin{array}{l}13.6 \\
12.8 \\
12.5 \\
12.2\end{array}$ & $\begin{array}{l}45.8 \\
45.6 \\
45.0 \\
46.0\end{array}$ & $\begin{array}{l}44.6 \\
44.5 \\
43.8 \\
44.6\end{array}$ & $\begin{array}{l}1.0 \\
1.7 \\
1.9 \\
1.2\end{array}$ & $\begin{array}{r}5.3 \\
9.4 \\
10.6 \\
6.5\end{array}$ \\
\hline 2006 & $\begin{array}{l}\text { March } \\
\text { June } \\
\text { Sept } \\
\text { Dec }\end{array}$ & $\begin{array}{r}8.4 \\
18.3 \\
25.8 \\
12.3\end{array}$ & $\begin{array}{l}20.0 \\
43.3 \\
60.4 \\
28.8\end{array}$ & $\begin{array}{l}27.5 \\
31.1 \\
42.2 \\
38.4\end{array}$ & $\begin{array}{l}34.6 \\
59.9 \\
88.6 \\
49.6\end{array}$ & $\begin{array}{r}36.6 \\
78.8 \\
117.3 \\
52.5\end{array}$ & $\begin{array}{l}28.9 \\
66.3 \\
98.7 \\
43.9\end{array}$ & $\begin{array}{l}15.2 \\
31.9 \\
41.7 \\
21.1\end{array}$ & $\begin{array}{l}11.3 \\
10.7 \\
11.3 \\
11.2\end{array}$ & $\begin{array}{l}46.8 \\
46.2 \\
45.5 \\
46.3\end{array}$ & $\begin{array}{l}45.5 \\
45.1 \\
44.3 \\
45.0\end{array}$ & $\begin{array}{l}0.8 \\
1.7 \\
1.9 \\
1.0\end{array}$ & $\begin{array}{r}4.6 \\
9.4 \\
10.6 \\
5.7\end{array}$ \\
\hline 2007 & $\begin{array}{l}\text { March }{ }^{p} \\
\text { Junee } \\
\text { Septp }^{p} \\
\text { Dec }^{p}\end{array}$ & $\begin{array}{r}8.3 \\
17.1 \\
23.6 \\
11.6\end{array}$ & $\begin{array}{l}19.5 \\
40.0 \\
54.4 \\
26.8\end{array}$ & $\begin{array}{l}25.1 \\
48.2 \\
52.0 \\
54.9\end{array}$ & $\begin{array}{l}35.6 \\
63.6 \\
79.6 \\
44.0\end{array}$ & $\begin{array}{r}36.3 \\
74.3 \\
107.3 \\
46.4\end{array}$ & $\begin{array}{l}29.0 \\
60.9 \\
89.1 \\
40.4\end{array}$ & $\begin{array}{l}14.9 \\
30.1 \\
38.5 \\
20.4\end{array}$ & $\begin{array}{l}10.5 \\
10.2 \\
10.5 \\
9.8\end{array}$ & $\begin{array}{l}47.1 \\
46.6 \\
46.0 \\
47.0\end{array}$ & $\begin{array}{l}45.8 \\
45.6 \\
44.9 \\
45.8\end{array}$ & $\begin{array}{l}0.8 \\
1.6 \\
1.8 \\
1.0\end{array}$ & $\begin{array}{l}4.5 \\
9.0 \\
9.9 \\
5.7\end{array}$ \\
\hline $\begin{array}{l}\text { Females } \\
1961 \\
1966 \\
1971 \\
1976 \\
1981\end{array}$ & & $\begin{array}{l}18.0 \\
25.1 \\
39.6 \\
65.1 \\
75.1\end{array}$ & $\begin{array}{r}97.1 \\
114.7 \\
134.0 \\
122.2 \\
90.7\end{array}$ & $\begin{array}{l}542.2 \\
567.8 \\
464.4 \\
458.9 \\
257.5\end{array}$ & $\begin{array}{l}409.6 \\
411.2 \\
359.0 \\
272.3 \\
202.1\end{array}$ & $\begin{array}{l}250.2 \\
254.8 \\
232.7 \\
188.0 \\
142.9\end{array}$ & $\begin{array}{r}111.5 \\
135.9 \\
139.8 \\
124.0 \\
95.5\end{array}$ & $\begin{array}{l}35.6 \\
37.8 \\
49.3 \\
40.9 \\
29.0\end{array}$ & $\begin{array}{l}46.8 \\
52.4 \\
57.0 \\
59.8 \\
57.9\end{array}$ & $\begin{array}{l}37.2 \\
36.2 \\
35.7 \\
34.9 \\
35.1\end{array}$ & $\begin{array}{l}35.9 \\
34.3 \\
33.0 \\
32.4 \\
33.4\end{array}$ & $\begin{array}{l}16.5 \\
16.8 \\
17.7 \\
17.0 \\
13.5\end{array}$ & $\begin{array}{l}6.5 \\
6.3 \\
6.3 \\
5.9 \\
4.6\end{array}$ \\
\hline $\begin{array}{l}1986 \\
1991\end{array}$ & & $\begin{array}{l}80.0 \\
73.4\end{array}$ & $\begin{array}{l}68.7 \\
50.3\end{array}$ & $\begin{array}{l}190.9 \\
111.9\end{array}$ & $\begin{array}{l}155.9 \\
118.1\end{array}$ & $\begin{array}{r}111.6 \\
89.7\end{array}$ & $\begin{array}{l}75.6 \\
55.3\end{array}$ & $\begin{array}{l}24.4 \\
20.9\end{array}$ & $\begin{array}{l}51.2 \\
47.4\end{array}$ & $\begin{array}{l}36.0 \\
37.1\end{array}$ & $\begin{array}{l}34.7 \\
35.7\end{array}$ & $\begin{array}{r}11.2 \\
8.6\end{array}$ & $\begin{array}{l}3.8 \\
2.9\end{array}$ \\
\hline $\begin{array}{l}1994 \\
1995 \\
1996 \\
1997 \\
1998\end{array}$ & & $\begin{array}{l}76.9 \\
76.9 \\
78.9 \\
77.1 \\
73.3\end{array}$ & $\begin{array}{l}47.3 \\
45.7 \\
45.6 \\
43.3 \\
40.1\end{array}$ & $\begin{array}{l}167.3 \\
166.5 \\
183.5 \\
188.5 \\
175.0\end{array}$ & $\begin{array}{l}121.0 \\
118.8 \\
120.6 \\
119.4 \\
114.5\end{array}$ & $\begin{array}{l}91.4 \\
91.9 \\
93.6 \\
90.8 \\
87.1\end{array}$ & $\begin{array}{l}54.4 \\
54.8 \\
56.0 \\
54.6 \\
52.2\end{array}$ & $\begin{array}{l}20.6 \\
19.8 \\
20.4 \\
19.6 \\
18.4\end{array}$ & $\begin{array}{l}44.4 \\
42.8 \\
40.8 \\
39.0 \\
37.1\end{array}$ & $\begin{array}{l}37.9 \\
38.1 \\
38.6 \\
38.9 \\
39.3\end{array}$ & $\begin{array}{l}36.3 \\
36.6 \\
37.1 \\
37.4 \\
37.9\end{array}$ & $\begin{array}{l}7.9 \\
7.5 \\
7.3 \\
7.0 \\
6.6\end{array}$ & $\begin{array}{l}2.7 \\
2.6 \\
2.6 \\
2.5 \\
2.4\end{array}$ \\
\hline $\begin{array}{l}1999 \\
2000 \\
2001 \\
2002 \\
2003 \\
2004 \\
2005 \\
2006\end{array}$ & & $\begin{array}{l}72.0 \\
74.1 \\
66.1 \\
69.2 \\
73.1 \\
72.9 \\
65.9 \\
61.0\end{array}$ & $\begin{array}{l}38.4 \\
38.5 \\
33.5 \\
34.1 \\
35.0 \\
34.0 \\
30.0 \\
27.4\end{array}$ & $\begin{array}{r}155.0 \\
137.8 \\
104.6 \\
109.8 \\
118.1 \\
12.7 \\
75.4 \\
68.9\end{array}$ & $\begin{array}{r}107.0 \\
107.5 \\
96.9 \\
100.1 \\
101.7 \\
95.6 \\
76.7 \\
69.2\end{array}$ & $\begin{array}{l}84.8 \\
85.6 \\
79.3 \\
80.8 \\
83.3 \\
82.7 \\
72.2 \\
67.8\end{array}$ & $\begin{array}{l}52.3 \\
54.2 \\
48.5 \\
50.8 \\
52.4 \\
52.6 \\
47.6 \\
44.3\end{array}$ & $\begin{array}{l}17.8 \\
18.4 \\
15.9 \\
16.8 \\
18.3 \\
18.0 \\
16.8 \\
15.8\end{array}$ & $\begin{array}{l}34.7 \\
32.0 \\
30.7 \\
28.2 \\
26.1 \\
24.0 \\
21.2 \\
19.6\end{array}$ & $\begin{array}{l}39.7 \\
40.1 \\
40.4 \\
40.9 \\
41.5 \\
41.9 \\
42.6 \\
43.0\end{array}$ & $\begin{array}{l}38.3 \\
38.9 \\
39.2 \\
39.7 \\
40.3 \\
40.8 \\
41.6 \\
42.1\end{array}$ & $\begin{array}{l}6.2 \\
6.2 \\
5.6 \\
5.7 \\
5.9 \\
5.8 \\
5.4 \\
5.2\end{array}$ & $\begin{array}{l}2.3 \\
2.3 \\
2.0 \\
2.1 \\
2.2 \\
2.2 \\
2.1 \\
2.0\end{array}$ \\
\hline $2007^{\mathrm{P}}$ & & 58.1 & 25.4 & 66.4 & 67.0 & 64.4 & 41.7 & 15.1 & 18.4 & 43.5 & 42.7 & 49.9 & 2.0 \\
\hline 2005 & $\begin{array}{l}\text { March } \\
\text { June } \\
\text { Sept } \\
\text { Dec }\end{array}$ & $\begin{array}{r}9.6 \\
18.3 \\
24.5 \\
13.5\end{array}$ & $\begin{array}{l}17.6 \\
33.4 \\
44.2 \\
24.5\end{array}$ & $\begin{array}{l}75.7 \\
79.9 \\
86.1 \\
59.1\end{array}$ & $\begin{array}{r}58.2 \\
78.8 \\
108.7 \\
60.6\end{array}$ & $\begin{array}{r}43.3 \\
77.8 \\
109.4 \\
58.0\end{array}$ & $\begin{array}{l}27.2 \\
52.0 \\
72.2 \\
38.7\end{array}$ & $\begin{array}{r}9.6 \\
19.7 \\
24.0 \\
13.9\end{array}$ & $\begin{array}{l}23.6 \\
20.2 \\
21.2 \\
20.8\end{array}$ & $\begin{array}{l}42.2 \\
43.0 \\
42.4 \\
42.7\end{array}$ & $\begin{array}{l}41.2 \\
42.0 \\
41.5 \\
41.6\end{array}$ & $\begin{array}{l}0.8 \\
1.5 \\
1.9 \\
1.2\end{array}$ & $\begin{array}{l}1.3 \\
2.3 \\
2.9 \\
1.8\end{array}$ \\
\hline 2006 & $\begin{array}{l}\text { March } \\
\text { June } \\
\text { Sept } \\
\text { Dec }\end{array}$ & $\begin{array}{r}8.2 \\
17.3 \\
24.1 \\
11.8\end{array}$ & $\begin{array}{l}14.8 \\
30.9 \\
42.7 \\
20.9\end{array}$ & $\begin{array}{r}40.5 \\
75.6 \\
101.6 \\
57.5\end{array}$ & $\begin{array}{r}43.4 \\
73.3 \\
103.8 \\
55.7\end{array}$ & $\begin{array}{r}38.8 \\
72.7 \\
106.9 \\
52.1\end{array}$ & $\begin{array}{l}22.7 \\
49.4 \\
70.7 \\
33.8\end{array}$ & $\begin{array}{r}8.6 \\
18.6 \\
24.1 \\
12.0\end{array}$ & $\begin{array}{l}21.3 \\
18.5 \\
19.5 \\
20.0\end{array}$ & $\begin{array}{l}43.1 \\
43.4 \\
42.8 \\
43.0\end{array}$ & $\begin{array}{l}41.8 \\
42.4 \\
42.0 \\
42.0\end{array}$ & $\begin{array}{l}0.8 \\
1.5 \\
1.8 \\
1.0\end{array}$ & $\begin{array}{l}1.3 \\
2.4 \\
2.8 \\
1.6\end{array}$ \\
\hline 2007 & $\begin{array}{l}\text { March } \\
\text { June } \\
\text { Sept卢 } \\
\text { Dec }^{p}\end{array}$ & $\begin{array}{r}8.0 \\
16.4 \\
22.1 \\
11.5\end{array}$ & $\begin{array}{l}14.2 \\
28.8 \\
38.4 \\
20.0\end{array}$ & $\begin{array}{l}40.7 \\
67.4 \\
85.9 \\
71.0\end{array}$ & $\begin{array}{l}41.9 \\
70.8 \\
97.9 \\
57.1\end{array}$ & $\begin{array}{l}35.7 \\
70.5 \\
98.1 \\
52.8\end{array}$ & $\begin{array}{l}22.6 \\
46.3 \\
65.1 \\
32.4\end{array}$ & $\begin{array}{r}8.6 \\
17.9 \\
22.2 \\
11.8\end{array}$ & $\begin{array}{l}19.0 \\
177.6 \\
18.2 \\
19.6\end{array}$ & $\begin{array}{l}43.7 \\
43.8 \\
43.2 \\
43.4\end{array}$ & $\begin{array}{l}42.8 \\
43.1 \\
42.5 \\
42.5\end{array}$ & $\begin{array}{l}0.8 \\
1.5 \\
1.7 \\
1.0\end{array}$ & $\begin{array}{l}1.2 \\
2.4 \\
2.8 \\
1.5\end{array}$ \\
\hline
\end{tabular}

Note: Rates have been revised from 2002, to include the adjustments for marriages to England and Wales residents occuring abroad - see 'In Brief'. 1 Figures for all marriages can be found in Table 2.1.

2 Per 1,000 divorced persons aged 16 and over.

2 Per 1,000 divorced persons aged 16 and over.

4 Per 1,000 widowed persons aged 16 and over.

p provisional 


\begin{tabular}{|c|c|c|c|c|c|c|c|c|c|c|c|c|c|}
\hline \multirow{2}{*}{$\begin{array}{l}\text { Table } 9.3 \\
\text { England and Wales }\end{array}$} & \multicolumn{13}{|c|}{ Divorces: age and sex } \\
\hline & \multicolumn{13}{|c|}{ Numbers (thousands), rates, percentages, mean and median age } \\
\hline \multirow{3}{*}{$\begin{array}{l}\text { Year and } \\
\text { quarter }\end{array}$} & \multirow{2}{*}{$\begin{array}{l}\text { Petitions } \\
\text { filed }\end{array}$} & \multicolumn{3}{|c|}{ Decrees made absolute } & \multicolumn{6}{|c|}{ Divorce decrees per 1,000 married population } & \multirow{2}{*}{$\begin{array}{c}\text { Per cent } \\
\text { aged } \\
\text { under } 35\end{array}$} & \multirow{2}{*}{$\begin{array}{l}\text { Mean age } \\
\text { at divorce' }\end{array}$} & \multirow{2}{*}{$\begin{array}{l}\text { Median } \\
\text { age at } \\
\text { divorce' }^{1}\end{array}$} \\
\hline & & $\begin{array}{l}\text { All } \\
\text { divorces }\end{array}$ & $\begin{array}{c}1 \mathrm{1st} \\
\text { marriage }\end{array}$ & $\begin{array}{c}\text { 2nd or } \\
\text { later } \\
\text { marriage }\end{array}$ & $\begin{array}{c}16 \text { and } \\
\text { over }\end{array}$ & $16-24$ & $25-29$ & 30-34 & $35-44$ & $\begin{array}{l}45 \text { and } \\
\text { over }\end{array}$ & & & \\
\hline & \multicolumn{4}{|c|}{ Numbers } & & & & & & & & & \\
\hline $\begin{array}{l}\text { Males } \\
1961 \\
1966 \\
1971 \\
1976 \\
1981\end{array}$ & $\begin{array}{l}13.7 \\
18.3 \\
44.2 \\
43.3 \\
46.7\end{array}$ & $\begin{array}{r}25.4 \\
39.1 \\
74.4 \\
126.5 \\
145.7\end{array}$ & $\begin{array}{r}23.5 \\
36.4 \\
69.3 \\
615.7 \\
127.6\end{array}$ & $\begin{array}{r}1.9 \\
2.7 \\
51.2 \\
11.0 \\
18.1\end{array}$ & $\begin{array}{r}2.1 \\
3.2 \\
5.9 \\
10.1 \\
11.9\end{array}$ & $\begin{array}{r}1.4 \\
2.6 \\
5.0 \\
13.6\end{array}$ & $\begin{array}{r}3.9 \\
6.8 \\
12.5 \\
22.4 \\
27.6\end{array}$ & $\begin{aligned} & 4.1 \\
& 11.8 \\
& 11.8 \\
& 18.9 \\
& 2.8\end{aligned}$ & $\begin{array}{r}3.1 \\
4.5 \\
7.9 \\
14.1 \\
17.0\end{array}$ & $\begin{array}{l}1.1 \\
1.5 \\
3.1 \\
4.5 \\
4.8\end{array}$ & $\begin{array}{l}38.3 \\
44.2 \\
44.8 \\
48.6 \\
486\end{array}$ & $\begin{array}{l}38.6 \\
39.4 \\
38.0 \\
37.7\end{array}$ & $\begin{array}{l}36.4 \\
36.6 \\
35.4 \\
35.4\end{array}$ \\
\hline $\begin{array}{l}1986 \\
1991 \\
1996\end{array}$ & $\begin{aligned} 49.7 \\
. .\end{aligned}$ & $\begin{array}{l}153.9 \\
58.7 \\
57.1\end{array}$ & $\begin{array}{l}128.0 \\
129.8 \\
125.8\end{array}$ & $\begin{array}{l}25.9 \\
29.0 \\
31.3\end{array}$ & $\begin{array}{l}13.0 \\
13.6 \\
1.39\end{array}$ & $\begin{array}{l}31.4 \\
26.1 \\
28.1\end{array}$ & $\begin{array}{l}31.4 \\
32.4\end{array}$ & $\begin{array}{l}25.2 \\
28.6 \\
33.6\end{array}$ & $\begin{array}{l}18.0 \\
20.2\end{array}$ & $\begin{array}{l}5.2 \\
5.6\end{array}$ & $\begin{array}{l}45.6 \\
42.7 \\
32.5\end{array}$ & $\begin{array}{l}37.8 \\
38.6 \\
38.6\end{array}$ & $\begin{array}{l}36.2 \\
37.0 \\
32.1\end{array}$ \\
\hline 1997 & 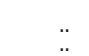 & $\begin{array}{l}146.7 \\
465.2\end{array}$ & $\begin{array}{l}117.3 \\
116.0\end{array}$ & $\begin{array}{l}29.4 \\
29.2\end{array}$ & $\begin{array}{l}13.1 \\
13.0\end{array}$ & $\begin{array}{l}26.0 \\
25.8\end{array}$ & $\begin{array}{l}30.4 \\
30.7\end{array}$ & $\begin{array}{l}28.7 \\
28.4\end{array}$ & 21.1 & $\begin{array}{l}6.1 \\
6.1\end{array}$ & $\begin{array}{l}35.9 \\
34.3\end{array}$ & $\begin{array}{l}40.2 \\
40.4\end{array}$ & $\begin{array}{l}38.4 \\
38.7\end{array}$ \\
\hline 1999 &.. & & & & & & & & 21.9 & 6.3 & 32.1 & 40.9 & 39.2 \\
\hline $\begin{array}{l}2000 \\
2001 \\
20003\end{array}$ & $\begin{array}{l}\ddot{.} \\
. .\end{array}$ & $\begin{array}{l}143.1 \\
143.8 \\
143.7\end{array}$ & $\begin{array}{l}114.1 \\
116.9 \\
121.4\end{array}$ & $\begin{array}{l}29.1 \\
29.5 \\
30.8 \\
32.0\end{array}$ & $\begin{array}{l}12.1 \\
13.0 \\
13.4 \\
14.0\end{array}$ & $\begin{array}{l}22.3 \\
22.3 \\
22.8\end{array}$ & $\begin{array}{l}27.9 \\
28.9 \\
28.9\end{array}$ & $\begin{array}{l}28.4 \\
28.3 \\
29.9\end{array}$ & $\begin{array}{l}22.9 \\
22.8 \\
25.0\end{array}$ & $\begin{array}{l}6.3 \\
6.5 \\
6.9\end{array}$ & $\begin{array}{l}28.9 \\
26.7 \\
24.7\end{array}$ & $\begin{array}{l}41.5 \\
41.9 \\
42.3\end{array}$ & $\begin{array}{l}49.0 \\
40.0 \\
40.9\end{array}$ \\
\hline $\begin{array}{l}2004 \\
2005 \\
20067^{p}\end{array}$ & $\begin{array}{l}. . \\
. . \\
.\end{array}$ & $\begin{array}{l}155.4 \\
131.8 \\
132.6 \\
128.5\end{array}$ & $\begin{array}{l}121.1 \\
11.7 \\
04.3 \\
001.7\end{array}$ & $\begin{array}{l}32.3 \\
30.1 \\
28.2 \\
26.9\end{array}$ & $\begin{array}{l}14.0 \\
13.0 \\
12.2 \\
11.8\end{array}$ & $\begin{array}{l}23.0 \\
21.6 \\
19.5 \\
19.2\end{array}$ & $\begin{array}{l}28.5 \\
26.0 \\
244.5 \\
24.0\end{array}$ & $\begin{array}{l}29.1 \\
26.0 \\
24.3 \\
23.6\end{array}$ & $\begin{array}{l}25.1 \\
23.2 \\
21.8 \\
21.1\end{array}$ & $\begin{array}{l}7.7 \\
7.4 \\
7.1\end{array}$ & $\begin{array}{l}23.3 \\
21.8 \\
20.6 \\
20.0\end{array}$ & $\begin{array}{l}42.7 \\
43.7 \\
43.4 \\
43.7\end{array}$ & $\begin{array}{l}41.4 \\
42.0 \\
42.4 \\
42.7\end{array}$ \\
\hline $\begin{array}{c}2005 \text { March } \\
\text { Junet } \\
\text { Sept } \\
\text { Dec }\end{array}$ & $\ddot{.:}$ & $\begin{array}{l}36.2 \\
36.5 \\
35.6 \\
33.4\end{array}$ & $\begin{array}{l}28.5 \\
28.7 \\
28.0 \\
26.4\end{array}$ & $\begin{array}{l}7.7 \\
77.8 \\
7.0\end{array}$ & $\begin{array}{l}13.4 \\
13.4 \\
12: .9 \\
12.1\end{array}$ & $\begin{array}{l}23.3 \\
22.1 \\
21.2 \\
19.7\end{array}$ & $\begin{array}{l}26.9 \\
26.4 \\
25.9 \\
24.8\end{array}$ & $\begin{array}{l}27.6 \\
27.6 \\
23.3 \\
23.6\end{array}$ & $\begin{array}{l}24.1 \\
23.8 \\
23.2 \\
21.6\end{array}$ & $\begin{array}{l}7.6 \\
77.8 \\
7.4\end{array}$ & $\begin{array}{l}22.2 \\
22.5 \\
21.9 \\
21.4\end{array}$ & $\begin{array}{l}43.0 \\
43.2 \\
43.0 \\
43.1\end{array}$ & $\begin{array}{l}41.8 \\
42.1 \\
41.9 \\
42.1\end{array}$ \\
\hline 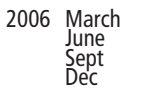 & $\begin{array}{l}. . \\
. .\end{array}$ & $\begin{array}{l}34.3 \\
32.9 \\
32.9 \\
32.4\end{array}$ & $\begin{array}{l}27.0 \\
25.8 \\
26.0 \\
25.4\end{array}$ & $\begin{array}{l}7.3 \\
77.1 \\
6.9 \\
7.0\end{array}$ & $\begin{array}{l}12.8 \\
12.1 \\
12.0 \\
11.8\end{array}$ & $\begin{array}{l}21.9 \\
19.7 \\
19.0 \\
17.5\end{array}$ & $\begin{array}{l}25.6 \\
24.4 \\
23.9 \\
24.1\end{array}$ & $\begin{array}{l}26.0 \\
24.1 \\
23.7 \\
23.5\end{array}$ & $\begin{array}{l}23.1 \\
211.6 \\
21.6 \\
20.9\end{array}$ & $\begin{array}{l}7.4 \\
7.2 \\
7.1 \\
7.0\end{array}$ & $\begin{array}{l}20.9 \\
20.6 \\
20.4 \\
20.6\end{array}$ & $\begin{array}{l}43.3 \\
43.4 \\
43.4 \\
43.5\end{array}$ & $\begin{array}{l}42.2 \\
42.2 \\
42.3 \\
42.5\end{array}$ \\
\hline $2007 \begin{array}{c}\text { Marchp } \\
\text { June } \\
\text { Septp } \\
\text { Sept } \\
\text { Dec }\end{array}$ & $\begin{array}{l}. . \\
.:\end{array}$ & $\begin{array}{l}34.7 \\
33.2 \\
33.0 \\
27.6\end{array}$ & $\begin{array}{l}27.4 \\
26.3 \\
26.1 \\
21.9\end{array}$ & $\begin{array}{l}7.3 \\
6.9 \\
6.9 \\
55.7\end{array}$ & $\begin{array}{l}13.0 \\
12.3 \\
12.1 \\
10.1\end{array}$ & $\begin{array}{l}21.6 \\
20.3 \\
21.1 \\
13.9\end{array}$ & $\begin{array}{l}26.4 \\
25.0 \\
25.0 \\
19.8\end{array}$ & $\begin{array}{l}26.6 \\
24.3 \\
24.1 \\
19.7\end{array}$ & $\begin{array}{l}23.2 \\
22.0 \\
21.6 \\
17.7\end{array}$ & $\begin{array}{l}7.7 \\
7.3 \\
7.2 \\
6.3\end{array}$ & $\begin{array}{l}20.4 \\
19.9 \\
20.2 \\
19.3\end{array}$ & $\begin{array}{l}\begin{array}{r}43.6 \\
43.6 \\
43.6 \\
43.9\end{array} \\
43.9\end{array}$ & $\begin{array}{l}42.6 \\
\text { 站.6. } \\
\text { a2.6. } \\
43.0\end{array}$ \\
\hline $\begin{array}{l}2008^{2} \text { Marchp } \\
\text { June } \\
\text { Suntp }\end{array}$ & $\ddot{.}$ & $\begin{array}{l}31.9 \\
30.8 \\
30.8\end{array}$ & $\begin{array}{l}25.2 \\
24.4 \\
24.4\end{array}$ & $\begin{array}{l}6.7 \\
6.4\end{array}$ & 11.8 & $\begin{array}{l}17.6 \\
15.3 \\
15.9\end{array}$ & $\begin{array}{l}23.9 \\
22.8 \\
22.8\end{array}$ & $\begin{array}{l}22.5 \\
22.0\end{array}$ & $\begin{array}{l}20.7 \\
19.8\end{array}$ & 7.42 & 19.3 & $\begin{array}{l}43.9 \\
44.0\end{array}$ & $\begin{array}{l}43.1 \\
43.1\end{array}$ \\
\hline $\begin{array}{l}\text { Females } \\
\text { Fina }\end{array}$ & & & & & & & & & & & & & \\
\hline $\begin{array}{l}1960 \\
1966 \\
1971 \\
1981\end{array}$ & $\begin{array}{r}18.2 \\
26.3 \\
10.7 \\
10.5 \\
123.5\end{array}$ & $\begin{array}{r}25.4 \\
39.1 \\
126.4 \\
125.7\end{array}$ & $\begin{array}{r}23.4 \\
36.2 \\
69.3 \\
115.9 \\
127.7\end{array}$ & $\begin{aligned} 2.0 \\
2.8 \\
50.1 \\
10.8 \\
18.0\end{aligned}$ & $\begin{array}{r}2.1 \\
3.2 \\
50.9 \\
11.9\end{array}$ & $\begin{array}{r}2.44 \\
7.1 \\
14.5 \\
22.3\end{array}$ & $\begin{array}{r}4.5 \\
1.6 \\
13.0 \\
20.4 \\
26.7\end{array}$ & $\begin{array}{r}3.8 \\
1.0 . \\
10.5 \\
128.3 \\
20.2\end{array}$ & $\begin{array}{r}2.7 \\
3.9 \\
6.7 \\
12.6 \\
14.9\end{array}$ & $\begin{array}{l}0.9 \\
1.2 \\
2.8 \\
4.0 \\
3.9\end{array}$ & $\begin{array}{l}45.3 \\
54.7 \\
55.4 \\
58.6 \\
58.0\end{array}$ & $\begin{array}{l}35.8 \\
36.8 \\
36.0 \\
35.2\end{array}$ & $\begin{array}{l}33.6 \\
33.6 \\
33.1 \\
33.2\end{array}$ \\
\hline 1986 & 130.7 & $\begin{array}{l}153.9 \\
1558.7\end{array}$ & $\begin{array}{l}128.8 \\
130.9\end{array}$ & $\begin{aligned} 25.1 \\
27.8\end{aligned}$ & $\begin{array}{l}12.8 \\
13.4\end{array}$ & $\begin{array}{l}30.7 \\
28.7\end{array}$ & $\begin{array}{l}28.6 \\
30.7\end{array}$ & $\begin{array}{l}22.0 \\
25.0\end{array}$ & 15.8 & $\begin{array}{l}4.1 \\
4.5\end{array}$ & $\begin{array}{l}55.0 \\
52.7\end{array}$ & $\begin{array}{l}35.3 \\
36.0\end{array}$ & $\begin{array}{l}33.6 \\
34.3\end{array}$ \\
\hline & 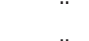 & & & 28.4 & 129 & $\begin{array}{l}38.1 \\
280\end{array}$ & 31.3 & 26.3 & & 4.1 & 45.9 & 5.5 & \\
\hline $\begin{array}{l}1998 \\
1999\end{array}$ & $\ddot{\ddot{\prime}}$ & $\begin{array}{l}145.1 \\
145.2 \\
144.6\end{array}$ & $\begin{array}{l}110.8 \\
115.8\end{array}$ & $\begin{array}{l}28.4 \\
28.5 \\
29.1\end{array}$ & $\begin{array}{l}12.9 \\
12.9\end{array}$ & $\begin{array}{l}28.5 \\
25.6\end{array}$ & $\begin{array}{l}31.3 \\
30.6 \\
30.6\end{array}$ & $\begin{array}{l}26.3 \\
26.6\end{array}$ & $\begin{array}{l}18.9 \\
19.9\end{array}$ & $\begin{array}{l}4.9 \\
5.1\end{array}$ & $\begin{array}{l}44.3 \\
41.7\end{array}$ & $\begin{array}{l}31.9 \\
38.4\end{array}$ & $\begin{array}{l}36.0 \\
36.3 \\
36.9\end{array}$ \\
\hline $\begin{array}{l}2000 \\
2001\end{array}$ & $\ddot{.}$ & $\begin{array}{l}141.1 \\
143.8\end{array}$ & 112.6 & $\begin{array}{r}28.5 \\
29.2\end{array}$ & $\begin{array}{l}12.6 \\
12.9 \\
13.2\end{array}$ & $\begin{array}{l}24.5 \\
23.9\end{array}$ & $\begin{array}{l}29.0 \\
229.2\end{array}$ & $\begin{array}{l}26.6 \\
27.6\end{array}$ & $\begin{array}{l}19.4 \\
20.5\end{array}$ & $\begin{array}{l}5.2 \\
5.4\end{array}$ & $\begin{array}{r}39.6 \\
37.8 \\
35.9\end{array}$ & $\begin{array}{l}38.8 \\
39.1\end{array}$ & $\begin{array}{l}37.3 \\
37.7 \\
38.7\end{array}$ \\
\hline $\begin{array}{l}2002 \\
2003 \\
2004\end{array}$ & $\ddot{.}$ & $\begin{array}{l}154.7 \\
53.5 \\
153.4\end{array}$ & $\begin{array}{l}11.5 \\
121.9 \\
121.8\end{array}$ & $\begin{aligned} 30.2 \\
31.6 \\
31.6\end{aligned}$ & $\begin{array}{l}13.3 \\
13.9 \\
1.0\end{array}$ & $\begin{array}{l}26.3 \\
27.3 \\
26.7\end{array}$ & $\begin{array}{l}30.0 \\
30.9 \\
30.9\end{array}$ & $\begin{array}{l}28.2 \\
28.9 \\
28.6\end{array}$ & $\begin{array}{l}22.6 \\
23.2 \\
23.5\end{array}$ & $\begin{array}{l}5.1 \\
6.4\end{array}$ & $\begin{array}{l}33.9 \\
33.7 \\
32.9\end{array}$ & $\begin{array}{l}39.4 \\
39.8 \\
40.2\end{array}$ & $\begin{array}{l}38.2 \\
38.7 \\
39.2\end{array}$ \\
\hline $\begin{array}{l}2005 \\
20067^{p}\end{array}$ & $\ddot{\ddot{\prime}}$ & $\begin{array}{l}141.8 \\
128.6 \\
128.5\end{array}$ & $\begin{array}{l}120.0 \\
102.8\end{array}$ & $\begin{array}{l}29.7 \\
26.5\end{array}$ & $\begin{array}{l}13.0 \\
12.2 \\
11.8\end{array}$ & $\begin{array}{l}253.3 \\
23.0 \\
23.0\end{array}$ & $\begin{array}{l}27.6 \\
26.9 \\
26.0\end{array}$ & $\begin{array}{l}25.7 \\
23.9 \\
23.7\end{array}$ & $\begin{array}{l}22.1 \\
20.9 \\
20.3\end{array}$ & $\begin{array}{l}6.2 \\
6.0 \\
6.1\end{array}$ & $\begin{array}{l}32.0 \\
27.8 \\
27.8\end{array}$ & $\begin{array}{l}40.6 \\
40.9 \\
41.2\end{array}$ & $\begin{array}{l}39.8 \\
40.1 \\
40.5\end{array}$ \\
\hline 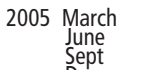 & 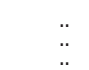 & $\begin{array}{l}36.2 \\
36.5 \\
35.6\end{array}$ & $\begin{array}{l}28.6 \\
28.8 \\
28.1\end{array}$ & $\begin{array}{l}7.6 \\
7.7 \\
7.5\end{array}$ & $\begin{array}{l}13.4 \\
13.4 \\
12.9\end{array}$ & $\begin{array}{l}25.9 \\
26.3 \\
25.8\end{array}$ & $\begin{array}{l}28.6 \\
27.3 \\
27.8\end{array}$ & $\begin{array}{l}27.0 \\
26.9 \\
25.8\end{array}$ & $\begin{array}{r}22.9 \\
22.8 \\
22.8\end{array}$ & $\begin{array}{l}6.4 \\
6.5 \\
6.1\end{array}$ & $\begin{array}{l}30.2 \\
29.8 \\
33.2\end{array}$ & $\begin{array}{l}40.6 \\
40.7 \\
40.7\end{array}$ & $\begin{array}{l}39.6 \\
39.9 \\
39.9\end{array}$ \\
\hline Dec &. & 33.4 & $\begin{array}{l}28.5 \\
26.5\end{array}$ & 6.9 & 12.1 & 23.2 & 26.7 & 23.2 & 20.7 & 5.9 & $\begin{array}{l}39.5 \\
29.7\end{array}$ & 40.6 & 39.8 \\
\hline $2006 \begin{array}{c}\text { March } \\
\text { June } \\
\text { Sent }\end{array}$ & $\ddot{.}$ & $\begin{array}{r}34.3 \\
32.9 \\
32.9\end{array}$ & $\begin{array}{l}27.2 \\
25.9 \\
26.9\end{array}$ & $\begin{array}{l}7.1 \\
7.0 \\
6.8\end{array}$ & $\begin{array}{l}12.8 \\
12.1 \\
120\end{array}$ & $\begin{array}{l}25.0 \\
22.2\end{array}$ & $\begin{array}{l}28.0 \\
26.7\end{array}$ & $\begin{array}{l}25.5 \\
23.4 \\
23.4\end{array}$ & $\begin{array}{l}22.2 \\
21.0\end{array}$ & $\begin{array}{l}6.2 \\
6.0\end{array}$ & $\begin{array}{l}29.0 \\
28.3 \\
288\end{array}$ & $\begin{array}{l}40.8 \\
40.9\end{array}$ & $\begin{array}{l}40.0 \\
40.1\end{array}$ \\
\hline Dec & $\ddot{*}$ & 32.4 & 25.5 & 6.8 & 11.8 & 23.5 & 26.6 & 22.9 & 20.0 & $\begin{array}{l}6.0 \\
5.9\end{array}$ & 28.9 & 40.9 & 40.2 \\
\hline $2007 \begin{array}{c}\text { Marchp } \\
\text { Junue } \\
\text { Sepet }\end{array}$ & $\ddot{.}$ & $\begin{array}{l}34.7 \\
33.2 \\
33.0\end{array}$ & $\begin{array}{l}27.5 \\
27.2 \\
26.3\end{array}$ & $\begin{array}{l}7.2 \\
6.9 \\
6.7\end{array}$ & $\begin{array}{l}13.0 \\
12.3 \\
12.1\end{array}$ & $\begin{array}{l}27.5 \\
23.0 \\
24.0\end{array}$ & $\begin{array}{l}28.4 \\
227.5 \\
27.0\end{array}$ & $\begin{array}{l}26.4 \\
24.2 \\
24.4\end{array}$ & $\begin{array}{l}22.2 \\
21.1 \\
20.7\end{array}$ & $\begin{array}{l}6.5 \\
6.2 \\
6.1\end{array}$ & $\begin{array}{l}28.4 \\
27.8 \\
28.2\end{array}$ & $\begin{array}{l}41.1 \\
41.2 \\
41.1\end{array}$ & $\begin{array}{l}40.4 \\
40.5 \\
40.4\end{array}$ \\
\hline Deces & :" & 27.6 & 22.0 & 5.6 & 10.1 & 17.5 & 21.3 & 19.7 & 17.2 & 5.4 & 26.8 & 41.5 & 40.9 \\
\hline $\begin{array}{c}2008^{2} \begin{array}{c}\text { Marcchp } \\
\text { June } \\
\text { Sept }\end{array} \\
\text { Sept }\end{array}$ & $\begin{array}{l}. . \\
. .\end{array}$ & $\begin{array}{l}31.9 \\
30.8 \\
30.4\end{array}$ & $\begin{array}{l}25.3 \\
24.5 \\
24.2\end{array}$ & $\begin{array}{l}6.7 \\
6.3 \\
6.2\end{array}$ & $\begin{array}{l}11.8 \\
11.4\end{array}$ & $\begin{array}{l}22.7 \\
20.6 \\
21.5\end{array}$ & $\begin{array}{l}25.9 \\
25.5 \\
25.7\end{array}$ & $\begin{array}{l}23.0 \\
211.5 \\
21.7\end{array}$ & $\begin{array}{l}19.9 \\
19.3 \\
18.4\end{array}$ & $\begin{array}{l}6.3 \\
6.1 \\
6.0\end{array}$ & $\begin{array}{l}27.4 \\
26.9 \\
27.9\end{array}$ & $\begin{array}{l}41.4 \\
41.6 \\
41.4\end{array}$ & $\begin{array}{l}40.8 \\
41.0 \\
40.9\end{array}$ \\
\hline
\end{tabular}

Note: Rates have been revised from 2002, to include the adjustments for marriages to England and Wales residents occuring abroad - see 'In Brief'.

1 The mean/median ages shown in this table are unstandardised and therefore take no account of changes in the structure of the population by age or marital status.

Rates for 2008 are based on 2007 marital status estimates.

Rates for 2008 ar
provisional.

\section{Divorce petitions entered by year and quarter 1996-2008}

England and Wales

\begin{tabular}{|c|c|c|c|c|c|c|c|c|c|}
\hline \multicolumn{9}{|c|}{ England and Wales } & Numbers (thousands \\
\hline Year & March Qtr & June Qtr & Sept Qtr & Dec Qtr & Year & March Qtr & June Qtr & Sept Qtr & Dec Qtr \\
\hline $\begin{array}{l}1996 \\
1997 \\
1998 \\
1999 \\
2000 \\
2001 \\
2002\end{array}$ & $\begin{array}{l}45.6 \\
35.6 \\
43.0 \\
41.4 \\
39.3 \\
45.4 \\
45.4\end{array}$ & $\begin{array}{l}44.5 \\
43.7 \\
40.3 \\
39.5 \\
37.6 \\
42.6 \\
44.3\end{array}$ & $\begin{array}{l}45.3 \\
44.0 \\
42.1 \\
41.3 \\
39.5 \\
42.9 \\
45.4\end{array}$ & $\begin{array}{l}43.4 \\
40.9 \\
41.0 \\
40.5 \\
41.8 \\
42.0 \\
42.6\end{array}$ & $\begin{array}{l}2003 \\
2004 \\
2005 \\
2006 \\
2007 \\
2008^{\mathrm{P}}\end{array}$ & $\begin{array}{l}46.3 \\
45.5 \\
38.0 \\
38.8 \\
38.5 \\
33.3\end{array}$ & $\begin{array}{l}42.2 \\
41.1 \\
39.6 \\
36.8 \\
35.9 \\
33.8\end{array}$ & $\begin{array}{l}43.6 \\
42.1 \\
38.6 \\
37.6 \\
36.2 \\
32.9\end{array}$ & $\begin{array}{l}41.5 \\
39.1 \\
36.1 \\
35.8 \\
27.3 \\
30.3\end{array}$ \\
\hline
\end{tabular}

Notes: Data supplied by Ministry of Justice (11 March 2009)

The Divorce Reform Act 1969 became operative on 1 January 1971; the Matrimonial and Family Proceedings Act came into effect on 12 October 1984. Figures include petitions for nullity 


\section{Notes to tables}

\section{Time series}

For most tables, years start at 1971 and then continue at five-year intervals until 1991. Individual years are shown thereafter.

\section{United Kingdom}

The United Kingdom comprises England, Wales, Scotland and Northern Ireland. The Channel Islands and the Isle of Man are not part of the United Kingdom.

\section{Population}

The estimated and projected resident population of an area includes all people who usually live there, whatever their nationality. Members of HM and US Armed Forces in the United Kingdom are included on a residential basis wherever possible. HM Forces stationed outside the United Kingdom are not included. Students are taken to be resident at their term time addresses.

Further information on population estimates is available on the National Statistics website at: www. statistics.gov.uk/popest

\section{Live births}

For England and Wales, figures relate to the number of births occurring in a period; for Scotland and Northern Ireland, figures relate to births registered in a period. By law, births must be registered within 42 days in England and Wales, within 21 days in Scotland, and within 42 days in Northern Ireland. In England and Wales, where a birth is registered later than the legal time period, and too late to be included in the count for the year of occurrence, it will be included in the count for the following year.

Age specific fertility rate (ASFR), is the number of births per 1,000 women in the stated age group.

The total fertility rate (TFR), which is the sum of the age-specific fertility rates expressed per woman, is a useful summary measure in that it can be used to examine both changes in fertility over time and between populations by removing the effect of different age distributions.

Care should be taken in interpreting the total fertility rate as indicative of future levels of childbearing. Used in this way it may be interpreted as the number of children that would be born to a woman if the current age-specific patterns of fertility persisted throughout her childbearing life. However, the TFR is a synthetic measure since age-specific fertility rates do not normally remain stable during the childbearing life span of a woman.

\section{Perinatal mortality}

In October 1992 the legal definition of a stillbirth was changed, from baby born dead after 28 completed weeks of gestation or more, to one born dead after 24 completed weeks of gestation or more.

\section{Period expectation of life}

The life tables on which these expectations are based use death rates for the given period to describe mortality levels for each year. Each individual year shown is based on a three-year period, so that for instance 1986 represents 1985-87. More details can be found at www.statistics.gov.uk/StatBase/Product. asp?vlnk=14459

\section{Deaths}

Figures for England and Wales relate to the number of deaths registered in each year up until 1992, and the number of deaths occurring in a year between 1993 and 2005 . From 2006 onwards, all figures relate to the number of deaths registered in the year. All figures for Scotland and Northern Ireland relate to the number of deaths registered in each year.

\section{Age-standardised mortality}

Directly age-standardised rates make allowances for changes in the age structure of the population. The age-standardised rate for a particular condition is that which would have occurred if the observed agespecific rates for the condition had applied in a given standard population. Table 2.2 uses the European Standard Population. This is a hypothetical population standard which is the same for both males and females allowing standardised rates to be compared for each sex, and between males and females.

\section{International Migration}

The UN recommends the following definition of an international long-term migrant:

An international long-term migrant is defined as a person who moves to a country other than that of his or her usual residence for a period of at least a year (12 months), so that the country of destination effectively becomes his or her new country of usual residence.

Figures in Tables 7.1-7.3 are compiled from several main sources of migration data:

- The richest source of information on international migrants comes from the International Passenger Survey (IPS), which is a sample survey of passengers arriving at, and departing from, the main United Kingdom air and sea ports and Channel Tunnel. This survey provides migration estimates based on respondents' intended length of stay in the UK or abroad and excludes most persons seeking asylum and some dependants of such asylum seekers.

- Two adjustments are made to account for people who do not realise their intended length of stay on arrival. First, visitor data from the IPS are used to estimate 'visitor switchers': those people who initially come to or leave the UK for a short period but subsequently stay for a year or longer. (For years before 2001, estimates of nonEuropean Economic Area (non-EEA) national visitor switcher inflows are made from the Home Office database of after-entry applications to remain in the UK). Second, people who intend to be migrants, but who in reality stay in the UK or abroad for less than a year ('migrant switchers'), are estimated from IPS migrant data.

- Home Office data on asylum seekers and their dependants.

- Estimates of migration between the UK and the Irish Republic estimated using information from the Irish Quarterly National Household Survey and the National Health Service Central Register, agreed between the Irish Central Statistics Office and the ONS

Estimates for 1999-2005 have been revised to take account of recent improvements in the methodology used to estimate migration. These improvements wer first published for 2006 data released in November 2007. More detail is provided in Appendix B of International Migration 2006 - MN Series no. 33

For years prior to 1991, the figures in Tables 7.1-7.3 are based only on data from the IPS. After taking into account of those groups of migrants known not to be covered by the IPS, it is estimated that the adjustmen needed to net migration ranges from about ten thousand in 1981 to just over twenty thousand in 1986 . From 1991, the figures in Tables 7.1-7.3 are based on data from all the sources and represent Total International Migration.

Old Commonwealth is defined as Australia, Canada, New Zealand and South Africa;

New Commonwealth is defined as all other Commonwealth countries.

Middle East is defined as Bahrain, Iran, Iraq, Israel, Jordan, Kuwait, Lebanon, Oman, Qatar, Saudi Arabia, Syria, the United Arab Emirates, and Yemen.

\section{Internal Migration}

Figures in Table 8.1 are based on the movement of NHS doctors' patients between former Health Authorities (HAs) in England and Wales, and Area Health Boards in Scotland and Northern Ireland. Yearly and quarterly figures have been adjusted to take account of differences in recorded cross-border flows between England and Wales, Scotland and Northern Ireland.

Prior to reorganisation of health authority databases from Family Health Service Authorities (FHSAs) to HAs some database boundaries were realigned. This included in a few cases transferring patients between databases to fit the new boundaries. For the most part, this movement was done outside the NHSCR system and therefore had no effect on migration data.
However a small number were transferred within the system. As migration estimates derived from NHSCR are the product of an administrative system (when patients re-register with GPs) this had the effect of generating small numbers of spurious migrants where no actual change of address had taken place. We have been advised of adjustments required to data by the Department of Health and these have been made to migration data.

The NHS Central Register (NHSCR) at Southport was computerised in early 1991, prior to which a three month time lag was assumed between a person moving and their re-registration with an NHS doctor being processed onto the NHSCR. Since computerisation, estimates of internal migration are based on the date of acceptance of the new patient by the HA (not previously available), and a one month time lag assumed.

It has been established that NHSCR data under-report the migration of males aged between 16 and 36 . Currently, however, there are no suitable sources of data available to enable adjustments or revisions to be made to the estimates. Further research is planned on this topic and new data sources may become available in the future. However, for the present time, historical estimates will not be revised and future estimates will not be adjusted.

\section{Marriages and divorces}

Marriages are tabulated according to date of solemnisation. Divorces are tabulated according to date of decree absolute. The term 'divorces' includes decrees of nullity. The fact that a marriage or divorce has taken place in England, Wales, Scotland or Northern Ireland does not mean either of the parties is resident there.

\section{Civil Partnerships}

The Civil Partnership Act 2004 came into force on 5 December 2005 in the UK, the first day couples could give notice of their intention to form a civil partnership. The first day that couples could formally form a partnership was 19 December 2005 in Northern Ireland, 20 December 2005 in Scotland and 21 December 2005 in England and Wales.

(18 civil partnerships were formed under special arrangements before these dates. These are included in the figures for England and Wales).

Civil partnerships are tabulated according to date of formation and area of occurrence. The fact that a civi partnership has taken place in England, Wales, Scotland or Northern Ireland does not necessarily mean either of the parties is resident there.

\section{EU Enlargement}

The coverage of European countries in Table 1.1 has been updated to reflect the enlargement of the EU to 27 member countries (EU27). On 1 May 2004, 10 new member countries were added: Cyprus, Czech Republic, Estonia, Hungary, Latvia, Lithuania, Malta, Poland, Slovakia and Slovenia. One 1 January 2007 a further 2 countries were added: Bulgaria and Romania.

\section{Sources}

Figures for Scotland and Northern Ireland have been provided by the General Register Office for Scotland and the Northern Ireland Statistics and Research Agency respectively. The International Passenger Survey (Tables 7.1-7.3) is conducted by the Surveys and Administrative Sources Directorate of ONS.

\section{Rounding}

All figures are rounded independently; constituent parts may not add to totals. Generally numbers and rates per 1,000 population are rounded to one decimal place (e.g 123.4); where appropriate, for small figures (below 10.0), two decimal places are given (e.g 7.62). Figures which are provisional or estimated are given in less detail (e.g 123 or 7.6 respectively) if their reliability does not justify giving the standard amount of detail. Where figures need to be treated with particular caution, an explanation is given as a footnote.

\section{Latest figures}

Figures for the latest quarters and years may be provisional and will be updated in future issues when later information becomes available. Where figures are not yet available, cells are left blank. 